\title{
Local-Global Compatibility and the Action of Monodromy on nearby Cycles
}

\section{Citation}

Caraiani, Ana. 2012. Local-Global Compatibility and the Action of Monodromy on nearby Cycles. Doctoral dissertation, Harvard University.

\section{Permanent link}

http://nrs.harvard.edu/urn-3:HUL.InstRepos:10086046

\section{Terms of Use}

This article was downloaded from Harvard University's DASH repository, and is made available under the terms and conditions applicable to Other Posted Material, as set forth at http:// nrs.harvard.edu/urn-3:HUL.InstRepos:dash.current.terms-of-use\#LAA

\section{Share Your Story}

The Harvard community has made this article openly available.

Please share how this access benefits you. Submit a story.

\section{Accessibility}


(C) 2012 - AnA CARAIANI

All Rights Reserved. 


\title{
LOCAL-GLOBAL COMPATIBILITY AND THE ACTION OF MONODROMY ON NEARBY CYCLES
}

\begin{abstract}
In this thesis, we study the compatibility between local and global Langlands correspondences for $G L_{n}$. This generalizes the compatibility between local and global class field theory and is related to deep conjectures in algebraic geometry and harmonic analysis, such as the Ramanujan-Petersson conjecture and the weight monodromy conjecture. Let $L$ be a CM field. We consider the case when $\Pi$ is a cuspidal automorphic representation of $G L_{n}\left(\mathbb{A}_{L}^{\infty}\right)$, which is conjugate self-dual and regular algebraic. Under these assumptions, there is an $l$-adic Galois representation $R_{l}(\Pi)$ associated to $\Pi$, which is known to be compatible with the local Langlands correspondence in most cases (for example, when $n$ is odd) and up to semisimplification in general. In this thesis, we complete the proof of the compatibility when $l \neq p$ by identifying the monodromy operator $N$ on both the local and the global sides. On the local side, the identification amounts to proving the Ramanujan-Petersson conjecture for $\Pi$ as above. On the global side it amounts to proving the weight-monodromy conjecture for part of the cohomology of a certain Shimura variety.
\end{abstract}




\section{Contents}

Abstract ........................ iii

Table of Contents . . . . . . . . . . . . . iv

Dedication ............................ vi

Acknowledgments . . . . . . . . . . . . . vii

1 Introduction 1

1.1 Langlands correspondences and local-global compatibility . . . . 1

1.2 History of the problem . . . . . . . . . . . . . . . . 4

1.3 The results and methods of this thesis $\ldots \ldots \ldots \ldots$

1.4 Organization of the thesis . . . . . . . . . . . . . 13

2 An integral model $\quad 15$

2.1 Shimura varieties . . . . . . . . . . . . . . . . . . . . 15

2.2 An integral model for Iwahori level structure $\ldots \ldots \ldots \ldots$

3 Sheaves of nearby cycles $\quad 39$

$3.1 \log$ structures . . . . . . . . . . . . . . . . . . 40

3.2 Nearby cycles and $\log$ schemes $\ldots \ldots \ldots$. . . . . . . . 42

4 The monodromy filtration $\quad 54$

4.1 Overview of the strictly semistable case . . . . . . . . . 54 
4.2 The product of strictly semistable schemes . . . . . . . . . 60

4.3 More general schemes . . . . . . . . . . . . . . . . . . . 82

5 The cohomology of closed strata $\quad 95$

5.1 Igusa varieties . . . . . . . . . . . . . . . . . . . . . . . . . 95

5.2 Counting points on Igusa varieties _ . . . . . . . . . . . 101

5.3 Vanishing of cohomology . . . . . . . . . . . . 106

6 The cohomology of Igusa varieties 119

7 Proof of the main theorem 142 
To Steven 


\section{Acknowledgments}

First of all, I would like to thank my advisor, Richard Taylor, for suggesting the topic of this dissertation and for his guidance, patience and generosity. This thesis owes a tremendous debt to his insights and expertise. I owe to him my mathematical development over the past five years and I am grateful for all that he has taught me.

I am also greatly indebted to many mathematicians for their willingness to share their insights and their knowledge with me, especially to Luc Illusie, Sophie Morel, Arthur Ogus, Sug Woo Shin and Teruyoshi Yoshida. I am grateful to Sophie Morel and Mark Kisin for reading this thesis and for their comments and also to the anonymous referees, who provided much useful feedback.

I also benefitted a great deal from interacting with my fellow graduate students at Harvard. I am grateful to them for useful conversations relevant to this thesis as well as for creating a stimulating number theoretic environment through numerous seminars and study groups. In particular, I am thankful to Thomas Barnet-Lamb, Carl Erickson, David Geraghty, Wushi Goldring, Katherine Korner, Bao Le Hung, Jay Pottharst, Sam Raskin, David Roe, Aaron Silberstein, David Smyth and Jack Thorne. I am also grateful to the staff of the Harvard Math Department, especially to Susan Gilbert, who made sure the administrative part of my graduate studies went smoothly. 
I am grateful to my closest friends for keeping me sane during graduate school. I thank Nikola Kamburov and Silviu Pufu for ski trips, movie nights and weekly brunch. I thank Tanya Khodorovskiy for teaching me many life skills, among which how to make borscht. I thank Alice Zhou for thoughtprovoking conversations over cookies and tea. I am also indebted to my fellow fifth-years at Harvard, who created an exceptionally friendly and supportive environment.

Finally, I would like to thank my family for all their love and support, especially my parents, Zoe and Cornel Caraiani, for always encouraging me to pursue my passions; and my husband, Steven Sivek, for making me laugh every day in the past seven years. 


\section{Chapter 1}

\section{Introduction}

\subsection{Langlands correspondences and local-global compatibility}

This thesis strengthens the compatibility of local and global Langlands correspondences for $G L_{n}$. The Langlands program provides a framework for generalizing class field theory, one of the major achievements of 20th century number theory, to the non-abelian setting. It is an intricate network of conjectures, meant to unify different areas of mathematics, such as representation theory, number theory and algebraic geometry. In the case of $G L_{n}$, the Langlands program predicts a global correspondence between automorphic representations of $G L_{n}$ and $n$-dimensional Galois representations, which we state below.

Conjecture 1.1.1. (Langlands, Fontaine-Mazur) Let $L$ be a number field and $l$ a rational prime. There is a bijection between the following sets consisting of 
isomorphism classes:

$$
\left\{\begin{array}{c}
\text { cuspidal automorphic } \\
\text { reps of } G L_{n}\left(\mathbb{A}_{L}\right) \\
\text { algebraic }
\end{array}\right\} \leftrightarrow\left\{\begin{array}{c}
\text { irred, cont. l-adic reps } \\
\text { Gal }(\bar{L} / L) \rightarrow G L_{n}\left(\overline{\mathbb{Q}}_{l}\right) \\
\text { unram. except at finitely many places } \\
\text { de Rham at l } l
\end{array}\right\} .
$$

The "cuspidal" condition on the automorphic side is expected to correspond to the "irreducible" condition on the Galois side. The "algebraic" condition is a condition on the infinitesimal character of the automorphic representation at $\infty$. This character can be thought of as a multiset of complex numbers via the Harish-Chandra isomorphism and the requirement is that these complex numbers be in fact integers. Its counterpart on the Galois side is the technical requirement that the Galois representation be "de Rham at l", which comes from $p$-adic Hodge theory. This conjecture is known in many special cases, but it is quite open in general. For $n=1$, the conjecture is a consequence of global class field theory.

At the same time, there is a local version of the Langlands correspondence, which in the case of $G L_{n}$ was constructed by Harris and Taylor in 2001 [HT]. This correspondence generalizes local class field theory. If we let $p$ be a rational prime and $K / \mathbb{Q}_{p}$ be a finite extension, then local class field theory gives a canonical isomorphism

$$
\operatorname{Art}_{K}: K^{\times} \rightarrow W_{K}^{\mathrm{ab}},
$$

which takes uniformizers to geometric Frobenius elements.

We now let $\operatorname{Irr}\left(G L_{n}(K)\right)$ denote the set of isomorphism classes of irreducible, smooth representations of $G L_{n}(K)$ over $\mathbb{C}$. Local Langlands is a correspondence between $\operatorname{Irr}\left(G L_{n}(K)\right)$ and certain objects very closely related to local Galois representations, called Weil-Deligne representations. A Weil-Deligne representation of the Weil group $W_{K}$ of $K$ over $\mathbb{C}$ is a triple $(V, r, N)$, where

- $V$ is a finite-dimensional $\mathbb{C}$-vector space 
- $r$ is a representation of $W_{K}$ on $V$ with open kernel

- and $N$ is a nilpotent endomorphism of $V$, which satisfies a certain compatibility with $r$, namely that for any $\sigma \in W_{K}$

$$
r(\sigma) N r(\sigma)^{-1}=\left|\operatorname{Art}_{K}^{-1}(\sigma)\right|_{K} N
$$

Weil-Deligne representations of $W_{K}$ are roughly equivalent to continuous representations of $\operatorname{Gal}(\bar{K} / K)$. A Weil-Deligne representation is called Frobenius semisimple if $r$ is semisimple. Let $\mathrm{WDRep}_{n}$ denote the set of isomorphism classes of $n$-dimensional Frobenius semisimple Weil-Deligne representations of $W_{K}$ over $\mathbb{C}$.

Theorem 1.1.2. (Harris-Taylor, Henniart) For any finite extension $K / \mathbb{Q}_{p}$, there exists a collection of bijections

$$
\operatorname{rec}_{K}: \operatorname{Irr}\left(G L_{n}(K)\right) \rightarrow \operatorname{WDRep}_{n}\left(W_{K}\right)
$$

for every $n \geq 1$ satisfying the following properties:

- for $n=1$ the bijection is via composition with $\mathrm{Art}_{K}^{-1}$.

- the bijection is compatible with twists by characters, with central characters and with duals.

- for $\left[\pi_{1}\right] \in \operatorname{Irr}\left(G L_{n_{1}}(K)\right)$ and $\left[\pi_{2}\right] \in \operatorname{Irr}\left(G L_{n_{2}}(K)\right)$ then the L-factors and $\epsilon$-factors of $\pi_{1} \times \pi_{2}$ and $\operatorname{rec}\left(\pi_{1}\right) \otimes \operatorname{rec}\left(\pi_{2}\right)$ are compatible.

The local and global correspondences are expected to be compatible, in the same way that local and global class field theory are compatible. For example, in the case of classsical modular forms, the compatibility ensures that the eigenvalues of Frobenius on the Galois side match up with the Hecke eigenvalues 
coming from the automorphic side. In general, the compatibility characterizes the global correspondence uniquely and its precise statement is as follows.

Conjecture 1.1.3. (Local-global compatibility) Keep the notations as in Conjecture 1.1.1 and Theorem 1.1.2. Fix an isomorphism $\iota_{l}: \overline{\mathbb{Q}}_{l} \simeq \mathbb{C}$. Let $\Pi$ be a cuspidal automorphic representation of $G L_{n}\left(\mathbb{A}_{L}\right)$ which is algebraic and let $R_{l}(\Pi)$ be the l-adic Galois representation associated to it.

Then for each place y of $L$ above a rational prime $p$ we have an isomorphism of Weil-Deligne representations

$$
W D\left(\left.R_{l}(\Pi)\right|_{G a l\left(\bar{L}_{y} / L_{y}\right)}\right)^{F-s s} \simeq \iota_{l}^{-1} \operatorname{rec}_{L_{y}}\left(\Pi_{y}^{\vee} \otimes|\operatorname{det}|^{\frac{1-n}{2}}\right) .
$$

\subsection{History of the problem}

The question of proving local-global compatibility has historically been interwtined with the question of constructing Galois representations. This is the traditional name for realizing the arrow

$$
\left\{\begin{array}{c}
\text { cuspidal automorphic } \\
\text { reps } \Pi \text { of } G L_{n}\left(\mathbb{A}_{L}\right) \\
\text { algebraic }
\end{array}\right\} \rightarrow\left\{\begin{array}{c}
\text { irred, cont. } l \text {-adic reps } \\
R_{l}(\Pi): \operatorname{Gal}(\bar{L} / L) \rightarrow G L_{n}\left(\mathbb{Q}_{l}\right) \\
\text { except at finitely many places } \\
\text { de Rham at } l
\end{array}\right\} .
$$

A general strategy is to find the Galois representation in the $l$-adic cohomology of certain algebraic varieties, called Shimura varieties. Other than global class field theory, the first major result in this direction goes back to Eichler-Shimura, Deligne and Deligne-Serre who constructed Galois representations associated to classical modular forms using modular curves.

For general $n$, a major breakthrough in the construction of Galois representations was obtained by Clozel [Cl1], who essentially constructed $R_{l}(\Pi)$ under the following conditions: 
- $L$ is a CM field,

- $\Pi$ satisfies $\Pi^{\vee} \simeq \Pi \circ c$, where $c$ is complex conjugation,

- $\Pi_{\infty}$ is regular algebraic,

- $\Pi$ is square-integrable at a finite place.

Roughly, the first two conditions ensure that $\Pi$ comes an automorphic representation of a unitary group via quadratic base change. The third condition ensures that this automorphic reprsentation for a unitary group can be "seen" in the cohomology of a unitary PEL-type Shimura variety. The fourth condition ensures that one can work with a unitary group with trivial endoscopy, which simplifies the trace formula used to compute the cohomology of the Shimura variety.

Under the same conditions on $\Pi$, Harris and Taylor [HT] proved local-global compatibility at all places of residual characteristic $p \neq l$ and up to semisimplification. This means that they obtained an isomorphism of $W_{K}$-representations, but did not pin down the monodromy operator $N$ coming from the global representation $R_{l}(\Pi)$. We comment briefly on the key geometric input of the argument. Harris and Taylor use a very special kind of Shimura variety, which, in addition to having trivial endoscopy, is associated to a unitary group with signature $(1, n-1) \times(0, n) \times \cdots \times(0, n)$ at infinity. It is a PEL-type Shimura variety, which means that it is a moduli space for abelian varieties, equipped with polarizations, endomorphisms and level structure. These abelian varieties have to satisfy an important compatibility, called the Kottwitz determinant condition [Ko1]. If one wants to compute Galois representations arising from cohomology of the Shimura variety, locally at a place $y$ of $L$, one can use an integral model defined over the ring of integers $\mathcal{O}_{L_{y}}$ of $L_{y}$. This integral model is proper, but in general not smooth. The moduli interpretation extends to the integral model, 
however, and the Kottwitz determinant condition determines the structure of the $p$-divisible group of each abelian variety ( $p$ here is the residue characteristic of $y)$. For signature $(1, n-1) \times(0, n) \times \ldots(0, n)$, the $p$-divisible group is essentially a one-dimensional Barsotti-Tate $\mathcal{O}_{L_{y}}$ - module. This leads to the discovery that, in some sense, the singularities of the integral model are the same as the singularities of the Lubin-Tate tower, whose cohomology realizes the local Langlands correspondence.

Taylor and Yoshida [TY] extended the compatibility under the four conditions above to Frobenius semisimplification. The idea for proving Conjecture 1.3.1 in this case is to show that both $W D\left(\left.R_{l}(\Pi)\right|_{G a l\left(\overline{L_{y}} / L_{y}\right.}\right)^{F-s s}$ and $\iota_{l}^{-1} \mathcal{L}_{n, L_{y}}\left(\Pi_{y}\right)$ have a remarkably elegant form, called "pure" in the terminology of $[\mathrm{TY}]$. A nice feature of purity is that it completely identifies the monodromy operator. Moreover, purity reflects certain deep intuitions coming from algebraic geometry and harmonic analysis.

To explain where the notion of purity originates, let $K$ be a $p$-adic field for $p \neq l$.

Definition 1.2.1. A Weil-Deligne representation $(V, r, N)$ of $W_{K}$ is called "strictly pure of weight $k$ " if the monodromy operator $N=0$ and if every eigenvalue of Frobenius is a Weil $q^{k}$-number, where $q$ is the cardinality of the residue field of $K$.

From the Weil conjectures [De1, De2] it follows that the etale cohomology of a proper, smooth variety over a finite field always gives rise to a strictly pure Weil-Deligne representation. However, the etale cohomology of a proper, smooth variety over $K$ doesn't have to be strictly pure, so a more general notion is needed in this case.

Definition 1.2.2. A Weil-Deligne representation $(V, r, N)$ is called "pure of weight $k$ " if $V$ has an increasing filtration $\mathrm{Fil}_{i}^{W}$, with $i \in k+\mathbb{Z}$ whose $i$ th graded 
piece is strictly pure of weight $i$ and such that $N^{i}$ induces an isomorphism $g r_{k+i}^{W} V \simeq g r_{k-i}^{W} V$.

The weight-monodromy conjecture ([De2, I2, RZ]) states that the etale cohomology of a proper, smooth variety over $K$ always gives rise to a pure Weil-Deligne representation. Therefore, identifying the monodromy operator in $W D\left(\left.R_{l}(\Pi)\right|_{\operatorname{Gal}\left(\overline{L_{y}} / L_{y}\right.}\right)^{F-s s}$ amounts to proving the weight-monodromy conjecture for part of the cohomology of a Shimura variety with signature $(1, n-1) \times$ $(0, n) \times \cdots \times(0, n)$.

On the other hand, the purity of $\iota_{l}^{-1} \mathcal{L}_{n, L_{y}}\left(\Pi_{y}\right)$ follows from the RamanujanPetersson conjecture for $\Pi$ as above. This conjecture predicts the fact that the local components at finite places of cuspidal automorphic representations of $G L_{n}\left(\mathbb{A}_{L}\right)$ (with unitary central characters) are tempered [Sar]. It is a generalization of the corresponding statement for Ramanujan's $\Delta$-function [Ra], which followed from Deligne's proof of the Weil conjectures [De1]. This conjecture, in the case where $\Pi$ is square-integrable at a finite place, had already been proved by Harris and Taylor [HT] as a consequence of the Weil conjectures, since they constructed $R_{l}(\Pi)$ in the cohomology of a proper, smooth variety over $L$.

The key insight of [TY] is that it is possible to prove the weight-monodromy conjecture for the Shimura variety with Iwahori level structure at $y$, in which case the cohomology of the generic fiber can be computed via the Rapoport-Zink weight spectral sequence. The inputs of the first page of the spectral sequence are the cohomologies of closed Newton polygon strata in the special fiber of the Shimura variety. These are proper, smooth schemes, whose cohomology realizes parts of the representation $\iota_{l}^{-1} \mathcal{L}_{n, L_{y}}\left(\Pi_{y}\right)^{s s}$. Using the machinery of [HT], Taylor and Yoshida compute the cohomology of each closed Newton polygon stratum explicitly and prove that the $\Pi^{\infty}$-part of each cohomology is concentrated in the middle dimension cohomology. Therefore, after restricting to the $\Pi^{\infty}$-part, the 
first page of the Rapoport-Zink weight spectral sequence is concentrated on a diagonal and so the spectral sequence degenerates at the first page. This provides exactly the filtration needed to prove the purity of $W D\left(\left.R_{l}(\Pi)\right|_{\text {Gal }\left(\overline{L_{y}} / L_{y}\right.}\right)^{F-s s}$.

Harris-Taylor and Taylor-Yoshida therefore proved Conjecture 1.3.1 under the assumption that $\Pi$ is square-integrable at a finite place. Shin [Sh3] later removed the condition that $\Pi$ be square-integrable at a finite place, by working with a unitary group with the same signature $(1, n-1) \times(0, n) \times \cdots \times(0, n)$, but allowing endoscopy. He constructed $R_{l}(\Pi)$ when $n$ is odd and established full local-global compatibility for $l \neq p$. The difficulty when $n$ is even comes from the fact that there is a cohomological obstruction to the existence of a unitary group with the desired signature at infinity and which is quasi-split. Shin found $R_{l}(\Pi)$ in the endoscopic part of the cohomology of a Shimura variety for $n+1$, but this is only "visible" when $\Pi_{\infty}$ satisfies an additional regularity condition. Under this condition, Shin again proved local-global compatibility in full for $l \neq p$. Chenevier and Harris $[\mathrm{CH}]$ then constructed the Galois representation in the missing cases through a $p$-adic deformation argument. However, their argument could only prove local-global compatibility for $l \neq p$ up to semisimplification.

In order to complete the proof of Conjecture 1.3.1 for $L$ a $\mathrm{CM}$ field and $\Pi$ a conjugate self-dual, regular algebraic, cuspidal automorphic representation of $G L_{n}\left(\mathbb{A}_{L}\right)$, one needs to treat the missing case of the compatibility: namely to identify the monodromy operator in the case when $n$ is even without any extra assumptions on $\Pi_{\infty}$. We remark that in this thesis we are concerned with localglobal compatibility at primes $p \neq l$. Simultaneously with writing this thesis, there has been a huge amount of progress in proving local-global compatibility when $p=l$ : the papers of Barnet-Lamb, Gee Geraghty and Taylor [BLGGT1, BLGGT2] prove the compatibility for $l=p$ under the same assumptions as $[\mathrm{Sh} 3, \mathrm{CH}]$. We have, since then, also found a way to identify the monodromy 
operator when $l=p$ and $n$ is even [Car], using different methods than those of this thesis.

\subsection{The results and methods of this thesis}

In this thesis we complete the proof of the following theorem.

Theorem 1.3.1. Let $n \in \mathbb{Z}_{\geq 2}$ be an integer and $L$ be any $C M$ field with complex conjugation c. Let l be a prime of $\mathbb{Q}$ and $\iota_{l}$ be an isomorphism $\iota_{l}: \overline{\mathbb{Q}}_{l} \rightarrow \mathbb{C}$. Let $\Pi$ be a cuspidal automorphic representation of $G L_{n}\left(\mathbb{A}_{L}\right)$ satisfying

- $\Pi^{\vee} \simeq \Pi \circ c$

- $\Pi$ is regular algebraic (this is the same as asking $\Pi$ to be cohomological for some irreducible algebraic representation $\Xi$ of $G L_{n}\left(L \otimes_{\mathbb{Q}} \mathbb{C}\right)$ ).

Let

$$
R_{l}(\Pi): \operatorname{Gal}(\bar{L} / L) \rightarrow G L_{n}\left(\overline{\mathbb{Q}}_{l}\right)
$$

be the Galois representation associated to $\Pi$ by [Sh3, CH]. Let $p \neq l$ and let $y$ be a place of $L$ above $p$. Then we have the following isomorphism of Weil-Deligne respresentations

$$
W D\left(\left.R_{l}(\Pi)\right|_{\operatorname{Gal}\left(\bar{L}_{y} / L_{y}\right)}\right)^{F-s s} \simeq \iota_{l}^{-1} \mathcal{L}_{n, L_{y}}\left(\Pi_{y}\right)
$$

Here $\mathcal{L}_{n, L_{y}}\left(\Pi_{y}\right)=\operatorname{rec}\left(\Pi_{y}^{\vee}\right) \otimes|\operatorname{det}|^{\frac{1-n}{2}}$ is the image of $\Pi_{y}$ under the local Langlands correspondence, where the geometric normalization is used.

In the process of proving Theorem 1.3.1, we also prove the RamanujanPetersson conjecture for $\Pi$ as above.

Theorem 1.3.2. Let $n \in \mathbb{Z}_{\geq 2}$ be an integer and $L$ be any $C M$ field. Let $\Pi$ be a cuspidal automorphic representation of $G L_{n}\left(\mathbb{A}_{L}\right)$ satisfying 
- $\Pi^{\vee} \simeq \Pi \circ c$

- $\Pi_{\infty}$ is cohomological for some irreducible algebraic representation $\Xi$ of $G L_{n}\left(L \otimes_{\mathbb{Q}} \mathbb{C}\right)$.

Then $\Pi$ is tempered at any finite place of $L$.

As mentioned in Section 1.2, the above theorems are already known when $n$ is odd or when $n$ is even and $\Pi$ is slightly regular, by work of Shin [Sh3]. They are also known if $\Pi$ is square integrable at a finite place, by the work of Harris-Taylor [HT] and Taylor-Yoshida [TY]. If $n$ is even then Chenevier and Harris construct in $[\mathrm{CH}]$ a global $\operatorname{Gal}(\bar{L} / L)$-representation $R_{l}(\Pi)$ which is compatible with the local Langlands correspondence up to semisimplification. Theorem 1.3.2 was proven by Clozel $[\mathrm{Cl} 2]$ at the places where $\Pi$ is unramified. We extend the local-global compatibility up to Frobenius semisimplification, by proving that both Weil-Deligne representations are pure.

The fact that $\iota_{l}^{-1} \mathcal{L}_{n, \mathcal{L}_{y}}\left(\Pi_{y}\right)$ is pure of some weight follows once we know Theorem 1.3.2 for all $\sigma \Pi$, where $\sigma \in \operatorname{Aut}(\mathbb{C})$. For the representation $W D\left(\left.R_{l}(\Pi)\right|_{\operatorname{Gal}\left(\bar{L}_{y} / L_{y}\right)}\right)$, our strategy is as follows: we find the Galois representation $R_{l}(\Pi)^{\otimes 2}$ in the cohomology of a system of Shimura varieties $X_{U}$ associated to a unitary group which looks like

$$
U(1, n-1) \times U(1, n-1) \times U(0, n)^{d-2}
$$

at infinity. Following the same structure of argument as Taylor-Yoshida in [TY], we prove that the Weil-Deligne representation associated to

$$
\left.R_{l}(\Pi)^{\otimes 2}\right|_{\operatorname{Gal}\left(\bar{L}_{y} / L\right)}
$$

is pure by explicitly computing the action of the monodromy operator $N$ on 
the cohomology of the system of Shimura varieties. We use Theorem 1.3.2 at a crucial point in the computation. We conclude that $W D\left(\left.R_{l}(\Pi)\right|_{\operatorname{Gal}\left(\bar{L}_{y} / L_{y}\right)}\right)^{F-s s}$ must also be pure of some weight.

To summarize, there are two main parts to the argument: a geometric input and a representation-theoretic input. The geometric input computes the action of the monodromy operator on the complex of nearby cycles on a certain kind of proper, but not smooth, scheme. This does not depend so much on the fact that the scheme we work with is a Shimura variety. The representation-theoretic input proves the Ramanujan-Petersson conjecture via a stable trace formula for computing the cohomology of Shimura varieties or other closely related varieties.

We start by explaining the geometric input, i.e. we briefly outline our computation of the action of $N$ on the Weil-Deligne representation associated to $\left.R_{l}(\Pi)^{\otimes 2}\right|_{\operatorname{Gal}_{\left(\bar{L}_{y} / L_{y}\right)}}$. First, we base change $\Pi$ to a CM field $F^{\prime}$ such that there is a place $\mathfrak{p}$ of $F^{\prime}$ above the place $y$ of $L$ where $B C_{F^{\prime} / L}(\Pi)_{\mathfrak{p}}$ has an Iwahori fixed vector. It suffices to study the Weil-Deligne representation corresponding to $\Pi^{0}=B C_{F^{\prime} / L}(\Pi)$ and prove that it is pure. We then take a quadratic extension $F$ of $F^{\prime}$ which is also a CM field and in which the place $\mathfrak{p}$ splits $\mathfrak{p}=\mathfrak{p}_{1} \mathfrak{p}_{2}$. We let $\sigma \in \operatorname{Gal}\left(F / F^{\prime}\right)$ be the automorphism which sends $\mathfrak{p}_{1}$ to $\mathfrak{p}_{2}$. We choose $F$ and $F^{\prime}$ such that they contain an imaginary quadratic field $E$ in which $p$ splits. We take a $\mathbb{Q}$-group $G$ which satisfies the following:

- $G$ is quasi-split at all finite places.

- $G(\mathbb{R})$ has signature $(1, n-1)$ at two embeddings which differ by $\sigma$ and $(0, n)$ everywhere else.

- $G\left(\mathbb{A}_{E}\right) \simeq G L_{1}\left(\mathbb{A}_{E}\right) \times G L_{n}\left(\mathbb{A}_{F}\right)$.

We let $\Pi^{1}=B C_{F / F^{\prime}}\left(\Pi^{0}\right)$. Then the Galois representation $R_{l}\left(\Pi^{0}\right)$ can be seen in the $\Pi^{1, \infty}$-part of the (base change of the) cohomology of a system of Shimura 
varieties associated to $G$. We let $X_{U}$ be the inverse system of Shimura varieties associated to the group $G$. We let the level $U$ vary outside $\mathfrak{p}_{1} \mathfrak{p}_{2}$ and be equal to the Iwahori subgroup at $\mathfrak{p}_{1}$ and $\mathfrak{p}_{2}$. We construct an integral model of $X_{U}$ which parametrizes abelian varieties with Iwahori level structure at $\mathfrak{p}_{1}$ and $\mathfrak{p}_{2}$. By abuse of notation, we will denote this integral model by $X_{U}$ as well. The special fiber $Y_{U}$ of $X_{U}$ has a stratification by $Y_{U, S, T}$ where the $S, T \subseteq\{1, \ldots n\}$ are related to the Newton polygons of the $p$-divisible groups above $\mathfrak{p}_{1}$ and $\mathfrak{p}_{2}$. We compute the completed strict local rings at closed geometric points of $X_{U}$ and use this computation to show that $X_{U}$ is locally etale over a product of strictly semistable schemes, which on the special fiber are closely related to the strata $Y_{U, S, T}$. If we let $\mathcal{A}_{U}$ be the universal abelian variety over $X_{U}$, then $\mathcal{A}_{U}$ has the same stratification and the same geometry as $X_{U}$.

Let $\xi$ be an irreducible algebraic representation of $G$ over $\overline{\mathbb{Q}}_{l}$, which determines non-negative integers $t_{\xi}, m_{\xi}$ and an endomorphism $a_{\xi} \in \operatorname{End}\left(\mathcal{A}_{U}^{m_{\xi}} / X_{U}\right) \otimes_{\mathbb{Z}}$ $\mathbb{Q}$. We are interested in understanding the $\Pi^{1, \infty}$-part of

$$
H^{j}\left(X_{U}, \mathcal{L}_{\xi}\right)=a_{\xi} H^{j+m_{\xi}}\left(\mathcal{A}_{U}^{m_{\xi}}, \overline{\mathbb{Q}}_{l}\left(t_{\xi}\right)\right)
$$

Thus, we study the cohomology of the generic fiber $H^{j}\left(\mathcal{A}_{U}^{m_{\xi}}, \overline{\mathbb{Q}}_{l}\right)$ and we do so via the cohomology of the complex of nearby cycles $R \psi \overline{\mathbb{Q}}_{l}$ over the special fiber of $\mathcal{A}_{U}^{m_{\xi}}$. The key ingredients in studying the complex of nearby cycles together with the action of monodromy are logarithmic schemes, the weight spectral sequence as constructed by Saito [Sa2] (which on the level of complexes of sheaves describes the action of monodromy on the complex of nearby cycles for strictly semistable schemes), and the formula

$$
\left(R \psi \overline{\mathbb{Q}}_{l}\right)_{X_{1} \times X_{2}} \simeq\left(R \psi \overline{\mathbb{Q}}_{l}\right)_{X_{1}} \otimes^{L}\left(R \psi \overline{\mathbb{Q}}_{l}\right)_{X_{2}}
$$


when $X_{1}$ and $X_{2}$ are semistable schemes. Using these ingredients, we deduce the existence a spectral sequence relating terms of the form $H^{j}\left(\mathcal{A}_{U, S, T}^{m_{\xi}}, \overline{\mathbb{Q}}_{l}\right)$ (up to twisting and shifting) to the object we're interested in, $H^{j}\left(\mathcal{A}_{U}^{m_{\xi}}, \overline{\mathbb{Q}}_{l}\right)$. This spectral sequence is, in some sense, a generalization of the Rapoport-Zink spectral sequence [RZ] for a strictly semistable scheme to the case of a scheme which looks locally like a product of strictly semistable schemes.

At this stage, the representation-theoretic input comes into play. The cohomology of each stratum $H^{j}\left(\mathcal{A}_{U, S, T}^{m_{\xi}}, \overline{\mathbb{Q}}_{l}\right)$ is closely related to the cohomology of Igusa varieties. The next step is to compute the $\Pi^{1, \infty}$-part of the cohomology of certain Igusa varieties, for which we adapt the strategy of Theorem 6.1 of [Sh3] and the stable trace formula deduced in [Sh2]. Using the result on Igusa varieties, we prove Theorem 1.3.2 and then we also make use of the classification of tempered representations. We prove that the $\Pi^{1, \infty}$-part of each $H^{j}\left(\mathcal{A}_{U, S, T}^{m_{\xi}}, \overline{\mathbb{Q}}_{l}\right)$ vanishes outside the middle dimension and thus that our spectral sequence degenerates at $E_{1}$. The $E_{1}$ page of the spectral sequence provides us with the exact filtration of the $\Pi^{1, \infty}$-part of

$$
\lim _{\overrightarrow{U^{p}}} H^{2 n-2}\left(X_{U}, \mathcal{L}_{\xi}\right)
$$

which exhibits its purity.

\subsection{Organization of the thesis}

We now describe the organization of this thesis. In Chapter 2 we define the PEL datum, unitary group and Shimura varieties we shall work with, we define integral models for these Shimura varieties as well as stratifications of their special fibers. We study the geometry of the integral model with Iwahori level structure, both globally, in terms of the stratification, and locally, in which case 
it essentially looks like a product of strictly semistable schemes.

In Chapter 3 we define a log structure over the Shimura variety with Iwahori level structure and show that this log structure gives rise to a log smooth scheme. We review and use Nakayama's results on nearby cycles for log smooth schemes $[\mathrm{Na}]$, computing the sheaves of nearby cycles on our Shimura variety explicitly. We also review Illusie's computation of the complex of nearby cycles in the case of a product of strictly semistable schemes [I2].

In Chapter 4 we review Saito's construction of the weight spectral sequence for a strictly semistable schemes [Sa2], but motivate it using log schemes and $\log$ structures. We then use the computations in Chapter 3 to identify the monodromy filtration on the complex of nearby cycles, first in the case of a product of strictly semistable schemes, then in the case matching our Iwahorilevel Shimura variety. This makes use of the framework of perverse sheaves. We obtain our spectral sequence relating the cohomologies of the closed Newton polygon strata to the cohomology of the Iwahori-level Shimura variety.

In Chapter 5 we relate the closed Newton polygon strata to Igusa varieties, we prove Theorem 1.3.2 (the Ramanujan-Petersson conjecture) and we show that the $\Pi^{\infty}$-part of the cohomology of the closed Newton polygon strata vanishes outside the middle dimension. The results of Chapter 5 depend on a cohomological computation for Igusa varieties, which we defer until the next chapter.

In Chapter 6 we use Shin's stable trace formula for Igusa varieties [Sh2] to compute the cohomology of the Igusa varieties we're working with. This section follows [Sh3] very closely and makes use of the trace formula, endoscopy (which we show plays a trivial part in our case) and local base change.

In Chapter 7 we put the results of the previous chapters together and prove the main theorem, Theorem 1.3.1. 


\section{Chapter 2}

\section{An integral model}

\subsection{Shimura varieties}

Let $E$ be an imaginary quadratic field in which $p$ splits, let $c$ be the non-trivial element in $\operatorname{Gal}(E / \mathbb{Q})$ and choose a prime $u$ of $E$ above $p$. From now on, we assume that $n$ is an even positive integer.

Let $F_{1}$ be a totally real field of finite degree over $\mathbb{Q}$ and $w$ a prime of $F_{1}$ above $p$. Let $F_{2}$ be a quadratic totally real extension of $F_{1}$ in which $w$ splits $w=w_{1} w_{2}$. Let $d=\left[F_{2}: \mathbb{Q}\right]$ and we assume that $d \geq 3$. Let $F=F_{2}$.E. Let $\mathfrak{p}_{i}$ be the prime of $F$ above $w_{i}$ and $u$ for $i=1,2$. We denote by $\mathfrak{p}_{i}$ for $2<i \leq r$ the rest of the primes which lie above the prime $u$ of $E$. We choose embeddings $\tau_{i}: F \hookrightarrow \mathbb{C}$ with $i=1,2$ such that $\tau_{2}=\tau_{1} \circ \sigma$, where $\sigma$ is the element of $\operatorname{Gal}(F / \mathbb{Q})$ which takes $\mathfrak{p}_{1}$ to $\mathfrak{p}_{2}$. In particular, this means that $\tau_{E}:=\left.\tau_{1}\right|_{E}=\left.\tau_{2}\right|_{E}$ is well-defined. By abuse of notation we will also denote by $\sigma$ the Galois automorphism of $F_{2}$ taking $w_{1}$ to $w_{2}$.

We will work with a Shimura variety corresponding to the PEL datum $(F, *, V,\langle\cdot, \cdot\rangle, h)$, where $F$ is the CM field defined above and $*=c$ is the in- 
volution given by complex conjugation. We take $V$ to be the $F$-vector space $F^{n}$ for some integer $n$. The pairing

$$
\langle\cdot, \cdot\rangle: V \times V \rightarrow \mathbb{Q}
$$

is a non-degenerate Hermitian pairing such that $\left\langle f v_{1}, v_{2}\right\rangle=\left\langle v_{1}, f^{*} v_{2}\right\rangle$ for all $f \in F$ and $v_{1}, v_{2} \in V$. The last element we need is an $\mathbb{R}$-algebra homomorphism $h: \mathbb{C} \rightarrow \operatorname{End}_{F}(V) \otimes_{\mathbb{Q}} \mathbb{R}$ such that the bilinear pairing

$$
\left(v_{1}, v_{2}\right) \rightarrow\left\langle v_{1}, h(i) v_{2}\right\rangle
$$

is symmetric and positive definite.

We define an algebraic group $G$ over $\mathbb{Q}$ by

$$
G(R)=\left\{(g, \lambda) \in \operatorname{End}_{F \otimes_{\mathbb{Q}} R}\left(V \otimes_{\mathbb{Q}} R\right) \times R^{\times} \mid\left\langle g v_{1}, g v_{2}\right\rangle=\lambda\left\langle v_{1}, v_{2}\right\rangle\right\}
$$

for any $\mathbb{Q}$-algebra $R$. For $\sigma \in \operatorname{Hom}_{E, \tau_{E}}(F, \mathbb{C})$ we let $\left(p_{\sigma}, q_{\sigma}\right)$ be the signature at $\sigma$ of the pairing $\langle\cdot, \cdot\rangle$ on $V \otimes_{\mathbb{Q}} \mathbb{R}$. We claim that we can find a PEL datum as above, such that $\left(p_{\tau}, q_{\tau}\right)=(1, n-1)$ for $\tau=\tau_{1}$ or $\tau_{2}$ and $\left(p_{\tau}, q_{\tau}\right)=(0, n)$ otherwise and such that $G_{\mathbb{Q}_{v}}$ is quasi-split at every finite place $v$.

Lemma 2.1.1. Let $F$ be a $C M$ field as above. For any embeddings $\tau_{1}, \tau_{2}: F \hookrightarrow$ $\mathbb{C}$ there exists a PEL datum $(F, *, V,\langle\cdot, \cdot\rangle, h)$ as above such that the associated group $G$ is quasi-split at every finite place and has signature $(1, n-1)$ at $\tau_{1}$ and $\tau_{2}$ and $(0, n)$ everywhere else.

Proof. This lemma is standard and follows from computations in Galois cohomology found in section 2 of [Cl1], but see also Lemma 1.7 of [HT]. The problem is that of constructing a global unitary similitude group with prescribed local conditions. It is enough to consider the case of a unitary group $G^{0}$ over $\mathbb{Q}$, 
by taking it to be the algebraic group defined by $\operatorname{ker}\left(G(R) \rightarrow R^{\times}\right)$sending $(g, \lambda) \mapsto \lambda$.

A group $G$ defined as above has a quasi-split inner form over $\mathbb{Q}$ denoted $G_{n}$, defined as in section 3 of [Sh3]. This inner form $G_{n}$ is the group of similitudes which preserve the non-degenerate Hermitian pairing $\left\langle v_{1}, v_{2}\right\rangle=v_{1} \zeta \Phi^{t} v_{2}^{c}$ with $\Phi \in G L_{n}(\mathbb{Q})$ having entries

$$
\Phi_{i j}=(-1)^{i+1} \delta_{i, n+1-j}
$$

and $\zeta \in F^{*}$ an element of trace 0 . Let $G^{\prime}$ be the adjoint group of $G_{n}^{0}$. It suffices to show that the tuple of prescribed local conditions, classified by elements in $\oplus_{v} H^{1}\left(F_{2, v}, G^{\prime}\right)$, is in the image of the map

$$
H^{1}\left(F_{2}, G^{\prime}\right) \rightarrow \oplus_{v} H^{1}\left(F_{2, v}, G^{\prime}\right)
$$

where the sum is taken over all places $v$ of $F_{2}$. For $n$ odd, Lemma 2.1 of [Cl1] ensures that the above map is surjective, so there is no cohomological obstruction for finding the global unitary group. In the case we are interested in, $n$ is even and the image of the above map is equal to the kernel of

$$
\bigoplus_{v} H^{1}\left(F_{2, v}, G^{\prime}\right) \rightarrow \mathbb{Z} / 2 \mathbb{Z}
$$

We can use Lemma 2.2 of [Cl1] to compute all the local invariants (i.e. the images of $H^{1}\left(F_{2, v}, G^{\prime}\right) \rightarrow \mathbb{Z} / 2 \mathbb{Z}$ for all places $\left.v\right)$. At the finite places, the sum of the invariants is $0(\bmod 2)$ (this is guaranteed by the existence of the quasi-split inner form $G_{n}$ of $G$, which has the same local invariants at finite places). At the infinite places $\tau_{1}$ and $\tau_{2}$ the invariants are $\frac{n}{2}+1(\bmod 2)$ and at all other infinite places they are $\frac{n}{2}(\bmod 2)$. The global invariant is $\frac{n d}{2}+2(\bmod 2)$, 
where $d$ is the degree of $F_{2}$ over $\mathbb{Q}$. Since $d$ is even, the image in $\mathbb{Z} / 2 \mathbb{Z}$ is equal to $0(\bmod 2)$, so the prescribed local unitary groups arise from a global unitary group.

We will choose the $\mathbb{R}$-homomorphism $h: \mathbb{C} \rightarrow \operatorname{End}_{F}(V) \otimes_{\mathbb{Q}} \mathbb{R}$ such that under the natural $\mathbb{R}$-algebra isomorphism $\operatorname{End}_{F}(V)_{\mathbb{R}} \simeq \prod_{\left.\tau\right|_{E}=\tau_{E}} M_{n}(\mathbb{C})$ it equals

$$
z \mapsto\left(\left(\begin{array}{cc}
z I_{p_{\tau}} & 0 \\
0 & \bar{z} I_{q_{\tau}}
\end{array}\right)_{\tau}\right)
$$

where $\tau$ runs over elements of $\operatorname{Hom}_{E, \tau_{E}}(F, \mathbb{C})$.

Now that we've defined the PEL datum we can set up our moduli problem. Note that the reflex field of the PEL datum is $F^{\prime}=F_{1} \cdot E$. Let $S / F^{\prime}$ be a scheme and $A / S$ an abelian scheme of dimension $d n$. Suppose we have an embedding $i: F \hookrightarrow \operatorname{End}(A) \otimes_{\mathbb{Z}} \mathbb{Q}$. Lie $A$ is a locally free $\mathcal{O}_{S}$-module of rank $d n$ with an action of $F$. We can decompose $\operatorname{Lie} A=\operatorname{Lie}^{+} A \oplus \mathrm{Lie}^{-} A$ where $\mathrm{Lie}^{+} A=\operatorname{Lie} A \otimes_{\mathcal{O}_{S} \otimes E} \mathcal{O}_{\mathcal{S}}$ and the map $E \hookrightarrow F^{\prime} \rightarrow \mathcal{O}_{\mathcal{S}}$ is the natural map followed by the structure map. $\operatorname{Lie}^{-} A$ is defined in the same way using the complex conjugate of the natural map $E \hookrightarrow F^{\prime}$. We ask that $\mathrm{Lie}^{+} A$ be a free $\mathcal{O}_{S}$-module of rank 2 and that $\operatorname{Lie}^{+} A \simeq \mathcal{O}_{S} \otimes_{F_{1}} F_{2}$ as an $\mathcal{O}_{S}$-module with an action of $F_{2}$.

Definition 2.1.2. If the the conditions above are satisfied, we will call the pair $(A, i)$ compatible.

Remark. This is an adaptation to our situation of the notion of compatibility defined in section III.1 of [HT], which fulfills the same purpose as the determinant condition defined on page 390 of [Ko1].

For an open compact subgroup $U \subset G\left(\mathbb{A}^{\infty}\right)$ we consider the contravariant 
functor $\mathfrak{X}_{U}$ mapping

$$
\begin{aligned}
& \left(\begin{array}{c}
\text { Connected, locally noetherian } \\
F^{\prime} \text {-schemes with geometric point } \\
(S, s)
\end{array}\right) \rightarrow(\text { Sets }) \\
& (S, s) \mapsto\{(A, \lambda, i, \bar{\eta})\} / \sim
\end{aligned}
$$

where

- $A$ is an abelian scheme over $S$;

- $\lambda: A \rightarrow A^{\vee}$ is a polarization;

- $i: F \hookrightarrow \operatorname{End}^{0}(A)=\operatorname{End} A \otimes_{\mathbb{Z}} \mathbb{Q}$ is such that $(A, i)$ is compatible and $\lambda \circ i(f)=i\left(f^{*}\right)^{\vee} \circ \lambda$, for all $f \in F$;

- $\bar{\eta}$ is a $\pi_{1}(S, s)$-invariant $U$-orbit of isomorphisms of Hermitian $F \otimes_{\mathbb{Q}} \mathbb{A}^{\infty}$ modules

$$
\eta: V \otimes_{\mathbb{Q}} \mathbb{A}^{\infty} \rightarrow V A_{s}
$$

which take the fixed pairing $\langle\cdot, \cdot\rangle$ on $V$ to on $\left(\mathbb{A}^{\infty}\right)^{\times}$-multiple of the $\lambda$-Weil pairing on $V A_{s}$. Here,

$$
V A_{s}=\left(\lim _{\leftarrow} A[N](k(s))\right) \otimes_{\mathbb{Z}} \mathbb{Q}
$$

is the adelic Tate module.

We consider two quadruples as above equivalent if there is an isogeny between the abelian varieties which is compatible with the additional structures. If $s^{\prime}$ is a different geometric point of $S$ then there is a canonical bijection between $\mathfrak{X}_{U}(S, s)$ and $\mathfrak{X}_{U}\left(S, s^{\prime}\right)$. We can forget about the geometric points and extend the definition from connected to arbitrary locally noetherian $F^{\prime}$-schemes. When 
$U$ is sufficiently small, this functor is representable by a smooth and quasiprojective variety $X_{U} / F^{\prime}$ of dimension $2 n-2$ (this is explained on page 391 of $\left[\right.$ Ko1]). The variety $X_{U}$ is a disjoint union of $\left|\operatorname{ker}^{1}(G, \mathbb{Q})\right|$ copies of the canonical model of the Shimura variety. As $U$ varies, the inverse system of the $X_{U}$ has a natural right action of $G\left(\mathbb{A}^{\infty}\right)$.

Let $\mathcal{A}_{U}$ be the universal abelian variety over $X_{U}$. The action of $G\left(\mathbb{A}^{\infty}\right)$ on the inverse system of the $X_{U}$ extends to an action by quasi-isogenies on the inverse system of the $\mathcal{A}_{U}$. The following construction goes through as in section III. 2 of [HT]. Let $l$ be a rational prime (we impose no conditions on $l$ yet, but we will restrict to $l$ different from $p$ when we work with an integral model over the ring of integers in a $p$-adic field) and let $\xi$ an irreducible algebraic representation of $G$ over $\mathbb{Q}_{l}^{a c}$. This defines a lisse $\mathbb{Q}_{l}^{a c}$-sheaf $\mathcal{L}_{\xi, l}$ over each $X_{U}$ and the action of $G\left(\mathbb{A}^{\infty}\right)$ extends to the inverse system of sheaves. The direct limit

$$
H^{i}\left(X, \mathcal{L}_{\xi, l}\right)=\lim _{\rightarrow} H^{i}\left(X_{U} \times_{F^{\prime}} \bar{F}^{\prime}, \mathcal{L}_{\xi, l}\right)
$$

is a (semisimple) admissible representation of $G\left(\mathbb{A}^{\infty}\right)$ with a continuous action of $\operatorname{Gal}\left(\bar{F}^{\prime} / F^{\prime}\right)$. We can decompose it as

$$
H^{i}\left(X, \mathcal{L}_{\xi, l}\right)=\bigoplus_{\pi} \pi \otimes R_{\xi, l}^{i}(\pi)
$$

where the sum runs over irreducible admissible representations $\pi$ of $G\left(\mathbb{A}^{\infty}\right)$ over $\mathbb{Q}_{l}^{a c}$. The $R_{\xi, l}^{i}(\pi)$ are finite dimensional continuous representations of $\operatorname{Gal}\left(\bar{F}^{\prime} / F^{\prime}\right)$ over $\mathbb{Q}_{l}^{a c}$. We shall suppress the $l$ from $\mathcal{L}_{\xi . l}$ and $R_{\xi, l}^{i}(\pi)$ where it is understood from context. To the irreducible representation $\xi$ of $G$ we can associate as in section III.2 of [HT] non-negative integers $m_{\xi}$ and $t_{\xi}$ and an idempotent $\epsilon_{\xi} \in \mathbb{Q}\left[S_{m_{\xi}}\right]$ (where $S_{m_{\xi}}$ is the symmetric group on $m_{\xi}$ letters). As 
on page 476 of [TY], define for each integer $N \geq 2$,

$$
\epsilon\left(m_{\xi}, N\right)=\prod_{x=1}^{m_{\xi}} \prod_{y \neq 1} \frac{[N]_{x}-N}{N-N^{y}} \in \mathbb{Q}\left[\left(N^{\mathbb{Z} \geq 0}\right)^{m_{\xi}}\right],
$$

where $[N]_{x}$ denotes the endomorphism generated by multiplication by $N$ on the $x$-th factor and $y$ ranges from 0 to $2\left[F_{2}: \mathbb{Q}\right] n^{2}$ but excluding 1 . Set

$$
a_{\xi}=a_{\xi, N}=\epsilon_{\xi} P\left(\epsilon\left(m_{\xi}, N\right)\right)
$$

which can be thought of as an element of $\operatorname{End}\left(\mathcal{A}_{U}^{m_{\xi}} / X_{U}\right) \otimes_{\mathbb{Z}} \mathbb{Q}$. Here $P\left(\epsilon\left(m_{\xi}, N\right)\right)$ is the polynomial

$$
P(X)=\left((X-1)^{4 n-3}+1\right)^{4 n-3} .
$$

If we let proj : $\mathcal{A}_{U}^{m_{\xi}} \rightarrow X_{U}$ be the natural projection, then $\epsilon\left(m_{\xi}, N\right)$ is an idempotent on each of the sheaves $R^{j} \operatorname{proj}_{*} \overline{\mathbb{Q}}_{l}\left(t_{\xi}\right)$, hence also on

$$
H^{i}\left(X_{U} \times_{F^{\prime}} \bar{F}^{\prime}, R^{j} \operatorname{proj}_{*} \overline{\mathbb{Q}}_{l}\left(t_{\xi}\right)\right) \Rightarrow H^{i+j}\left(\mathcal{A}_{U}^{m_{\xi}} \times_{F^{\prime}} \bar{F}^{\prime}, \overline{\mathbb{Q}}_{l}\left(t_{\xi}\right)\right) .
$$

We get an endomorphism $\epsilon\left(m_{\xi}, N\right)$ of $H^{i+j}\left(\mathcal{A}_{U}^{m_{\xi}} \times_{F^{\prime}} \bar{F}^{\prime}, \overline{\mathbb{Q}}_{l}\left(t_{\xi}\right)\right)$ which is an idempotent on each graded piece of a filtration of length at most $4 n-3$. In this case, $P\left(\epsilon\left(m_{\xi}, N\right)\right)$ must be an idempotent on all of $H^{i+j}\left(\mathcal{A}_{U}^{m_{\xi}} \times_{F^{\prime}} \bar{F}^{\prime}, \overline{\mathbb{Q}}_{l}\left(t_{\xi}\right)\right)$. We have an isomorphism

$$
H^{i}\left(X_{U} \times_{F^{\prime}} \bar{F}^{\prime}, \mathcal{L}_{\xi}\right) \cong a_{\xi} H^{i+m_{\xi}}\left(\mathcal{A}_{U}^{m_{\xi}} \times_{F^{\prime}} \bar{F}^{\prime}, \overline{\mathbb{Q}}_{l}\left(t_{\xi}\right)\right),
$$

which commutes with the action of $G\left(\mathbb{A}^{\infty}\right)$. 


\subsection{An integral model for Iwahori level structure}

Let $K=F_{\mathfrak{p}_{1}} \simeq F_{\mathfrak{p}_{2}}$, where the isomorphism is via $\sigma$, denote by $\mathcal{O}_{K}$ the ring of integers of $K$ and by $\pi$ a uniformizer of $\mathcal{O}_{K}$.

Let $S / \mathcal{O}_{K}$ be a scheme and $A / S$ an abelian scheme of dimension $d n$. Suppose we have an embedding $i: \mathcal{O}_{F} \hookrightarrow \operatorname{End}(A) \otimes_{\mathbb{Z}} \mathbb{Z}_{(p)}$. Lie $A$ is a locally free $\mathcal{O}_{S^{-}}$ module of rank $d n$ with an action of $F$. We can decompose $\operatorname{Lie} A=\operatorname{Lie}^{+} A \oplus$ $\mathrm{Lie}^{-} A$ where $\operatorname{Lie}^{+} A=\operatorname{Lie} A \otimes_{\mathbb{Z}_{p} \otimes \mathcal{O}_{E}} \mathcal{O}_{E, u}$. There are two natural actions of $\mathcal{O}_{F}$

on $\mathrm{Lie}^{+} A$, via $\mathcal{O}_{F} \rightarrow \mathcal{O}_{F_{\mathfrak{p}_{j}}} \stackrel{\sim}{\rightarrow} \mathcal{O}_{K}$ composed with the structure map for $j=1,2$. These two actions differ by the automorphism $\sigma \in \operatorname{Gal}(F / \mathbb{Q})$. There is also a third action via the embedding $i$ of $\mathcal{O}_{F}$ into the ring of endomorphisms of $A$. We ask that $\operatorname{Lie}^{+} A$ be locally free of rank 2 , that the part of $\mathrm{Lie}^{+} A$ where the first action of $\mathcal{O}_{F}$ on $\mathrm{Lie}^{+} A$ coincides with $i$ be locally free of rank 1 and that the part where the second action coincides with $i$ also be locally free of rank 1 .

Definition 2.2.1. If the above conditions are satisfied, then we call $(A, i) \mathrm{com}$ patible. One can check that for $S / K$ this notion of compatibility coincides with the one in Definition 2.1.2.

If $p$ is locally nilpotent on $S$ then $(A, i)$ is compatible if and only if

- $A\left[\mathfrak{p}_{i}^{\infty}\right]$ is a compatible, one-dimensional Barsotti-Tate $\mathcal{O}_{K}$-module for $i=$ 1,2 and

- $A\left[\mathfrak{p}_{i}^{\infty}\right]$ is ind-etale for $i>2$.

By a compatible Barsotti-Tate $\mathcal{O}_{K}$-module we mean that the two actions on it by $\mathcal{O}_{K}$, via endomorphisms or via the structure map coincide.

We will now define a few integral models for our Shimura varieties $X_{U}$. We can decompose $G\left(\mathbb{A}^{\infty}\right)$ as

$$
G\left(\mathbb{A}^{\infty}\right)=G\left(\mathbb{A}^{\infty, p}\right) \times \mathbb{Q}_{p}^{\times} \times \prod_{i=1}^{r} G L_{n}\left(F_{\mathfrak{p}_{i}}\right) .
$$


For each $i$, let $\Lambda_{i}$ be an $\mathcal{O}_{F_{\mathfrak{p}_{i}}}$-lattice in $F_{\mathfrak{p}_{i}}^{n}$ which is stable under $G L_{n}\left(\mathcal{O}_{F_{\mathfrak{p}_{i}}}\right)$ and self-dual with respect to $\langle\cdot, \cdot\rangle$. For each $\vec{m}=\left(m_{1}, \ldots, m_{r}\right)$ and compact open $U^{p} \subset G\left(\mathbb{A}^{\infty, p}\right)$ we define the compact open subgroup $U^{p}(\vec{m})$ of $G\left(\mathbb{A}^{\infty}\right)$ as

$$
U^{p}(\vec{m})=U^{p} \times \mathbb{Z}_{p}^{\times} \times \prod_{i=1}^{r} \operatorname{ker}\left(G L_{\mathcal{O}_{F_{\mathfrak{p}_{i}}}}\left(\Lambda_{i}\right) \rightarrow G L_{\mathcal{O}_{F_{\mathfrak{p}_{i}}}}\left(\Lambda_{i} / \mathfrak{m}_{F_{\mathfrak{p}_{i}}}^{m_{i}} \Lambda_{i}\right)\right) .
$$

The corresponding moduli problem of sufficiently small level $U^{p}(\vec{m})$ over $\mathcal{O}_{K}$ is given by the functor

$$
\begin{gathered}
\left(\begin{array}{c}
\text { Connected, locally noetherian } \\
\mathcal{O}_{K} \text {-schemes with geometric point } \\
(S, s)
\end{array}\right) \rightarrow(\text { Sets }) \\
(S, s) \mapsto\left\{\left(A, \lambda, i, \bar{\eta}^{p},\left\{\alpha_{i}\right\}_{i=1}^{r}\right)\right\} / \sim
\end{gathered}
$$

where

- $A$ is an abelian scheme over $S$;

- $\lambda: A \rightarrow A^{\vee}$ is a prime-to- $p$ polarization;

- $i: \mathcal{O}_{F} \hookrightarrow \operatorname{End}(A) \otimes_{\mathbb{Z}} \mathbb{Z}_{(p)}$ such that $(A, i)$ is compatible and $\lambda \circ i(f)=$ $i\left(f^{*}\right)^{\vee} \circ \lambda, \forall f \in \mathcal{O}_{F}$

- $\bar{\eta}^{p}$ is a $\pi_{1}(S, s)$-invariant $U^{p}$-orbit of isomorphisms of Hermitian $F \otimes \mathbb{Q}$ $\mathbb{A}^{\infty, p}$-modules

$$
\eta: V \otimes_{\mathbb{Q}} \mathbb{A}^{\infty, p} \rightarrow V^{p} A_{s}
$$

which take the fixed pairing $\langle\cdot, \cdot\rangle$ on $V$ to an $\left(\mathbb{A}^{\infty, p}\right)^{\times}$-multiple of the $\lambda$ Weil pairing on $V A_{s}$. Here $V^{p} A_{s}$ is the adelic Tate module away from $p$

- for $i=1,2, \alpha_{i}: \mathfrak{p}_{i}^{-m_{i}} \Lambda_{i} / \Lambda_{i} \rightarrow A\left[\mathfrak{p}_{i}^{m_{i}}\right]$ is a Drinfeld $\mathfrak{p}_{i}^{m_{i}}$-structure, i.e. the 
set of $\alpha_{i}(x), x \in\left(\mathfrak{p}_{i}^{-m_{i}} \Lambda_{i} / \Lambda_{i}\right)$ forms a full set of sections of $A\left[\mathfrak{p}_{i}^{m_{i}}\right]$ in the sense of section 1.8 of $[\mathrm{KM}]$;

- for $i>2, \alpha_{i}:\left(\mathfrak{p}_{i}^{-m_{i}} \Lambda_{i} / \Lambda\right) \stackrel{\sim}{\rightarrow} A\left[\mathfrak{p}_{i}^{m_{i}}\right]$ is an isomorphism of $S$-schemes with $\mathcal{O}_{F_{\mathfrak{p}_{i}}}$-actions;

- Two tuples $\left(A, \lambda, i, \bar{\eta}^{p},\left\{\alpha_{i}\right\}_{i=1}^{r}\right)$ and $\left(A^{\prime}, \lambda^{\prime}, i^{\prime},\left(\bar{\eta}^{p}\right)^{\prime},\left\{\alpha_{i}^{\prime}\right\}_{i=1}^{r}\right.$ are equivalent if there is a prime-to-p isogeny $A \rightarrow A^{\prime}$ taking $\lambda, i, \bar{\eta}^{p}, \alpha_{i}$ to $\gamma \lambda^{\prime}, i^{\prime},\left(\bar{\eta}^{p}\right)^{\prime}, \alpha_{i}^{\prime}$ for some $\gamma \in \mathbb{Z}_{(p)}^{\times}$.

This moduli problem is representable by a projective scheme over $\mathcal{O}_{K}$, which will be denoted $X_{U^{p}, \vec{m}}$. The projectivity follows from Theorem 5.3.3.1 and Remark 5.3.3.2 of [Lan]. If $m_{1}=m_{2}=0$ this scheme is smooth as in Lemma III.4.1.2 of $[\mathrm{HT}]$, since we can check smoothness on the completed strict local rings at closed geometric points and these are isomorphic to deformation rings for $p$-divisible groups (with level structure only at $\mathfrak{p}_{i}$ for $i>2$, when the $p$-divisible group is etale). Moreover, if $m_{1}=m_{2}=0$ the dimension of $X_{U^{p}, \vec{m}}$ is $2 n-1$.

When $m_{1}=m_{2}=0$, we will denote $X_{U^{p}, \vec{m}}$ by $X_{U_{0}}$. If $\mathcal{A}_{U_{0}}$ is the universal abelian scheme over $X_{U_{0}}$ we write $\mathcal{G}_{i}=\mathcal{A}_{U_{0}}\left[\mathfrak{p}_{i}^{\infty}\right]$ for $i=1,2$ and $\mathcal{G}=\mathcal{G}_{1} \times \mathcal{G}_{2}$. Over a base where $p$ is nilpotent, each of the $\mathcal{G}_{i}$ is a one-dimensional compatible Barsotti-Tate $\mathcal{O}_{K}$-module.

Let $\mathbb{F}$ be the residue field of $\mathcal{O}_{K}$. Let $\bar{X}_{U_{0}}=X_{U_{0}} \times_{\text {Spec } \mathcal{O}_{K}}$ Spec $\mathbb{F}$ be the special fiber of $X_{U_{0}}$. We define a stratification on $\bar{X}_{U_{0}}$ in terms of $0 \leq h_{1}, h_{2}<$ $n-1$. The scheme $\bar{X}_{U_{0}}^{\left[h_{1}, h_{2}\right]}$ will be the reduced closed subscheme of $\bar{X}_{U_{0}}$ whose closed geometric points $s$ are those for which the maximal etale quotient of $\mathcal{G}_{i}$ has $\mathcal{O}_{K}$-height at most $h_{i}$. Let $\bar{X}_{U_{0}}^{\left(h_{1}, h_{2}\right)}=\bar{X}_{U_{0}}^{\left[h_{1}, h_{2}\right]}-\left(\bar{X}_{U_{0}}^{\left[h_{1}-1, h_{2}\right]} \cup \bar{X}_{U_{0}}^{\left[h_{1}, h_{2}-1\right]}\right)$.

Lemma 2.2.2. The scheme $\bar{X}_{U_{0}}^{\left(h_{1}, h_{2}\right)}$ is non-empty and smooth of pure dimension $h_{1}+h_{2}$.

Proof. In order to see that this is true, note that the formal completion of $\bar{X}_{U_{0}}$ 
at any closed point is isomorphic to $\overline{\mathbb{F}}\left[\left[T_{2}, \ldots, T_{n}, S_{2}, \ldots, S_{n}\right]\right]$ since it is the universal formal deformation ring of a product of two one-dimensional compatible Barsotti-Tate groups of height $n$ each. (In fact it is the product of the universal deformation rings for each of the two Barsotti-Tate groups.) Thus, $\bar{X}_{U_{0}}$ has dimension $2 n-2$ and as in Lemma II.1.1 of [HT] each closed stratum $\bar{X}_{U_{0}}^{\left[h_{1}, h_{2}\right]}$ has dimension at least $h_{1}+h_{2}$. The lower bound on dimension also holds for each open stratum $\bar{X}_{U_{0}}^{\left(h_{1}, h_{2}\right)}$. In order to get the upper bound on the dimension it suffices to show that the lowest stratum $\bar{X}_{U_{0}}^{(0,0)}$ is non-empty. Indeed, once we have a closed point $s$ in any stratum $\bar{X}_{U_{0}}^{\left(h_{1}, h_{2}\right)}$, we can compute the formal completion $\left(\bar{X}_{U_{0}}^{\left(h_{1}, h_{2}\right)}\right)_{s}^{\wedge}$ as in Lemma II.1.3 of [HT] and find that the dimension is exactly $h_{1}+h_{2}$. We start with a closed point of the lowest stratum $\bar{X}_{U_{0}}^{(0,0)}=\bar{X}_{U_{0}}^{[0,0]}$ and prove that this stratum has dimension 0 . The higher closed strata $\bar{X}_{U_{0}}^{\left[h_{1}, h_{2}\right]}=\cup_{j_{1} \leq h_{1}, j_{2} \leq h_{2}} \bar{X}_{U_{0}}^{\left(j_{1}, j_{2}\right)}$ are non-empty and it follows by induction on $\left(h_{1}, h_{2}\right)$ that the open strata $\bar{X}_{U_{0}}^{\left(h_{1}, h_{2}\right)}$ are also non-empty.

It remains to see that $\bar{X}_{U_{0}}^{(0,0)}$ is non-empty. This can be done using HondaTate theory as in the proof of Corollary V.4.5. of [HT], whose ingredients for Shimura varieties associated to more general unitary groups are supplied in sections 8 through 12 of [Sh1]. In our case, Honda-Tate theory exhibits a bijection between $p$-adic types over $F$ (see section 8 of [Sh1] for the general definition) and pairs $(A, i)$ where $A / \overline{\mathbb{F}}$ is an abelian variety of dimension $d n$ and $i: F \hookrightarrow \operatorname{End}(A) \otimes_{\mathbb{Z}} \mathbb{Q}$. The abelian variety $A$ must also satisfy the following: $A\left[\mathfrak{p}_{i}^{\infty}\right]$ is ind-etale for $i>2$ and $A\left[\mathfrak{p}_{i}^{\infty}\right]$ is one-dimensional of etale height $h_{i}$ for $i=1,2$. Note that the slopes of the p-divisible groups $A\left[\mathfrak{p}_{i}^{\infty}\right]$ are fixed for all $i$. All our $p$-adic types will be simple and given by pairs $(M, \eta)$ where $M$ is a $\mathrm{CM}$ field extension of $F$ and $\eta \in \mathbb{Q}[\mathfrak{P}]$ where $\mathfrak{P}$ is the set of places of $M$ above $p$. The coefficients in $\eta$ of places $x$ of $M$ above $\mathfrak{p}_{i}$ are related to the slope of the corresponding $p$-divisible group at $\mathfrak{p}_{i}$ as in Corollary 8.5 of [Sh3]. More 
precisely, $A\left[x^{\infty}\right]$ has pure slope $\eta_{x} / e_{x / p}$. It follows that the coefficients of $\eta$ at places $x$ and $x^{c}$ above $p$ satisfy the compatibility

$$
\eta_{x}+\eta_{x^{c}}=e_{x / p}
$$

so to know $\eta$ it is enough to specify $\eta_{x} \cdot x$ as $x$ runs through places of $M$ above $u$.

In order to exhibit a pair $(A, i)$ with the right slope of $A\left[\mathfrak{p}_{i}^{\infty}\right]$ it suffices to exhibit its corresponding $p$-adic type. For this, we can simply take $M=F$ and $\eta_{\mathfrak{p}_{i}}=\frac{e_{\mathfrak{p}_{i} / p}}{n\left[F_{\mathfrak{p}_{i}}: \mathbb{Q}_{p}\right]} \cdot \mathfrak{p}_{i}$ for $i=1,2$ and $\eta_{\mathfrak{p}_{i}}=0$ otherwise. The only facts remaining to be checked are that the associated pair $(A, i)$ has a polarization $\lambda$ which induces $c$ on $F$ and that the triple $(A, i, \lambda)$ can be given additional structure to make it into a point on $\bar{X}_{U_{0}}^{(0,0)}$. First we endow $(A, i)$ with a polarization $\lambda_{0}$ for which the Rosati involution induces $c$ on $F$ using Lemma 9.2 of [Ko1] and we use Lemma 5.2.1, an analogue of Lemma V.4.1 of [HT], to construct an $F$-module $W_{0}$ together with a non-degenerate Hermitian pairing such that

$$
W_{0} \otimes \mathbb{A}^{\infty, p} \simeq V^{p} A \text { and } W_{0} \otimes_{\mathbb{Q}} \mathbb{R} \simeq V \otimes_{\mathbb{Q}} \mathbb{R}
$$

as Hermitian $F \otimes_{\mathbb{Q}} \mathbb{A}^{\infty, p}$-modules $\left(F \otimes_{\mathbb{Q}} \mathbb{R}\right.$-modules respectively). Then we use the difference (in the Galois cohomology sense) between $W_{0}$ and $V$ as Hermitian $F$-modules over $\mathbb{Q}$ to find a polarization $\lambda$ such that $V^{p} A$ with its $\lambda$-Weil pairing is equivalent to $V \otimes \mathbb{A}^{\infty, p}$ with its standard pairing, as in Lemma V.4.3 of $[\mathrm{HT}]$. Note that the argument is not circular, since the proof of Lemma 5.2.1 is independent of this section.

The next Lemma is an analogue of Lemma 3.1 of [TY].

Lemma 2.2.3. If $0 \leq h_{1}, h_{2} \leq n-1$ then the Zariski closure of $\bar{X}_{U_{0}}^{\left(h_{1}, h_{2}\right)}$ contains $\bar{X}_{U_{0}}^{(0,0)}$. 
Proof. The proof follows exactly like the proof of Lemma 3.1 of [TY]. Let $x$ be a closed geometric point of $\bar{X}_{U_{0}}^{(0,0)}$. The main point is to note that the formal completion of $\bar{X}_{U_{0}} \times$ Spec $\overline{\mathbb{F}}$ at $x$ is isomorphic to the equicharacteristic universal deformation ring of $\mathcal{G}_{1, x} \times \mathcal{G}_{2, x}$, so it is isomorphic to

$$
\text { Spf } \overline{\mathbb{F}}\left[\left[T_{2}, \ldots, T_{n}, S_{2}, \ldots, S_{n}\right]\right] \text {. }
$$

We can choose the $T_{i}$, the $S_{i}$ and formal parameters $X$ on the universal deformation of $\mathcal{G}_{1, x}$ and $Y$ on the universal deformation of $\mathcal{G}_{2, x}$ such that

$$
\begin{gathered}
{[\pi](X) \equiv \pi X+\sum_{i=2}^{n} T_{i} X^{\# \mathbb{F}^{i-1}}+X^{\# \mathbb{F}^{n}} \quad\left(\bmod X^{\# \mathbb{F}^{n}+1}\right) \text { and }} \\
{[\pi](Y) \equiv \pi Y+\sum_{i=2}^{n} S_{i} X^{\# \mathbb{F}^{i-1}}+S^{\# \mathbb{F}^{n}} \quad\left(\bmod S^{\# \mathbb{F}^{n}+1}\right) .}
\end{gathered}
$$

We get a morphism

$$
\text { Spec } \overline{\mathbb{F}}\left[\left[T_{2}, \ldots, T_{n}, S_{2}, \ldots, S_{n}\right]\right] \rightarrow \bar{X}_{U_{0}}
$$

lying over $x:$ Spec $\overline{\mathbb{F}} \rightarrow \bar{X}_{U_{0}}$ such that if $k$ denotes the algebraic closure of the field of fractions of

$$
\text { Spec } \overline{\mathbb{F}}\left[\left[T_{2}, \ldots, T_{n}, S_{2}, \ldots S_{n}\right]\right] /\left(T_{2}, \ldots, T_{n-h_{1}}, S_{2}, \ldots, S_{n-h_{2}}\right)
$$

then the induced map Spec $k \rightarrow \bar{X}_{U_{0}}$ factors through $\bar{X}_{U_{0}}^{\left(h_{1}, h_{2}\right)}$.

For $i=1,2$, let $\operatorname{Iw}_{n, \mathfrak{p}_{i}}$ be the subgroup of matrices in $G L_{n}\left(\mathcal{O}_{K}\right)$ which reduce modulo $\mathfrak{p}_{i}$ to $B_{n}(\mathbb{F})$ (here $B_{n}(\mathbb{F}) \subset G L_{n}(\mathbb{F})$ is the Borel subgroup). We will define an integral model for $X_{U}$, where $U \subseteq G\left(\mathbb{A}^{\infty}\right)$ is equal to

$$
U^{p} \times U_{p}^{\mathfrak{p}_{1}, \mathfrak{p}_{2}}(\vec{m}) \times \operatorname{Iw}_{n, \mathfrak{p}_{1}} \times \operatorname{Iw}_{n, \mathfrak{p}_{2}} \times \mathbb{Z}_{p}^{\times}
$$


We define the following functor $\mathfrak{X}_{U}$ from connected locally noetherian $\mathcal{O}_{K^{-}}$ schemes with a geometric point to sets sending

$$
(S, s) \mapsto\left(A, \lambda, i, \bar{\eta}^{p}, \mathcal{C}_{1}, \mathcal{C}_{2}, \alpha_{i}\right)
$$

where $\left(A, \lambda, i, \bar{\eta}^{p}, \alpha_{i}\right)$ is as in the definition of $X_{U_{0}}$ and for $i=1,2, \mathcal{C}_{i}$ is a chain of isogenies

$$
\mathcal{C}_{i}: \mathcal{G}_{i, A}=\mathcal{G}_{i, 0} \rightarrow \mathcal{G}_{i, 1} \rightarrow \cdots \rightarrow \mathcal{G}_{i, n}=\mathcal{G}_{i, A} / \mathcal{G}_{i, A}\left[\mathfrak{p}_{i}\right]
$$

of compatible Barsotti-Tate $\mathcal{O}_{K}$-modules each of degree $\# \mathbb{F}$ and with composite the canonical map $\mathcal{G}_{i, A} \rightarrow \mathcal{G}_{i, A} / \mathcal{G}_{i . A}\left[\mathfrak{p}_{i}\right]$.

Lemma 2.2.4. If $U^{p}$ is sufficiently small, the functor $\mathfrak{X}_{U}$ is represented by a scheme $X_{U}$ which is finite over $X_{U_{0}}$. The scheme $X_{U}$ has some irreducible components of dimension $2 n-1$.

Proof. The chains of isogenies $\mathcal{C}_{i}$ can be viewed as flags

$$
0=\mathcal{K}_{i, 0} \subset \mathcal{K}_{i, 1} \cdots \subset \mathcal{K}_{i, n}=\mathcal{G}_{i}\left[\mathfrak{p}_{i}\right]
$$

where $\mathcal{K}_{i, j}=\operatorname{ker}\left(\mathcal{G}_{i, 0} \rightarrow \mathcal{G}_{i, j}\right)$. All the $\mathcal{K}_{i, j}$ are closed finite flat subgroup schemes with $\mathcal{O}_{K^{-}}$action and $\mathcal{K}_{i, j} / \mathcal{K}_{i, j-1}$ of order $\# \mathbb{F}$. The representability can be proved in the same way as in Lemma 3.2 of [TY] except in two steps: first we note that the functor sending $S$ to points of $X_{U_{0}}(S)$ together with flags $\mathcal{C}_{1}$ of $\mathcal{G}_{1}\left[\mathfrak{p}_{1}\right]$ is representable by a scheme $X_{U}^{\prime}$ over $X_{U_{0}}$. (If we let $\mathcal{H}_{1}$ denote the sheaf of Hopf algebras over $X_{U_{0}}$ defining $\mathcal{G}_{1}\left[\mathfrak{p}_{1}\right]$, then $X_{U}^{\prime}$ will be a closed subscheme of the Grassmanian of chains of locally free direct summands of $\mathcal{H}_{1}$.) Then we see in the same way that the functor sending $S$ to points of $X_{U}^{\prime}(S)$ together with flags $\mathcal{C}_{2}$ of $\mathcal{G}_{2}\left[\mathfrak{p}_{2}\right]$ is representable by a scheme $X_{U}$ over $X_{U}^{\prime}$. We also have 
that $X_{U}$ is projective and finite over $X_{U_{0}}$. (Indeed, for each closed geometric point $x$ of $X_{U_{0}}$ there are finitely many choices of flags of $\mathcal{O}_{K}$-submodules of each $\left.\mathcal{G}_{i, x}.\right)$ On the generic fiber, the morphism $X_{U} \rightarrow X_{U_{0}}$ is finite etale and $X_{U_{0}}$ has dimension $2 n-1$, so $X_{U}$ has some components of dimension $2 n-1$.

We say that an isogeny $\mathcal{G} \rightarrow \mathcal{G}^{\prime}$ of one-dimensional compatible Barsotti-Tate $\mathcal{O}_{K}$-modules of degree $\# \mathbb{F}$ has connected kernel if it induces the zero map on Lie $\mathcal{G}$. If we let $f=\left[\mathbb{F}: \mathbb{F}_{p}\right]$ and $F: \mathcal{G} \rightarrow \mathcal{G}^{(p)}$ be the Frobenius map, then $F^{f}: \mathcal{G} \rightarrow \mathcal{G}^{(\# \mathbb{F})}$ is an isogeny of one-dimensional compatible Barsotti-Tate $\mathcal{O}_{K^{-}}$ modules and has connected kernel. The following lemma appears as Lemma 3.3 in $[\mathrm{TY}]$.

Lemma 2.2.5. Let $W$ denote the ring of integers of the completion of the maximal unramified extension of $K$. Suppose that $R$ is an Artinian local $W$ algebra with residue field $\overline{\mathbb{F}}$. Suppose that

$$
\mathcal{C}: \mathcal{G}_{0} \rightarrow \mathcal{G}_{1} \rightarrow \cdots \rightarrow \mathcal{G}_{g}=\mathcal{G}_{0} / \mathcal{G}_{0}\left[\mathfrak{p}_{i}\right]
$$

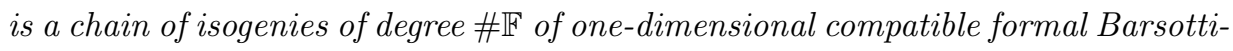
Tate $\mathcal{O}_{K}$-modules over $R$ of $\mathcal{O}_{K}$-height $g$ with composite equal to multiplication by $\pi$. If every isogeny has connected kernel then $R$ is a $\overline{\mathbb{F}}$-algebra and $\mathcal{C}$ is the pullback of a chain of isogenies of Barsotti-Tate $\mathcal{O}_{K}$-modules over $\overline{\mathbb{F}}$, with all isogenies isomorphic to $F^{f}$.

Now let $\bar{X}_{U}=X_{U} \times_{\text {Spec } K}$ Spec $\mathbb{F}$ denote the special fiber of $X_{U}$. For $i=1,2$ and $1 \leq j \leq n$, let $Y_{i, j}$ denote the closed subscheme of $\bar{X}_{U}$ over which $\mathcal{G}_{i, j-1} \rightarrow \mathcal{G}_{i, j}$ has connected kernel. Note that, since each Lie $\mathcal{G}_{i, j}$ is locally free of rank 1 over $\mathcal{O}_{X_{U}}$, we can pick a local basis for all of them. Then we can find locally $X_{i, j} \in \Gamma\left(X_{U}, O_{X_{U}}\right)$ to represent the linear maps $\mathrm{Lie}_{i, j-1} \rightarrow \mathrm{Lie} \mathcal{G}_{i, j}$. Thus, each $Y_{i, j}$ is cut out locally in $X_{U}$ by the equation $X_{i, j}=0$. 
Proposition 2.2.6. Let $s$ be a closed geometric point of $X_{U}$ such that $\mathcal{G}_{i, s}$ has etale height $h_{i}$ for $i=1,2$. Let $W$ be the ring of integers of the completion of the maximal unramified extension of $K$. Let $\mathcal{O}_{X_{U}, s}^{\wedge}$ be the completion of the strict henselization of $X_{U}$ at s, i.e. the completed local ring of $X \times{ }_{\text {Spec }} \mathcal{O}_{K}$ Spec $W$ at $s$. Then

$$
\mathcal{O}_{X_{U}, s}^{\wedge} \simeq W\left[\left[T_{1}, \ldots, T_{n}, S_{1}, \ldots, S_{n}\right]\right] /\left(\prod_{i=h_{1}+1}^{n} T_{i}-\pi, \prod_{i=h_{2}+1}^{n} S_{i}-\pi\right) .
$$

Assume that $Y_{1, j_{k}}$ for $k=1, \ldots, n-h_{1}$ and $j_{k} \in\{1, \ldots, n\}$ distinct are subschemes of $X_{U}$ which contain s as a geometric point. We can choose the generators $T_{i}$ such that the completed local ring $\mathcal{O}_{Y_{1, j_{k}, s}}^{\wedge}$ is cut out in $\mathcal{O}_{X_{U}, s}^{\wedge}$ by the equation $T_{k+h_{1}}=0$. The analogous statement is true for $Y_{2, j_{k}}$ with $k=1, \ldots, n-h_{2}$ and $S_{k+h_{2}}=0$.

Proof. First we prove that $X_{U}$ has pure dimension $2 n-1$ by using Deligne's homogeneity principle. We will follow closely the proof of Proposition 3.4.1 of [TY]. The dimension of $\mathcal{O}_{X_{U}, s}^{\wedge}$ as $s$ runs over geometric points of $X_{U}$ above $\bar{X}_{U_{0}}^{(0,0)}$ is constant, say it is equal to $m$. Then we claim that $\mathcal{O}_{X_{U}, s}^{\wedge}$ has dimension $m$ for every closed geometric point of $X_{U}$. Indeed, assume the subset of $X_{U}$ where $\mathcal{O}_{X_{U}, s}^{\wedge}$ has dimension different from $m$ is non-empty. Then this subset is closed, so its projection to $X_{U_{0}}$ is also closed and so it must contain some $\bar{X}_{U_{0}}^{\left(h_{1}, h_{2}\right)}$ (since the dimension of $\mathcal{O}_{X_{U}, s}^{\wedge}$ only depends on the stratum of $X_{U_{0}}$ that $s$ is above). By Lemma 2.2.3, the closure of $\bar{X}_{U_{0}}^{\left(h_{1}, h_{2}\right)}$ contains $\bar{X}_{U_{0}}^{(0,0)}$, which is a contradiction. Thus, $X_{U}$ has pure dimension $m$ and by Lemma 2.2.4, $m=2 n-1$.

The completed local ring $\mathcal{O}_{X_{U}, s}^{\wedge}$ is the universal deformation ring for tuples $\left(A, \lambda, i, \bar{\eta}^{p}, \mathcal{C}_{1}, \mathcal{C}_{2}, \alpha_{i}\right)$ deforming $\left(A_{s}, \lambda_{s}, i_{s}, \bar{\eta}_{s}^{p}, \mathcal{C}_{1, s}, \mathcal{C}_{2, s}, \alpha_{i, s}\right)$. Deforming the abelian variety $A_{s}$ is the same as deforming its $p$-divisible group $A_{s}\left[p^{\infty}\right]$ by Serre-Tate and $A_{s}\left[p^{\infty}\right]=A_{s}\left[u^{\infty}\right] \times A_{s}\left[\left(u^{c}\right)^{\infty}\right]$. The polarization $\lambda$ together with 
$A\left[u^{\infty}\right]$ determine $A\left[\left(u^{c}\right)^{\infty}\right]$, so it suffices to deform $A_{s}\left[u^{\infty}\right]$ as an $\mathcal{O}_{F}$-module together with the level structure. At primes other than $\mathfrak{p}_{1}$ and $\mathfrak{p}_{2}$, the $p$-divisible group is etale, so the deformation is uniquely determined. Moreover, $A\left[\left(\mathfrak{p}_{1} \mathfrak{p}_{2}\right)^{\infty}\right]$ decomposes as $A\left[\mathfrak{p}_{1}^{\infty}\right] \times A\left[\mathfrak{p}_{2}^{\infty}\right]$ (because $\mathcal{O}_{F} \otimes_{\mathcal{O}_{F^{\prime}}} \mathcal{O}_{F_{\mathfrak{p}_{1} \mathfrak{p}_{2}}^{\prime}} \simeq \mathcal{O}_{F, \mathfrak{p}_{1}} \times \mathcal{O}_{F, \mathfrak{p}_{2}}$ ), so it suffices to consider deformations of the chains

$$
\mathcal{C}_{i, s}: \mathcal{G}_{i, s}=\mathcal{G}_{i, 0} \rightarrow \mathcal{G}_{i, 1} \rightarrow \cdots \rightarrow \mathcal{G}_{i, n}=\mathcal{G}_{i, s} / \mathcal{G}_{i, s}\left[\mathfrak{p}_{i}\right]
$$

for $i=1,2$ separately.

Let $\mathcal{G} \simeq \Sigma \times\left(K / \mathcal{O}_{K}\right)^{h}$ be a $p$-divisible $\mathcal{O}_{K}$-module over $\overline{\mathbb{F}}$ of dimension one and total height $n$. Let

$$
\mathcal{C}: \mathcal{G}=\mathcal{G}_{0} \rightarrow \mathcal{G}_{1} \rightarrow \cdots \rightarrow \mathcal{G}_{n}=\mathcal{G} / \mathcal{G}[\pi]
$$

be a chain of isogenies of degree $\# \mathbb{F}$. Since we are working over $\overline{\mathbb{F}}$, the chain $\mathcal{C}$ splits into a formal part and an etale part. Let $\mathcal{C}^{0}$ be the chain obtained from $\mathcal{C}$ by restricting it to the formal part:

$$
\tilde{\Sigma} \rightarrow \tilde{\Sigma}_{1} \rightarrow \cdots \rightarrow \tilde{\Sigma}_{n}=\tilde{\Sigma} / \tilde{\Sigma}[\pi]
$$

Let $J \subseteq\{1, \ldots, n\}$ be the subset of indices $j$ for which $\mathcal{G}_{j-1} \rightarrow \mathcal{G}_{j}$ has connected kernel. (The cardinality of $J$ is $n-h$.) Also assume that the chain $\mathcal{C}^{\text {et }}$ consists of

$$
\mathcal{G}_{j}^{\text {et }}=\left(K / \pi^{-1} \mathcal{O}_{K}\right)^{j} \oplus\left(K / \mathcal{O}_{K}\right)^{h-j}
$$

for all $j \in J$ with the obvious isogenies between them.

We claim that the universal deformation $\operatorname{ring}$ of $\mathcal{C}$ is isomorphic to

$$
W\left[\left[T_{1}, \ldots, T_{n}\right]\right] /\left(\prod_{j \in J} T_{j}-\pi\right) .
$$


We will follow the proof of Proposition 4.5 of [D]. To see the claim, we first consider deformations of $\mathcal{G}$ without level structure. By proposition 4.5 of [D], the universal deformation ring of $\Sigma$ is

$$
R^{0} \simeq W\left[\left[X_{h+1}, \ldots, X_{h}\right]\right] /\left(X_{h+1} \cdots X_{n}-\pi\right)
$$

Let $\tilde{\Sigma}$ be the universal deformation of $\Sigma$. By considering the connected-etale exact sequence, we see that the deformations of $\mathcal{G}$ are classified by extensions of the form

$$
0 \rightarrow \tilde{\Sigma} \rightarrow \tilde{\mathcal{G}} \rightarrow\left(K / \mathcal{O}_{K}\right)^{h} \rightarrow 0
$$

Thus, the universal deformations of $\mathcal{G}$ are classified by elements of $\operatorname{Hom}(T \mathcal{G}, \tilde{\Sigma})$, where $T \mathcal{G}$ is the Tate module of $\mathcal{G}$. The latter ring is non-canonically isomorphic to

$$
R \simeq W\left[\left[X_{1}, \ldots, X_{n}\right]\right] /\left(\prod_{j \in J} X_{j}-\pi\right) .
$$

Let $S$ be the universal deformation ring for deformations of the chain $\mathcal{C}$ and $S^{0}$ be the universal deformation ring for the chain $\mathcal{C}^{0}$. Let

$$
\tilde{\mathcal{C}}: \tilde{\mathcal{G}}=\tilde{\mathcal{G}}_{0} \rightarrow \tilde{\mathcal{G}}_{1} \rightarrow \cdots \rightarrow \tilde{\mathcal{G}}_{n}=\tilde{\mathcal{G}} / \tilde{\mathcal{G}}[\pi]
$$

be the universal deformation of $\mathcal{C}$ which corresponds when restricted to the formal part to the universal chain

$$
\tilde{\Sigma} \rightarrow \tilde{\Sigma}_{1} \rightarrow \cdots \rightarrow \tilde{\Sigma}_{n}=\tilde{\Sigma} / \tilde{\Sigma}[\pi]
$$

Each deformation $\tilde{\mathcal{G}}_{j}$ of $\mathcal{G}_{j}$ is defined by a connected-etale exact sequence

$$
0 \rightarrow \tilde{\Sigma}_{j} \rightarrow \tilde{\mathcal{G}}_{j} \rightarrow\left(K / \mathcal{O}_{K}\right)^{h} \rightarrow 0
$$


so by an element $f_{j} \in \operatorname{Hom}\left(T \mathcal{G}_{j}, \tilde{\Sigma}_{j}\right)$. We will explore the compatibilities between the $\operatorname{Hom}\left(T \mathcal{G}_{j}, \tilde{\Sigma}_{j}\right)$ as $j$ ranges from 0 to $n$. If $j \in J$ then $\tilde{\mathcal{G}}_{j-1} \rightarrow \tilde{\mathcal{G}_{j}}$ has connected kernel, so $T \mathcal{G}_{j-1} \simeq T \mathcal{G}_{j}$. The isogeny $\tilde{\Sigma}_{j-1} \rightarrow \tilde{\Sigma}_{j}$ determines a map $\operatorname{Hom}\left(T \mathcal{G}_{j-1}, \tilde{\Sigma}_{j-1}\right) \rightarrow \operatorname{Hom}\left(T \mathcal{G}_{j}, \tilde{\Sigma}_{j}\right)$, which determines the extension $\tilde{\mathcal{G}}_{j}$. Thus, in order to know the extension classes of $\tilde{\mathcal{G}}_{j}$ it suffices to focus on the case $j \notin J$.

Let $\left(e_{j}\right)_{j \in J}$ be a basis of $\mathcal{O}_{K}^{h}$, which we identify with $T \mathcal{G}_{j}$ for each $j$. We claim that it suffices to know $f_{j}\left(e_{j}\right) \in \tilde{\Sigma}_{j}$ for each $j \notin J$. Indeed, if $j \notin J$ then we know that $\tilde{\Sigma}_{j-1} \simeq \tilde{\Sigma}_{j}$ and we also have a map $T \mathcal{G}_{j-1} \rightarrow T \mathcal{G}_{j}$ sending

$$
e_{j^{\prime}} \mapsto e_{j^{\prime}} \text { for } j^{\prime} \neq j \text { and } e_{j} \mapsto \pi e_{j}
$$

Thus, for $i \neq j$ we can identify $f_{j-1}\left(e_{i}\right) \in \tilde{\Sigma}_{j-1}$ with $f_{j}\left(e_{i}\right) \in \tilde{\Sigma}_{j}$. Hence if we know $f_{j}\left(e_{j}\right)$ then we also know $f_{j^{\prime}}\left(e_{j}\right)$ for all $j^{\prime}>j$. Thus we know $f_{n}\left(e_{j}\right)$, but recall that $f_{n}$ corresponds to the extension

$$
0 \rightarrow \tilde{\Sigma} / \tilde{\Sigma}[\pi] \rightarrow \tilde{\mathcal{G}} / \tilde{\mathcal{G}}[\pi] \rightarrow\left(K / \pi^{-1} \mathcal{O}_{K}\right)^{h} \rightarrow 0
$$

which is isomorphic to the extension

$$
0 \rightarrow \tilde{\Sigma} \rightarrow \tilde{\mathcal{G}} \rightarrow\left(K / \mathcal{O}_{K}\right)^{h} \rightarrow 0
$$

Therefore we also know $f_{0}\left(e_{j}\right)$ and by extension all $f_{j^{\prime}}\left(e_{j}\right)$ for $j^{\prime}<j$. This proves the claim that the only parameters needed to construct all the extensions $\tilde{\mathcal{G}}_{j}$ are the elements $f_{j}\left(e_{j}\right) \in \tilde{\Sigma}_{j}$ for all $j \notin J$.

We have a map $S^{0} \otimes_{R^{0}} R \rightarrow S$ induced by restricting the Iwahori level structure to the formal part. From the discussion above, we see that this map is finite and that $S$ is obtained from $S^{0} \otimes_{R^{0}} R$ by adjoining for each $j \in J$ a 
root $T_{j}$ of

$$
f\left(T_{j}\right)=X_{j}
$$

in $\tilde{\Sigma}$, where $f: \tilde{\Sigma} \rightarrow \tilde{\Sigma}$ is the composite of the isogenies $\tilde{\Sigma}_{j} \rightarrow \tilde{\Sigma}_{j+1} \rightarrow \cdots \rightarrow \tilde{\Sigma}_{n}$. If we quotient $S$ by all the $T_{j}$ for $j \notin J$, we are left only with deformations of the chain $\mathcal{C}^{0}$, since all of the connected-etale exact sequences will split. Thus $S /\left(T_{j}\right)_{j \notin J} \simeq S^{0}$.

Now, the formal part $\tilde{\mathcal{C}}^{0}$ can be written as a chain

$$
\tilde{\Sigma}=\tilde{\Sigma}_{0} \rightarrow \cdots \rightarrow \tilde{\Sigma}_{j} \rightarrow \cdots \rightarrow \tilde{\Sigma} / \tilde{\Sigma}[\pi]
$$

of length $n-h$. Choose bases $e_{j}$ for Lie $\mathcal{G}_{j}$ over $S^{0}$ as $j$ runs over $J$, such that

$$
e_{n}=e_{j} \text { for the largest } j \in J
$$

maps to

$$
e_{0}=e_{j} \text { for the smallest } j \in J
$$

under the isomorphism $\mathcal{G}_{n}=\mathcal{G}_{0} / \mathcal{G}_{0}[\pi] \stackrel{\sim}{\rightarrow} \mathcal{G}_{0}$ induced by $\pi$. Let $T_{j} \in S^{0}$ represent the linear map Lie $\tilde{\Sigma}_{j^{\prime}} \rightarrow$ Lie $\tilde{\Sigma}_{j}$, where $j^{\prime}$ is the largest element of $J$ for which $j^{\prime}<j$. Then

$$
\prod_{j \in J} T_{j}=\pi .
$$

Moreover, $S^{0} /\left(T_{j}\right)_{j \in J}=\overline{\mathbb{F}}$ by Lemma 2.2.5. (See also the proof of Proposition 3.4 of $[\mathrm{TY}]$.$) Hence we have a surjection$

$$
W\left[\left[T_{1}, \ldots, T_{n}\right]\right] /\left(\prod_{j=h_{1}+1}^{n} T_{j}-\pi\right) \rightarrow S,
$$

which by dimension reasons must be an isomorphism.

Applying the preceding argument to the chains $\mathcal{C}_{1, s}$ and $\mathcal{C}_{2, s}$, we conclude 
that

$$
\mathcal{O}_{X_{U}, s}^{\wedge} \simeq W\left[\left[T_{1}, \ldots, T_{n}, S_{1}, \ldots, S_{n}\right]\right] /\left(\prod_{i=h_{1}+1}^{n} T_{i}-\pi, \prod_{i=h_{2}+1}^{n} S_{i}-\pi\right)
$$

Moreover, the closed subvariety $Y_{1, j_{k}}$ of $X_{U}$ is exactly the locus where $\mathcal{G}_{j_{k}-1} \rightarrow$ $\mathcal{G}_{j_{k}}$ has connected kernel, so, if $s$ is a geometric point of $Y_{1, j_{k}}$, then $\mathcal{O}_{Y_{1, j_{k}}, s}^{\wedge}$ is cut out in $\mathcal{O}_{X_{U}, s}^{\wedge}$ by the equation $T_{k+h_{1}}=0$. (Indeed, by our choice of the parameters $T_{k+h_{1}}$ with $1 \leq k \leq n-h_{1}$, the condition that $\mathcal{G}_{1, j_{k}-1} \rightarrow \mathcal{G}_{1, j_{k}}$ has connected kernel is equivalent to $T_{k+h_{1}}=0$.)

For $S, T \subseteq\{1, \ldots, n\}$ non-empty let

$$
Y_{U, S, T}=\left(\bigcap_{i \in S} Y_{1, i}\right) \cap\left(\bigcap_{j \in T} Y_{2, j}\right)
$$

Then $Y_{U, S, T}$ is smooth over Spec $\mathbb{F}$ of pure dimension $2 n-\# S-\# T$ (we can check smoothness on completed local rings) and it is also proper over Spec $\mathbb{F}$, since $Y_{U, S, T} \hookrightarrow \bar{X}_{U}$ is a closed immersion and $\bar{X}_{U}$ is proper over Spec $\mathbb{F}$. We also define

$$
Y_{U, S, T}^{0}=Y_{U, S, T} \backslash\left(\left(\bigcup_{S^{\prime} \supsetneq S} Y_{U, S^{\prime}, T}\right) \cup\left(\bigcup_{T^{\prime} \supsetneq T} Y_{U, S, T^{\prime}}\right)\right) .
$$

Note that the inverse image of $\bar{X}_{U}^{\left(h_{1}, h_{2}\right)}$ with respect to the finite flat map $\bar{X}_{U} \rightarrow \bar{X}_{U_{0}}$ is

$$
\bigcup_{\substack{\# S=n-h_{1} \\ \# T=n-h_{2}}} Y_{U, S, T}^{0}
$$

Note that, when we consider the Shimura variety $X_{U_{i}}$, with $U_{i}$ having Iwahori level structure at only one of the primes $\mathfrak{p}_{i}$ for $i=1,2$, this will be flat over $X_{U_{0}}$, since it can be checked that it is a finite map between regular schemes 
of the same dimension (the same reason as in setting of [TY]). The morphism $X_{U} \rightarrow X_{U_{0}}$ is the fiber product of the morphisms $X_{U_{i}} \rightarrow X_{U_{0}}$ for $i=1,2$, so it is flat as well.

Lemma 2.2.7. The Shimura variety $X_{U}$ is locally etale over

$$
X_{r, s}=\operatorname{Spec} \mathcal{O}_{K}\left[X_{1}, \ldots, X_{n}, Y_{1}, \ldots Y_{n}\right] /\left(\prod_{i=1}^{r} X_{i}-\pi, \prod_{j-1}^{s} Y_{j}-\pi\right)
$$

with $1 \leq r, s \leq n$.

Proof. Let $x$ be a closed point of $X_{U}$. The completion of the strict henselization of $X_{U}$ at $x \mathcal{O}_{X_{U}, x}^{\wedge}$ is isomorphic to

$$
\mathcal{O}_{r, s}=W\left[\left[X_{1}, \ldots, X_{n}, Y_{1} \ldots, Y_{n}\right]\right] /\left(\prod_{i=1}^{r} X_{i}-\pi, \prod_{j=1}^{s} Y_{j}-\pi\right)
$$

for certain $1 \leq r, s \leq n$. We will show that there is an open affine neighbourhood $U$ of $x$ in $X$ such that $U$ is etale over $X_{r, s}$. Note that there are local equations $T_{i}=0$ with $1 \leq i \leq r$ and $S_{j}=0$ with $1 \leq j \leq s$ which define the closed subschemes $Y_{1, i}$ with $1 \leq i \leq r$ and $Y_{2, j}$ with $1 \leq j \leq s$ passing through $x$. Moreover, the parameters $T_{i}$ and $S_{j}$ satisfy

$$
\prod_{i=1}^{r} T_{i}=u \pi \text { and } \prod_{j=1}^{s} S_{i}=u^{\prime} \pi
$$

with $u$ and $u^{\prime}$ units in the local ring $\mathcal{O}_{X_{U}, x}$. We will explain why this is the case for the $T_{i}$. In the completion of the strict henselization $\mathcal{O}_{X_{U}, x}^{\wedge}$ both $T_{i}$ and $X_{i}$ cut out the completion of the strict henselization $\mathcal{O}_{Y_{1, i}, x}^{\wedge}$, which means that $T_{i}$ and $X_{i}$ differ by a unit. Taking the product of the $T_{i}$ we find that $\prod_{i=1}^{r} T_{i}=u \pi$ for $u \in \mathcal{O}_{X_{U}, x}^{\wedge}$ a unit in the completion of the strict henselization of the local ring. At the same time, in an open neighborhood of $x$, the special fiber of $X$ is a union of the divisors corresponding to $T_{i}=0$ for $1 \leq i \leq r$, so that $\prod_{i=1}^{r} T_{i}$ 
belongs to the ideal of $\mathcal{O}_{X_{U}, x}$ generated by $\pi$. We conclude that $u$ is actually a unit in the local ring $\mathcal{O}_{X_{U}, x}$, not only in $\mathcal{O}_{X_{U}, x}^{\wedge}$. In a neighborhood of $x$, we can change one of the $T_{i}$ by $u^{-1}$ and one of the $S_{i}$ by $\left(u^{\prime}\right)^{-1}$ to ensure that

$$
\prod_{i=1}^{r} T_{i}=\pi \text { and } \prod_{j=1}^{s} S_{i}=\pi .
$$

We will now adapt the argument used in the proof of Proposition 4.8 of [Y] to our situation. We first construct an unramified morphism $f$ from a neighborhood of $x$ in $X_{U}$ to Spec $\mathcal{O}_{K}\left[X_{1}, \ldots, X_{n}, Y_{1}, \ldots Y_{n}\right]$. We can do this simply by sending the $X_{i}$ to the $T_{i}$ for $i=1, \ldots r$ and the $Y_{j}$ to the $S_{j}$ for $j=1, \ldots s$. The rest of the $X_{i}$ and $Y_{j}$ can be sent to parameters in a neighborhood of $x$ which approximate the remaining parameters in $\mathcal{O}_{X_{U}, x}^{\wedge}$ modulo the square of the maximal ideal. Then $f$ will be formally unramified at the point $x$. By [EGA4] 18.4.7 we see that when restricted to an open affine neighbourhood Spec $A$ of $x$ in $X,\left.f\right|_{\operatorname{Spec} A}$ can be decomposed as a closed immersion Spec $A \rightarrow \operatorname{Spec} B$ followed by an etale morphism $\operatorname{Spec} B \rightarrow \operatorname{Spec} \mathcal{O}_{K}\left[X_{1}, \ldots X_{n}, Y_{1}, \ldots, Y_{n}\right]$. The closed immersion translates into the fact that $A \simeq B / I$ for some ideal $I$ of $B$. The inverse image of $I$ in $W\left[X_{1}, \ldots, X_{n}, Y_{1}, \ldots Y_{n}\right]$ is an ideal $J$ which contains $\prod_{i=1}^{r} X_{i}-\pi$ and $\prod_{j=1}^{s} Y_{j}-\pi$. The morphism $f$ factors through the morphism $g: \operatorname{Spec} A \rightarrow \operatorname{Spec} \mathcal{O}_{K}\left[X_{1}, \ldots, X_{n}, Y_{1}, \ldots, Y_{n}\right] / J$ which is etale. Moreover, $J$ is actually generated by $\prod_{i=1}^{r} X_{i}-\pi$ and $\prod_{j=1}^{s} Y_{j}-\pi$, since $g$ induces an isomorphism on completed strict local rings

$$
W\left[\left[X_{1}, \ldots, X_{n}, Y_{1}, \ldots, Y_{n}\right]\right] / J \stackrel{\sim}{\rightarrow} \mathcal{O}_{r, s}
$$

This completes the proof of the lemma. 
Let $\mathcal{A}_{U}$ be the universal abelian variety over the integral model $X_{U}$. Let $\xi$ be an irreducible representation of $G$ over $\overline{\mathbb{Q}}_{l}$, for a prime number $l \neq p$. The sheaf $\mathcal{L}_{\xi}$ extends to a lisse sheaf on the integral models $X_{U_{0}}$ and $X_{U}$. Also, $a_{\xi} \in \operatorname{End}\left(\mathcal{A}_{U}^{m_{\xi}} / X_{U}\right) \otimes_{\mathbb{Z}} \mathbb{Q}$ extends as an etale morphism on $\mathcal{A}_{U}^{m_{\xi}}$ over the integral model. We have

$$
H^{j}\left(X_{U} \times_{F^{\prime}} \bar{F}_{\mathfrak{p}}^{\prime}, \mathcal{L}_{\xi}\right) \simeq a_{\xi} H^{j+m_{\xi}}\left(\mathcal{A}_{U}^{m_{\xi}} \times_{F^{\prime}} \bar{F}_{\mathfrak{p}}^{\prime}, \overline{\mathbb{Q}}_{l}\left(t_{\xi}\right)\right)
$$

and we can compute the latter via the nearby cycles $R \psi \overline{\mathbb{Q}}_{l}$ on $\mathcal{A}_{U}^{m_{\xi}}$ over the integral model of $X_{U}$. Note that $\mathcal{A}_{U}^{m_{\xi}}$ is smooth over $X_{U}$, so $\mathcal{A}_{U}^{m_{\xi}}$ is locally etale over

$X_{r, s, m}:=\operatorname{Spec} \mathcal{O}_{K}\left[X_{1}, \ldots, X_{n}, Y_{1}, \ldots Y_{n}, Z_{1}, \ldots, Z_{m}\right] /\left(\prod_{j=1}^{r} X_{i_{j}}-\pi, \prod_{j=1}^{s} Y_{i_{j}}-\pi\right)$

for some non-negative integer $m$. 


\section{Chapter 3}

\section{Sheaves of nearby cycles}

In this chapter we will start to understand the complex of nearby cycles on a scheme $X / \mathcal{O}_{K}$ which has the same geometric properties as our Iwahori level Shimura variety $X_{U}$. We work with $K / \mathbb{Q}_{p}$ be finite with ring of integers $\mathcal{O}_{K}$ which has uniformiser $\pi$ and residue field $\mathbb{F}$. Let $I_{K}=\operatorname{Gal}\left(\bar{K} / K^{\mathrm{ur}}\right) \subset G_{K}=$ $\operatorname{Gal}(\bar{K} / K)$ be the inertia subgroup of $K$. Let $\Lambda$ be either one of $\mathbb{Z} / l^{r} \mathbb{Z}, \mathbb{Z}_{l}, \mathbb{Q}_{l}$ or $\overline{\mathbb{Q}}_{l}$ for $l \neq p$ prime. Let $X / \mathcal{O}_{K}$ be a scheme such that $X$ is locally etale over

$$
X_{r, s, m}=\operatorname{Spec} \mathcal{O}_{K}\left[X_{1}, \ldots, X_{n}, Y_{1}, \ldots Y_{n}, Z_{1}, \ldots, Z_{m}\right] /\left(\prod_{j=1}^{r} X_{j}-\pi, \prod_{j=1}^{s} Y_{j}-\pi\right)
$$

Let $Y$ be the special fiber of $X$. Assume that $Y$ is a union of closed subschemes $Y_{1, j}$ with $j \in\{1, \ldots, n\}$ which are cut out locally by one equation and that this equation over $X_{r, s, m}$ corresponds to $X_{j}=0$. Similarly, assume that $Y$ is a union of closed subschemes $Y_{2, j}$ with $j \in\{1, \ldots, n\}$ which are cut out over $X_{r, s, m}$ by $Y_{j}=0$.

Let $j: X_{K} \hookrightarrow X$ be the inclusion of the generic fiber and $i: Y \hookrightarrow X$ be the inclusion of the special fiber. Let $S=\operatorname{Spec} \mathcal{O}_{K}$, with generic point $\eta$ and closed point $s$. Let $\bar{K}$ be an algebraic closure of $K$, with ring of integers $\mathcal{O}_{\bar{K}}$. 
Let $\bar{S}=\operatorname{Spec} \mathcal{O}_{\bar{K}}$, with generic point $\bar{\eta}$ and closed point $\bar{s}$. Let $\bar{X}=X \times_{S} \bar{S}$ be the base change of $X$ to $\bar{S}$, with generic fiber $\bar{j}: X_{\bar{\eta}} \hookrightarrow \bar{X}$ and special fiber $\bar{i}: X_{\bar{s}} \hookrightarrow \bar{X}$. The sheaves of nearby cycles associated to the constant sheaf $\Lambda$ on $X_{K}$ are sheaves $R^{k} \psi \Lambda$ on $X_{\bar{s}}$ defined for $k \geq 0$ as

$$
R^{k} \psi \Lambda=\bar{i}^{*} R^{k} \bar{j}_{*} \Lambda
$$

and they have continuous actions of $I_{K}$.

Proposition 3.0.8. The action of $I_{K}$ on $R^{k} \psi \Lambda$ is trivial for any $k \geq 0$.

The proof of this proposition is based on endowing $X$ with a logarithmic structure, showing that the resulting $\log$ scheme is $\log$ smooth over Spec $\mathcal{O}_{K}$ (with the canonical log structure determined by the special fiber) and then using the explicit computation of the action of $I_{K}$ on the sheaves of nearby cycles that was done by Nakayama [Na].

\subsection{Log structures}

Definition 3.1.1. A $\log$ structure on a scheme $Z$ is a sheaf of monoids $M$ together with a morphism $\alpha: M \rightarrow \mathcal{O}_{Z}$ such that $\alpha$ induces an isomorphism $\alpha^{-1}\left(\mathcal{O}_{Z}^{*}\right) \simeq \mathcal{O}_{Z}^{*}$. A scheme endowed with a log structure is a log scheme. A morphism of $\log$ schemes $\left(Z_{1}, M_{1}\right) \rightarrow\left(Z_{2}, M_{2}\right)$ consists of a pair $(f, h)$ where $f: Z_{1} \rightarrow Z_{2}$ is a morphism of schemes and $h: f^{*} M_{2} \rightarrow M_{1}$ is a morphism of sheaves of monoids.

From now on, we will regard $\mathcal{O}_{Z}^{*}$ as a subsheaf of $M$ via $\alpha^{-1}$ and define $\bar{M}:=M / \mathcal{O}_{Z}^{*}$.

Given a scheme $Z$ and a closed subscheme $V$ with complement $U$ there is a canonical way to associate to $V$ a $\log$ structure. If $j: U \hookrightarrow X$ is an open 
immersion, we can simply define $M=j_{*}\left(\left(\mathcal{O}_{X} \mid U\right)^{*}\right) \cap \mathcal{O}_{X} \rightarrow \mathcal{O}_{X}$. This amounts to formally "adjoining" the sections of $\mathcal{O}_{X}$ which are invertible outside $V$ to the units $\mathcal{O}_{X}^{*}$. The sheaf $\bar{M}$ will be supported on $V$.

If $P$ is a monoid, then the scheme Spec $\mathbb{Z}[P]$ has a canonical log structure associated to the natural map $P \rightarrow \mathbb{Z}[P]$. A chart for a $\log$ structure on $Z$ is given by a monoid $P$ and a map $Z \rightarrow$ Spec $\mathbb{Z}[P]$ such that the log structure on $Z$ is pulled back from the canonical log structure on Spec $\mathbb{Z}[P]$. A chart for a morphism of $\log$ schemes $Z_{1} \rightarrow Z_{2}$ is a triple of maps $Z_{1} \rightarrow \operatorname{Spec} \mathbb{Z}[Q]$, $Z_{2} \rightarrow$ Spec $\mathbb{Z}[P]$ and $P \rightarrow Q$ such that the first two maps are charts for the log structures on $Z_{1}$ and $Z_{2}$ and such that the obvious diagram is commutative.

For more background on log schemes, the reader should consult [I1, K].

For a scheme over $\mathcal{O}_{K}$, we let $j$ denote the open immersion of its generic fiber and $i$ the closed immersion of its special fiber into the scheme. We endow $S=\operatorname{Spec} \mathcal{O}_{K}$ with the $\log$ structure given by $N=j_{*}\left(K^{*}\right) \cap \mathcal{O}_{K} \hookrightarrow \mathcal{O}_{K}$. The sheaf $\bar{N}$ is trivial outside the closed point and is isomorphic to a copy of $\mathbb{N}$ over the closed point. Another way to describe the $\log$ structure on $S$ is by pullback of the canonical log structure via the map

$$
S \rightarrow \operatorname{Spec} \mathbb{Z}[\mathbb{N}]
$$

where $1 \mapsto \pi \in \mathcal{O}_{K}$.

We endow $X$ with the log structure given by $M=j_{*}\left(\mathcal{O}_{X_{K}}^{*}\right) \cap \mathcal{O}_{X} \hookrightarrow \mathcal{O}_{X}$. It is easy to check that the only sections of $\mathcal{O}_{X}$ which are invertible outside the special fiber, but not invertible globally are those given locally by the images of the $X_{i}$ for $1 \leq i \leq r$ and the $Y_{j}$ for $1 \leq j \leq s$. On etale neighborhoods $U$ of $X$ which are etale over $X_{r, s, m}$ this $\log$ structure is given by the chart

$$
U \rightarrow X_{r, s, m} \rightarrow \operatorname{Spec} \mathbb{Z}\left[P_{r, s}\right]
$$


where

$$
P_{r, s}:=\left(\mathbb{N}^{r} \oplus \mathbb{N}^{s}\right) /((1, \ldots 1,0, \ldots 0)=(0, \ldots 0,1, \ldots 1))
$$

The map $X_{r, s, m} \rightarrow$ Spec $\mathbb{Z}\left[P_{r, s}\right]$ can be described as follows: the element with 1 only in the $k$ th place, $(0, \ldots, 0,1,0, \ldots 0) \in P_{r, s}$ maps to $X_{k}$ if $k \leq r$ and to $Y_{k-r}$ if $k \geq r+1$. Note that the $\log$ structure on $X$ is trivial outside the special fiber, so $X$ is a vertical $\log$ scheme.

The map $X \rightarrow S$ induces a map of the corresponding log schemes. Etale locally, this map has a chart subordinate to the map of monoids $\mathbb{N} \rightarrow P_{r, s}$ such that

$$
1 \mapsto(1, \ldots, 1,0, \ldots, 0)=(0, \ldots 0,1, \ldots 1)
$$

to reflect the relations $X_{1} \ldots X_{r}=Y_{1} \ldots Y_{s}=\pi$.

Lemma 3.1.2. The map of log schemes $(X, M) \rightarrow(S, N)$ is $\log$ smooth.

Proof. The map of monoids $\mathbb{N} \rightarrow P_{r, s}$ induces a map on groups $\mathbb{Z} \rightarrow P_{r, s}^{g p}$, which is injective and has torsion-free cokernel $\mathbb{Z}^{r+s-2}$. Since the map of log schemes $(X, M) \rightarrow(S, N)$ is given etale locally by charts subordinate to such maps of monoids, by Theorem 3.5 of $[\mathrm{K}]$ the map $(X, M) \rightarrow(S, N)$ is $\log$ smooth.

\subsection{Nearby cycles and log schemes}

There is a generalization of the functor of nearby cycles to the category of log schemes.

Recall that $\mathcal{O}_{\bar{K}}$ is the integral closure of $\mathcal{O}_{K}$ in $\bar{K}$ and $\bar{S}=\operatorname{Spec} \mathcal{O}_{\bar{K}}$, with generic point $\bar{\eta}$ and closed point $\bar{s}$. The canonical log structure associated to the special fiber (given by the inclusion $\left.\bar{j}_{*}\left(\bar{K}^{*}\right) \cap \mathcal{O}_{\bar{K}} \hookrightarrow \mathcal{O}_{\bar{K}}\right)$ defines a log scheme $\tilde{S}$ with generic point $\bar{\eta}$ and closed point $\tilde{s}$. Note that $\tilde{s}$ is a log geometric point of $\tilde{S}$, so it has the same underlying scheme as $\bar{s}$. The Galois group $G_{K}$ acts on 
$\tilde{s}$ through its tame quotient. Let $\tilde{X}=X \times_{S} \tilde{S}$ in the category of log schemes, with special fiber $X_{\tilde{s}}$ and generic fiber $X_{\bar{\eta}}$. Note that, in general, the underlying scheme of $X_{\tilde{s}}$ is not the same as that of $X_{\bar{s}}$. This is because $X_{\tilde{s}}$ is the fiber product of $X_{\bar{s}}$ and $\tilde{s}$ in the category of integral and saturated log schemes and saturation corresponds to normalization, so it changes the underlying scheme.

The sheaves of $\log$ nearby cycles are sheaves on $X_{\tilde{s}}$ defined by

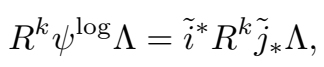

where $\tilde{i}, \tilde{j}$ are the obvious maps and the direct and inverse images are taken with respect to the Kummer etale topology. Theorem 3.2 of [Na] states that

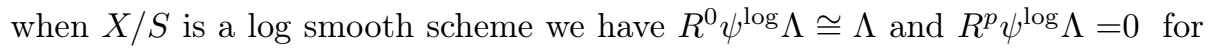
$p>0$. Let

$$
\tilde{\epsilon}: \tilde{X} \rightarrow \bar{X}
$$

which restricts to $\epsilon: X_{\bar{\eta}} \rightarrow X_{\bar{\eta}}$, be the morphism that simply forgets the log structure. Note that we have $\bar{j}_{*} \epsilon_{*}=\tilde{\epsilon}_{*} \tilde{j}_{*}$, by commutativity of the square

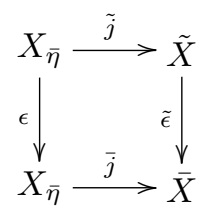

We also have $\bar{i}^{*} R \tilde{\epsilon}_{*} \mathcal{F} \simeq R \tilde{\epsilon}_{*} \tilde{i}^{*} \mathcal{F}$ for every Kummer etale sheaf $\mathcal{F}$, by strict base change (see Proposition 6.3 of [I1]). We deduce that

$$
\bar{i}^{*} \bar{j}_{*} \epsilon_{*}=\tilde{\epsilon}_{*} \tilde{i}^{*} \tilde{j}_{*}
$$

so the corresponding derived functors must satisfy a similar relation. When we write this out, using $R \psi^{\log } \Lambda \cong \Lambda$ by Nakayama's result and $R \epsilon_{*} \Lambda \cong \Lambda$ because 
the $\log$ structure is vertical and so $\epsilon$ is an isomorphism, we get

$$
R^{k} \psi^{\mathrm{cl}} \Lambda=R^{k} \tilde{\epsilon}_{*}\left(\Lambda \mid X_{\tilde{s}}\right)
$$

Therefore, it suffices to figure out what the sheaves $R^{k} \tilde{\epsilon}_{*} \Lambda$ look like and how $I_{K}$ acts on them, where $\tilde{\epsilon}: X_{\tilde{s}} \rightarrow X_{\bar{s}}$. This has been done in general by Nakayama, Theorem 3.5 of [Na], thus deriving an SGA 7 I.3.3-type formula for log smooth schemes. We will describe his argument below and specialize to our particular case.

Lemma 3.2.1. $I_{K}$ acts on $R^{p} \epsilon_{*}\left(\Lambda \mid X_{\tilde{s}}\right)$ through its tame quotient.

Proof. Let $S^{t}=\operatorname{Spec} \mathcal{O}_{K^{t}}$ endowed with the canonical log structure (here $K^{t} \subset$ $\bar{K}$ is the maximal extension of $K$ which is tamely ramified). The closed point $s^{t}$ with its induced log structure is a universal Kummer etale cover of $s$ and $I_{K}$ acts on it through its tame quotient $I^{t}$. Moreover, the projection $\tilde{s} \rightarrow s^{t}$ is a limit of universal Kummer homeomorphisms and it remains so after base change with $X$. (See Theorem 2.8 of [I1]). Thus, every automorphism of $X_{\tilde{s}}$ comes from a unique automorphism of $X_{s^{t}}$, on which $I_{K}$ acts through $I^{t}$.

Now we have the commutative diagram

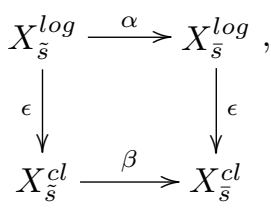

where the objects in the top row are log schemes and the objects in the bottom row are their underlying schemes. The morphisms labeled $\epsilon$ are forgetting the $\log$ structure and we have $\tilde{\epsilon}=\epsilon \circ \alpha=\beta \circ \epsilon$. We can use either of these decompositions to compute $R^{k} \tilde{\epsilon}_{*} \Lambda$. For example, we have $R \tilde{\epsilon}_{*} \Lambda=R \beta_{*} R \epsilon_{*} \Lambda$, 
which translates into having a spectral sequence

$$
R^{n-k} \beta_{*} R^{k} \epsilon_{*} \Lambda \Rightarrow R^{n} \tilde{\epsilon}_{*} \Lambda
$$

We know that $R^{k} \epsilon_{*} \Lambda=\wedge^{k} \bar{M}_{r e l}^{g p} \otimes \Lambda(-k)$, where

$$
\bar{M}_{r e l}^{g p}=\operatorname{coker}\left(\bar{N}^{g p} \rightarrow \bar{M}^{g p}\right) / \text { torsion } .
$$

Recall that $N$ is the $\log$ structure on $\mathcal{O}_{K}$ associated to its special fiber. The map of $\log$ schemes $(X, M) \rightarrow\left(\mathcal{O}_{K}, N\right)$ induces a map from the (pullback of) $N$ to $M$. We form $\bar{M}_{r e l}^{g p}$ using this map. The formula for $R^{k} \epsilon_{*} \Lambda$ follows from theorem 2.4 of $[\mathrm{KN}]$, as explained in section 3.6 of [Na]. Theorem 2.4 of $[\mathrm{KN}]$ is a statement about log schemes over $\mathbb{C}$, but the same proof also applies to the case of $\log$ schemes over a field of characteristic $p$, as explained in [I1].

On the other hand, at a geometric point $\bar{x}$ of $X_{\bar{s}}^{c l}$, we have $\left(\beta_{*} \mathcal{F}\right)_{\bar{x}} \cong \mathcal{F}\left[E_{\bar{x}}\right]$ for a sheaf $\mathcal{F}$ of $\Lambda$-modules on $X_{\tilde{s}}^{c l}$, where $E_{\bar{x}}$ is the cokernel of the map of log inertia groups

$$
I_{x} \rightarrow I_{s}
$$

Indeed, $\beta^{-1}(\bar{x})$ consists of \#coker $\left(I_{x} \rightarrow I_{s}\right)$ points, which follows from the fact that $X_{\tilde{s}}^{c l}$ is the normalization of $\left(X_{\bar{s}} \times_{\bar{s}} \tilde{s}\right)^{c l}$. The higher derived functors $R^{n-k} \beta_{*} \mathcal{F}$ are all trivial, since $\beta_{*}$ is exact. Therefore, the spectral sequence becomes

$$
\wedge^{k} \bar{M}_{r e l, \bar{x}}^{g p} \otimes \Lambda\left[E_{\bar{x}}\right] \otimes \Lambda(-k)=\left(R^{k} \tilde{\epsilon}_{*} \Lambda\right)_{\bar{x}}
$$

The tame inertia acts on the stalks of these sheaves through $I^{t} \cong I_{s} \mapsto \Lambda\left[I_{s}\right] \rightarrow$ $\Lambda\left[E_{\bar{x}}\right]$. 
In our particular case, it is easy to compute $R^{k} \tilde{\epsilon}_{*} \Lambda$ globally. Let

$$
\hat{\mathbb{Z}}^{\prime}(1)=\lim _{(m, p)=1} \mu_{m}
$$

We have

$$
I_{x}=\operatorname{Hom}\left(\bar{M}_{x}^{g p}, \hat{\mathbb{Z}}^{\prime}(1)\right)
$$

and

$$
I_{s}=\operatorname{Hom}\left(\bar{N}_{s}^{g p}, \hat{\mathbb{Z}}^{\prime}(1)\right) .
$$

The map of inertia groups is induced by the map $\bar{N}_{s}^{g p} \rightarrow \bar{M}_{x}^{g p}$, which is determined by $1 \mapsto(1, \ldots, 1,0 \ldots, 0)$, where the first $n$ terms are nonzero. Any homomorphism of $\bar{N}_{s}^{g p} \cong \mathbb{Z} \rightarrow \hat{\mathbb{Z}}^{\prime}(1)$ can be obtained from some homomorphism $\bar{M}_{x}^{g p} \rightarrow \hat{\mathbb{Z}}^{\prime}(1)$. Thus $E_{\bar{x}}$ is trivial for all log geometric points $\bar{x}$ and $I^{t}$ acts trivially on the stalks of the sheaves of nearby cycles.

Moreover, in our situation we can check that $\beta$ is an isomorphism, which follows from the fact that $X_{\bar{s}}$ is reduced, which can be checked etale locally. Indeed, if $X$ is reduced, then the underlying scheme of $X_{\tilde{s}}^{\text {log }}$ is the same as $X_{\bar{s}}$, since $X_{\tilde{s}}^{\log }$ is defined as the inverse limit over $n \in \mathbb{N}$ prime to $p$ of fiber products of fs $\log$ schemes

$$
\left(X_{\bar{s}}, M\right) \times_{(\overline{\mathbb{F}}, N), \gamma_{n}}(\overline{\mathbb{F}}, N),
$$

where $\gamma_{n}$ is the identity on the underlying schemes and is multiplication by $n$ on the non-trivial part of the log structures. The underlying scheme of a fiber product of fs log schemes is not usually the same as the fiber product of underlying schemes. The reason for this is that the log structure on the fiber product doesn't need to apriori be saturated, so we may need to introduce additional units. However, it can be checked that if $X$ is reduced then the 
product log structure is already saturated. Thus we have the global isomorphism

$$
R^{k} \tilde{\epsilon}_{*} \Lambda \simeq \wedge^{k} \bar{M}_{r e l}^{g p} \otimes \Lambda(-k)
$$

The above discussion also allows us to determine the sheaves of nearby cycles. Indeed, we have $R^{k} \psi \Lambda \simeq \wedge^{k} \bar{M}_{\text {rel }}^{g p} \otimes \Lambda(-k)$ and $\bar{M}_{\text {rel }}^{g p}$ can be computed explicitly on neighborhoods. If $U$ is a neighborhood of $X$ with $U$ etale over $X_{r, s}$ then the $\log$ structure on $U$ is induced from the $\log$ structure on $X_{r, s}$. Let $J_{1}, J_{2} \subseteq$ $\{1, \ldots, n\}$ be sets of indices with cardinalities $r$ and $s$ respectively, corresponding to sets of divisors $Y_{1, i}$ and $Y_{2, j}$ which intersect $U$.

Proposition 3.2.2. For $i=1,2$ and $j=1, \ldots, n$, let $a_{j}^{i}: Y_{i, j} \hookrightarrow Y$ denote the closed immersion. Then we have the following isomorphism of sheaves on $U$ :

$$
\left.\left.R^{k} \psi \Lambda(k)\right|_{U} \simeq \wedge^{k}\left[\left(\left(\oplus_{j \in J_{1}} a_{j *}^{1} \Lambda\right) / \Lambda\right) \oplus\left(\left(\oplus_{j \in J_{2}} a_{j *}^{2} \Lambda\right) / \Lambda\right)\right]\right|_{U},
$$

where for $i=1,2$ we are quotienting by the canonical diagonal map

$$
\Lambda \rightarrow \oplus_{j \in J_{i}} a_{j *}^{i} \Lambda
$$

Proof. This follows from the fact that on $U$ a chart for the $\log$ structure $\left.M\right|_{U}$ can be given by the map

$$
U \rightarrow X_{r, s, m} \rightarrow \operatorname{Spec} \mathbb{Z}\left[P_{r, s}\right]
$$

as explained in 3.1 , so that $\left.\bar{M}_{r e l}^{g p}\right|_{U}$ can be identified with $\left(\left(\oplus_{j \in J_{1}} a_{j *}^{1} \mathbb{Z}\right) / \mathbb{Z}\right) \oplus$ $\left.\left(\left(\oplus_{j \in J_{2}} a_{j *}^{2} \mathbb{Z}\right) / \mathbb{Z}\right)\right|_{U}$ 
We can now define a global map of sheaves

$$
\wedge^{k}\left[\left(\oplus_{j=1}^{n} a_{j *}^{1} \Lambda\right) \oplus\left(\oplus_{j=1}^{n} a_{j *}^{2} \Lambda\right)\right] \rightarrow \wedge^{k} \bar{M}_{r e l}^{g p} \otimes_{\mathbb{Z}} \Lambda \simeq R^{k} \psi \Lambda(k)
$$

It is enough to describe a global map of sheaves $a_{j *}^{i} \mathbb{Z} \rightarrow \bar{M}_{r e l}^{g p}$. Locally, on neighborhoods $U$, we map section $1 \in a_{j *}^{i} \mathbb{Z}(U)$ to the image in $\bar{M}_{r e l}^{g p}(U)$ of a generator for the local equation defining $Y_{i, j}$ (this is independent of the choice of generator). These local maps over neighborhoods $U$ glue to give a global map, since the two images of 1 in $M\left(U \times_{X} U^{\prime}\right)$ differ by units, so they are identified once we pass to $\bar{M}_{r e l}^{g p}\left(U \times_{X} U^{\prime}\right)$. We see from the local description in proposition 3.2.2 that the above map of sheaves is surjective and that the kernel is generated by images of the two diagonal maps $\Lambda \rightarrow \oplus_{j=1}^{n} a_{j *}^{i} \Lambda$ for $i=1,2$.

Corollary 3.2.3. There is a global isomorphism

$$
\wedge^{k}\left[\left(\left(\oplus_{j \in J_{1}} a_{j *}^{1} \Lambda\right) / \Lambda\right) \oplus\left(\left(\oplus_{j \in J_{2}} a_{j *}^{2} \Lambda\right) / \Lambda\right)\right] \simeq R^{k} \psi \Lambda(k)
$$

Let $\mathcal{L}_{1}=\left(\oplus_{j=1}^{n} a_{j *}^{1} \Lambda\right) / \Lambda$ and $\mathcal{L}_{2}=\left(\oplus_{j=1}^{n} a_{j *}^{2} \Lambda\right) / \Lambda$. From the above corollary, we see that $R^{k} \psi \Lambda(k)$ can be decomposed as $\sum_{l=0}^{k} \wedge^{l} \mathcal{L}_{1} \otimes \wedge^{k-l} \mathcal{L}_{2}$. If $X$ was actually a product of strictly semistable schemes, $X=X_{1} \times_{S} X_{2}$, then the sheaves $\wedge^{l} \mathcal{L}_{1}$ and $\wedge^{k-l} \mathcal{L}_{2}$ would have an interpretation as pullbacks of the nearby cycles sheaves $R^{l} \psi \Lambda$ and $R^{k-l} \psi \Lambda$ associated to $X_{1}$ and $X_{2}$ respectively. Corollary 3.2.3 would then look like a Künneth-type formula computing the sheaves of nearby cycles for a product of strictly semistable schemes. In fact, in such a situation, the computation of the sheaves of nearby cycles reflects the stronger relation between the actual complexes of nearby cycles

$$
R \psi \Lambda_{X_{1} \times_{S} X_{2}} \simeq R \psi \Lambda_{X_{1}} \otimes_{\bar{s}}^{L} R \psi \Lambda_{X_{2}}
$$


which takes place in the derived category of constructible sheaves of $\Lambda$-modules on $\left(X_{1} \times_{S} X_{2}\right)_{\bar{s}}$. This result was proven in [I2] for a product of schemes of finite type. The isomorphism is stated in the case when $\Lambda$ is torsion, however the analogue morphism for $\Lambda$ a finite extension of $\mathbb{Z}_{l}$ or $\mathbb{Q}_{l}$ can be defined by passage to the limit (see the formalism in [E]) and it will still be an isomorphism. We would like to give here a different proof of this result in the case of the product of two strictly semistable schemes. We will use log schemes, specifically Nakayama's computation of log vanishing cycles for log smooth schemes.

Recall that the scheme $S$ has generic point $\eta$ and closed point $s$. We will freely use the notations $\bar{S}, \tilde{S}$ and $\bar{s}, \tilde{s}$, and also the corresponding notations for a scheme $X$ fixed in the begining of this subsection. We first need a preliminary result.

Lemma 3.2.4. Let $X_{1}$ be a strictly semistable scheme over $S$. Then the sheaves $R^{k} \psi \Lambda$ are flat over $\Lambda$.

Proof. By Proposition 1.1.2.1 of [Sa2], we have an exact sequence of sheaves on $X_{1, \bar{s}}$

$$
0 \rightarrow R^{k} \psi \Lambda \rightarrow i^{*} R^{k+1} j_{*} \Lambda(1) \rightarrow R^{k+1} \psi \Lambda(1) \rightarrow 0
$$

We will prove by induction on $k$ that $R^{n-k} \psi \Lambda$ is flat over $\Lambda$. Indeed, $R^{n} \psi \Lambda=0$ so the induction hypothesis is true for $k=0$. For the induction step, note that we can compute $i^{*} R^{n-k+1} j_{*} \Lambda$ using log etale cohomology. Since $X_{1}$ is strictly semistable, it can be endowed with the canonical log structure $M_{1}$ associated to the special fiber. If $a_{i}^{1}: Y_{1, i} \hookrightarrow Y$ are the closed embeddings, then we have

$$
\bar{M}_{1}^{\mathrm{gp}}=\left(M_{1} / \mathcal{O}_{X_{1}}^{*}\right)^{\mathrm{gp}} \simeq \oplus_{i=1}^{n} a_{i *}^{1} \mathbb{Z}
$$

By Theorem 0.2 (purity for log smooth morphisms) and Proposition 2.0.2 of [Na], we can compute $i^{*} R^{n-k+1} j_{*} \Lambda$ in the same way we have computed $R^{k} \psi \Lambda$ 
above, getting:

$$
i^{*} R^{n-k+1} j_{*} \Lambda \simeq \wedge^{n-k+1}\left(\bar{M}_{1}^{\mathrm{gp}}\right) \otimes_{\mathbb{Z}} \Lambda(-n+k-1),
$$

which is flat over $\Lambda$ by 3.1. In the short exact sequence

$$
0 \rightarrow R^{n-k} \psi \Lambda \rightarrow i^{*} R^{n-k+1} j_{*} \Lambda(1) \rightarrow R^{n-k+1} \psi \Lambda(1) \rightarrow 0
$$

the middle term is flat, the right term is flat by the induction hypothesis, so the left term must be flat as well.

Proposition 3.2.5. Let $X_{1}$ and $X_{2}$ be strictly semistable schemes over $S$. Then we have the following equality in the derived category of constructible $\Lambda\left[I_{s}\right]$ modules on $\left(X_{1} \times{ }_{S} X_{2}\right)_{s}$ :

$$
R \psi\left(\Lambda_{X_{1, \eta}}\right) \otimes_{s}^{L} R \psi\left(\Lambda_{X_{2, \eta}}\right) \simeq R \psi\left(\Lambda_{\left(X_{1} \times_{S} X_{2}\right)_{\eta}}\right)
$$

where the external tensor product of a complexes is obtained by taking $p_{1}^{*} \otimes p r_{2}^{*}$ and where the superscript $L$ refers to left derived tensor product.

Proof. We've seen from the above discussion that in the case of a log smooth scheme with vertical log structure the complex of vanishing cycles depends only on the special fiber endowed with the canonical log structure. In other words, for $i=1,2$, we have $R \psi \Lambda_{X_{i, \eta}} \simeq R \tilde{\epsilon}_{i, *} \Lambda_{X_{i, s}}$ as complexes on $X_{i, s}$, where $\tilde{\epsilon}_{i}$ : $\tilde{X}_{i, \tilde{s}} \rightarrow \bar{X}_{i, \bar{s}}$ is the identity morphism on the underlying schemes and forgets the $\log$ structure. Analogously, we also have $R \psi \Lambda_{\left(X_{1} \times{ }_{S} X_{2}\right)_{\eta}}=R \tilde{\epsilon}_{*} \Lambda$, where

$$
\tilde{\epsilon}:\left(\tilde{X}_{1} \times_{\tilde{S}} \tilde{X}_{2}\right)_{\tilde{s}} \rightarrow\left(\bar{X}_{1} \times_{\bar{S}} \bar{X}_{2}\right)_{\bar{s}}
$$

is the morphism which forgets the log structure. (Here we've used the fact that 
the fiber product of log smooth schemes with vertical log structure is log smooth with vertical log structure and that the underlying scheme of the fiber product of $\log$ schemes $\tilde{X}_{1} \times_{\tilde{S}} \tilde{X}_{2}$ is just $\bar{X}_{1} \times_{\bar{S}} \bar{X}_{2}$; the latter holds since the induced $\log$ structure on $\bar{X}_{1} \times_{\bar{S}} \bar{X}_{2}$ is saturated.) Therefore, it suffices to prove that we have an isomorphism

$$
R \tilde{\epsilon}_{*} \Lambda_{\left(\tilde{X}_{1} \times_{\tilde{S}} \tilde{X}_{2}\right)_{\tilde{s}}} \simeq R \tilde{\epsilon}_{1, *} \Lambda_{\tilde{X}_{1, \tilde{s}}} \otimes_{\tilde{s}}^{L} R \tilde{\epsilon}_{2, *} \Lambda_{\tilde{X}_{2, \tilde{s}}}
$$

in the derived category of constructible sheaves of $\Lambda\left[I_{s}\right]$-modules on $\left(\bar{X}_{1} \times{ }_{\bar{S}} \bar{X}_{2}\right)_{\bar{s}}$.

It is enough to show that the Künneth map

$$
\mathcal{C}=R \tilde{\epsilon}_{1, *} \Lambda_{\tilde{X}_{1, s}} \otimes_{\tilde{s}}^{L} R \tilde{\epsilon}_{2, *} \Lambda_{\tilde{X}_{2, \tilde{s}}} \rightarrow R \tilde{\epsilon}_{*} \Lambda_{\left(\tilde{X}_{1} \times \tilde{S}_{\tilde{S}} \tilde{X}_{\tilde{s}}\right.}=\mathcal{D}
$$

which is defined as in [SGA4] XVII 5.4.1.4, induces an isomorphism on the cohomology of the two complexes above, for then the map itself will be a quasiisomorphism. The cohomology of the product complex can be computed using a Künneth formula as $H^{n}(\mathcal{C})=\bigoplus_{k=0}^{n} R^{k} \tilde{\epsilon}_{1, *} \Lambda \otimes_{\bar{s}} R^{n-k} \tilde{\epsilon}_{2, *} \Lambda$. In general, the Künneth formula involves a spectral sequence with terms

$$
E_{2}^{l, n-l}=\sum_{k=0}^{n-l} \operatorname{Tor}_{l}^{\Lambda\left[I_{s}\right]}\left(R^{k} \tilde{\epsilon}_{1, *} \Lambda, R^{n-l-k} \tilde{\epsilon}_{2, *} \Lambda\right) \Rightarrow H^{n}(\mathcal{C}),
$$

see [EGA3] XVII 6.5.4.2 for a statement using homology. In our case the cohomology sheaves $R^{k} \tilde{\epsilon}_{i, *} \Lambda$ are flat $\Lambda$-modules with trivial $I_{s}$-action by Lemmas 3.0.8 and 3.2.4, so for $l>0$ all the $E_{2}^{l, n-l}$ terms vanish. (Alternatively, one can prove the formula for $H^{n}(\mathcal{C})$ by taking flat resolutions for both of the factor complexes and using the fact that the cohomology sheaves of the flat complexes are flat as well.)

In order to prove that the induced map $H^{n}(\mathcal{C}) \rightarrow H^{n}(\mathcal{D})$ is an isomorphism, 
it suffices to check that it induces an isomorphism on stalks at geometric points. Let $x$ be a geometric point of $X_{1} \times_{S} X_{2}$ above the geometric point $\bar{s}$ of $S$. The point $x$ will project to geometric points $x_{1}$ and $x_{2}$ of $X_{1}$ and $X_{2}$. From [I1] it follows that there is an isomorphism on stalks

$$
R^{k} \tilde{\epsilon}_{i, *} \Lambda_{x_{i}} \simeq H^{k}\left(J_{i}, \Lambda\right)
$$

for $0 \leq k \leq n$ and $i=1,2$, where $J_{i}$ is the relative log inertia group

$$
\operatorname{ker}\left(\pi_{1}^{\log }\left(X_{i}, x_{i}\right) \rightarrow \pi_{1}^{\log }(S, s)\right)
$$

A similar statement holds for the stalks at $x$

$$
R^{n} \tilde{\epsilon}_{*} \Lambda_{x} \simeq H^{n}(J, \Lambda)
$$

where $J$ is the relative $\log$ inertia group $\operatorname{ker}\left(\pi_{1}^{\log }(X, x) \rightarrow \pi_{1}^{\log }(S, s)\right)$. Directly from the definition of the $\log$ fundamental group we can compute $J=J_{1} \times J_{2}$. We have the following commutative diagram

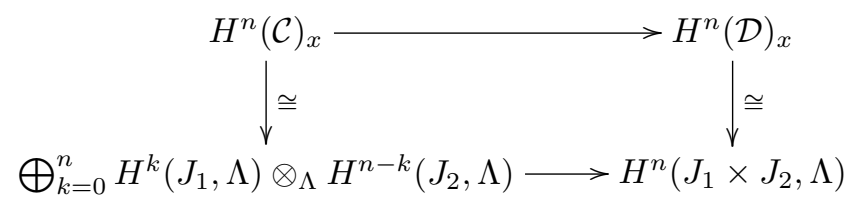

where the bottom arrow is the Künneth map in group cohomology and is also an isomorphism. (Again, the Künneth spectral sequence

$$
E_{2}^{l, n-l}=\sum_{k=0}^{n-l} \operatorname{Tor}_{l}^{\Lambda}\left(H^{k}\left(J_{1}, \Lambda\right), H^{n-k}\left(J_{2}, \Lambda\right)\right)
$$

degenerates at $E_{2}$ and all terms outside the vertical line $l=0$ vanish be- 
cause these cohomology groups are flat $\Lambda$-modules.) Therefore the top arrow $H^{n}(\mathcal{C})_{x} \rightarrow H^{n}(\mathcal{D})_{x}$ has to be an isomorphism for all geometric points $x$ of $X$ which means it comes from a global isomorphism of sheaves on $X$. 


\section{Chapter 4}

\section{The monodromy filtration}

\subsection{Overview of the strictly semistable case}

In this section, we will explain a way of writing down explicitly the monodromy filtration on the complex of nearby cycles $R \psi \Lambda$, in the case of a strictly semistable scheme. Our exposition will follow that of [Sa2], which constructs the monodromy filtration using perverse sheaves. We let $\Lambda=\mathbb{Z} / l^{r} \mathbb{Z}, \mathbb{Z}_{l}, \mathbb{Q}_{l}$ or $\overline{\mathbb{Q}}_{l}$. In fact, the proofs use $\Lambda=\mathbb{Z} / l^{r} \mathbb{Z}$, then the results extend to $\Lambda=\mathbb{Z}_{l}, \mathbb{Q}_{l}, \overline{\mathbb{Q}}_{l}$.

Let $X_{1} / \mathcal{O}_{K}$ be a strictly semistable scheme of relative dimension $n-1$ with generic fiber $X_{1, \eta}$ and special fiber $Y_{1}=X_{1, s}$. Let $R \psi \Lambda=\bar{i}^{*} R \bar{j}_{*} \Lambda$ be the complex of nearby cycles over $Y_{1, \overline{\mathbb{F}}}$. Let $D_{1}, \ldots, D_{m}$ be the irreducible components of $Y_{1}$ and for each index set $I \subseteq\{1, \ldots, m\}$ let $Y_{I}=\cap_{i \in I} D_{i}$ and $a_{I}: Y_{I} \rightarrow Y_{1}$ be the immersion. The scheme $Y_{I}$ is smooth of dimension $n-1-k$ if $\# I=k+1$. For all $0 \leq k \leq m-1$ we set

$$
Y_{1}^{(k)}=\bigsqcup_{I \subseteq\{1, \ldots, m\}, \# I=k+1} Y_{I}
$$


and let $a_{k}: Y_{1}^{(k)} \rightarrow Y_{1}$ be the projection. We identify $a_{k *} \Lambda=\wedge^{k+1} a_{0 *} \Lambda$.

We will work in the derived category of bounded complexes of constructible sheaves of $\Lambda$-modules on $Y_{1, \overline{\mathbb{F}}}$. We will denote this category by $D_{c}^{b}\left(Y_{1, \overline{\mathbb{F}}}, \Lambda\right)$.

Let $\partial[\pi]$ be the boundary of $\pi$ with respect to the Kummer sequence obtained by applying $i^{*} R j_{*}$ to the exact sequence of etale sheaves on $X_{1, \eta}$

$$
0 \rightarrow \Lambda(1) \rightarrow \mathcal{O}_{X_{1, \eta}}^{*} \rightarrow \mathcal{O}_{X_{1, \eta}}^{*} \rightarrow 0
$$

for $\Lambda=\mathbb{Z} / l^{r} \mathbb{Z}$. Taking an inverse limit over $r$ and tensoring we get an element $\partial[\pi] \in i^{*} R^{1} j_{*} \Lambda(1)$ for $\Lambda=\mathbb{Q}_{l}$ or $\overline{\mathbb{Q}}_{l}$. Let $\theta: \Lambda_{Y_{1}} \rightarrow i^{*} R^{1} j_{*} \Lambda(1)$ be the map sending 1 to $\partial[\pi]$. Let $\delta: \Lambda_{Y_{1}} \rightarrow a_{0 *} \Lambda$ be the canonical map. The following result appears as Corollary 1.3 of [Sa2].

Proposition 4.1.1. 1. There is an isomorphism of exact sequences

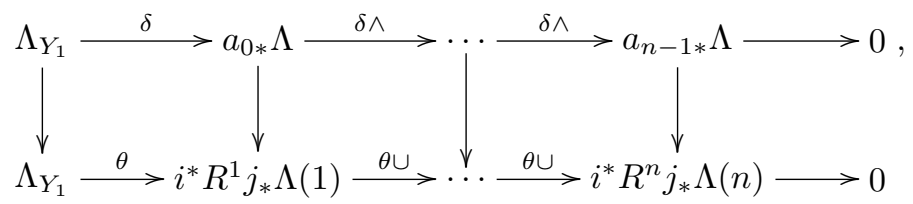

where the first vertical arrow is the identity and all the other vertical arrows are isomorphisms.

2. For $k \geq 0$ we have an exact sequence

$$
0 \rightarrow R^{k} \psi \Lambda \rightarrow i^{*} R^{k+1} j_{*} \Lambda(1) \rightarrow \cdots \rightarrow i^{*} R^{n} j_{*} \Lambda(n-k) \rightarrow 0,
$$

where all the horizontal maps are induced from $\theta \cup$.

Note. 1. The vertical isomorphisms in the first part of Proposition 4.1.1 come from the Kummer sequence corresponding to each of the $D_{i}$ for $i=1, \ldots, m$. The maps $\theta_{i}: \Lambda_{D_{i}} \rightarrow i^{*} R^{1} j_{*} \Lambda(1)$ are defined by sending 1 to $\partial\left[\pi_{i}\right]$, where $\pi_{i}$ is the generator of the ideal defining $D_{i}$ and $\partial$ is the connecting differential in the 
Kummer sequence. The isomorphism $a_{0 *} \Lambda \stackrel{\sim}{\rightarrow} i^{*} R^{1} j_{*} \Lambda(1)$ is the direct sum of the $\theta_{i}$ for $i=1, \ldots, m$.

2. Putting together the two isomorphisms, we get a quasi-isomorphism of complexes

$$
R^{k} \psi \Lambda(k)[-k] \stackrel{\sim}{\rightarrow}\left[a_{k *} \Lambda \rightarrow \cdots \rightarrow a_{n-1 *} \Lambda \rightarrow 0\right],
$$

where $R^{k} \psi \Lambda(k)$ is put in degree $k$ and $a_{n-1 *} \Lambda$ is put in degree $n-1$.

Lemma 4.1.2. The complex $a_{l *} \Lambda[-l]$ is a-(n-1)-shifted perverse sheaf for all $0 \leq l \leq n-1$ and so is the complex $R^{k} \psi \Lambda(k)[-k]$ for all $0 \leq k \leq n-1$.

Proof. Since $Y_{1}^{(l)}$ is smooth of dimension $n-1-l$, we know that $\Lambda[-l]$ is a $-(n-1)$-shifted perverse sheaf on $Y_{1}^{(l)}$. The map $a_{l}: Y^{(l)} \rightarrow Y$ is finite and since the direct image for a finite map is exact for the perverse $t$-structure, we deduce that $a_{l *} \Lambda[-l]$ is a $-(n-1)$-shifted perverse sheaf on $Y$. This is true for each $0 \leq l \leq n-1$. The complex $R^{k} \psi \Lambda(k)[-k]$ is a successive extension of terms of the form $a_{l *} \Lambda[-l]$ (as objects in the triangulated category $D_{c}^{b}\left(Y_{\overline{\mathbb{F}}}, \Lambda\right)$. Because the category of $-(n-1)$-shifted perverse sheaves is stable under extensions, we conclude that $R^{k} \psi \Lambda(k)[-k]$ is also a $-(n-1)$-shited perverse sheaf.

Assume $\Lambda=\mathbb{Z} / l^{r} \mathbb{Z}$. Let $\mathcal{L} \in D_{c}^{b}\left(Y_{1, \overline{\mathrm{F}}}, \Lambda\right)$ be represented by the complex

$$
\cdots \rightarrow \mathcal{L}^{k-1} \rightarrow \mathcal{L}^{k} \rightarrow \mathcal{L}^{k+1} \rightarrow \ldots
$$

Definition 4.1.3. We define $\tau_{\leq k} \mathcal{L}$ to be the standard truncation of $\mathcal{L}$, represented by the complex

$$
\cdots \rightarrow \mathcal{L}^{k-1} \rightarrow \operatorname{ker}\left(\mathcal{L}^{k} \rightarrow \mathcal{L}^{k+1}\right) \rightarrow 0
$$

Then $\tau_{\leq k}$ is a functor on $D_{c}^{b}\left(Y_{1, \bar{F}}, \Lambda\right)$. We also define $\tilde{\tau}_{\leq k} \mathcal{K}$ to be represented by 
the complex

$$
\cdots \rightarrow \mathcal{L}^{k-1} \rightarrow \mathcal{L}^{k} \rightarrow \operatorname{im}\left(\mathcal{L}^{k} \rightarrow \mathcal{L}^{k+1}\right) \rightarrow 0
$$

For every $k$ we have a quasi-isomorphism $\tau_{\leq k} \mathcal{L} \stackrel{\sim}{\rightarrow} \tilde{\tau}_{\leq k} \mathcal{L}$, which is given degree by degree by the inclusion map.

Corollary 4.1.4. The complex $R \psi \Lambda$ is a-(n-1)-shifted perverse sheaf and the truncations $\tau_{\leq k} R \psi \Lambda$ make up a decreasing filtration of $R \psi \Lambda$ by $-(n-1)$-shifted perverse sheaves.

Proof. Since the cohomology of $R \psi \Lambda$ vanishes in degrees greater than $n-1$, we have $R \psi \Lambda \simeq \tau_{\leq n-1} R \psi \Lambda$ so it suffices to prove by induction that each $\tau_{\leq k} R \psi \Lambda$ is a $-(n-1)$-shifted perverse sheaf. For $k=0$, we have $\tau_{\leq 0} R \psi \Lambda \simeq R^{0} \psi \Lambda$, which is a $-(n-1)$-shifted perverse sheaf by Lemma 4.1.2. For $k \geq 1$ we have a distinguished triangle

$$
\left(\tau_{\leq k-1} R \psi \Lambda, \tau_{\leq k} R \psi \Lambda, R^{k} \psi \Lambda[-k]\right)
$$

and assuming that $\tau_{\leq k-1} R \psi \Lambda$ is a $-(n-1)$-shifted perverse sheaf, we conclude that $\tau_{\leq k} R \psi \Lambda$ is as well. The distinguished triangles become short exact sequences in the abelian category of perverse sheaves, from which we deduce that the $\tau_{\leq k} R \psi \Lambda$ make up a decreasing filtration of $R \psi \Lambda$ and that the graded pieces of this filtration are the $R^{k} \psi \Lambda[-k]$.

Note 4.1.5. For $\Lambda=\mathbb{Z}_{l}, \mathbb{Q}_{l}$ or $\overline{\mathbb{Q}}_{l}$, we still have standard truncation functors $\tau_{\leq k}$ which give us a distinguished triangle

$$
\left(\tau_{\leq k-1} R \psi \Lambda, \tau_{\leq k} R \psi \Lambda, R^{k} \psi \Lambda[-k]\right)
$$

but the $\tau_{\leq k}$ are defined differently. With the new definition, the proof and results of Corollary 4.1.4 still go through for $\Lambda=\mathbb{Q}_{l}$ or $\overline{\mathbb{Q}}_{l}$. 
The complex $R \psi \Lambda$ has an action of $I_{s}$, which acts trivially on the cohomology sheaves $R^{k} \psi \Lambda$. From this, it follows that the action of $I_{s}$ factors through the action of its tame pro-l-quotient. Let $T$ be a generator of pro- $l$-part of the tame inertia (i.e. such that $t_{l}(T)$ is a generator of $\mathbb{Z}_{l}(1)$, where $t_{l}: I_{l} \rightarrow \mathbb{Z}_{l}(1)$ is the tame inertial character). We are interested in understanding the action of $T$ on $R \psi \Lambda$. For $\Lambda=\mathbb{Q}_{l}$ or $\overline{\mathbb{Q}}_{l}$, we're interested in understanding the action of $N=\log T$, by recovering its monodromy filtration (convolution of the kernel and image filtrations). However, the monodromy filtration of $N$ is the same as the monodromy filtration of $\nu:=T-1$ so we will explain how to compute the latter.

We've seen that $T$ acts trivially on the $R^{k} \psi \Lambda$, which means that $\nu$ sends $\tau_{\leq k} R \psi \Lambda \rightarrow \tilde{\tau}_{\leq k-1} R \psi \Lambda \stackrel{\sim}{\rightarrow} \tau_{\leq k-1} R \psi \Lambda$. We get an induced map

$$
\bar{\nu}: R^{k} \psi \Lambda[-k] \rightarrow R^{k-1} \psi \Lambda[-k+1] .
$$

We record part 4 of Lemma 2.5 of [Sa2].

Lemma 4.1.6. The map $\bar{\nu}$ and the isomorphisms of Note 4.2 make a commutative diagram

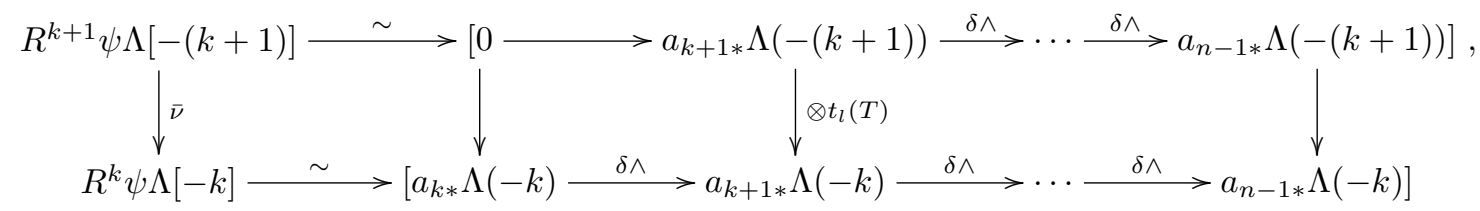

where the sheaves $a_{n-1 *} \Lambda(-(k+1))$ and $a_{n-1 *} \Lambda(-k)$ are put in degree $n-1$.

Note 4.1.7. When $\Lambda=\mathbb{Q}_{l}$ or $\overline{\mathbb{Q}}_{l}$ the monodromy operator $N=\log T$ is defined 
and it induces a map

$$
\bar{N}: R^{k} \psi \Lambda[-k] \rightarrow R^{k-1} \psi \Lambda[-k+1] .
$$

This map coincides with $\bar{\nu}$, since $\log T \equiv T-1\left(\bmod (T-1)^{2}\right)$ and $(T-1)^{2}$ sends $\tau_{\leq k} R \psi \Lambda \rightarrow \tau_{\leq k-2} R \psi \Lambda$.

From the above commutative diagram, it is easy to see that the map $\bar{\nu}$ is injective, since we can just compute the cone of the map of complexes on the right. In general, to compute the kernel and cokernel of a map of perverse sheaves, we have to compute the cone $C$ of that map, then the perverse truncation $\tau_{\geq 0}^{p} C$ will be the cokernel and $\tau_{\leq-1}^{p} C[-1]$ will be the kernel (see the proof of Theorem 1.3.6 of [BBD]). It is straightforward to check that the cone of $\bar{\nu}$ is quasi-isomorphic to $a_{k *} \Lambda(-k)[-k]$, which is a $-(n-1)$-shifted perverse sheaf. We deduce that $\bar{\nu}$ has kernel 0 and cokernel $a_{k *} \Lambda(-k)[-k]$.

The fact that $\bar{\nu}$ is injective means that the canonical filtration $\tau_{\leq k} R \psi \Lambda$ coincides with the kernel filtration of $\nu$ on $R \psi \Lambda$ and that the $R^{k} \psi \Lambda[-k]$ for $0 \leq k \leq n-1$ are the graded pieces of the kernel filtration. Moreover, the graded pieces of the induced image filtration of $\nu$ on the $R^{k} \psi \Lambda$ are $a_{k+h *} \Lambda(-h)[-(k+h)]$ for $0 \leq h \leq n-1-k$. This information suffices to reconstruct the graded pieces of the monodromy filtration on $R \psi \Lambda$.

Proposition 4.1.8. There is an isomorphism

$$
\bigoplus_{h-k=r} a_{(k+h) *} \Lambda(-h)[-(k+h)] \rightarrow G r_{r}^{M} R \psi \Lambda .
$$

This isomorphism, together with the spectral sequence associated to the monodromy filtration induces the weight spectral sequence (see Corollary 2.2.4 of $[\mathrm{Sa} 2])$. 


\subsection{The product of strictly semistable schemes}

Let $X_{1}$ and $X_{2}$ be strictly semistable schemes of relative dimension $n-1$ over $\mathcal{O}_{K}$, and let $\Lambda=\mathbb{Z} / l^{r} \mathbb{Z}, \mathbb{Z}_{l}, \mathbb{Q}_{l}$ or $\overline{\mathbb{Q}}_{l}$ (we will be more specific about $\Lambda$ where it is important). Let $R \psi \Lambda_{X_{i}}$ be the complex of nearby cycles on $X_{i, \bar{s}}$ for $i=1,2$ and let $R \psi \Lambda_{X_{1} \times X_{2}}$ be the complex of nearby cycles on $\left(X_{1} \times_{S} X_{2}\right)_{\bar{s}}$. By Proposition 3.2 .5 , we have

$$
R \psi \Lambda_{X_{1} \times X_{2}} \simeq R \psi \Lambda_{X_{1}} \otimes_{\Lambda} R \psi \Lambda_{X_{2}}
$$

and notice that this isomorphism is compatible with the action of the inertia $I$ in $G_{K}$. From Proposition 3.0.8, the action of $I$ is trivial on the cohomology sheaves of $R \psi \Lambda_{X_{1} \times X_{2}}$, so only the pro-l part of $I$ acts nontrivially on $R \psi \Lambda_{X_{1} \times X_{2}}$. Let $T$ be a generator of the pro- $l$ part of $I$ and set $\nu=T-1$. Let $\nu, \nu_{1}, \nu_{2}$ denote the action of $\nu$ on $R \psi \Lambda_{X_{1} \times X_{2}}, R \psi \Lambda_{X_{1}}$ and $R \psi \Lambda_{X_{2}}$ respectively. Since the above isomorphism is compatible with the action of $T$, we deduce that $T$ acts on $R \psi \Lambda_{X_{1}} \otimes_{\Lambda[I]} R \psi \Lambda_{X_{2}}$ via $T \otimes T$. From this, we conclude that $\nu$ acts on $R \psi \Lambda_{X_{1}} \otimes_{\Lambda[I]} R \psi \Lambda_{X_{2}}$ as $\nu_{1} \otimes 1+1 \otimes \nu_{2}+\nu_{1} \otimes \nu_{2}$

As in the proof of Proposition 3.2.2, we have a decomposition

$$
R^{k} \psi \Lambda \simeq \bigoplus_{l=0}^{k} R^{l} \psi \Lambda_{X_{1}} \otimes R^{k-l} \psi \Lambda_{X_{2}}
$$

We shall see that $\nu$ induces a map

$$
\bar{\nu}: R^{k} \psi \Lambda_{X_{1} \times X_{2}}[-k] \rightarrow R^{k-1} \psi \Lambda_{X_{1} \times X_{2}}[-k+1]
$$

which acts on $R^{l} \psi \Lambda_{X_{1}} \otimes_{\Lambda} R^{k-l} \psi \Lambda_{X_{2}}[-k]$ by $\bar{\nu}_{1} \otimes 1+1 \otimes \bar{\nu}_{2}$. First we prove a few preliminary results.

For $i=1,2$ and $0 \leq l \leq n$ define the following schemes:

- Let $Y_{i} / \mathbb{F}$ be the special fiber of $X_{i}$ 
- Let $D_{i, 1}, \ldots, D_{i, m_{i}}$ be the irreducible components of $X_{i}$

- For $J \subseteq\left\{1, \ldots, m_{i}\right\}$ let $Y_{i, J}$ be $\cap_{j \in J} D_{i, j}$ and let $a_{J}^{i}: Y_{i, J} \rightarrow Y_{i}$ be the immersion. Note that if the cardinality of $J$ is $h+1$, then the scheme $Y_{i, J}$ is smooth of dimension $n-h-1$.

- For all $0 \leq h \leq m_{i}-1$ set $Y_{i}^{(h)}=\bigsqcup_{\# J=h+1} Y_{i, J}$ and let $a_{l}^{i}: Y_{i}^{(h)} \rightarrow Y_{i}$ be the projection.

Then for each $i=1,2$ we have a resolution of $R^{h} \psi \Lambda_{X_{i}}[-h]$ in terms of the sheaves $a_{j *}^{i} \Lambda$ :

$$
R^{h} \psi \Lambda_{X_{i}}[-h] \stackrel{\sim}{\rightarrow}\left[a_{l *}^{i} \Lambda(-h) \rightarrow \cdots \rightarrow a_{n-1 *}^{i} \Lambda(-h)\right],
$$

where $a_{n-1 *}^{i} \Lambda(-h)$ is put in degree $n-1$.

Now let $Y / \mathbb{F}$ be the special fiber of $X_{1} \times X_{2}$. Let

$$
Y_{J_{1}, J_{2}}=\bigcap_{j_{1} \in J_{1}, j_{2} \in J_{2}}\left(D_{j_{1}} \times_{\mathbb{F}} D_{j_{2}}\right) .
$$

Set $Y^{\left(h_{1}, h_{2}\right)}=\bigsqcup_{\# J_{1}=h_{1}+1, \# J_{2}=h_{2}+1} Y_{J_{1}, J_{2}}$ and let $a_{h_{1}, h_{2}}: Y^{\left(h_{1}, h_{2}\right)} \rightarrow Y$ be the projection. The scheme $Y^{\left(h_{1}, h_{2}\right)}$ is smooth of dimension $2 n-2-h_{1}-h_{2}$. Note that $Y^{\left(h_{1}, h_{2}\right)}=Y_{1}^{\left(h_{1}\right)} \times Y_{2}^{\left(h_{2}\right)}$ and that $a_{h_{1}, h_{2} *} \Lambda \simeq a_{h_{1} *}^{1} \Lambda \otimes a_{h_{2} *}^{2} \Lambda$, where the tensor product of sheaves is an external tensor product.

Lemma 4.2.1. We have the following resolution of $R^{h} \psi \Lambda_{X_{1}} \otimes R^{k-h} \psi \Lambda_{X_{2}}[-k]$ as the complex

$a_{h, k-h *} \Lambda(-k) \rightarrow a_{h, k-h+1 *} \Lambda(-k) \oplus a_{h+1, k-h *} \Lambda(-k) \rightarrow \cdots \rightarrow a_{n-1, n-1 *} \Lambda(-k)$, where the sheaf $a_{n-1, n-1 *} \Lambda(-k)$ is put in degree $2 n-2$. The general term of 
the complex which appears in degree $h_{1}+h_{2}$ is

$$
\bigoplus_{\substack{h_{1} \geq h \\ h_{2} \geq k-h}} a_{h_{1}, h_{2} *} \Lambda(-k)
$$

For each $h_{1}, h_{2}$ the complexes $a_{h_{1}, h_{2} *} \Lambda(-k)\left[-h_{1}-h_{2}\right]$ are $-(2 n-2)$-shifted perverse sheaves, so the complex $R^{k} \psi \Lambda_{X_{1} \times X_{2}}[-k]$ is also $-(2 n-2)$-shifted perverse sheaf.

Proof. Each of the complexes $R^{h} \psi \Lambda_{X_{1}}$ and $R^{k-h} \psi \Lambda_{X_{2}}$ have resolutions in terms of $a_{h_{1 *}}^{1} \Lambda(-h)$ and $a_{h_{2 *}}^{2} \Lambda(-k+h)$ respectively, where $h \leq h_{1} \leq n-1$ and $k-h \leq h_{2} \leq n-1$. We form the double complex associated to the product of these resolutions and the single complex associated to it is a resolution of $R^{h} \psi \Lambda_{X_{1}} \otimes R^{k-h} \psi \Lambda_{X_{2}}[-k]$ of the following form:

$$
\begin{gathered}
a_{h *}^{1} \Lambda(-h) \otimes a_{k-h *}^{2} \Lambda(-k+h) \rightarrow a_{h+1 *}^{1} \Lambda(-h) \otimes a_{k-h *}^{2} \Lambda(-k+h) \oplus a_{h *}^{1} \Lambda(-h) \otimes a_{k-h+1 *}^{2} \Lambda(-k+h) \\
\rightarrow \cdots \rightarrow a_{n-1 *}^{1} \Lambda(-h) \otimes a_{n-1 *}^{2} \Lambda(-k+h)
\end{gathered}
$$

In the above complex, the sheaf $a_{n-1 *}^{1} \Lambda(-h) \otimes a_{n-1 *}^{2} \Lambda(-k+h)$ is put in degree $2 n-2$. Now we use the formula

$$
a_{h_{1}, h_{2} *} \Lambda(-k)=a_{h_{1 *}}^{1} \Lambda(-h) \otimes a_{h_{2} *}^{1} \Lambda(-k+h)
$$

to conclude the first part of the lemma. The complex $a_{h_{1}, h_{2} *} \Lambda(-k)\left[-h_{1}-h_{2}\right]$ is the direct image via $a_{h_{1}, h_{2} *}$ of the complex $\Lambda(-k)\left[-h_{1}-h_{2}\right]$ on $Y^{\left(h_{1}, h_{2}\right)}$. Since $Y^{\left(h_{1}, h_{2}\right)}$ is smooth of dimension $2 n-2-h_{1}-h_{2}$, we know that $\Lambda(-k)\left[-h_{1}-h_{2}\right]$ is a - (2n-2)-shifted perverse sheaf, so its direct image under the finite map $a_{h_{1}, h_{2} *}$ is also a $-(2 n-2)$-shifted perverse sheaf. We've just seen that each $R^{h} \psi \Lambda_{X_{1}} \otimes$ $R^{k-h} \psi \Lambda_{X_{2}}$ can be obtained from successive extensions of factors of the form 
$a_{h_{1}, h_{2} *} \Lambda(-k)\left[-h_{1}-h_{2}\right]$ and since the category of $-(2 n-2)$-shifted perverse sheaves is stable under extensions we deduce that $R^{h} \psi \Lambda_{X_{1}} \otimes R^{k-h} \psi \Lambda_{X_{2}}[-k]$ is a $-(2 n-2)$-shifted perverse sheaf. Now $R^{k} \psi \Lambda_{X_{1} \times X_{2}}[-k]=\bigoplus_{h=0}^{k} R^{h} \psi \Lambda_{X_{1}} \otimes$ $R^{k-h} \psi \Lambda_{X_{2}}[-k]$, so it is also a $-(2 n-2)$-shifted perverse sheaf.

Corollary 4.2.2. $R \psi \Lambda_{X_{1} \times X_{2}}$ is a-(2n-2)-shifted perverse sheaf. The standard truncation $\tau_{\leq k} R \psi \Lambda_{X_{1} \times X_{2}}$ is a filtration by $-(2 n-2)$-shifted perverse sheaves and the graded pieces of this filtration are the $R^{k} \psi \Lambda_{X_{1} \times X_{2}}[-k]$.

Proof. The proof is exactly the same as that of Corollary 4.1.4. It suffices to show that each $\tau_{\leq k} R \psi \Lambda$ is a $-(2 n-2)$-shifted perverse sheaf and we can do this by induction, using the distinguished triangle

$$
\left(\tau_{\leq k-1} R \psi \Lambda_{X_{1} \times X_{2}}, \tau_{\leq k} R \psi \Lambda_{X_{1} \times X_{2}}, R^{k} \psi \Lambda_{X_{1} \times X_{2}}[-k]\right)
$$

Once everything is proven to be in an abelian category, the distinguished triangle becomes a short exact sequence and we get a filtration on $R \psi \Lambda_{X_{1} \times X_{2}}$ with its desired graded pieces.

Now we can deduce that there is a map

$$
\bar{\nu}: R^{k} \psi \Lambda_{X_{1} \times X_{2}}[-k] \rightarrow R^{k-1} \psi \Lambda_{X_{1} \times X_{2}}[-(k-1)] .
$$

Indeed, since $T$ acts trivially on the cohomology sheaves of $R \psi \Lambda_{X_{1} \times X_{2}}$, we deduce that $\nu$ sends $\tau_{\leq k} R \psi \Lambda_{X_{1} \times X_{2}}$ to $\tau_{\leq k-1} R \psi \Lambda_{X_{1} \times X_{2}}$, which induces $\bar{\nu}$. It remains to check that this induced map $\bar{\nu}$ restricted to $R^{h} \psi \Lambda_{X_{1}} \otimes R^{k-h} \psi \Lambda_{X_{2}}$ is the same map as $\bar{\nu}_{1} \otimes 1+1 \otimes \bar{\nu}_{2}$, sending

$R^{h} \psi \Lambda_{X_{1}} \otimes R^{k-h} \psi \Lambda_{X_{2}}[-k] \rightarrow\left(R^{h-1} \psi \Lambda_{X_{1}} \otimes R^{k-h} \psi \Lambda_{X_{1}} \oplus R^{h} \psi \Lambda_{X_{1}} \otimes R^{k-h-1} \psi \Lambda_{X_{2}}\right)[-(k-1)]$.

First notice that for each $0 \leq h \leq k \leq n-1$ the complex $\tau_{\leq h} R \psi \Lambda_{X_{1}} \otimes$ 
$\tau_{\leq k-h} R \psi \Lambda_{X_{2}}$ is a $-(2 n-2)$-shifted perverse sheaf, because it is the external tensor product of $-(n-1)$-shifted perverse sheaves on $X_{1}$ and on $X_{2}$. (See proposition 4.2.8 of $[\mathrm{BBD}])$. Let

$$
\tau_{\leq h-1} R \psi \Lambda_{X_{1}} \otimes \tau_{\leq k-h} R \psi \Lambda_{X_{2}}+\tau_{\leq h} R \psi \Lambda_{X_{1}} \otimes \tau_{\leq k-h-1} R \psi \Lambda_{X_{2}}
$$

be the image of

$\tau_{\leq h-1} R \psi \Lambda_{X_{1}} \otimes \tau_{\leq k-h} R \psi \Lambda_{X_{2}} \oplus \tau_{\leq h} R \psi \Lambda_{X_{1}} \otimes \tau_{\leq k-h-1} R \psi \Lambda_{X_{2}} \rightarrow \tau_{\leq k-1} R \psi \Lambda_{X_{1} \times X_{2}}$

We have a commutative diagram of $-(2 n-2)$-shifted perverse sheaves

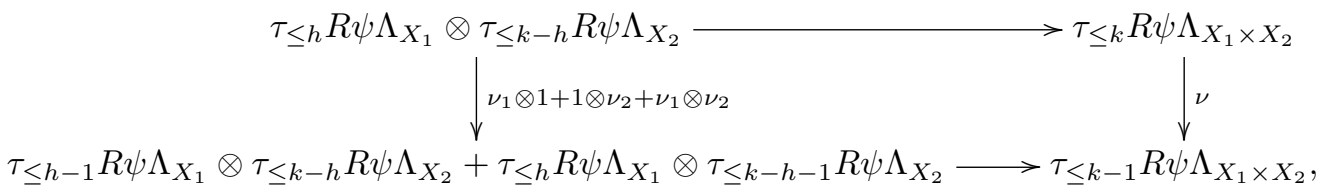

where the horizontal maps are the natural maps of complexes.

Lemma 4.2.3. Assume $\Lambda=\mathbb{Z} / l^{r} \mathbb{Z}$. The image of $R_{h, k-h}=\tau_{\leq h} R \psi \Lambda_{X_{1}} \otimes$ $\tau_{\leq k-h} R \psi \Lambda_{X_{2}}$ in $R^{k} \psi \Lambda[-k]$ is $R^{h} \psi \Lambda_{X_{1}} \otimes R^{k-h} \psi \Lambda_{X_{2}}[-k]$.

Proof. The map of perverse sheaves $R_{h, k-h} \rightarrow \tau_{\leq k} R \psi \Lambda_{X_{1} \times X_{2}} \rightarrow R^{k} \psi \Lambda[-k]$ factors through

$$
R^{h} \psi \Lambda_{X_{1}} \otimes R^{k-h} \psi \Lambda_{X_{2}}[-k] \hookrightarrow R^{k} \psi \Lambda[-k]
$$

This can be checked on the level of complexes. We only need to know that the natural map

$$
R_{h, k-h} \stackrel{g}{\rightarrow} R^{h} \psi \Lambda_{X_{1}} \otimes R^{k-h} \psi \Lambda_{X_{2}}[-k]
$$


is a surjection. This follows once we know that the triangle

$$
R_{h-1, k-l}+R_{h, k-h-1} \stackrel{f}{\rightarrow} R_{h, k-h} \stackrel{g}{\rightarrow} R^{h} \psi \Lambda_{X_{1}} \otimes R^{k-h} \psi \Lambda_{X_{2}}[-k]
$$

is distinguished, since then it has to be a short exact sequence of $-(2 n-2)$ shifted perverse sheaves, so $g$ would be a surjection. To check that the triangle is distinguished, it suffices to compute the fiber of $g$ and check that it is quasiisomorphic to

$$
\mathcal{M}=\tilde{\tau}_{\leq h-1} R \psi \Lambda_{X_{1}} \otimes \tau_{\leq k-h} R \psi \Lambda_{X_{2}}+\tau_{\leq h} R \psi \Lambda_{X_{1}} \otimes \tilde{\tau}_{\leq k-h-1} R \psi \Lambda_{X_{2}}
$$

Let $\mathcal{K}$ be a representative for $R \psi \Lambda_{X_{1}}$ and $\mathcal{L}$ be representative for $R \psi \Lambda_{X_{2}}$. The degree $j<k$ term of $\mathcal{M}$ and of the fiber of $g$ are both equal to

$$
\left(\bigoplus_{i=j-k+l+1}^{h-1} \mathcal{K}^{i} \otimes \mathcal{L}^{j-i}\right) \oplus \mathcal{K}^{j-k+h} \otimes \operatorname{ker}\left(\mathcal{L}^{k-h} \rightarrow \mathcal{L}^{k-h+1}\right) \oplus \operatorname{ker}\left(\mathcal{K}^{h} \rightarrow \mathcal{K}^{h+1}\right) \otimes \mathcal{L}^{j-h}
$$

and the differentials are identical. The last non-zero term $\mathcal{M}^{k}$ in $\mathcal{M}$ appears in degree $k$ and is equal to

$\operatorname{ker}\left(\mathcal{K}^{h} \rightarrow \mathcal{K}^{h+1}\right) \otimes \operatorname{im}\left(\mathcal{L}^{k-h-1} \rightarrow \mathcal{L}^{k-h}\right)+\operatorname{im}\left(\mathcal{K}^{h-1} \rightarrow \mathcal{K}^{h}\right) \otimes \operatorname{ker}\left(\mathcal{L}^{k-h} \rightarrow \mathcal{L}^{k-h+1}\right)$

The main problem is checking that the following map of complexes is a quasiisomorphism

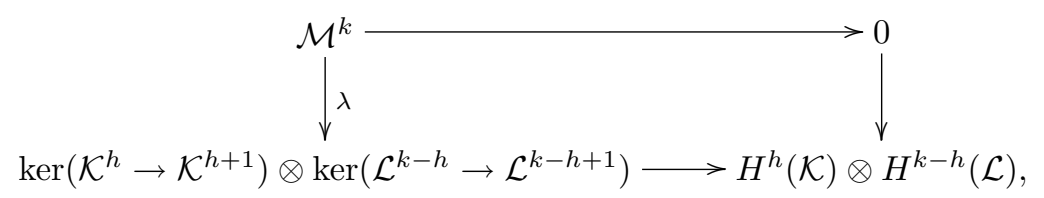

where the left vertical arrow $\lambda$ is the natural inclusion. It is equivalent to prove 
that the object in the lower right corner is the cokernel of $\lambda$. This follows from the Künneth spectral sequence, when computing the cohomology of the product of the two complexes

$$
\begin{gathered}
\tilde{\mathcal{K}}:=\left[\operatorname{im}\left(\mathcal{K}^{h-1} \rightarrow \mathcal{K}^{h}\right) \rightarrow \operatorname{ker}\left(\mathcal{K}^{h} \rightarrow \mathcal{K}^{h+1}\right)\right] \text { and } \\
\tilde{\mathcal{L}}:=\left[\operatorname{im}\left(\mathcal{L}^{k-h-1} \rightarrow \mathcal{L}^{k-h}\right) \rightarrow \operatorname{ker}\left(\mathcal{L}^{k-h} \rightarrow \mathcal{L}^{k-h+1}\right)\right]
\end{gathered}
$$

Indeed, since $H^{1}(\tilde{\mathcal{K}})=R^{h} \psi \Lambda_{X_{1}}$ and $H^{1}(\tilde{\mathcal{L}})=R^{k-h} \psi \Lambda_{X_{2}}$ are both flat over $\Lambda$ the Künneth spectral sequence degenerates. We get $H^{2}(\tilde{\mathcal{K}} \otimes \tilde{\mathcal{L}})=H^{1}(\tilde{\mathcal{K}}) \otimes H^{1}(\tilde{\mathcal{L}})$ and this is exactly the statement that $H^{h}(\mathcal{K}) \otimes H^{k-h}(\mathcal{L})$ is the cokernel of $\lambda$.

Note 4.2.4. The result of this lemma extends to $\Lambda=\mathbb{Q}_{l}$ and $\Lambda=\overline{\mathbb{Q}}_{l}$.

Putting together the above discussion and keeping in mind that the image of $\nu_{1} \otimes \nu_{2}$ in $R^{k-1} \psi \Lambda_{X_{1} \times X_{2}}[-(k-1)]$ is trivial, we conclude the following result.

Proposition 4.2.5. The action of $N$ on $R \psi \Lambda_{X_{1} \times X_{2}}$ induces a map

$$
\bar{\nu}: R^{k} \psi \Lambda_{X_{1} \times X_{2}}[-k] \rightarrow R^{k-1} \psi \Lambda_{X_{1} \times X_{2}}[-(k-1)]
$$

which coincides with $\bar{\nu}_{1} \otimes 1+1 \otimes \bar{\nu}_{2}$ when restricted to $R^{h} \psi \Lambda_{X_{1}} \otimes R^{k-h} \psi \Lambda_{X_{2}}[-k]$ for each $0 \leq h \leq k$.

We now use the decomposition of $R^{k} \psi \Lambda_{X_{1} \times X_{2}}[-k]$ in terms of $R^{h} \psi \Lambda_{X_{1}} \otimes$ $R^{k-h} \psi \Lambda_{X_{2}}[-k]$ for $0 \leq h \leq k$ and the resolution of $R^{h} \psi \Lambda_{X_{1}} \otimes R^{k-h} \psi \Lambda_{X_{2}}[-k]$ in terms of $a_{h_{1}, h_{2} *} \Lambda(-k)\left[-\left(h_{1}+h_{2}\right)\right]$ to get a resolution of $R^{k} \psi \Lambda_{X_{1} \times X_{2}}[-k]$, of the form

$$
\bigoplus_{h_{1}+h_{2}=k} a_{h_{1}, h_{2} *} \Lambda(-k)^{\oplus c_{h_{1} h_{2}}^{k}} \rightarrow \cdots \rightarrow \bigoplus_{h_{1}+h_{2}=k+j} a_{h_{1}, h_{2} *} \Lambda(-k)^{\oplus c_{h_{1}, h_{2}}^{k}} \rightarrow \ldots
$$


where the first term is put in degree $k$ and the coefficients $c_{h_{1}, h_{2}}^{k}$ count how many copies of $a_{h_{1}, h_{2} *} \Lambda(-k)$ show up in the direct sum.

Lemma 4.2.6. Let $c_{h_{1}, h_{2}}^{k}$ be the coefficient of $a_{h_{1}, h_{2} *} \Lambda(-k)\left[-\left(h_{1}+h_{2}\right)\right]$ in the resolution of $R^{k} \psi \Lambda_{X_{1} \times X_{2}}[-k]$. Then

$$
c_{h_{1}, h_{2}}^{k}=\min \left(\min \left(h_{1}, h_{2}\right)+1, h_{1}+h_{2}-k+1, k+1\right) .
$$

Proof. The coefficient $c_{h_{1}, h_{2}}^{k}$ counts for how many values of $0 \leq h \leq k$ the resolution of $R^{h} \psi \Lambda_{X_{1}} \otimes R^{k-h} \psi \Lambda_{X_{2}}$ contains the term $a_{h_{1}, h_{2} *} \Lambda(-k)\left[-\left(h_{1}+h_{2}\right)\right]$. This count is clearly bounded by $k+1$, because there are $k+1$ possible values of $h$. When $h_{1}+h_{2}-k+1 \leq k+1$, the count is

$$
\min \left(\min \left(h_{1}, h_{2}\right)+1, h_{1}+h_{2}-k+1\right),
$$

because $a_{h_{1}, h_{2} *} \Lambda(-k)\left[-\left(h_{1}+h_{2}\right)\right]$ will show up in the resolution of $R^{h_{1}-j} \psi \Lambda_{X_{1}} \otimes$ $R^{k-h_{1}+j} \psi \Lambda_{X_{2}}$ for all $0 \leq j \leq h_{1}+h_{2}-k+1$ which satisfy $0 \leq h_{1}-j \leq k$. When both $h_{1}$ and $h_{2}$ are less than $k$, all the $j \in 0, \ldots, h_{1}+h_{2}-k+1$ satisfy the requirement. When $h_{2} \geq k$, there are exactly $h_{1}+1$ values of $j$ which satisfy the requirement and we can treat the case $h_{1} \geq k$ analogously to get $h_{2}+1$ values of $j$. This covers the case $h_{1}+h_{2} \leq 2 k$. In the case $h_{1}+h_{2} \geq 2 k$, we need to count all $0 \leq j \leq k$ which satisfy $0 \leq h_{1}-j \leq k$. The result is

$$
\min \left(\min \left(h_{1}, h_{2}\right)+1, k+1\right) .
$$

This completes the determination of $c_{h_{1}, h_{2}}^{k}$.

Note that for all $h_{1}+h_{2} \leq 2 k-2$, we have $c_{h_{1}, h_{2}}^{k} \leq c_{h_{1}, h_{2}}^{k-1}$. For $h_{1}+h_{2}=2 k-1$ 
we always have $\min \left(h_{1}, h_{2}\right)+1 \leq k<k+1$, so that

$$
c_{h_{1}, h_{2}}^{k}=c_{h_{1}, h_{2}}^{k-1}=\min \left(h_{1}, h_{2}\right)+1
$$

However, $c_{k, k}^{k}=k+1>k=c_{k, k}^{k-1}$ and for $h_{1}+h_{2} \geq 2 k$ we have $c_{h_{1}, h_{2}}^{k} \geq c_{h_{1}, h_{2}}^{k-1}$.

We now have an explicit description of

$$
\bar{\nu}: R^{k} \psi \Lambda_{X_{1} \times X_{2}}[-k] \rightarrow R^{k-1} \psi \Lambda_{X_{1} \times X_{2}}[-k]
$$

as a map of complexes with terms of the form $\bigoplus_{h_{1}+h_{2}=k+j} a_{h_{1}, h_{2} *} \Lambda(-k)^{\oplus c_{h_{1}, h_{2}}^{k}}$, which are put in degree $k+j$. Writing $\bar{\nu}=\bar{\nu}_{1} \otimes 1+1 \otimes \bar{\nu}_{2}$ as a map of complexes, we will be able to compute both the kernel and cokernel of $\bar{\nu}$.

We will now restrict to $\Lambda=\mathbb{Q}_{l}$ or $\overline{\mathbb{Q}}_{l}$. In this case, $N=\log T$ is defined, it acts trivially on the cohomology sheaves $R^{k} \psi \Lambda_{X_{1} \times X_{2}}$, so it induces a map

$$
\bar{N}: R^{k} \psi \Lambda_{X_{1} \times X_{2}}[-k] \rightarrow R^{k-1} \psi \Lambda_{X_{1} \times X_{2}}[-k] .
$$

Since $N \equiv T-1\left(\bmod (T-1)^{2}\right)$ and $(T-1)^{2}$ sends $\tau_{\leq k} R \psi \Lambda \rightarrow \tau_{\leq k-2} R \psi \Lambda$ (here $\tau_{\leq k}$ denote the truncation functors for $\mathbb{Q}_{l}$ or $\overline{\mathbb{Q}}_{l}$-sheaves) the two maps $\bar{N}$ and $\bar{\nu}$ coincide and we will work with $\bar{N}$ from now on, to which the results of Proposition 4.2.5 apply.

First we need a preliminary result, which will allow us to compute the kernels and cokernels of certain morphisms of $-(2 n-2)$-shifted perverse sheaves. Note that, while $D_{c}^{b}(Y, \Lambda)$ is not known to be a derived category of some category of $\Lambda$-sheaves, being constructed as a "projective limit" of derived categories, it is nevertheless endowed with a standard $t$-structure whose core is the category of $\Lambda$-sheaves. Therefore, by Proposition 3.1.10 of [BBD], we have a realization functor from the bounded derived category of $\Lambda$-sheaves to $D_{c}^{b}(Y, \Lambda)$. Using this 
functor, we think of a bounded complex of $\Lambda$-sheaves as an element in $D_{c}^{b}(Y, \Lambda)$ and of a morphism of complexes as a morphism in $D_{c}^{b}(Y, \Lambda)$.

Lemma 4.2.7. Assume that $f: \mathcal{C} \rightarrow \mathcal{D}$ is a morphism in $D_{c}^{b}(Y, \Lambda)$ which satisfies the following: $\mathcal{C}$ and $\mathcal{D}$ are (the image of) complexes $\left(\mathcal{C}^{k}\right)_{k \in \mathbb{Z}}$ and $\left(\mathcal{D}^{k}\right)_{k \in \mathbb{Z}}$ of l-adic sheaves and $f$ is a map of complexes defined degree by degree as $f^{k}: \mathcal{C}^{k} \rightarrow \mathcal{D}^{k}$. Assume that each $f^{k}$ is injective as a map of sheaves. Let $\overline{\mathcal{D}}^{k}=\operatorname{coker}\left(f^{k}\right)$ and let $\overline{\mathcal{D}}$ be (the image in $D_{c}^{b}(Y, \Lambda)$ of) the complex with terms $\overline{\mathcal{D}}^{k}$ and differential $\bar{d}$ induced by the differential d of $\mathcal{D}$. Assume that the short exact sequence of sheaves

$$
0 \rightarrow \mathcal{C}^{k} \stackrel{f^{k}}{\rightarrow} \mathcal{D}^{k} \rightarrow \overline{\mathcal{D}}^{k} \rightarrow 0
$$

is splittable. Assume also that $\mathcal{C}^{k}[-k]$ and $\mathcal{D}^{k}[-k]$ are $-(2 n-2)$-shifted perverse sheaves.

Then $\overline{\mathcal{D}}^{k}[-k]$ is a $-(2 n-2)$-shifted perverse sheaf and thus so is $\overline{\mathcal{D}}$ (since it is an extension of $\overline{\mathcal{D}}^{k}[-k]$ for finitely many $\left.k\right)$. Moreover, the following is an exact sequence of $-(2 n-2)$-shifted perverse sheaves

$$
0 \rightarrow \mathcal{C} \rightarrow \mathcal{D} \rightarrow \overline{\mathcal{D}} \rightarrow 0
$$

Proof. $\overline{\mathcal{D}}^{k}[-k]$ is a $-(2 n-2)$-shifted perverse sheaf because it is a direct factor of $\mathcal{D}^{k}[-k]$ and so $\overline{\mathcal{D}}$ is also a $-(2 n-2)$-shifted perverse sheaf. If $\Lambda$ was torsion, then we could identify the category $D_{c}^{b}(Y, \Lambda)$ with a full subcategory of the derived category of the category of sheaves of $\Lambda$-modules (whose objects have bounded constructible cohomology) and the corollary would follow from a standard diagram chase in the derived category of an abelian category. However, the cases we are interested in are $\Lambda=\mathbb{Q}_{l}$ or $\overline{\mathbb{Q}}_{l}$. It is possible that by checking the definition of the category $D_{c}^{b}(Y, \Lambda)$ carefully, we could ensure that a version 
of the diagram chase applies to our case. However, an alternative approach uses Beilinson's result which identifies $D_{c}^{b}(Y, \Lambda)$ with the derived category of perverse sheaves on $Y$, see [Be].

We see that the map $f: \mathcal{C} \rightarrow \mathcal{D}$ is injective, since we can think of it as a map of filtered objects, which is injective on the $k$ th graded pieces for each $k$. Indeed $\mathcal{C}$ is a successive extension of the $-(2 n-2)$-shifted perverse sheaves $\mathcal{C}^{k}[-k]$ and $\mathcal{D}$ is a successive extension of $\mathcal{D}^{k}[-k]$ and the fact that $f$ is a map of complexes implies that $f$ respects these extensions. Let $k$ be the largest integer for which either of $\mathcal{C}^{k}$ and $\mathcal{D}^{k}$ is non-zero. We have the commutative diagram of exact sequences

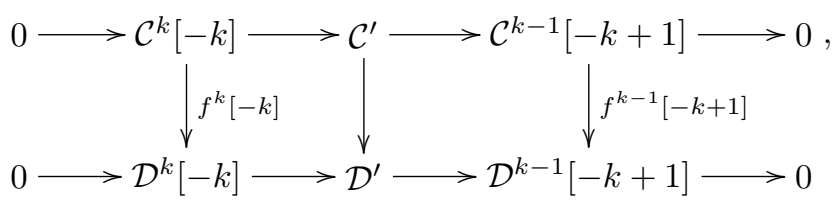

where the arrows on the left and on the right are injective. The fact that the middle map is also injective follows from a standard diagram chase. (Note that we are working in the category of $-(2 n-2)$-shifted perverse sheaves, which is abelian, so we can perform diagram chases by [Re].) The injectivity of $f$ follows by induction.

By a repeated application of the snake lemma in the abelian category of $-(2 n-2)$-shifted perverse sheaves, we see that the cokernel of $f$ is a succesive extension of terms of the form $\overline{\mathcal{D}}^{k}[-k]$. In order to identify this cokernel with $\overline{\mathcal{D}}$, it suffices to check that the differential of $\overline{\mathcal{D}}$ coincides in $\operatorname{Ext}^{1}\left(\overline{\mathcal{D}}^{k}[-k], \overline{\mathcal{D}}^{k-1}[-k+\right.$ 1]) with the extension class which defines the cokernel. To check this, it is enough 
to see that the following square is commutative

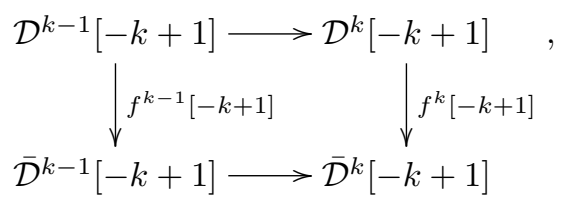

where the top (resp. bottom) horizontal map is the boundary map obtained from considering the distinguished triangle $\left(\mathcal{D}^{k}[-k], \mathcal{D}^{\prime}, \mathcal{D}^{k-1}[-k+1]\right)$ (resp. $\left.\left(\overline{\mathcal{D}}^{k}[-k], \overline{\mathcal{D}}^{\prime}, \overline{\mathcal{D}}^{k-1}[-k+1]\right)\right)$ in $D_{c}^{b}(Y, \Lambda)$. The top boundary map is the differential of $\mathcal{D}$ and if the square is commutative, then the bottom map must be the differential of $\overline{\mathcal{D}}$. The commutativity can be checked by hand, by making the boundary maps explicit using the construction of the cone. (There is a natural map

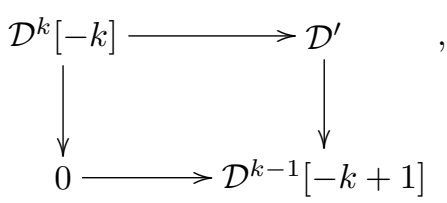

which is a quasi-isomorphism in $D_{c}^{b}(Y, \Lambda)$. The boundary map of the distinguished triangle is obtained by composing the inverse of this quasi-isomorphism with the natural map

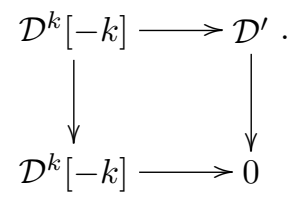

The same construction works for $\overline{\mathcal{D}}$ and it is straightforward to check the commutativity now.)

Lemma 4.2.8. Let $k \geq 1$. Consider the map

$$
\bar{N}: R^{k} \psi \Lambda_{X_{1} \times X_{2}}[-k] \rightarrow R^{k-1} \psi \Lambda_{X_{1} \times X_{2}}[-(k-1)] .
$$


Define the complex

$\mathcal{P}_{k}=\left[a_{k, k *} \Lambda(-k) \stackrel{\wedge \delta}{\rightarrow} a_{k, k+1 *} \Lambda(-k) \oplus a_{k+1, k *} \Lambda(-k) \rightarrow \cdots \stackrel{\wedge \delta}{\rightarrow} a_{n-1, n-1 *} \Lambda(-k)\right]$,

where $a_{k, k *} \Lambda(-k)$ is put in degree $2 k$. The factor $a_{h_{1}, h_{2} *} \Lambda(-k)$ appears in the resolution of $\mathcal{P}$ in degree $h_{1}+h_{2}$ whenever $h_{1}, h_{2} \in\{k, k+1, \ldots, n-1\}$. Also define the complex

$$
\mathcal{R}_{k}=\left[\oplus_{j=0}^{k-1} a_{j, k-1-j *} \Lambda(-(k-1)) \rightarrow \cdots \rightarrow a_{k-1, k-1 *} \Lambda((-(k-1))],\right.
$$

where the first term is put in degree $k-1$ and the term $a_{h_{1}, h_{2} *} \Lambda(-(k-1))$ appears in degree $h_{1}+h_{2}$ whenever $h_{1}, h_{2} \in\{0,1, \ldots, k-1\}$.

Then $\mathcal{P}_{k} \simeq \operatorname{ker}(\bar{N})$ and $\mathcal{R}_{k} \simeq \operatorname{coker}(\bar{N})$.

Proof. Note that both $\mathcal{P}_{k}$ and $\mathcal{R}_{k}$ are $-(2 n-2)$-shifted perverse sheaves, by the same argument we've used before. The proof will go as follows: we will first define a map $\mathcal{P}_{k} \rightarrow R^{k} \psi \Lambda_{X_{1} \times X_{2}}[-k]$ and check that $\bar{N}$ kills the image of $\mathcal{P}_{k}$. We use Lemma 4.2.7 to check that the map $\mathcal{P}_{k} \rightarrow R^{k} \psi \Lambda_{X_{1} \times X_{2}}[-k]$ is an injection and to compute its cokernel $\mathcal{Q}_{k}$. Then we check using Lemma 4.2.7 again that the induced map $\mathcal{Q}_{k} \rightarrow R^{k-1} \psi \Lambda[-(k-1)]$ is an injection and we identify its cokernel with $\mathcal{R}_{k}$.

For the first step, note that it suffices to define the maps

$$
f^{h_{1}, h_{2}}: a_{h_{1}, h_{2} *} \Lambda(-k) \rightarrow a_{h_{1}, h_{2} *} \Lambda(-k)^{\oplus(k+1)}
$$

for all $h_{1}, h_{2} \geq k$ and we do so by $x \mapsto\left(x,-x, \ldots,(-1)^{k} x\right)$. These maps are clearly compatible with the differentials $\wedge \delta$, so they induce a map $f: \mathcal{P}_{k} \rightarrow$ $R^{k} \psi \Lambda_{X_{1} \times X_{2}}[-k]$ (this is a map of complexes between $\mathcal{P}$ and the standard rep- 
resentative of $\left.R^{k} \psi \Lambda_{X_{1} \times X_{2}}[-k]\right)$. Moreover, we can check that the restriction

$$
\bar{N}: a_{h_{1}, h_{2} *} \Lambda(-k)^{\oplus(k+1)} \rightarrow a_{h_{1}, h_{2} *} \Lambda(-k)^{\oplus k}
$$

sends $\left(x,-x, \ldots,(-1)^{k} x\right) \mapsto(0, \ldots, 0)$.

Indeed, the $j$ th factor $a_{h_{1}, h_{2} *} \Lambda(-k)$ appears in the resolution of $R^{j} \psi \Lambda_{X_{1}} \otimes$ $R^{k-j} \psi \Lambda_{X_{2}}[-k]$. The latter object is sent by $\bar{N}_{1} \otimes 1$ to $R^{j-1} \psi \Lambda_{X_{1}} \otimes R^{k-j} \psi \Lambda_{X_{2}}[-(k-$ 1)] for $1 \leq j \leq k$ and by $1 \otimes \bar{N}_{2}$ to $R^{j} \psi \Lambda_{X_{1}} \otimes R^{k-1-j} \psi \Lambda_{X_{2}}[-(k-1)]$ for $0 \leq j \leq k-1$. We also know that $\bar{N}_{1} \otimes 1$ kills $R^{0} \psi \Lambda_{X_{1}} \otimes R^{k} \psi \Lambda_{X_{2}}[-k]$ and similarly $1 \otimes \bar{N}_{2}$ kills $R^{k} \psi \Lambda_{X_{1}} \otimes R^{0} \psi \Lambda_{X_{2}}[-k]$. By Lemma 4.1.6, we find that for $1 \leq j \leq k-1$

$$
(0, \ldots, 0, x, 0 \ldots, 0) \mapsto\left(0, \ldots, x \otimes t_{l}(T), x \otimes t_{l}(T), 0 \ldots, 0\right),
$$

where the term $x$ is put in position $j$ and the terms $x \otimes t_{l}(T)$ are put in positions $j-1$ and $j$. We also have

$$
(x, 0, \ldots, 0) \mapsto\left(x \otimes t_{l}(T), 0, \ldots, 0\right) \text { and }(0, \ldots, 0, x) \mapsto\left(0, \ldots, 0, x \otimes t_{l}(T)\right)
$$

Thus, we find that $\bar{N}$ sends

$\left(x,-x, \ldots,(-1)^{k} x\right) \mapsto\left(x \otimes t_{l}(T)-x \otimes t_{l}(T), \ldots,(-1)^{k-1} x \otimes t_{l}(T)+(-1)^{k} x \otimes t_{l}(T)\right)$, and the term on the right is $(0, \ldots, 0)$. Since we have exhibited $\bar{N} \circ f$ as a chain map and we've checked that it vanishes degree by degree, we conclude that $\bar{N} \circ f=0$. Thus, $f\left(\mathcal{P}_{k}\right) \subseteq \operatorname{ker} \bar{N}$.

Note that for all $h_{1}, h_{2} \geq k$ we can identify the quotient of $a_{h_{1}, h_{2} *} \Lambda(-k)^{\oplus(k+1)}$ 
by $f^{h_{1}, h_{2}}\left(a_{h_{1}, h_{2} *} \Lambda(-k)\right)$ with $a_{h_{1}, h_{2} *} \Lambda(-k)^{\oplus k}$. The resulting exact sequence

$$
0 \rightarrow a_{h_{1}, h_{2} *} \Lambda(-k) \stackrel{f^{h_{1}, h_{2}}}{\rightarrow} a_{h_{1}, h_{2} *} \Lambda(-k)^{\oplus(k+1)} \rightarrow a_{h_{1}, h_{2} *} \Lambda(-k)^{\oplus k} \rightarrow 0
$$

is splittable, because the third term is free over $\Lambda$. By Lemma 4.2.7, the map $f: \mathcal{P}_{k} \rightarrow R^{k} \psi \Lambda_{X_{1} \times X_{2}}[-k]$ is injective and we can identify degree by degree the complex $\mathcal{Q}_{k}$ representing the cokernel of $f$. In degrees less than $2 k-1$, the terms of $\mathcal{Q}_{k}$ are the same as those of $R^{k} \psi \Lambda_{X_{1} \times X_{2}}[-k]$ and in degrees at least $2 k-1$, they are the terms of $R^{k-1} \psi \Lambda_{X_{1} \times X_{2}}[-k+1]$.

To prove that the induced map $\mathcal{Q}_{k} \rightarrow R^{k-1} \psi \Lambda_{X_{1} \times X_{2}}[-(k-1)]$ is injective it suffices to check degree by degree and the proof is analogous to the one for $f: \mathcal{P}_{k} \rightarrow R^{k} \psi \Lambda_{X_{1} \times X_{2}}[-k]$. The cokernel is identified with $\mathcal{R}_{k}$ degree by degree, via the exact sequence

$0 \rightarrow a_{h_{1}, h_{2} *} \Lambda(-k)^{\oplus(k-1)} \stackrel{\bar{N}^{h_{1}, h_{2}}}{\rightarrow} a_{h_{1}, h_{2} *} \Lambda(-(k-1))^{\oplus k} \rightarrow a_{h_{1}, h_{2} *} \Lambda(-(k-1)) \rightarrow 0$

for $0 \leq h_{1}, h_{2} \leq k-1$.

Note. 1. The complex $\mathcal{P}_{k}$ has as its factors exactly the terms $a_{h_{1}, h_{2} *} \Lambda(-k)\left[-\left(h_{1}+\right.\right.$ $\left.\left.h_{2}\right)\right]$ for which $c_{h_{1}, h_{2}}^{k}-c_{h_{1}, h_{2}}^{k-1}=1$, while $\mathcal{R}_{k}$ has as its factors the terms $a_{h_{1}, h_{2} *} \Lambda(-(k-$ 1)) $\left[-\left(h_{1}+h_{2}\right)\right]$ for which $c_{h_{1}, h_{2}}^{k-1}-c_{h_{1}, h_{2}}^{k}=1$.

2. Another way to express the kernel of $\bar{N}$ is as the image of $R^{2 k} \psi \Lambda_{X_{1} \times X_{2}}[-2 k]$ in $R^{k} \psi \Lambda_{X_{1} \times X_{2}}[-k]$ under the map

$$
\bar{N}_{1}^{k} \otimes 1-\bar{N}_{1}^{k-1} \otimes \bar{N}_{2}+\cdots+(-1)^{k} 1 \otimes \bar{N}_{2}^{k} .
$$

This follows from Lemmas 4.1.6 and 4.2.8.

Corollary 4.2.9. The filtration of $R \psi \Lambda_{X_{1} \times X_{2}}$ by $\tau_{\leq k} R \psi \Lambda_{X_{1} \times X_{2}}$ induces a filtration on $\operatorname{ker} N$. The first graded piece of this filtration $G r^{1} \operatorname{ker} N$ is $R^{0} \psi \Lambda_{X_{1} \times X_{2}}$. 
The graded piece $G r^{k+1}$ ker $N$ of this filtration is $\mathcal{P}_{k}$.

Proof. We've already seen that $N$ maps all of $R^{0} \psi \Lambda_{X_{1} \times X_{2}}$ to 0 , since $T$ acts trivially on the cohomology of $R \psi \Lambda_{X_{1} \times X_{2}}$. This identifies the first graded piece to be $R^{0} \psi \Lambda_{X_{1} \times X_{2}}$.

In order to identify the $(k+1)$ st graded piece, we will once more pretend that our shifted perverse sheaves have elements. We can do this since the $(2-2 n)$ shifted perverse sheaves form an abelian category and we only need to do this in order to simplify the exposition. First notice that $g^{k}{ }^{k} \operatorname{ker} N \subseteq \mathcal{P}_{k}$, since anything in the kernel of $N$ reduces to something in the kernel of $\bar{N}$.

So it suffices to show that any $x \in \mathcal{P}_{k}$ lifts to some $\tilde{x} \in \operatorname{ker} N$. Pick any $\tilde{x} \in \tau_{\leq k} R \psi \Lambda_{X_{1} \times X_{2}}$ lifting $x$. Since $\bar{N}$ sends $x$ to 0 , we conclude that $N$ maps $\tilde{x}$ to $\tau_{\leq k-2} R \psi \Lambda_{X_{1} \times X_{2}}$. The image of $N \tilde{x}$ in $R^{k-2} \psi \Lambda_{X_{1} \times X_{2}}[-k+2]$ depends on our choice of the lift $\tilde{x}$. However, the image of $N \tilde{x}$ in $\mathcal{R}_{k-1}$ only depends on $x$. If we can show that that image is 0 , we conclude that we can pick a lift $\tilde{x}$ such that $N \tilde{x} \in \tau_{\leq k-3} R \psi \Lambda$. We can continue applying the same argument while modifying our choice of lift $\tilde{x}$, such that $N \tilde{x} \in \tau_{\leq k-j} R \psi \Lambda_{X_{1} \times X_{2}}$ for larger and larger $j$. In the end we see that $N \tilde{x}=0$.

It remains to check that the map $\mathcal{P}_{k} \rightarrow \mathcal{R}_{k-1}$ sending $x \in \mathcal{P}_{k}$ to the image of $N \tilde{x}$ in $\mathcal{R}_{k-1}$ is 0 . We can see this by checking that any map $\mathcal{P}_{k} \rightarrow \mathcal{R}_{k-1}$ is 0 . Indeed, we have the following decompositions of $\mathcal{P}_{k}$ and $\mathcal{R}_{k-1}$ as $(2-2 n)$-shifted perverse sheaves:

$\mathcal{P}_{k}=\left[a_{k, k *} \Lambda(-k) \stackrel{\wedge \delta}{\rightarrow} a_{k, k+1 *} \Lambda(-k) \oplus a_{k+1, k *} \Lambda(-k) \rightarrow \cdots \stackrel{\wedge \delta}{\rightarrow} a_{n-1, n-1 *} \Lambda(-k)\right]$ and $\mathcal{R}_{k-1}=\left[\oplus_{j=0}^{k-1} a_{j, k-2-j *} \Lambda(-(k-2)) \rightarrow \cdots \rightarrow a_{k-2, k-2 *} \Lambda((-(k-2))]\right.$.

Each of the factors $a_{h_{1}, h_{2} *} \Lambda$ is a direct sum of factors of the form $a_{J_{1}, J_{2} *} \Lambda$, where $\operatorname{card} J_{i}=h_{i}$ for $i=1,2$ and $a_{J_{1}, J_{2}}: Y_{J_{1}, J_{2}} \hookrightarrow Y$ is a closed immersion. 
Each factor $a_{J_{1}, J_{2} *} \Lambda$ is a simple $(2-2 n)$-shifted perverse sheaf, so we have decompositions into simple factors for both $\mathcal{P}_{k}$ and $\mathcal{R}_{k-1}$. It is straightforward to see that $\mathcal{P}_{k}$ and $\mathcal{R}_{k-1}$ have no simple factors in common. Thus, any map $\mathcal{P}_{k} \rightarrow \mathcal{R}_{k-1}$ must vanish. The same holds true for any map $\mathcal{P}_{k} \rightarrow \mathcal{R}_{k-j}$ for any $2 \leq j \leq k$.

The filtration with graded pieces $\mathcal{P}_{k}$ on ker $N$ induces a filtration on ker $N / \operatorname{im} N \cap$ ker $N$ whose graded pieces are $\mathcal{P}_{k} / \operatorname{im} \bar{N}$. Indeed, it suffices to check that the image of $\operatorname{im} N$ in $\mathcal{P}_{k}$ coincides with im $\bar{N}$. The simplest way to see this is again by using a diagram chase. First, it is obvious that for

$$
\bar{N}: R^{k} \psi \Lambda_{X_{1} \times X_{2}}[-k] \rightarrow R^{k-1} \psi \Lambda_{X_{1} \times X_{2}}[-k+1]
$$

we have $\operatorname{im} \bar{N} \subseteq G r^{k} \operatorname{im} N$. Now let $x \in G r^{k} \operatorname{im} N$. This means that there exists a lift $\tilde{x} \in \tau_{\leq k-1} R \psi \Lambda_{X_{1} \times X_{2}}$ of $x$ and an element $\tilde{y} \in \tau_{\leq k+j} R \psi \Lambda_{X_{1} \times X_{2}}$ with $0 \leq j \leq 2 n-k$ such that $\tilde{x}=N \tilde{y}$. In order to conclude that $x \in \operatorname{im} \bar{N}$, it suffices to show that we can take $j=0$. In the case $j \geq 1$, let $y \in R^{k+j} \psi \Lambda_{X_{1} \times X_{2}}$ be the image of $\tilde{y}$. We have $\bar{N} y=0$ and in this case we've seen in the proof of Corollary 4.2.9 that we can find $\tilde{y}^{(1)} \in \tau_{\leq k+j-1} R \psi \Lambda_{X_{1} \times X_{2}}$ such that $N\left(\tilde{y}-\tilde{y}^{(1)}\right)=0$. In other words, $\tilde{x}=N \tilde{y}^{(1)}$ and we can replace $j$ by $j-1$. After finitely many steps, we can find $\tilde{y}^{(j)} \in \tau_{\leq k} R \psi \Lambda_{X_{1} \times X_{2}}$ such that $\tilde{x}=N \tilde{y}^{(j)}$. Thus, $x \in \operatorname{im} \bar{N}$.

Lemma 4.2.10. The filtration of $R \psi \Lambda_{X_{1} \times X_{2}}$ by $\tau_{\leq k} R \psi \Lambda$ induces a filtration on $\operatorname{ker} N / \operatorname{im} N \cap \operatorname{ker} N$ with the $(k+1)$-st graded piece $a_{k, k *} \Lambda(-k)[-2 k]$ for $0 \leq k \leq n-1$.

Proof. First, we need to compute the quotient $R^{0} \psi \Lambda_{X_{1} \times X_{2}} / \mathrm{im} N$, which is the same as $R^{0} \psi \Lambda_{X_{1} \times X_{2}} / \mathcal{Q}_{1}=\mathcal{R}_{1}$ and $\mathcal{R}_{1} \simeq a_{0,0 *} \Lambda$ by Lemma 4.2.8.

Now we must compute for each $k \geq 0$ the quotient of $(2-2 n)$-shifted perverse sheaves $\mathcal{P}_{k} / \mathrm{im} \bar{N}$. This is the same as $\mathcal{P}_{k} / \mathcal{Q}_{k+1}$, which is also the image of $\mathcal{P}_{k}$ 
in $\mathcal{R}_{k+1}$ via

$$
\mathcal{P}_{k} \hookrightarrow R^{k} \psi \Lambda[-k] \rightarrow \mathcal{R}_{k+1}
$$

Recall that we have decompositions for both $\mathcal{P}_{k}$ and $\mathcal{R}_{k+1}$ in terms of simple objects in the category of $(2-2 n)$-shifted perverse sheaves,

$$
\begin{gathered}
\mathcal{P}_{k}=\left[a_{k, k *} \Lambda(-k) \stackrel{\wedge \delta}{\rightarrow} a_{k, k+1 *} \Lambda(-k) \oplus a_{k+1, k *} \Lambda(-k) \rightarrow \cdots \stackrel{\wedge \delta}{\rightarrow} a_{n-1, n-1 *} \Lambda(-k)\right] \\
\text { and } \mathcal{R}_{k+1}=\left[\oplus_{j=0}^{k+1} a_{j, k-j *} \Lambda(-k) \rightarrow \cdots \rightarrow a_{k, k *} \Lambda(-k)\right] .
\end{gathered}
$$

The only simple factors that show up in both decompositions are those that show up in $a_{k, k *} \Lambda(-k)[-2 k]$, so these are the only factors that may have nonzero image in $\mathcal{R}_{k+1}$. Thus, $\mathcal{P}_{k} / \mathrm{im} \bar{N}$ is a quotient of $a_{k, k *} \Lambda(-k)[-2 k]$ and it remains to see that it is the whole thing. As seen in Lemma 4.2.8, the map $\mathcal{P}_{k} \rightarrow \mathcal{R}_{k+1}$ can be described as a composition of chain maps. The composition in degree $2 k$ is the map

$$
a_{k, k *} \Lambda(-k) \hookrightarrow a_{k, k *} \Lambda(-k)^{\oplus k+1} \rightarrow a_{k, k *} \Lambda(-k)
$$

where the inclusion sends $x \mapsto\left(x,-x, \ldots,(-1)^{k+1} x\right)$ and the surjection is a quotient by $(x, x, 0, \ldots, 0),(0, x, x, 0 \ldots, 0), \ldots,(0, \ldots, 0, x, x)$ for $x \in a_{k, k *} \Lambda(-k)$. It is elementary to check that the composition of these two maps is an isomorphism, so we are done.

Analogously, we can compute the kernel and cokernel of

$$
\bar{N}^{j}: R^{k} \psi \Lambda_{X_{1} \times X_{2}}[-k] \rightarrow R^{k-j} \psi \Lambda_{X_{1}}[-k+j]
$$

for $2 \leq j \leq k \leq 2 n-2$ and use this to recover the graded pieces of a filtration on $\operatorname{ker} N^{j} / \operatorname{ker} N^{j-1}$ and on $\left(\operatorname{ker} N^{j} / \operatorname{ker} N^{j-1}\right) /\left(\operatorname{im} N \cap \operatorname{ker} N^{j}\right)$. 
Lemma 4.2.11. Let $2 \leq j \leq 2 n-2$. The filtration of $R \psi \Lambda_{X_{1} \times X_{2}}$ by $\tau_{\leq k} R \psi \Lambda$ induces a filtration on

$$
\left(\operatorname{ker} N^{j} / \operatorname{ker} N^{j-1}\right) /\left(\operatorname{im} N \cap \operatorname{ker} N^{j}\right) .
$$

The first graded piece of this filtration is isomorphic to

$$
\bigoplus_{i=0}^{j-1} a_{i, j-1-i *} \Lambda(-j+1)[-j+1] .
$$

For $k \geq 1$, the $(k+1)$-st graded piece is isomorphic to

$$
\left(\operatorname{ker} \bar{N}^{j} / \operatorname{ker} \bar{N}^{j-1}\right) /\left(\operatorname{im} \bar{N} \cap \operatorname{ker} \bar{N}^{j}\right)
$$

where

$$
\bar{N}^{j}: R^{k+j-1} \psi \Lambda[-(k+j-1)] \rightarrow R^{k-1} \psi \Lambda[-k+1] .
$$

More explicitly, the $(k+1)$-st graded piece is isomorphic to

$$
\bigoplus_{i=1}^{j} a_{k+i-1, k+j-i *} \Lambda(-(k+j-1))[-2 k-j+1] .
$$

Proof. We will prove the lemma by induction on $j$. The base case $j=1$ is proven in Corollary 4.2.9 and Lemma 4.2.10. Assume it is true for $j-1$.

To prove the first claim, note that the first graded piece of

$$
\left(\operatorname{ker} N^{j} / \operatorname{im} N \cap \operatorname{ker} N^{j}\right) /\left(\operatorname{ker} N^{j-1} / \operatorname{im} N \cap \operatorname{ker} N^{j-1}\right)
$$

has to be a quotient of

$$
R^{j-1} \psi \Lambda_{X_{1} \times X_{2}}[-j+1] / \mathcal{Q}_{j} \simeq \mathcal{R}_{j}
$$


This is true because $\tau_{\leq j-1} R \psi \Lambda_{X_{1} \times X_{2}} \subseteq \operatorname{ker} N^{j}$ and $\tau_{\leq j-2} R \psi \Lambda_{X_{1} \times X_{2}} \subseteq \operatorname{ker} N^{j-1}$ and

$$
R^{j-1} \psi \Lambda_{X_{1} \times X_{2}}[-j+1]=\tau_{\leq j-1} R \psi \Lambda_{X_{1} \times X_{2}} / \tau_{\leq j-2} R \psi \Lambda_{X_{1} \times X_{2}} .
$$

More precisely, the first graded piece has to be a quotient of

$$
\mathcal{R}_{j} /\left(\operatorname{ker} N^{j-2} / \operatorname{im} N \cap \operatorname{ker} N^{j-2}\right)
$$

by the second graded piece of

$$
\left(\operatorname{ker} N^{j-1} / \operatorname{ker} N^{j-2}\right) /\left(\operatorname{im} N \cap \operatorname{ker} N^{j-1}\right) .
$$

(Here, we abusively write

$$
\operatorname{ker} N^{j-2} / \operatorname{im} N \cap \operatorname{ker} N^{j-2}
$$

where we mean the image of this object in $R_{j}$.) By the induction hypothesis, this second graded piece is

$$
\bigoplus_{i=1}^{j-1} a_{i, j-i *} \Lambda(-j+1)[-j]
$$

Continuing this argument, we see that in order to get the first graded piece of $\left(\operatorname{ker} N^{j} / \operatorname{ker} N^{j-1}\right) /\left(\operatorname{im} N \cap \operatorname{ker} N^{j}\right)$ we must quotient $\mathcal{R}_{j}$ successively by

$$
\bigoplus_{i=1}^{j-k} a_{k+i-1, j-i *} \Lambda(-j+1)[-k-j+1]
$$

with $k$ going from $j-1$ down to 1 . (This corresponds to quotienting out successively by the $j$ th graded piece of $\operatorname{ker} N /(\operatorname{im} N \cap \operatorname{ker} N)$, the $(j-1)$ st graded piece of $\left(\operatorname{ker} N^{2} / \operatorname{ker} N\right) /\left(\operatorname{im} N \cap \operatorname{ker} N^{2}\right)$ down to the second graded piece of 
$\left(\operatorname{ker} N^{j-1} / \operatorname{ker} N^{j-2}\right) /\left(\operatorname{im} N \cap \operatorname{ker} N^{j-1}\right)$.) We know that

$$
\mathcal{R}_{j}=\left[\oplus_{i=0}^{j-1} a_{i, j-1-i *} \Lambda(-(j-1)) \rightarrow \cdots \rightarrow a_{j-1, j-1 *} \Lambda((-(j-1))]\right.
$$

with general term in degree $k+j-1$ equal to

$$
\oplus_{i=1}^{j-k} a_{k+i-1, j-i *} \Lambda(-(j-1) .
$$

After quotienting out successively, we are left with only the degree $j-1$ term, which is

$$
\oplus_{i=0}^{j-1} a_{i, j+1-i *} \Lambda(-(j-1))[-(j-1)]
$$

as desired.

In order to identify the $(k+1)$-st graded piece of

$$
\left(\operatorname{ker} N^{j} / \operatorname{ker} N^{j-1}\right) /\left(\operatorname{im} N \cap \operatorname{ker} N^{j}\right)
$$

for $k \geq 1$, we first identify the kernel of $\bar{N}^{j}: R^{k+j-1} \psi \Lambda_{X_{1} \times X_{2}} \rightarrow R^{k-1} \psi \Lambda_{X_{1} \times X_{2}}$ as a map of perverse sheaves, as in Lemma 4.2.8. Then we can identify it with the $(k+1)$-st graded piece of ker $N^{j}$ as in Lemma 4.2 .10 and quotient by $\mathcal{Q}_{k+j}$. Finally, we can use induction as above to compute the $(k+1)$-st graded piece of $\left(\operatorname{ker} N^{j} / \operatorname{ker} N^{j-1}\right) /\left(\operatorname{im} N \cap \operatorname{ker} N^{j}\right)$.

Corollary 4.2.12. The above filtration is a direct sum.

Proof. This follows from the decomposition theorem for pure perverse sheaves, Theorem 5.3.8 of [BBD], once we notice that the $(k+1)$ st graded piece of the filtration is a pure $-(2 n-2)$-shifted perverse sheaf of weight $(-2 k-j+1)+$ $2(k+j-1)=j-1$, which is independent of $k$. 
Let

$$
\operatorname{Gr}^{q} \operatorname{Gr}_{p} R \psi \Lambda=\left(\operatorname{ker} N^{p} \cap \operatorname{im} N^{q}\right) /\left(\operatorname{ker} N^{p-1} \cap \operatorname{im} N^{q}\right)+\left(\operatorname{ker} N^{p} \cap \operatorname{im} N^{q+1}\right) .
$$

The monodromy filtration $M_{r} R \psi \Lambda$ has graded pieces $\mathrm{Gr}_{r}^{M} R \psi \Lambda$ isomorphic to

$$
\mathrm{Gr}_{r}^{M} R \psi \Lambda \simeq \bigoplus_{p-q=r} \mathrm{Gr}^{q} \mathrm{Gr}_{p} R \psi \Lambda
$$

by Lemma 2.1 of [Sa2], so to understand the graded pieces of the monodromy filtration it suffices to understand the $\mathrm{Gr}^{q} \mathrm{Gr}_{p} R \psi \Lambda$. Lemma 4.2 .11 exhibits a decomposition of $\mathrm{Gr}^{0} \mathrm{Gr}_{p} R \psi \Lambda$ as a direct sum with the (k+1)-st term isomorphic to

$$
\oplus_{i=1}^{p} a_{k+i-1, k+p-i *} \Lambda(-(k+p-1))[-2 k-p+1] .
$$

The action of $N^{q}$ induces an isomorphism of $\mathrm{Gr}^{0} \mathrm{Gr}_{p+q} R \psi \Lambda$ with $\mathrm{Gr}^{q} \mathrm{Gr}_{p} R \psi \Lambda(q)$, so there is a direct sum decomposition of the latter with the $(k+1)$-st term isomorphic to

$$
\oplus_{i=1}^{p+q} a_{k+i-1, k+p+q-i *} \Lambda(-(k+p-1))[-2 k-p-q+1] .
$$

We can use the spectral sequence associated to a filtration (as in Lemma 5.2.18 of [Sa1]) to compute the terms in the monodromy spectral sequence

$$
\begin{gathered}
E_{1}^{r, m-r}=H^{m}\left(Y_{\overline{\mathbb{F}}}, \mathrm{Gr}_{-r}^{M} R \psi \Lambda\right)=\bigoplus_{p-q=-r} H^{m}\left(Y_{\overline{\mathbb{F}}}, \mathrm{Gr}^{q} \mathrm{Gr}_{p} R \psi \Lambda\right) \\
\Rightarrow H^{m}\left(Y_{\overline{\mathbb{F}}}, R \psi \Lambda\right)=H^{m}\left(X_{\bar{K}}, \Lambda\right) .
\end{gathered}
$$


Corollary 4.2.13. There is a direct sum decomposition

$H^{m}\left(Y_{\overline{\mathbb{F}}}, \operatorname{Gr}^{q} \operatorname{Gr}_{p} R \psi \Lambda\right) \simeq \bigoplus_{k \geq 0} \bigoplus_{i=1}^{p+q} H^{m}\left(Y_{\overline{\mathbb{F}}}, a_{k+i-1, k+p+q-i *} \Lambda(-(k+p-1))[-2 k-p-q+1]\right)$

compatible with the action of $G_{\mathbb{F}}$. This can be rewritten as

$H^{m}\left(Y_{\overline{\mathbb{F}}}, \operatorname{Gr}^{q} \operatorname{Gr}_{p} R \psi \Lambda\right) \simeq \bigoplus_{k \geq 0} \bigoplus_{i=1}^{p+q} H^{m-2 k-p-q+1}\left(Y_{\overline{\mathbb{F}}}^{(k+i-1, k+p+q-i)}, \Lambda(-(k+p-1))\right)$.

\subsection{More general schemes}

In this section, we will explain how the results of the previous section concerning products of strictly semistable schemes apply to more general schemes, in particular to the Shimura varieties $X_{U} / \mathcal{O}_{K}$. In this section, we will use $\Lambda=\mathbb{Q}_{l}$ or $\overline{\mathbb{Q}}_{l}$.

Let $X^{\prime} / \mathcal{O}_{K}$ be a scheme such that the completions of the strict henselizations $\mathcal{O}_{X^{\prime}, s}^{\wedge}$ at closed geometric points $s$ are isomorphic to

$$
W\left[\left[X_{1}, \ldots, X_{n}, Y_{1}, \ldots Y_{n}\right]\right] /\left(X_{1} \cdots X_{r}-\pi, Y_{1} \cdots Y_{s}-\pi\right)
$$

for some indices $i_{1}, \ldots, i_{r}, j_{1}, \ldots, j_{s} \in\{1, \ldots n\}$ and some $1 \leq r, s \leq n$. Also assume that the special fiber $Y^{\prime}$ is a union of closed subschemes $Y_{1, j}^{\prime}$ with $j \in$ $\{1, \ldots n\}$, which are cut out by one local equation, such that if $s$ is a closed geometric point of $Y_{1, j}^{\prime}$, then $j \in\left\{i_{1}, \ldots, i_{r}\right\}$ and $Y_{1, j}^{\prime}$ is cut out in $\mathcal{O}_{X^{\prime}, s}^{\wedge}$ by the equation $X_{j}=0$. Similarly, assume that $Y^{\prime}$ is a union of closed subschemes $Y_{2, j}^{\prime}$ with $j \in\{1, \ldots, n\}$, which are cut out by one local equation such that if $s$ is a closed geometric point of $Y_{2, j}^{\prime}$ then $j \in\left\{j_{1}, \ldots, j_{r}\right\}$ and $Y_{2, j}^{\prime}$ is cut out in $\mathcal{O}_{X^{\prime}, s}^{\wedge}$ by the equation $Y_{j}=0$.

Let $X / X^{\prime}$ be smooth of dimension $m$ and let $Y$ be the special fiber of $X$ and 
$Y_{i, j}=Y_{i, j}^{\prime} \times_{X^{\prime}} X$ for $i=1,2$ and $j=1, \ldots, n$. As in Lemma $2.2 .7, X^{\prime}$ is locally etale over

$$
X_{r, s}=\operatorname{Spec} \mathcal{O}_{K}\left[X_{1}, \ldots, X_{n}, Y_{1}, \ldots Y_{n}\right] /\left(\prod_{i=1}^{r} X_{i}-\pi, \prod_{j-1}^{s} Y_{j}-\pi\right)
$$

so $X$ is locally etale over

$$
X_{r, s, m}=\operatorname{Spec} \mathcal{O}_{K}\left[X_{1}, \ldots, X_{n}, Y_{1}, \ldots Y_{n}, Z_{1}, \ldots, Z_{m}\right] /\left(\prod_{i=1}^{r} X_{i}-\pi, \prod_{j-1}^{s} Y_{j}-\pi\right),
$$

which is a product of strictly semistable schemes. The results of Section 3 apply to $X^{\prime}$ and it is easy to check that they also apply to $X$. In particular, we know that the inertia $I_{K}$ acts trivially on the sheaves of nearby cycles $R^{k} \psi \Lambda$ of $X$ and we have a description of the $R^{k} \psi \Lambda$ in terms of the log structure we put on $X / \operatorname{Spec} \mathcal{O}_{K}$. Let $a_{j}^{i}: Y_{i, j} \rightarrow Y$ denote the closed immersion for $i=1,2$ and $j \in\{1, \ldots n\}$. Then by Corollary 3.2.3, we have an isomorphism

$$
R^{k} \psi \Lambda(k) \simeq \wedge^{k}\left(\left(\oplus_{j=1}^{n} a_{j *}^{1} \Lambda\right) / \Lambda \oplus\left(\oplus_{j=1}^{n} a_{j *}^{2} \Lambda\right) / \Lambda\right)
$$

For $i=1,2$ and $J_{i} \subseteq\{1, \ldots, n\}$, let

$$
Y_{J_{1}, J_{2}}=\left(\bigcap_{j_{1} \in J_{1}} Y_{1, j_{1}}\right) \cap\left(\bigcap_{j_{2} \in J_{2}} Y_{2, j_{2}}\right)
$$

and let $a_{J_{1}, J_{2}}: Y_{J_{1}, J_{2}} \rightarrow Y$ be the closed immersion. Set

$$
Y^{\left(h_{1}, h_{2}\right)}=\bigsqcup_{\# J_{1}=h_{1}+1, \# J_{2}=h_{2}+1} Y_{J_{1}, J_{2}}
$$

and let $a_{h_{1}, h_{2}}: Y^{\left(h_{1}, h_{2}\right)} \rightarrow Y$ be the projection. The scheme $Y^{\left(h_{1}, h_{2}\right)}$ is smooth of dimension $\operatorname{dim} Y-h_{1}-h_{2}$ (we can see this from the strict local rings). 
We can write

$$
R^{k} \psi \Lambda \simeq \bigoplus_{h=0}^{k} \wedge^{h}\left(\left(\oplus_{j=1}^{n} a_{j *}^{1} \Lambda\right) / \Lambda\right) \otimes \wedge^{k-h}\left(\left(\oplus_{j=1}^{n} a_{j *}^{2} \Lambda\right) / \Lambda\right)(-k)
$$

and then define the map of sheaves on $Y$

$$
\theta_{k}: R^{k} \psi \Lambda \rightarrow \sum_{h=0}^{k} a_{h, k-h *} \Lambda(-k)
$$

as a sum of maps for $h$ going from 0 to $k$. First, define for $i=1,2$

$$
\delta_{h_{i}}: \bigwedge^{h_{i}}\left(\left(\oplus_{j=1}^{n} a_{j *}^{i} \Lambda\right) / \Lambda\right) \rightarrow \bigwedge^{h_{i}+1}\left(\oplus_{j=1}^{n} a_{j *}^{i} \Lambda\right)
$$

by sending

$$
a_{j_{1} *}^{i} \Lambda \wedge \cdots \wedge a_{j_{h_{i} *}}^{i} \Lambda \rightarrow \oplus_{j \neq j_{1}, \ldots, j_{h_{i}}} a_{j_{1} *}^{i} \Lambda \wedge \cdots \wedge a_{j_{h_{i} *}}^{i} \Lambda \wedge a_{j *}^{i} \Lambda
$$

via cup product with the canonical map

$$
\Lambda_{Y} \rightarrow \oplus_{j=1}^{n} a_{j *}^{i} \Lambda
$$

More explicitly, on an open $U$ of $Y$ the map sends

$$
\alpha \in \Lambda\left(U \times_{Y} Y_{j_{1}}^{i} \times \cdots \times_{Y} Y_{j_{l_{i}}}^{i}\right)
$$

to

$$
\left(\left.\alpha\right|_{Y_{j}^{i}}, \ldots,\left.\alpha\right|_{Y_{j}^{i}}\right) \in \oplus_{j \neq j_{1}, \ldots, j_{h_{i}}} \Lambda\left(U \times_{Y} Y_{j}^{i} \times Y_{j_{1}}^{i} \cdots \times_{Y} Y_{j_{h_{i}}}^{i}\right)
$$

and it is easy to check that this is well-defined. Then notice that

$$
\wedge^{h+1}\left(\oplus_{j=1}^{n} a_{j *}^{1} \Lambda\right) \otimes \wedge^{k+1-h}\left(\oplus a_{j *}^{2} \Lambda\right) \simeq a_{h, k-h *} \Lambda .
$$


Indeed, for $J_{1}, J_{2} \subseteq\{1, \ldots, n\}$ with $\# J_{1}=h+1, \# J_{2}=k+1-h$ we have

$$
\left(\bigwedge_{j_{1} \in J_{1}} a_{j_{1} *}^{1} \Lambda\right) \otimes\left(\bigwedge_{j_{2} \in J_{2}} a_{j_{2} *}^{2} \Lambda\right) \simeq a_{J_{1}, J_{2} *} \Lambda
$$

because $Y_{J_{1}, J_{2}}=\left(\cap_{j_{1} \in J_{1}} Y_{1, j_{1}}\right) \times_{Y}\left(\cap_{j_{2} \in J_{2}} Y_{2, j_{2}}\right)$ and we can sum the above identity over all $J_{1}, J_{2}$ of the prescribed cardinality.

Lemma 4.3.1. The following sequence is exact

$$
\begin{gathered}
R^{k} \psi \Lambda \stackrel{\theta_{k}}{\rightarrow} \oplus_{h=0}^{k} a_{h, k-h *} \Lambda(-k)^{\oplus c_{h, k-h}^{k}} \rightarrow \oplus_{h=0}^{k+1} a_{h, k+1-h *} \Lambda(-k)^{\oplus c_{h, k+1-h}^{k}} \rightarrow \ldots \\
\rightarrow \oplus_{h=0}^{2 n-2} a_{h, 2 n-2-h *} \Lambda(-k)^{\oplus c_{h, 2 n-2-h}^{k}} \rightarrow 0
\end{gathered}
$$

where the first map is the one defined above and the coefficients $c_{h_{1}, h_{2}}^{k}$ are defined in Lemma 4.2.6. The remaining maps in the sequence are global maps of sheaves corresponding to $\wedge \delta_{1} \pm \wedge \delta_{2}$, where $\delta_{i} \in \oplus_{j=1}^{n} a_{j *}^{i} \Lambda$ is equal to $(1, \ldots, 1)$ for $i=1,2$. These maps are defined on each of the $c_{h_{1}, h_{2}}^{k}$ factors in the unique way which makes them compatible with the maps in the resolution (4.2).

We can think of $\theta_{k}$ as a quasi-isomorphism of $R^{k} \psi \Lambda[-k]$ with the complex

$$
\oplus_{h=0}^{k} a_{h, k-h *} \Lambda(-k)^{\oplus c_{h, k-h}^{k}} \rightarrow \cdots \rightarrow \oplus_{h=0}^{2 n-2} a_{h, 2 n-2-h *} \Lambda(-k)^{\oplus c_{h, 2 n-2-h}^{k}}
$$

where the leftmost term is put in degree $k$.

Proof. It suffices to check exactness locally and we know that $X$ is locally etale over products $X_{1} \times_{\mathcal{O}_{K}} X_{2}$ of strictly semistable schemes. Lemma 4.2.1 proves the above statement in the case of $X_{1} \times_{\mathcal{O}_{K}} X_{2}$ and the corresponding sheaves on $Y$ are obtained by restriction (etale pullback) from the special fiber $Y_{1} \times_{\mathbb{F}} Y_{2}$ of $X_{1} \times \mathcal{O}_{K} X_{2}$.

Corollary 4.3.2. The complex $R \psi \Lambda$ is a- $\operatorname{dim} Y$-shifted perverse sheaf and 
the canonical filtration $\tau_{\leq k} R \psi \Lambda$ with graded pieces $R^{k} \psi \Lambda[-k]$ is a filtration by - $\operatorname{dim} Y$-shifted perverse sheaves. The monodromy operator $N$ sends $\tau_{\leq k} R \psi \Lambda$ to $\tau_{\leq k-1} R \psi \Lambda$ and this induces a map

$$
\bar{N}: R^{k} \psi \Lambda[-k] \rightarrow R^{k-1} \psi \Lambda[-k+1] .
$$

The next step is to understand the action of monodromy $\bar{N}$ and obtain an explicit description of $\bar{N}$ in terms of the resolution of $R^{k} \psi \Lambda$ given by Lemma 4.3.1. This can be done etale locally, since on the nearby cycles for $X_{1} \times \mathcal{O}_{K} X_{2}$ we know that $\bar{N}$ acts as $\bar{N}_{1} \otimes 1+1 \otimes \bar{N}_{2}$ from Proposition 4.2 .5 and we have a good description of $\bar{N}_{1}$ and $\bar{N}_{2}$ from Lemma 4.1.6. However, we present here a different method for computing $\bar{N}$, which works in greater generality.

Proposition 4.3.3. The following diagram is commutative:

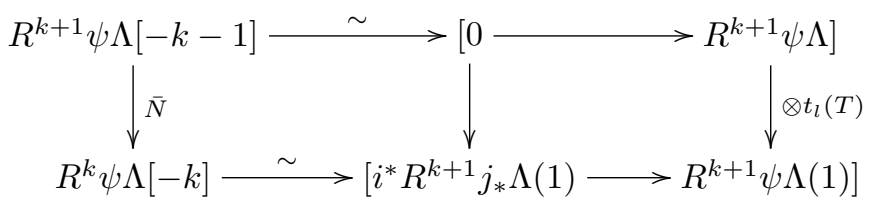

where in the right column the sheaves $R^{k+1} \psi \Lambda$ are put in degree $k+1$.

The proof of this proposition is identical to the proof of part 4 of Lemma 2.5 of [Sa2], which is meant for the strictly semistable case but does not use semistability. The fact that the above formula could hold was suggested to us by reading Ogus' paper [O], which proves an analogous formula for log smooth schemes in the complex analytic world. The same result should hold for any log smooth scheme $X / \mathcal{O}_{K}$ with vertical log structure and where the action of $I_{K}$ on $R^{k} \psi \Lambda$ is trivial for all $k$. 
For $0 \leq k \leq 2 n-2$ define the complex

$\mathcal{L}_{k}:=\left[\oplus_{h=0}^{k}\left(a_{h, k-h *} \Lambda(-k)\right)^{\oplus c_{h, k-h}^{k}} \rightarrow \cdots \rightarrow \oplus_{h=0}^{2 n-2}\left(a_{h, 2 n-2-h *} \Lambda(-k) \oplus^{\oplus c_{h, 2 n-2-h}^{k}}\right]\right.$,

where the sheaves $a_{h, k-h *} \Lambda(-k)$ are put in degree $k$. We will define a map of complexes $f: \mathcal{L}_{k+1} \rightarrow \mathcal{L}_{k}$ degree by degree, as a sum over $h_{1}+h_{2}=k^{\prime}$ of maps

$$
f^{h_{1}, h_{2}} \otimes t_{l}(T): a_{h_{1}, h_{2} *} \Lambda^{\oplus c_{h_{1}, h_{2}}^{k}} \rightarrow a_{h_{1}, h_{2} *} \Lambda(1)^{\oplus c_{h_{1}, h_{2}}^{k}} .
$$

Note that each coefficient $c_{h_{1}, h_{2}}^{k}$ reflects for how many $0 \leq h^{\prime} \leq k$ the term $a_{h_{1}, h_{2} *} \Lambda(-k)$ appears in the resolution of

$$
\bigwedge^{h^{\prime}}\left(\left(\oplus_{j=1}^{n} a_{j *}^{1} \Lambda\right) / \Lambda\right) \otimes \bigwedge^{k-h^{\prime}}\left(\left(\oplus_{j=1}^{n} a_{j *}^{2} \Lambda\right) / \Lambda\right)(-k)
$$

The set of such $h^{\prime}$ has cardinallity $c_{h_{1}, h_{2}}^{k}$ and is always a subset of consecutive integers in $\{1, \ldots, k\}$. Denote the set of $h^{\prime}$ by $C_{h_{1}, h_{2}}^{k}$. Thus, we can order the terms $a_{h_{1}, h_{2} *} \Lambda$ by $h^{\prime}$ and get a basis for $\left(a_{h_{1}, h_{2} *} \Lambda\right)^{\oplus c_{h_{1}, h_{2}}^{k}}$ over $a_{h_{1}, h_{2} *} \Lambda$. It is easy to explain what $f^{h_{1}, h_{2}}$ does to each element of $C_{h_{1}, h_{2}}^{k+1}$ : it sends

$$
h^{\prime} \in C_{h_{1}, h_{2}}^{k+1} \mapsto\left\{h^{\prime}-1, h^{\prime}\right\} \cap C_{h_{1}, h_{2}}^{k}
$$

When both $h^{\prime}-1, h^{\prime} \in C_{h_{1}, h_{2}}^{k}$, the element of the basis of $\left(a_{h_{1}, h_{2} *} \Lambda\right)^{\oplus c_{h_{1}, h_{2}}^{k+1}}$ given by $(0, \ldots 0,1,0, \ldots, 0)$ where the 1 appears in the position corresponding to $h^{\prime}$ is sent to the element of the basis of $\left(a_{h_{1}, h_{2} *} \Lambda\right)^{\oplus c_{h_{1}, h_{2}}^{k}}$ given by $(0, \ldots, 0,1,1,0, \ldots, 0)$ where the two 1 's are in positions corresponding to $h^{\prime}-1$ and $h^{\prime}$. If $h^{\prime}-1 \notin C_{h_{1}, h_{2}}^{k}$ but $h^{\prime} \in C_{h_{1}, h_{2}}^{k}$ then $h^{\prime}=0$ and $(1,0, \ldots, 0) \mapsto$ $(1,0 \ldots, 0)$. If $h^{\prime}-1 \in C_{h_{1}, h_{2}}^{k}$ but $h^{\prime} \notin C_{h_{1}, h_{2}}^{k}$ then $h^{\prime}=k+1$ and $(0, \ldots, 0,1) \mapsto$ $(0, \ldots, 0,1)$. This completes the definition of $f^{h_{1}, h_{2}}$. 
Corollary 4.3.4. The following diagram is commutative

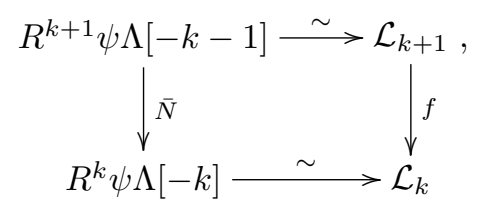

The map $f$ is a map of complexes, which acts degree by degree as

$$
\sum_{h_{1}+h_{2}=k^{\prime}} f^{h_{1}, h_{2}}[-k-1] \otimes t_{l}(T),
$$

where $f^{h_{1}, h_{2}}: a_{h_{1}, h_{2} *} \Lambda^{\oplus c_{h_{1}, h_{2}}^{k}} \rightarrow a_{h_{1}, h_{2} *} \Lambda^{\oplus c_{h_{1}, h_{2}}^{k}}$ was defined above.

Proof. This can be checked etale locally, using Proposition 4.2.5, which states that $\bar{N}=\bar{N}_{1} \otimes 1+1 \otimes \bar{N}_{2}$ over a product $X_{1} \times_{\mathcal{O}_{K}} X_{2}$ of strictly semistable schemes and using the fact that each of the $\bar{N}_{i}$ can be described as

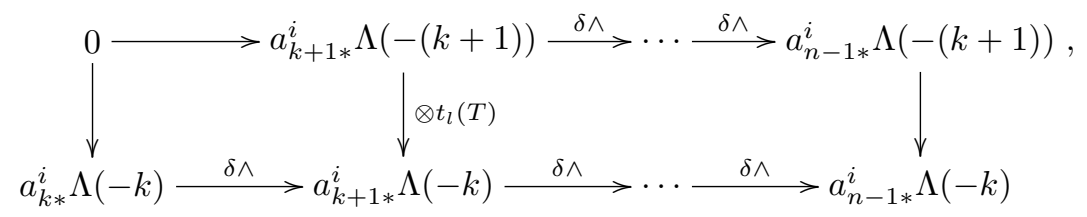

for $i=1,2$.

This can also be checked globally, by using Proposition 4.3.3 to replace the leftmost column of our diagram by

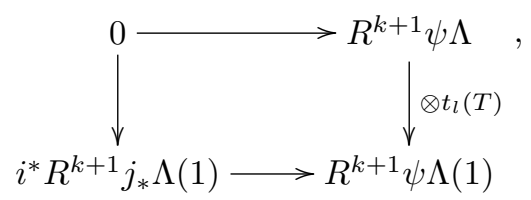

where the left column is put in degree $k$. In fact, it suffices to understand the 
map of complexes

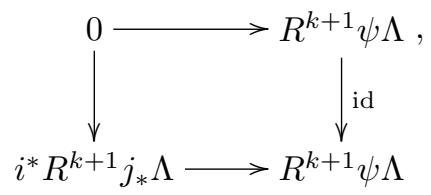

and check that it is compatible with the map

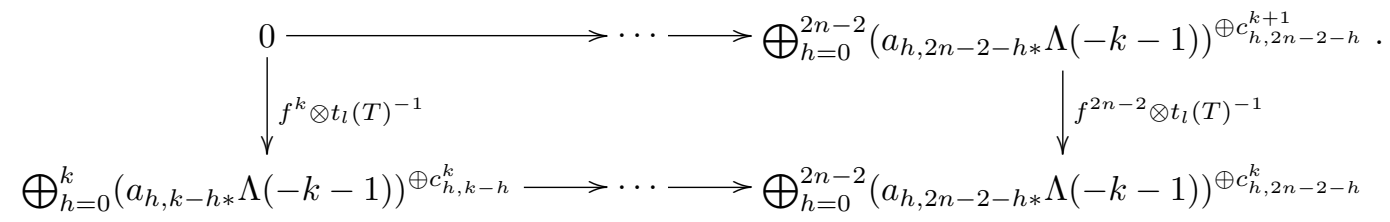

Let $\mathcal{K}=\operatorname{Cone}\left(f \otimes t_{l}(T)^{-1}: \mathcal{L}_{k+1} \rightarrow \mathcal{L}_{k}(-1)\right)$. The triangle

$$
R^{k} \psi \Lambda[-k-1] \longrightarrow i^{*} R^{k+1} j_{*} \Lambda[-k-1] \longrightarrow R^{k+1} \psi \Lambda[-k-1] \stackrel{\bar{N} \otimes t_{l}(T)^{-1}}{\longrightarrow} R^{k} \psi \Lambda[-k]
$$

is distinguished. It suffices to see that we can define a map $g: i^{*} R^{k+1} j_{*} \Lambda[-k] \rightarrow$ $\mathcal{K}$ which makes the first two squares of the following diagram commute:

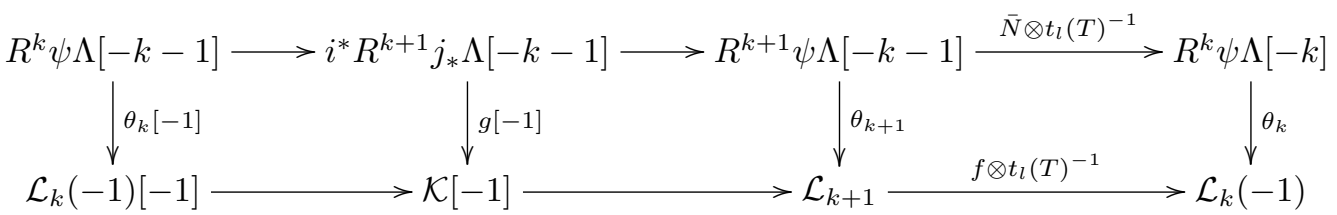

If the middle square is commutative, then there must exist $\theta^{\prime}: R^{k} \psi \Lambda[-k-1] \rightarrow$ $\mathcal{L}_{k}(-1)[-1]$ making the diagram a morphism of distinguished triangles. Then $\theta^{\prime}$ would make the first square commutative, so $\theta^{\prime}$ and $\theta_{k}[-1]$ coincide once they are pushed forward to $\mathcal{K}[-1]$. However,

$$
\operatorname{Hom}\left(R^{k} \psi \Lambda[-k-1], \mathcal{L}_{k+1}[-1]\right) \simeq \operatorname{Hom}\left(R^{k} \psi \Lambda[-k], R^{k+1} \psi \Lambda[-k-1]\right)=0
$$


so the Hom exact sequence associated to the bottom distinguished triangle implies that $\theta^{\prime}=\theta_{k}[-1]$. The diagram above is a morphism of distinguished triangles with $\theta_{k}[-1]$ as the leftmost morphism. This tells us that the third triangle in the diagram is also commutative, which is what we wanted to prove.

We can compute $i^{*} R^{k+1} j_{*} \Lambda$ using the $\log$ structure on $X$ :

$i^{*} R^{k+1} j_{*} \Lambda(k+1) \simeq \wedge^{k+1}\left(\left(\bigoplus_{j=1}^{n} a_{j *}^{1} \Lambda \oplus \bigoplus_{j=1}^{n} a_{j *}^{2} \Lambda\right) /(1, \ldots 1,0, \ldots 0)-(0, \ldots, 0,1, \ldots, 1)\right)$.

Here we have used, again, the formula $i^{*} R^{k} j_{*} \Lambda(k) \simeq \wedge^{k}\left(\bar{M}^{g p}\right) \otimes \Lambda$, which follows from Proposition 2.0.2 of [Na]. We can also compute $\mathcal{K}$ explicitly, since we have an explicit description of each $f^{k^{\prime}, h_{1}, h_{2}}$. The first non-zero term of $\mathcal{K}$ appears in degree $k$ and it is isomorphic to

$$
\sum_{h=0}^{k} a_{h, k-h *} \Lambda .
$$

There is a natural map of complexes $i^{*} R^{k+1} j_{*} \Lambda[-k] \rightarrow \mathcal{K}$, which sends

$$
a_{J_{1}, J_{2} *} \Lambda \rightarrow \bigoplus_{J_{1}^{\prime} \supset J_{1}, \# J_{1}^{\prime}=\# J_{1}+1} a_{J_{1}^{\prime}, J_{2} *} \Lambda \oplus \bigoplus_{J_{2}^{\prime} \supset J_{2}, \# J_{2}^{\prime}=\# J_{2}+1} a_{J_{1}, J_{2}^{\prime} *} \Lambda
$$

when $J_{1}, J_{2}$ are both non-empty. The map sends

$$
a_{J_{1}, \emptyset *} \Lambda \rightarrow \bigoplus_{\# J_{2}^{\prime}=1} a_{J_{1}, J_{2}^{\prime} *} \Lambda \text { and } a_{\emptyset, J_{2} *} \Lambda \rightarrow \bigoplus_{\# J_{1}^{\prime}=1} a_{J_{1}^{\prime}, J_{2} *} \Lambda
$$

It is easy to see that the above map is well-defined on $i^{*} R^{k+1} j_{*} \Lambda[-k]$ and that it is indeed a map of complexes. It remains to see that the above map of complexes $i^{*} R^{k+1} j_{*} \Lambda[-k] \rightarrow \mathcal{K}$ makes the first two squares of the diagram commute. This is tedious, but straightforward to verify.

Remark 4.3.5. Another way of proving the above corollary is to notice that 
Proposition 4.3.3 shows that the map

$$
\bar{N}: R^{k+1} \psi \Lambda[-k-1] \rightarrow R^{k} \psi \Lambda[-k]
$$

is given by the cup product with the map $\gamma \otimes t_{l}(T): \bar{M}_{r e l}^{g p}(-k-1) \rightarrow \Lambda(-k)[1]$ where $\gamma: \bar{M}_{r e l}^{g p} \rightarrow \Lambda[1]$ is the map corresponding to the class of the extension

$$
0 \rightarrow \Lambda \rightarrow \bar{M}^{g p} \rightarrow \bar{M}_{r e l}^{g p} \rightarrow 0
$$

of sheaves of $\Lambda$-modules on $Y$. Locally, $X$ is etale over a product of strictly semistable schemes $X_{1} \times_{\mathcal{O}_{K}} X_{2}$ and the extension $\bar{M}^{g p}$ is a Baire sum of extensions

$$
\begin{gathered}
0 \rightarrow \Lambda \rightarrow \bar{M}_{1}^{g p} \rightarrow \bar{M}_{1, \text { rel }}^{g p} \rightarrow 0 \text { and } \\
0 \rightarrow \Lambda \rightarrow \bar{M}_{2}^{g p} \rightarrow \bar{M}_{2, r e l}^{g p} \rightarrow 0
\end{gathered}
$$

which correspond to the $\log$ structures of $X_{1}$ and $X_{2}$ and which by Proposition 4.3.3 determine the maps $\bar{N}_{1}$ and $\bar{N}_{2}$. The Baire sum of extensions translates into $\bar{N}=\bar{N}_{1} \otimes 1+1 \otimes \bar{N}_{2}$ locally on $Y$. However, it is straightforward to check locally on $Y$ that the map $f: \mathcal{L}_{k} \rightarrow \mathcal{L}_{k+1}$ is the same as $\bar{N}_{1} \otimes 1+1 \otimes \bar{N}_{2}$. Thus, $f$ and $\bar{N}$ are maps of perverse sheaves on $Y$ which agree locally on $Y$, which means that $f$ and $\bar{N}$ agree globally. This proves the corollary without appealing to Proposition 4.3.3. (In fact, it suggests an alternate proof of Proposition 4.3.3.)

The following results, Lemma 4.3.6 to Corollary 4.3.10, are just generalizations of Lemma 4.2.8 to Corollary 4.2.13. We merely sketch their proofs here.

Lemma 4.3.6. The map $\bar{N}: R^{k} \psi \Lambda[-k] \rightarrow R^{k-1} \psi \Lambda[-k+1]$ has kernel

$$
\mathcal{P}_{k} \simeq\left[a_{k, k *} \Lambda(-k) \stackrel{\wedge \delta}{\rightarrow} a_{k, k+1 *} \Lambda(-k) \oplus a_{k+1, k *} \Lambda(-k) \rightarrow \cdots \stackrel{\wedge \delta}{\rightarrow} a_{n-1, n-1 *} \Lambda(-k)\right]
$$


where the first term is put in degree $2 k$ and cokernel

$$
\mathcal{R}_{k} \simeq\left[\oplus_{j=0}^{k-1} a_{j, k-1-j *} \Lambda(-(k-1)) \rightarrow \cdots \rightarrow a_{k-1, k-1 *} \Lambda((-(k-1))],\right.
$$

where the first term is put in degree $k-1$.

Proof. The proof is identical to the proof of Lemma 4.2.8, since by Proposition 4.3.3 we have a description of $\bar{N}$ as a degree by degree map

$$
f: \mathcal{L}_{k} \rightarrow \mathcal{L}_{k-1}
$$

Corollary 4.3.7. The filtration of $R \psi \Lambda$ by $\tau_{\leq k} R \psi \Lambda$ induces a filtration on ker $N$. The first graded piece of this filtration $\operatorname{Gr}^{1} \operatorname{ker} N$ is $R^{0} \psi \Lambda$. The graded piece $\mathrm{Gr}^{k+1}$ ker $N$ of this filtration is $\mathcal{P}_{k}$.

Proof. This can be proved the same way as Corollary 4.2.9. The only tricky part is seeing that we can identify a graded piece of ker $\bar{N}$ with a graded piece of ker $N$. In other words, we want to show that for $\bar{N}: R^{k} \psi \Lambda[-k] \rightarrow R^{k-1} \psi \Lambda[-k+$ 1] and $x \in \operatorname{ker} \bar{N}$ we can find a lift $\tilde{x} \in \tau_{\leq k} R \psi \Lambda$ of $x$ such that $\tilde{x} \in \operatorname{ker} N$. As in the proof of Corollary 4.2.9, we can define a map $\mathcal{P}_{k} \rightarrow \mathcal{R}_{k-1}$ sending $x$ to the image of $N \tilde{x}$ in $\mathcal{R}_{k-1}$, which turns out to be independent of the lift $\tilde{x}$. We want to see that this map vanishes but in fact any map $\mathcal{P}_{k} \rightarrow \mathcal{R}_{k-1}$ vanishes. Note that

$$
a_{h_{1}, h_{2} *} \Lambda\left[-h_{1}-h_{2}\right] \simeq \oplus_{\# S=h_{1}+1, \# T=h_{2}+1} a_{S, T *} \Lambda\left[-h_{1}-h_{2}\right] .
$$

The scheme $Y_{S, T}$ is smooth of pure dimension $\operatorname{dim} Y-h_{1}-h_{2}$ and so it is a disjoint union of its irreducible (connected) components which are smooth of pure dimension $\operatorname{dim} Y-h_{1}-h_{2}$. Thus, each $a_{S, T *} \Lambda\left[-h_{1}-h_{2}\right]$ is the direct 
sum of the pushforwards of the $-\operatorname{dim} Y$-shifted perverse sheaves $\Lambda\left[-h_{1}-h_{2}\right]$ on the irreducible components of $Y_{S, T}$. Thus, we have a decomposition of $a_{h_{1}, h_{2} *} \Lambda\left[-h_{1}-h_{2}\right]$ in terms of simple objects in the category of $-\operatorname{dim} Y$-shifted perverse sheaves. It is easy to check that $\mathcal{P}_{k}$ and $\mathcal{R}_{k-j}$ for $k \geq j \geq 1$ have no simple factors in common, so any map $\mathcal{P}_{k} \rightarrow \mathcal{R}_{k-j}$ must vanish.

Remark 4.3.8. The same techniques used in Section 4.2 apply in order to completely determine the graded pieces of $\left(\operatorname{ker} N^{j} / \operatorname{ker} N^{j-1}\right) /\left(\operatorname{im} N \cap \operatorname{ker} N^{j}\right)$ induced by the filtration of $R \psi \Lambda$ by $\tau_{\leq k} R \psi \Lambda$. The only tricky part is seeing that we can also identify the $k$ th graded piece of $i m N$ with

$$
\operatorname{im}\left(\bar{N}: R^{k+1} \psi \Lambda[-k-1] \rightarrow R^{k} \psi \Lambda[-k]\right)
$$

but this can be proved in the same way as the corresponding statement about the kernels of $N$ and $\bar{N}$. We get a complete description of the graded pieces of $\left(\operatorname{ker} N^{j} / \operatorname{ker} N^{j-1}\right) / \operatorname{im} N$.

Lemma 4.3.9. For $1 \leq j \leq 2 n-2$, the filtration of $R \psi \Lambda$ by $\tau_{\leq k} R \psi \Lambda$ induces a filtration on $\left(\operatorname{ker} N^{j} / \operatorname{ker} N^{j-1}\right) / \operatorname{im} N$. For $0 \leq k \leq n-1-\frac{j-1}{2}$, the $(k+1)$-st graded piece of this filtration is isomorphic to

$$
\oplus_{i=1}^{j} a_{k+i-1, k+j-i *} \Lambda(-(k+j-1))[-2 k-j+1] .
$$

As in Corollary 4.2.12, since each graded piece of the filtration is pure of weight $j-1$, the filtration is in fact a direct sum.

Let

$$
\operatorname{Gr}^{q} \operatorname{Gr}_{p} R \psi \Lambda=\left(\operatorname{ker} N^{p} \cap \operatorname{im} N^{q}\right) /\left(\operatorname{ker} N^{p-1} \cap \operatorname{im} N^{q}\right)+\left(\operatorname{ker} N^{p} \cap \operatorname{im} N^{q+1}\right) .
$$


The monodromy filtration $M_{r} R \psi \Lambda$ has graded pieces $\mathrm{Gr}_{r}^{M} R \psi \Lambda$ isomorphic to

$$
\mathrm{Gr}_{r}^{M} R \psi \Lambda \simeq \bigoplus_{p-q=r} \operatorname{Gr}^{q} \mathrm{Gr}_{p} R \psi \Lambda
$$

and if we understand the cohomology of $Y_{\overline{\mathbb{F}}}$ with coefficients in each $\mathrm{Gr}^{q} \mathrm{Gr}_{p} R \psi \Lambda$ we can compute the cohomology of $Y_{\overline{\mathbb{F}}}$ with respect to $R \psi \Lambda$. The next result tells us how to compute $H^{m}\left(Y_{\overline{\mathbb{F}}}, \mathrm{Gr}^{q} \mathrm{Gr}_{p} R \psi \Lambda\right)$.

Corollary 4.3.10. There is a direct sum decomposition

$H^{m}\left(Y_{\overline{\mathbb{F}}}, \operatorname{Gr}^{q} \operatorname{Gr}_{p} R \psi \Lambda\right) \simeq \bigoplus_{k \geq 0} \bigoplus_{i=1}^{p+q} H^{m}\left(Y_{\overline{\mathbb{F}}}, a_{k+i-1, k+p+q-i *} \Lambda(-(k+p-1))[-2 k-p-q+1]\right)$

compatible with the action of $G_{\overline{\mathbb{F}}}$. This can be rewritten as

$H^{m}\left(Y_{\overline{\mathbb{F}}}, \operatorname{Gr}^{q} \operatorname{Gr}_{p} R \psi \Lambda\right) \simeq \bigoplus_{k \geq 0} \bigoplus_{i=1}^{p+q} H^{m-2 k-p-q+1}\left(Y_{\overline{\mathbb{F}}}^{(k+i-1, k+p+q-i)}, \Lambda(-(k+p-1))\right)$.

Remark 4.3.11. The isomorphism above is functorial with respect to etale morphisms which preserve the stratification by $Y_{S, T}$ with $S, T \subset\{1, \ldots, n\}$. The reason for this is that etale morphisms preserve both the kernel and the image filtration of $N$ as well as the canonical filtration $\tau_{\leq k} R \psi \Lambda$. 


\section{Chapter 5}

\section{The cohomology of closed strata}

In this chapter we go back to working with the Iwahori level Shimura variety $X_{U} / \mathcal{O}_{K}$ as well as with the Shimura variety $X_{U_{0}} / \mathcal{O}_{K}$ with no level structure at $\mathfrak{p}_{1}$ and $\mathfrak{p}_{2}$, both corresponding to the unitary group $G$. Recall that $K=F_{\mathfrak{p}_{1}} \simeq$ $F_{\mathfrak{p}_{2}}$, with ring of integers $\mathcal{O}_{K}$, uniformizer $\pi$ and residue field $\mathbb{F}$.

\subsection{Igusa varieties}

Let $q=p^{\left[\mathbb{F}: \mathbb{F}_{p}\right]}$. Fix $0 \leq h_{1}, h_{2} \leq n-1$ and consider the stratum $\bar{X}_{U_{0}}^{\left(h_{1}, h_{2}\right)}$ of the Shimura variety $X_{U_{0}}$. Choose a compatible one-dimensional formal $\mathcal{O}_{F, \mathfrak{p}_{1}}=$ $\mathcal{O}_{K}$-module $\Sigma_{1}$, of height $n-h_{1}$ and also a compatible one-dimensional formal $\mathcal{O}_{F, \mathfrak{p}_{2}} \simeq \mathcal{O}_{K}$-module $\Sigma_{2}$ of height $n-h_{2}$. Giving $\Sigma_{1}$ and $\Sigma_{2}$ is equivalent to giving a triple $\left(\Sigma, \lambda_{\Sigma}, i_{\Sigma}\right)$ where:

- $\Sigma$ is a Barsotti-Tate group over $\overline{\mathbb{F}}$.

- $\lambda_{\Sigma}: \Sigma \rightarrow \Sigma^{\vee}$ is a polarization. 
- $i_{\Sigma}: \mathcal{O}_{F} \rightarrow \operatorname{End}(\Sigma) \otimes_{\mathbb{Z}} \mathbb{Z}_{(p)}$ such that $\left(\Sigma, i_{\Sigma}\right)$ is compatible.

Note that $\left(\Sigma\left[\mathfrak{p}_{i}^{\infty}\right]\right)^{0} \simeq \Sigma_{i}$ for $i=1,2$ while $\left(\Sigma\left[\mathfrak{p}_{i}^{\infty}\right]\right)^{\text {et }} \simeq\left(K / \mathcal{O}_{K}\right)^{n-h_{i}}$.

Assume that the level $U_{0}$ corresponds to the vector $\vec{m}=\left(0,0, m_{3}, \ldots, m_{r}\right)$. Let

$$
\overrightarrow{m^{\prime}}=\left(\left(m_{i}^{0}, m_{i}^{\mathrm{et}}\right)_{i=1,2}, m_{3}, \ldots, m_{r}\right)
$$

with the same entries $m_{3}, \ldots, m_{r}$ as $\vec{m}$. The Igusa variety $\operatorname{Ig}_{U^{p}, \vec{m}^{\prime}}^{\left(h_{1}, h_{2}\right)}$ over $\bar{X}_{U_{0}}^{\left(h_{1}, h_{2}\right)} \times_{\mathbb{F}}$ $\overline{\mathbb{F}}$ is defined to be the moduli space of the set of the following isomorphisms of finite flat group schemes for $i=1,2$ :

- $\alpha_{i}^{0}: \Sigma_{i}\left[\mathfrak{p}_{i}^{m_{i}^{0}}\right] \stackrel{\sim}{\rightarrow} \mathcal{G}_{i}^{0}\left[\mathfrak{p}_{i}^{m_{i}^{0}}\right]$, which extends etale locally to any $\left(m_{i}^{0}\right)^{\prime} \geq m_{i}^{0}$ and

$$
\text { - } \alpha_{i}^{\text {et }}:\left(\mathfrak{p}_{i}^{-m_{i}^{\text {et }}} \mathcal{O}_{F, \mathfrak{p}_{i}} / \mathcal{O}_{F, \mathfrak{p}_{i}}\right)^{h_{i}} \stackrel{\sim}{\rightarrow} \mathcal{G}_{i}^{\text {et }}\left[\mathfrak{p}_{i}^{m_{i}^{\text {et }}}\right] \text {. }
$$

In other words, if $S / \overline{\mathbb{F}}$ is a scheme, then an $S$-point of the Igusa variety $\operatorname{Ig}_{U^{p}, \vec{m}}^{\left(h_{1}, h_{2}\right)}$ corresponds to a tuple

$$
\left(A, \lambda, i, \eta^{p},\left(\alpha_{i}^{0}\right)_{i=1,2},\left(\alpha_{i}^{\mathrm{et}}\right)_{i=1,2},\left(\alpha_{i}\right)_{i \geq 3}\right),
$$

where

- $A$ is an abelian scheme over $S$ with $\mathcal{G}_{A, i}=A\left[\mathfrak{p}_{i}^{\infty}\right]$;

- $\lambda: A \rightarrow A^{\vee}$ is a prime-to- $p$ polarization;

- $i: \mathcal{O}_{F} \hookrightarrow \operatorname{End}(A) \otimes_{\mathbb{Z}} \mathbb{Z}_{(p)}$ such that $(A, i)$ is compatible and $\lambda \circ i(f)=$ $i\left(f^{*}\right)^{\vee} \circ \lambda, \forall f \in \mathcal{O}_{F}$

- $\bar{\eta}^{p}: V \otimes_{\mathbb{Q}} \mathbb{A}^{\infty, p} \rightarrow V^{p} A$ is a $\pi_{1}(S, s)$-invariant $U^{p}$-orbit of isomorphisms of $F \otimes_{\mathbb{Q}} \mathbb{A}^{\infty, p}$-modules, sending the standard pairing on $V \otimes_{\mathbb{Q}} \mathbb{A}^{\infty, p}$ to an $\left(\mathbb{A}^{\infty, p}\right)^{\times}$-multiple of the $\lambda$-Weil pairing; 
- $\alpha_{i}^{0}: \Sigma^{0}\left[\mathfrak{p}_{i}^{m_{i}^{0}}\right] \stackrel{\sim}{\rightarrow} \mathcal{G}_{A, i}^{0}\left[\mathfrak{p}_{i}^{m_{i}^{0}}\right]$ is an $\mathcal{O}_{K^{-}}$-equivariant isomorphism of finite flat group schemes which extends to any higher level $\left(m^{\prime}\right)_{i}^{0} \geq m_{i}^{0}$, for $i=1,2$ and some integer $\left(m^{\prime}\right)_{i}^{0}$;

- $\alpha_{i}^{\text {et }}: \Sigma^{\text {et }}\left[\mathfrak{p}_{i}^{m_{i}^{\mathrm{et}}}\right] \stackrel{\sim}{\rightarrow} \mathcal{G}_{A, i}^{\text {et }}\left[\mathfrak{p}_{i}^{m_{i}^{\text {et }}}\right]$ is an $\mathcal{O}_{K}$-equivariant isomorphism of etale group schemes, for $i=1,2$;

- $\alpha_{i}: \Sigma\left[\mathfrak{p}_{i}^{m_{i}}\right] \stackrel{\sim}{\rightarrow} \mathcal{G}_{A, i}\left[\mathfrak{p}_{i}^{m_{i}}\right]$ is an $\mathcal{O}_{F, \mathfrak{p}_{i}}$-equivariant isomorphism of etale group schemes, for $3 \leq i \leq r$.

Two such tuples are considered equivalent if there exists a prime-to- $p$ isogeny $f: A \rightarrow A^{\prime}$ taking $\left(A, \lambda, i, \bar{\eta}^{p}, \alpha_{i}^{0}, \alpha_{i}^{\mathrm{et}}, \alpha_{i}\right)$ to $\left(A^{\prime}, \gamma \lambda^{\prime}, i^{\prime}, \bar{\eta}^{p^{\prime}}, \alpha_{i}^{0^{\prime}}, \alpha_{i}^{\mathrm{et}^{\prime}}, \alpha_{i}^{\prime}\right)$ for $\gamma \in \mathbb{Z}_{(p)}^{\times}$

The Igusa varieties $\operatorname{Ig}_{U^{p}, \vec{m}}^{\left(h_{1}, h_{2}\right)}$ form an inverse system which has an action of $G\left(\mathbb{A}^{\infty, p}\right)$ inherited from the action on $\bar{X}_{U_{0}}^{\left(h_{1}, h_{2}\right)}$. Let

$J^{\left(h_{1}, h_{2}\right)}\left(\mathbb{Q}_{p}\right)=\mathbb{Q}_{p}^{\times} \times D_{K, n-h_{1}}^{\times} \times G L_{h_{1}}(K) \times D_{K, n-h_{2}}^{\times} \times G L_{h_{2}}(K) \times \prod_{i=3}^{r} G L_{n}\left(F_{\mathfrak{p}_{i}}\right)$,

which is the group of quasi-self-isogenies of $\left(\Sigma, \lambda_{\Sigma}, i_{\Sigma}\right)$ (to compute $J^{\left(h_{1}, h_{2}\right)}\left(\mathbb{Q}_{p}\right)$ we use the duality induced from the polarization). The automorphisms of $\left(\Sigma, \lambda_{\Sigma}, i_{\Sigma}\right)$ have an action on the right on $\operatorname{Ig}_{U^{p}, \vec{m}}^{\left(h_{1}, h_{2}\right)}$. This can be extended to an action of a certain submonoid of $J^{\left(h_{1}, h_{2}\right)}\left(\mathbb{Q}_{p}\right)$ on the inverse system of $\operatorname{Ig}_{U^{p}, \vec{m}}^{\left(h_{1}, h_{2}\right)}$ and furthermore to an action of the entire group $J^{\left(h_{1}, h_{2}\right)}\left(\mathbb{Q}_{p}\right)$ on the directed system $H_{c}^{j}\left(\operatorname{Ig}_{U^{p}, \vec{m}}^{\left(h_{1}, h_{2}\right)}, \mathcal{L}_{\xi}\right)$. For a definition of this action, see section 5 of [Sh1] and section 4 of [Man].

We also define an Iwahori-Igusa variety of the first kind $I_{U}^{\left(h_{1}, h_{2}\right)} / \bar{X}_{U_{0}}$ as the moduli space of chains of isogenies for $i=1,2$

$$
\mathcal{G}_{i}^{\mathrm{et}}=\mathcal{G}_{i, 0} \rightarrow \mathcal{G}_{i, 1} \rightarrow \cdots \rightarrow \mathcal{G}_{i, h_{i}}=\mathcal{G}_{i}^{\mathrm{et}} / \mathcal{G}_{i}^{\mathrm{et}}\left[\mathfrak{p}_{i}\right]
$$


of etale Barsotti-Tate $\mathcal{O}_{K}$-modules, each isogeny having degree $\# \mathbb{F}$ and with composite equal to the natural map $\mathcal{G}_{i}^{\text {et }} \rightarrow \mathcal{G}_{i}^{\text {et }} / \mathcal{G}_{i}^{\text {et }}\left[\mathfrak{p}_{i}\right]$. Then $I_{U}^{\left(h_{1}, h_{2}\right)}$ is finite etale over $\bar{X}_{U_{0}}^{\left(h_{1}, h_{2}\right)}$ and naturally inherits the action of $G\left(\mathbb{A}^{\infty, p}\right)$. Moreover, for $m_{1}^{0}=m_{2}^{0}=0$ and $m_{1}^{\text {et }}=m_{2}^{\text {et }}=1$ we know that $\operatorname{Ig}_{U^{p}, \vec{m}^{\prime}}^{\left(h_{1}, h_{2}\right)} / I_{U}^{\left(h_{1}, h_{2}\right)} \times_{\mathbb{F}} \overline{\mathbb{F}}$ is finite etale and Galois with Galois group $B_{h_{1}}(\mathbb{F}) \times B_{h_{2}}(\mathbb{F})$. (Here $B_{h_{i}}(\mathbb{F}) \subseteq G L_{h_{i}}(\mathbb{F})$ is the Borel subgroup.)

Lemma 5.1.1. For $S, T \subset\{1, \ldots, n\}$ with $\# S=n-h_{1}, \# T=n-h_{2}$ there exists a finite map of $\bar{X}_{U_{0}}^{\left(h_{1}, h_{2}\right)}$-schemes

$$
\varphi: Y_{U, S, T}^{0} \rightarrow I_{U}^{\left(h_{1}, h_{2}\right)}
$$

which is bijective on the sets of geometric points.

Proof. The proof is a straightforward generalization of the proof of Lemma 4.1 of $[\mathrm{TY}]$.

Recall that for a given $\vec{m}$ with $m_{1}=m_{2}=0$ we take

$$
U=U^{p} \times U_{p}^{\mathfrak{p}_{1}, \mathfrak{p}_{2}}(\vec{m}) \times \operatorname{Iw}_{n, \mathfrak{p}_{1}} \times \operatorname{Iw}_{n, \mathfrak{p}_{2}} \times \mathbb{Z}_{p}^{\times}
$$

restricting ourselves to Iwahori level structure at $\mathfrak{p}_{1}$ and $\mathfrak{p}_{2}$. Now we let the level away from $p$ vary. Define

$$
\begin{gathered}
H_{c}^{j}\left(Y_{\mathrm{Iw}(\vec{m}), S, T}^{0}, \mathcal{L}_{\xi}\right)=\lim _{U^{p}} H_{c}^{j}\left(Y_{U, S, T}^{0}, \mathcal{L}_{\xi}\right), \\
H^{j}\left(Y_{\mathrm{Iw}(\vec{m}), S, T}, \mathcal{L}_{\xi}\right)=\lim _{U^{p}} H^{j}\left(Y_{U, S, T}, \mathcal{L}_{\xi}\right), \\
H_{c}^{j}\left(I_{\operatorname{Iw}(\vec{m})}^{\left(h_{1}, h_{2}\right)}, \mathcal{L}_{\xi}\right)=\underset{\lim _{\overrightarrow{U^{p}}}}{ } H_{c}^{j}\left(I_{U}^{\left(h_{1}, h_{2}\right)} \times_{\mathbb{F}} \overline{\mathbb{F}}, \mathcal{L}_{\xi}\right) .
\end{gathered}
$$


Without restriction on $\vec{m}^{\prime}$ we can define

$$
H_{c}^{j}\left(\operatorname{Ig}^{\left(h_{1}, h_{2}\right)}, \mathcal{L}_{\xi}\right)=\underset{U^{p}, \vec{m}^{\prime}}{\lim } H_{c}^{j}\left(\operatorname{Ig}_{U^{p}, \vec{m}^{\prime}}^{\left(h_{1}, h_{2}\right)}, \mathcal{L}_{\xi}\right)
$$

For $m_{1}^{0}=m_{2}^{0}=0$, the Igusa variety $\operatorname{Ig}_{U^{p}, \vec{m}^{\prime}}^{\left(h_{1}, h_{2}\right)}$ is defined over $\mathbb{F}$. If in addition, $m_{1}^{\text {et }}=m_{2}^{\text {et }}=1$ then $\operatorname{Ig}_{U^{p}, \vec{m}^{\prime}}^{\left(h_{1}, h_{2}\right)}$ (over $\left.\mathbb{F}\right)$ is a Galois cover of $I_{U}^{\left(h_{1}, h_{2}\right)}$ with Galois group $B_{h_{1}}(\mathbb{F}) \times B_{h_{2}}(\mathbb{F})$.

Corollary 5.1.2. Let $\vec{m}^{\prime}=\left(0,0,1,1, m_{3}, \ldots, m_{r}\right)$. For every $S, T \subseteq\{1, \ldots n-$ 1\} with $\# S=n-h_{1}, \# T=n-h_{2}$ and $j \in \mathbb{Z}_{\geq 0}$ we have the following isomorphism

$$
H_{c}^{j}\left(Y_{U, S, T}^{0} \times_{\mathbb{F}} \overline{\mathbb{F}}, \mathcal{L}_{\xi}\right) \simeq H_{c}^{j}\left(I_{U^{p}, \vec{m}^{\prime}}^{\left(h_{1}, h_{2}\right)} \times_{\mathbb{F}} \overline{\mathbb{F}}, \mathcal{L}_{\xi}\right)^{B_{h_{1}}(\mathbb{F}) \times B_{h_{2}}(\mathbb{F})}
$$

By taking a direct limit over $U^{p}$ and over $\vec{m}=\left(0,0, m_{3}, \ldots, m_{r}\right)$ and considering the definitions of the Igusa varieties, we get an isomorphism

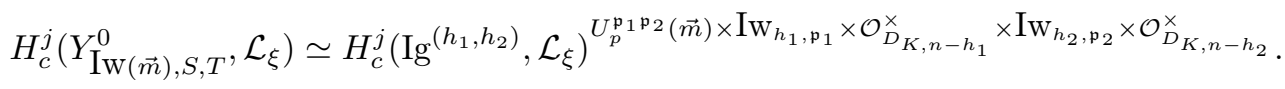

Taking a limit over general $\vec{m}^{\prime}$ satisfying $m_{1}^{0}=m_{2}^{0}=0$ we define

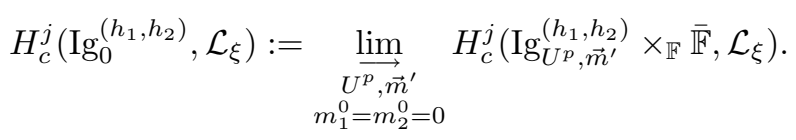

Then the above isomorphism becomes

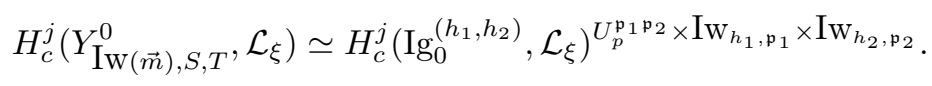

Proposition 5.1.3. The action of Frob $\mathrm{F}_{\mathbb{F}}$ on $H_{c}^{j}\left(\operatorname{Ig}_{0}^{\left(h_{1}, h_{2}\right)}, \mathcal{L}_{\xi}\right)$ coincides with the action of $\left(1,\left(p^{-\left[\mathbb{F}: \mathbb{F}_{p}\right]},-1,1,-1,1,1\right)\right) \in G\left(\mathbb{A}^{\infty, p}\right) \times J^{\left(h_{1}, h_{2}\right)}\left(\mathbb{Q}_{p}\right)$.

Proof. Let Fr : $x \mapsto x^{p}$ be the absolute Frobenius on $\mathbb{F}_{p}$ and let $f=\left[\mathbb{F}: \mathbb{F}_{p}\right]$. 
To compute the action of the geometric Frobenius Frob ${ }_{\mathbb{F}}$ on $H_{c}^{j}\left(\operatorname{Ig}_{0}^{\left(h_{1}, h_{2}\right)}, \mathcal{L}_{\xi}\right)$ we notice that the absolute Frobenius acts on each $H_{c}^{j}\left(\operatorname{Ig}_{U^{p}, \vec{m}}^{\left(h_{1}, h_{2}\right)} \times_{\mathbb{F}} \overline{\mathbb{F}}, \mathcal{L}_{\xi}\right)$ as $\left(\mathrm{Fr}^{*}\right)^{f} \times\left(\mathrm{Frob}_{\mathbb{F}}^{*}\right)^{-1}$. However, the absolute Frobenius acts trivially on etale cohomology, so the action of Frob $\mathbb{F}_{\mathbb{F}}$ coincides with the action induced from

$$
\left(\operatorname{Fr}^{*}\right)^{f}: \operatorname{Ig}_{U^{p}, \vec{m}^{\prime}}^{\left(h_{1}, h_{2}\right)} \rightarrow \operatorname{Ig}_{U^{p}, \vec{m}^{\prime}}^{\left(h_{1}, h_{2}\right)}
$$

We claim that $\left(\operatorname{Fr}^{*}\right)^{f}$ acts the same as the element $\left(1, p^{-\left[\mathbb{F}: \mathbb{F}_{p}\right]},-1,1,-1,1,1\right)$ of

$$
G\left(\mathbb{A}^{\infty, p}\right) \times \mathbb{Q}_{p}^{\times} / \mathbb{Z}_{p}^{\times} \times \mathbb{Z} \times G L_{h_{1}}(K) \times \mathbb{Z} \times G L_{h_{2}}(K) \times \prod_{i=3}^{r} G L_{n}\left(F_{\mathfrak{p}_{i}}\right),
$$

where the two copies of $\mathbb{Z}$ are identified with $D_{K, n-h_{i}}^{\times} / \mathcal{O}_{D_{K, n-h_{i}}}^{\times}$for $i=1,2$ via the valuation of the determinant. To verify this claim, we will use the explicit description of the action of a submonoid $J^{\left(h_{1}, h_{2}\right)}\left(\mathbb{Q}_{p}\right)$ on the inverse system of Igusa varieties $\operatorname{Ig}_{U^{p}, \vec{m}^{\prime}}^{\left(h_{1}, h_{2}\right)}$ found in [Man] which generalizes that on page 122 of $[\mathrm{HT}]$. First, it is easy to see that

$\left(\operatorname{Fr}^{*}\right)^{f}:\left(A, \lambda, i, \bar{\eta}^{p}, \alpha_{i}^{0}, \alpha_{i}^{\mathrm{et}}, \alpha_{i}\right) \mapsto\left(A^{(q)}, \lambda^{(q)}, i^{(q)},\left(\bar{\eta}^{p}\right)^{(q)},\left(\alpha_{i}^{0}\right)^{(q)},\left(\alpha_{i}^{\mathrm{et}}\right)^{(q)}, \alpha_{i}^{(q)}\right)$

where $F^{f}: A \rightarrow A^{(q)}$ is the natural map and the structures of $A^{(q)}$ are inherited from the structures of $A$ via $F^{f}$.

On the other hand, the element $j=\left(1, p^{-\left[\mathbb{F}: \mathbb{F}_{p}\right]},-1,1,-1,1,1\right)$ acts via a quasi-isogeny of $\Sigma$. One can check that the inverse of the quasi-isogeny defined by $j$ is $j^{-1}: \Sigma \rightarrow \Sigma^{(q)}$, which is a genuine isogeny. If we were working with points of $\operatorname{Ig}^{\left(h_{1}, h_{2}\right)}$ (which are compatible systems of points of $\operatorname{Ig}_{U^{p}, \vec{m}^{\prime}}^{\left(h_{1}, h_{2}\right)}$ for all $U^{p}$ and $\vec{m}^{\prime}$ ) then $j$ should act by precomposing all the isomorphism $\alpha_{i}^{0}, \alpha_{i}^{\text {et }}$ for $i=1,2$ and $\alpha_{i}$ for $3 \leq i \leq r$. Since $\left.j\right|_{A\left[\mathfrak{p}_{i}^{\infty}\right]}$ et $=1$ for $i=1,2$ and $\left.j\right|_{A\left[\mathfrak{p}_{i}^{\infty}\right]}=1$ for $3 \leq i \leq r$ the isomorphisms $\alpha_{i}^{\text {et }}$ and $\alpha_{i}$ stay the same. However, $\alpha_{i}^{0} \circ j$ is now only 
a quasi-isogeny of Barsotti-Tate $\mathcal{O}_{K}$-modules and we need to change the abelian variety $A$ by an isogeny in order to get back the isomorphisms. Let $j_{i}=\left.j\right|_{\Sigma\left[\mathfrak{p}_{i}^{\infty}\right]^{0}}$ for $i=1,2$. Then $\left(j_{i}\right)^{-1}: \Sigma\left[\mathfrak{p}_{i}^{\infty}\right]^{0} \rightarrow \Sigma\left[\mathfrak{p}_{i}^{\infty}\right]^{0}$ is a genuine isogeny induced by the action of $\pi_{i} \in D_{K, n-h_{i}}^{\times}$. Let $\mathcal{K}_{i} \subset A\left[\mathfrak{p}_{i}^{\left[\mathbb{F}: \mathbb{F}_{p}\right]}\right]$ be the finite flat subgroup scheme $\alpha_{i}^{0}\left(\operatorname{ker}\left(j_{i}\right)^{-1}\right)$. Let $\mathcal{K}=\mathcal{K}_{1} \oplus \mathcal{K}_{2} \subset A\left[u^{\left[\mathbb{F}: \mathbb{F}_{p}\right]}\right]$. Let $\mathcal{K}^{\perp} \subset A\left[\left(u^{c}\right)^{\left[\mathbb{F}: \mathbb{F}_{p}\right]}\right]$ be the annihilator of $\mathcal{K}$ under the $\lambda$-Weil pairing. Let $\tilde{A}=A / \mathcal{K} \oplus \mathcal{K}^{\perp}$ and $f: A \rightarrow \tilde{A}$ be the natural projection map. Then

$$
\beta_{i}^{0}=f \circ \alpha_{i}^{0} \circ j_{i}:\left(\Sigma\left[\mathfrak{p}_{i}^{\infty}\right]\right)^{0} \rightarrow \tilde{A}\left[\mathfrak{p}_{i}^{\infty}\right]^{0}
$$

is an isomorphism. The quotient abelian variety $\tilde{A}$ inherits the structures of $A$ through the natural projection and it is easy to see that $\tilde{A}=A^{(q)}$. Thus, the action of $j$ coincides with the action of $\left(\mathrm{Fr}^{*}\right)^{f}$. This concludes the proof.

Corollary 5.1.4. We have an isomorphism of admissible $G\left(\mathbb{A}^{\infty, p}\right) \times\left(\operatorname{Frob}_{\mathbb{F}}\right)^{\mathbb{Z}}$ modules

$$
H_{c}^{j}\left(Y_{\mathrm{Iw}(\vec{m}), S, T}^{0}, \mathcal{L}_{\xi}\right) \simeq H_{c}^{j}\left(\operatorname{Ig}_{0}^{\left(h_{1}, h_{2}\right)}, \mathcal{L}_{\xi}\right)^{U_{p}^{\mathfrak{p}_{1} \mathfrak{p}_{2}}(\vec{m}) \times \operatorname{Iw}_{h_{1}, \mathfrak{p}_{1}} \times \operatorname{Iw}_{h_{2}, \mathfrak{p}_{2}}}
$$

where Frob $b_{\mathbb{F}}$ acts as $\left(p^{-f},-1,1,-1,1,1\right) \in J^{\left(h_{1}, h_{2}\right)}\left(\mathbb{Q}_{p}\right)$.

\subsection{Counting points on Igusa varieties}

We wish to apply the trace formula in order to compute the cohomology of Igusa varieties. A key input of this is counting the $\overline{\mathbb{F}}$-points of Igusa varieties. Most of this is worked out in [Sh1]. The only missing ingredient is supplied by the main lemma in this section, which is an analogue of Lemma V.4.1 in [HT] and of "the vanishing of the Kottwitz invariant". The $\overline{\mathbb{F}}$-points of Igusa varieties are counted by counting $p$-adic types and other data (e.g polarizations and level 
structure). We can keep track of $p$-adic types via Honda-Tate theory; we need to check that these $p$-adic types actually correspond to a point on one of our Igusa varieties.

A simple $p$-adic type over $F$ is a triple $(M, \eta, \kappa)$ where

- $M$ is a CM field, with $\mathfrak{P}$ being the set of places of $M$ over $p$,

- $\eta=\sum_{x \in \mathfrak{P}} \eta_{x} x$ is an element of $\mathbb{Q}[\mathfrak{P}]$, the $\mathbb{Q}$-vector space with basis $\mathfrak{P}$,

- $\kappa: F \rightarrow M$ is a $\mathbb{Q}$-algebra homomorphism

such that $\eta_{x} \geq 0$ for all $x \in \mathfrak{P}$ and $\eta+c_{*} \eta=\sum_{x \in \mathfrak{P}} x(p) \cdot x$ in $\mathbb{Q}[\mathfrak{P}]$, where $p=\prod_{x \in \mathfrak{P}} x^{x(p)}$. Here $c$ is the complex conjugation on $M$ and

$$
c_{*}: \mathbb{Q}[\mathfrak{P}] \rightarrow \mathbb{Q}[\mathfrak{P}]
$$

is the $\mathbb{Q}$-linear map satisfying $x \mapsto x^{c}$. See page 24 of [Sh1] for the general definition of a $p$-adic type. As in [Sh1], we will drop $\kappa$ from the notation, since it is well understood as the $F$-algebra structure map of $M$.

We can recover a simple $p$-adic type from the following data:

- a CM field $M / F$;

- for $i=1,2$ places $\tilde{\mathfrak{p}}_{i}$ of $M$ above $\mathfrak{p}_{i}$ such that $\left[M_{\tilde{\mathfrak{p}}_{i}}: F_{\mathfrak{p}_{i}}\right] n=[M: F]\left(n-h_{i}\right)$ and such that there is no intermediate field $F \subset N \subset M$ with $\left.\tilde{\mathfrak{p}}_{i}\right|_{N}$ both inert in $M$.

Using this data, we can define a simple $p$-adic type $(M, \eta)$, where the coefficients of $\eta$ at places above $u$ are non-zero only for $\tilde{\mathfrak{p}}_{1}$ and $\tilde{\mathfrak{p}}_{2}$. The abelian variety $A / \overline{\mathbb{F}}$ corresponding to $(M, \eta)$ will have an action of $M$ via $i: M \hookrightarrow \operatorname{End}^{0}(A)$. By Honda-Tate theory, the pair $(A, i)$ will also satisfy

- $M$ is the center of $\operatorname{End}_{F}^{0}(A)$, 
- $A\left[\mathfrak{p}_{i}^{\infty}\right]^{0}=A\left[\tilde{\mathfrak{p}}_{i}^{\infty}\right]$ has dimension 1 and $A\left[\mathfrak{p}_{i}^{\infty}\right]^{e}$ has height $h_{i}$ for $i=1,2$,

- and $A\left[\mathfrak{p}_{i}^{\infty}\right]$ is ind-etale for $i>2$.

Lemma 5.2.1. Let $M / F$ be a $C M$ field as above. Let $A / \overline{\mathbb{F}}$ be the corresponding abelian variety equipped with $i: M \hookrightarrow E n d^{0}(A)$. Then we can find

- a polarization $\lambda_{0}: A \rightarrow A^{\vee}$ for which the Rosati involution induces $c$ on $i(M)$, and

- a finitely-generated $M$-module $W_{0}$ together with a non-degenerate Hermitian pairing

$$
\langle\cdot, \cdot\rangle_{0}: W_{0} \times W_{0} \rightarrow \mathbb{Q}
$$

such that the following are satisfied:

- there is an isomorphism of $M \otimes \mathbb{A}^{\infty, p}$-modules

$$
W_{0} \otimes \mathbb{A}^{\infty, p} \stackrel{\sim}{\rightarrow} V^{p} A
$$

which takes $\langle\cdot, \cdot\rangle_{0}$ to an $\left(\mathbb{A}^{\infty, p}\right)^{\times}$-multiple of the $\lambda_{0}$-Weil pairing on $V^{p} A$, and

- there is an isomorphism of $F \otimes_{\mathbb{Q}} \mathbb{R}$-modules

$$
W_{0} \otimes_{\mathbb{Q}} \mathbb{R} \stackrel{\sim}{\rightarrow} V \otimes_{\mathbb{Q}} \mathbb{R}
$$

which takes $\langle\cdot, \cdot\rangle_{0}$ to an $\mathbb{R}^{\times}$-multiple of our standard pairing $\langle\cdot, \cdot\rangle$ on $V \otimes_{\mathbb{Q}}$ $\mathbb{R}$.

Proof. By Lemma 9.2 of [Ko1] there is a polarization $\lambda_{0}: A \rightarrow A^{\vee}$ such that the $\lambda_{0}$-Rosati involution preserves $M$ and acts on it as $c$. The next step is to show that, up to isogeny, we can lift $\left(A, i, \lambda_{0}\right)$ from $\overline{\mathbb{F}}$ to $\mathcal{O}_{K^{a c}}$. Using the results 
of [Tat] we can find some lift of $A$ to an abelian scheme $\tilde{A} / \mathcal{O}_{K^{a c}}$ in such a way that $i$ lifts to an action $\tilde{i}$ of $M$ on $\tilde{A}$. As in the proof of Lemma V.4.1 of [HT] we find a polarization $\tilde{\lambda}$ of $\tilde{A}$ which reduces to $\lambda$. However, we want to be more specific about choosing our lift $\tilde{A}$. Indeed, for any lift, $\operatorname{Lie} \tilde{A} \otimes_{\mathcal{O}_{K}^{a c}} K^{a c}$ is an $F \otimes K^{a c} \simeq\left(K^{a c}\right)^{\operatorname{Hom}\left(F, K^{a c}\right)}$-module, so we have a decomposition

$$
\operatorname{Lie} \tilde{A} \otimes_{\mathcal{O}_{K^{a c}}} K^{a c} \simeq \bigoplus_{\tau \in \operatorname{Hom}\left(F, K^{a c}\right)}(\operatorname{Lie} \tilde{A})_{\tau}
$$

Let $\operatorname{Hom}\left(F, K^{a c}\right)^{+}$be the the set of places $\tau \in \operatorname{Hom}\left(F, K^{a c}\right)$ which induce the place $u$ of $E$. We want to make sure that the set of places $\tau \in \operatorname{Hom}\left(F, K^{a c}\right)^{+}$ for which $(\operatorname{Lie} \tilde{A})_{\tau}$ is non-trivial has exactly two elements $\tau_{1}^{\prime}$ and $\tau_{2}^{\prime}$ which differ by our distinguished element $\sigma \in \operatorname{Gal}(F / \mathbb{Q})$, i.e.

$$
\tau_{2}^{\prime}=\tau_{1}^{\prime} \circ \sigma
$$

In order to ensure this, we need to go through Tate's original argument for constructing lifts $\tilde{A}$ of $A$.

First, let $\Phi=\sum_{\tilde{\tau} \in \operatorname{Hom}\left(M, K^{a c}\right)} \Phi_{\tilde{\tau}} \cdot \tilde{\tau}$ with the $\Phi_{\tilde{\tau}}$ non-negative integers satisfying $\Phi_{\tilde{\tau}}+\Phi_{\tilde{\tau}^{c}}=n$. For any such $\Phi$, we can construct an abelian variety $\tilde{A}_{\Phi}$ over $\mathcal{O}_{K^{a c}}$ such that

$$
\operatorname{Lie} \tilde{A}_{\Phi} \otimes_{\mathcal{O}_{K^{a c}}} K^{a c} \simeq \bigoplus_{\tau \in \operatorname{Hom}\left(F, K^{a c}\right)}\left(\operatorname{Lie} \tilde{A}_{\Phi}\right)_{\tau}
$$

satisfies $\operatorname{dim}\left(\operatorname{Lie} \tilde{A}_{\Phi}\right)_{\tau}=\Phi_{\tau}$. This is done as in Lemma 4 of [Tat], which proves the case $n=1$. We pick any $\tau_{i}^{\prime} \in \operatorname{Hom}\left(F, K^{a c}\right)$ inducing the places $\mathfrak{p}_{i}$ of $F$ for $i=$ 1,2 such that $\tau_{2}^{\prime}=\tau_{1}^{\prime} \circ \sigma$. We lift the $\tau_{i}^{\prime}$ to elements $\tilde{\tau}_{i} \in \operatorname{Hom}\left(M, K^{a c}\right)$ inducing $\tilde{\mathfrak{p}}_{i}$. We let $\Phi_{\tilde{\tau}_{i}}=1$ and $\Phi_{\tilde{\tau}}=0$ for any other $\tilde{\tau} \in \operatorname{Hom}\left(M, K^{a c}\right)^{+}$. For $\tilde{\tau} \notin$ $\operatorname{Hom}\left(M, K^{a c}\right)^{+}$we define $\Phi_{\tilde{\tau}}=n-\Phi_{\tilde{\tau}^{c}}$. This determines $\Phi \in \mathbb{Q}\left[\operatorname{Hom}\left(M, K^{a c}\right)\right]$ 
entirely. This $\Phi$ is not quite a $p$-adic type for $M$, however it is easy to associate a $p$-adic type to it: we define $\eta=\sum_{x \mid p} \eta_{x} \cdot x$ by

$$
\eta_{x}=\frac{e_{x / p} \cdot[M: F]}{n \cdot\left[M_{x}: \mathbb{Q}_{p}\right]} \cdot \sum \Phi_{\tilde{\tau}}
$$

where the sum is over embeddings $\tilde{\tau} \in \operatorname{Hom}\left(M, K^{a c}\right)$ which induce the place $x$ of $M$. By Honda-Tate theory, the reduction of the abelian scheme $\tilde{A}_{\Phi} / \mathcal{O}_{K^{a c}}$ associated to $\Phi$ has $p$-adic type $\eta$. Indeed, the height of the $p$-divisible group at $x$ of the reduction of $\tilde{A}_{\Phi}$ is $\frac{n \cdot\left[M_{x}: \mathbb{Q}_{p}\right]}{[M: F]}$ (see Proposition 8.4 of [Sh1] together with an expression of $\operatorname{dim} A$ in terms of $M)$. The dimension of the $p$-divisible group at $x$ of the reduction is $\sum \Phi_{\tilde{\tau}}$, where we're summing over all embeddings $\tilde{\tau}$ which induce $x$.

Now we set $\tilde{A}=\tilde{A}_{\Phi}$. It remains to check that $\tilde{A} / \mathcal{O}_{K^{a c}}$ has special fiber isogenous to $A / \overline{\mathbb{F}}$ and this follows from the fact that the reductions of $\tilde{A}$ and $A$ are both associated to the same $p$-adic type $\eta$. Indeed, it suffices to verify this for places $x$ above $u$. We have

$$
\eta_{x}=0=e_{x / p} \cdot \frac{\operatorname{dim} A\left[x^{\infty}\right]}{\operatorname{height} A\left[x^{\infty}\right]}
$$

for all places $x \neq \tilde{\mathfrak{p}}_{i}$ for $i=1,2$. When $x=\tilde{\mathfrak{p}}_{i}$ we have

$\eta_{x}=e_{x / p} \cdot \frac{[M: F]}{\left[M_{x}: F_{\mathfrak{p}_{i}}\right] \cdot n \cdot\left[F_{\mathfrak{p}_{i}}: \mathbb{Q}_{p}\right]}=e_{x / p} \cdot \frac{1}{\left(n-h_{i}\right) \cdot\left[F_{\mathfrak{p}_{i}}: \mathbb{Q}_{p}\right]}=e_{x / p} \cdot \frac{\operatorname{dim} A\left[x^{\infty}\right]}{\operatorname{height} A\left[x^{\infty}\right]}$

Therefore, the $p$-adic type associated to $A$ is also $\eta$.

There are exactly two distinct embeddings $\tau_{1}^{\prime}, \tau_{2}^{\prime} \in \operatorname{Hom}\left(F, K^{a c}\right)^{+}$such that $(\operatorname{Lie} \tilde{A})_{\tau} \neq(0)$ only when $\tau=\tau_{1}^{\prime}$ or $\tau_{2}^{\prime}$. Moreover, these embeddings are related by $\tau_{2}^{\prime}=\tau_{1}^{\prime} \circ \sigma$. Therefore, we can find an embedding $\kappa: K^{a c} \hookrightarrow \mathbb{C}$ such that 
$\kappa \circ \tau_{i}^{\prime}=\tau_{i}$ for $i=1,2$. We set

$$
W_{0}=H_{1}\left(\left(\tilde{A} \times \operatorname{Spec} \mathcal{O}_{K^{a c}, \kappa} \operatorname{Spec} \mathbb{C}\right)(\mathbb{C}), \mathbb{Q}\right) .
$$

From here on, the proof proceeds as the proof of Lemma V.4.1 of [HT].

\subsection{Vanishing of cohomology}

Let $\Pi^{1}$ be an automorphic representation of $G L_{1}\left(\mathbb{A}_{E}\right) \times G L_{n}\left(\mathbb{A}_{F}\right)$ and assume that $\Pi^{1}$ is cuspidal. Let $\varpi: \mathbb{A}_{E}^{\times} / E^{\times} \rightarrow \mathbb{C}$ be any Hecke character such that $\left.\varpi\right|_{\mathbb{A}^{\times} / \mathbb{Q}^{\times}}$is the composite of $\operatorname{Art}_{\mathbb{Q}}$ and the natural surjective character $W_{\mathbb{Q}} \rightarrow$ $\operatorname{Gal}(E / \mathbb{Q}) \stackrel{\sim}{\rightarrow}\{ \pm 1\}$.

Also assume that $\Pi^{1}$ and $F$ satisfy

- $\Pi^{1} \simeq \Pi^{1} \circ \theta$.

- $\Pi_{\infty}^{1}$ is generic and $\Xi^{1}$-cohomological, for some irreducible algebraic representation $\Xi^{1}$ of $\mathbb{G}_{n}(\mathbb{C})$, which is the image of $\iota_{l} \xi$ under the base change from $G_{\mathbb{C}}$ to $\mathbb{G}_{n, \mathbb{C}}$.

- $\operatorname{Ram}_{F / \mathbb{Q}} \cup \operatorname{Ram}_{\mathbb{Q}}(\varpi) \cup \operatorname{Ram}_{\mathbb{Q}}(\Pi) \subset \operatorname{Spl}_{F / F_{2}, \mathbb{Q}}$.

Let $\mathfrak{S}=\mathfrak{S}_{\text {fin }} \cup\{\infty\}$ be a finite set of places of $F$, which contains the places of $F$ above places of $\mathbb{Q}$ which are ramified in $F$ and the places where $\Pi$ is ramified. For $l \neq p$, let $\iota: \overline{\mathbb{Q}}_{l} \stackrel{\sim}{\rightarrow} \mathbb{C}$ and let $\pi_{p} \in \operatorname{Irr}_{l}\left(G\left(\mathbb{Q}_{p}\right)\right)$ be such that $B C\left(\iota_{l} \pi_{p}\right) \simeq \Pi_{p}$. If we write $\Pi^{1}=\psi \otimes \Pi^{0}$ and $\pi_{p}=\pi_{p, 0} \otimes \pi_{\mathfrak{p}_{1}} \otimes \pi_{\mathfrak{p}_{2}} \otimes\left(\otimes_{i=3}^{r} \pi_{\mathfrak{p}_{i}}\right)$ then $\iota_{l} \pi_{p, 0} \simeq \psi_{u}$ and $\iota_{l} \pi_{\mathfrak{p}_{i}} \simeq \Pi_{\mathfrak{p}_{i}}^{0}$ for all $1 \leq i \leq r$. Under the identification $F_{\mathfrak{p}_{1}} \simeq F_{\mathfrak{p}_{2}}$, assume that $\Pi_{\mathfrak{p}_{1}}^{0} \simeq \Pi_{\mathfrak{p}_{2}}^{0}$ (this condition will be satisfied in all our applications, since we will choose $\Pi^{0}$ to be the base change of some cuspidal automorphic representation $\Pi$ of $\left.G L_{n}\left(\mathbb{A}_{F_{1} E}\right)\right)$. 
Define the following elements of $\operatorname{Groth}\left(G\left(\mathbb{A}^{\infty, p}\right) \times J^{\left(h_{1}, h_{2}\right)}\left(\mathbb{Q}_{p}\right)\right)$ (the Grothendieck group of admissible representations):

$$
\left[H_{c}\left(\operatorname{Ig}^{\left(h_{1}, h_{2}\right)}, \mathcal{L}_{\xi}\right)\right]=\sum_{i}(-1)^{h_{1}+h_{2}-i} H_{c}^{i}\left(\operatorname{Ig}^{\left(h_{1}, h_{2}\right)}, \mathcal{L}_{\xi}\right)
$$

If $R \in \operatorname{Groth}\left(G\left(\mathbb{A}^{\mathfrak{S}}\right) \times G^{\prime}\right)$, we can write $R=\sum_{\pi^{\mathfrak{S}} \otimes \rho} n\left(\pi^{\mathfrak{S}} \otimes \rho\right)\left[\pi^{\mathfrak{S}}\right][\rho]$, where $\pi^{\mathfrak{S}}$ and $\rho$ run over $\operatorname{Irr}_{l}\left(G\left(\mathbb{A}^{\mathfrak{S}}\right)\right)$ and $\operatorname{Irr}_{l}\left(G^{\prime}\right)$ respectively. We define

$$
R\left\{\pi^{\mathfrak{S}}\right\}:=\sum_{\rho} n\left(\pi^{\mathfrak{S}} \otimes \rho\right)[\rho], R\left[\pi^{\mathfrak{S}}\right]:=\sum_{\rho} n\left(\pi^{\mathfrak{S}} \otimes \rho\right)\left[\pi^{\mathfrak{S}}\right][\rho] .
$$

Also define

$$
R\left\{\Pi^{1, \mathfrak{S}}\right\}:=\sum_{\pi^{\mathfrak{S}}}\left\{\pi^{\mathfrak{S}}\right\}, R\left[\Pi^{1, \mathfrak{S}}\right]:=\sum_{\pi^{\mathfrak{S}}} R\left[\pi^{\mathfrak{S}}\right],
$$

where each sum runs over $\pi^{\mathfrak{S}} \in \operatorname{Irr}_{l}^{\mathrm{ur}}\left(G\left(\mathbb{A}^{\mathfrak{S}}\right)\right)$ such that $B C\left(\iota_{l} \pi^{\mathfrak{S}}\right) \simeq \Pi^{\mathfrak{S}}$.

Let $\operatorname{Red}_{n}^{\left(h_{1}, h_{2}\right)}\left(\pi_{p}\right)$ be the morphism from $\operatorname{Groth}\left(G\left(\mathbb{Q}_{p}\right)\right)$ to $\operatorname{Groth}\left(J^{\left(h_{1}, h_{2}\right)}\left(\mathbb{Q}_{p}\right)\right)$ defined by

$$
(-1)^{h_{1}+h_{2}} \pi_{p, 0} \otimes \operatorname{Red}^{n-h_{1}, h_{1}}\left(\pi_{\mathfrak{p}_{1}}\right) \otimes \operatorname{Red}^{n-h_{2}, h_{2}}\left(\pi_{\mathfrak{p}_{2}}\right) \otimes\left(\otimes_{i>2} \pi_{\mathfrak{p}_{i}}\right),
$$

where

$$
\operatorname{Red}^{n-h, h}: \operatorname{Groth}\left(G L_{n}(K)\right) \rightarrow \operatorname{Groth}\left(D_{K, \frac{1}{n-h}}^{\times} \times G L_{h}(K)\right)
$$

is obtained by composing the normalized Jacquet functor

$$
J: \operatorname{Groth}\left(G L_{n}(K)\right) \rightarrow \operatorname{Groth}\left(G L_{n-h}(K) \times G L_{h}(K)\right)
$$


with the Jacquet-Langlands map

$$
L J: \operatorname{Groth}\left(G L_{n-h}(K)\right) \rightarrow \operatorname{Groth}\left(D_{K, \frac{1}{n-h}}^{\times}\right)
$$

defined by Badulescu in [Bad]. Assume the following result, which will be proved in section 6 :

Theorem 5.3.1. We have the following equality in $\operatorname{Groth}\left(G\left(\mathbb{A}_{\mathfrak{S}_{f i n} \backslash\{p\}}\right) \times J^{\left(h_{1}, h_{2}\right)}\left(\mathbb{Q}_{p}\right)\right.$ :

$$
\begin{gathered}
B C_{\mathfrak{S}_{f i n} \backslash\{p\}}\left(H_{c}\left(\operatorname{Ig}^{\left(h_{1}, h_{2}\right)}, \mathcal{L}_{\xi}\right)\left\{\Pi^{1, \mathfrak{S}}\right\}\right) \\
=e_{0}(-1)^{h_{1}+h_{2}} C_{G}\left[\iota_{l}^{-1} \Pi_{\mathfrak{S}_{f i n} \backslash\{p\}}^{1}\right]\left[\operatorname{Red}_{n}^{\left(h_{1}, h_{2}\right)}\left(\pi_{p}\right)\right],
\end{gathered}
$$

where $C_{G}$ is a positive integer and $e_{0}= \pm 1$.

Let $S, T \subseteq\{1, \ldots, n-1\}$ with $\# S=n-h_{1}, \# T=n-h_{2}$. From Theorem 5.3.1 and Corollary 5.1.4 we obtain the equality

$$
\begin{gathered}
B C^{p}\left(H_{c}\left(Y_{\mathrm{Iw}(m), S, T}^{0}, \mathcal{L}_{\xi}\right)\left[\Pi^{1, \mathfrak{S}}\right]\right) \\
=e_{0} C_{G}\left[\iota_{l}^{-1} \Pi^{\infty, p}\right]\left[\operatorname{Red}^{\left(h_{1}, h_{2}\right)}\left(\pi_{p, 0} \otimes \pi_{\mathfrak{p}_{1}} \otimes \pi_{\mathfrak{p}_{2}}\right)\right] \cdot \operatorname{dim}\left[\left(\otimes_{i=3}^{r} \pi_{\mathfrak{p}_{i}}\right)^{U_{p}^{\mathfrak{p}_{1} \mathfrak{p}_{2}}}\right]
\end{gathered}
$$

in $\operatorname{Groth}\left(G\left(\mathbb{A}^{\infty, p}\right) \times\left(\operatorname{Frob}_{\mathbb{F}}\right)^{\mathbb{Z}}\right)$. The group morphism

$$
\operatorname{Red}^{\left(h_{1}, h_{2}\right)}: \operatorname{Groth}\left(\mathbb{Q}_{p}^{\times} \times G L_{n}(K) \times G L_{n}(K)\right) \rightarrow \operatorname{Groth}\left(\operatorname{Frob}_{\mathbb{F}}^{\mathbb{Z}}\right)
$$

is the composite of normalized Jacquet functors

$$
J_{i}: \operatorname{Groth}\left(G L_{n}(K)\right) \rightarrow \operatorname{Groth}\left(G L_{n-h_{i}}(K) \times G L_{h_{i}}(K)\right)
$$


for $i=1,2$ with the map

$\operatorname{Groth}\left(\mathbb{Q}_{p}^{\times} \times G L_{n-h_{1}}(K) \times G L_{h_{1}}(K) \times G L_{n-h_{2}}(K) \times G L_{h_{2}}(K)\right) \rightarrow \operatorname{Groth}\left(\operatorname{Frob}_{\mathbb{F}}^{\mathbb{Z}}\right)$

which sends $\left[\alpha_{1} \otimes \beta_{1} \otimes \alpha_{2} \otimes \beta_{2} \otimes \gamma\right]$ to

$$
\begin{aligned}
& \sum_{\phi_{1}, \phi_{2}} \operatorname{vol}\left(D_{K, n-h_{1}}^{\times} / K^{\times}\right)^{-1} \cdot \operatorname{vol}\left(D_{K, n-h_{2}}^{\times} / K^{\times}\right) \cdot \operatorname{tr} \alpha_{1}\left(\varphi_{\operatorname{Sp}_{n-h_{1}}\left(\phi_{1}\right)}\right) \cdot \operatorname{tr} \alpha_{2}\left(\varphi_{\operatorname{Sp}_{n-h_{2}}\left(\phi_{2}\right)}\right) \cdot
\end{aligned}
$$

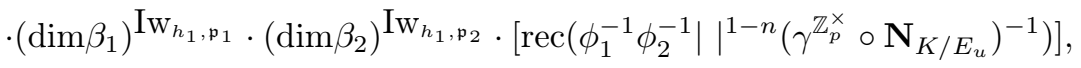

where the sum is over characters $\phi_{1}, \phi_{2}$ of $K^{\times} / \mathcal{O}_{K}^{\times}$.

Lemma 5.3.2. We have the following equality in $\operatorname{Groth}\left(G\left(\mathbb{A}^{\infty, p}\right) \times\left(\operatorname{Frob}_{\mathbb{F}}\right)^{\mathbb{Z}}\right)$ :

$$
\begin{gathered}
B C^{p}\left(H\left(Y_{\mathrm{Iw}(\vec{m}), S, T}, \mathcal{L}_{\xi}\right)\left[\Pi^{1, \mathfrak{S}}\right]\right)=e_{0} C_{G}\left[\iota_{l}^{-1} \Pi^{1, \infty, p}\right] \operatorname{dim}\left[\left(\otimes_{i=3}^{r} \pi_{\mathfrak{p}_{i}}\right)^{\left.U_{p}^{\mathfrak{p}_{1} \mathfrak{p}_{2}}\right]}\right] \times \\
\left(\sum_{h_{1}=0}^{n-\# S} \sum_{h_{2}=0}^{n-\# T}(-1)^{2 n-\# S-\# T-h_{1}-h_{2}}\left(\begin{array}{c}
n-\# S \\
h_{1}
\end{array}\right)\left(\begin{array}{c}
n-\# T \\
h_{2}
\end{array}\right) .\right. \\
\operatorname{Red}^{\left(h_{1}, h_{2}\right)}\left(\pi_{p, 0} \otimes \pi_{\mathfrak{p}_{1}} \otimes \pi_{\left.\mathfrak{p}_{2}\right)}\right) .
\end{gathered}
$$

Proof. The proof is a straightforward generalization of the proof of Lemma 4.3 of $[\mathrm{TY}]$.

Theorem 5.3.3. Assume that $\Pi_{\mathfrak{p}_{1}}^{0} \simeq \Pi_{\mathfrak{p}_{2}}^{0}$ has an Iwahori fixed vector. Then $\Pi_{\mathfrak{p}_{1}}^{0} \simeq \Pi_{\mathfrak{p}_{2}}^{0}$ is tempered

Proof. By Corollary VII.2.18 of [HT], $\iota_{l} \pi_{\mathfrak{p}_{i}}$ is tempered if and only if, for all $\sigma \in W_{K}$ every eigenvalue $\alpha$ of $\mathcal{L}_{n, K}\left(\Pi_{\mathfrak{p}_{i}}^{0}\right)(\sigma)$ (where $\mathcal{L}_{n, K}\left(\Pi_{\mathfrak{p}_{i}}^{0}\right)$ is the image of 
$\Pi_{\mathfrak{p}_{i}}^{0}$ under the local Langlands correspondence, normalized as in [Sh3]) satisfies

$$
\left|\iota_{l} \alpha\right|^{2} \in q^{\mathbb{Z}} .
$$

We shall first use a standard argument to show that we can always ensure that

$$
\left|\iota_{l} \alpha\right|^{2} \in q^{\frac{1}{2} \mathbb{Z}}
$$

and then we will use a classification of irreducible, generic, $\iota$-preunitary representations of $G L_{n}(K)$ together with the cohomology of Igusa varieties to show the full result.

The space $H^{k}\left(X, \mathcal{L}_{\xi}\right)$ decomposes as a $G\left(\mathbb{A}^{\infty}\right)$-module as

$$
H^{k}\left(X, \mathcal{L}_{\xi}\right)=\bigoplus_{\pi^{\infty}} \pi^{\infty} \otimes R_{\xi, l}^{k}\left(\pi^{\infty}\right)
$$

where $\pi^{\infty}$ runs over $\operatorname{Irr}_{l}\left(G\left(\mathbb{A}^{\infty}\right)\right)$ and $R_{\xi . l}^{k}\left(\pi^{\infty}\right)$ is a finite-dimensional $\operatorname{Gal}(\bar{F} / F)$ representation. Define the $\operatorname{Gal}(\bar{F} / F)$-representation

$$
\tilde{R}_{l}^{k}\left(\Pi^{1}\right)=\sum_{\pi_{\infty}} R_{\xi, l}^{k}\left(\pi^{\infty}\right)
$$

where the sum is over the $\pi^{\infty} \in \operatorname{Irr}_{l}\left(G\left(\mathbb{A}^{\infty}\right)\right)$ which are cohomological, unramified outside $\mathfrak{S}_{\text {fin }}$ and such that $B C\left(\iota_{l} \pi^{\infty}\right)=\Pi^{1, \infty}$. Also define the element $\tilde{R}_{l}\left(\Pi^{1}\right) \in \operatorname{Groth}(\operatorname{Gal}(\bar{F} / F))$ by

$$
\tilde{R}_{l}\left(\Pi^{1}\right)=\sum_{k}(-1)^{k} \tilde{R}_{l}^{k}\left(\Pi^{1}\right)
$$

We claim that we have the following identity in $\operatorname{Groth}\left(W_{K}\right)$ :

$$
\tilde{R}_{l}\left(\Pi^{1}\right)=e_{0} C_{G} \cdot\left[\left.\left(\pi_{p, 0} \circ \operatorname{Art}_{\mathbb{Q}_{p}}^{-1}\right)\right|_{W_{K}} \otimes \iota_{l}^{-1} \mathcal{L}_{n, K}\left(\Pi_{\mathfrak{p}_{1}}^{0}\right) \otimes \iota_{l}^{-1} \mathcal{L}_{n, K}\left(\Pi_{\mathfrak{p}_{2}}^{0}\right)\right]
$$


This can be deduced from results of Kottwitz [Ko2] or by combining Theorem 5.3.1 with Mantovan's formula [Man].

From the above identity, using the fact that $\Pi_{\mathfrak{p}_{1}}^{0} \simeq \Pi_{\mathfrak{p}_{2}}^{0}$, we see that $\left|\iota_{l}(\alpha \beta)\right|^{2} \in$ $q^{\mathbb{Z}}$ for any eigenvalues $\alpha, \beta$ of any $\sigma \in W_{K}$, since $\tilde{R}_{l}\left(\Pi^{1}\right)$ is found in the cohomology of some proper, smooth variety $X_{U}$ over $K$. In particular, we know that $\left|\iota_{l} \alpha\right|^{2} \in q^{\frac{1}{2} \mathbb{Z}}$. Moreover, if one eigenvalue $\alpha$ of $\sigma$ satisfies $\left|\iota_{l} \alpha\right|^{2} \in q^{\mathbb{Z}}$ then all other eigenvalues of $\sigma$ would be forced to satisfy it as well. A result of Tadic ([Tad], see also Lemma I.3.8 of [HT]) says that if $\pi_{\mathfrak{p}_{i}}$ is a generic, $\iota_{l}$-preunitary representation of $G L_{n}(K)$ with central character $\left|\psi_{\pi_{\mathfrak{p}_{i}}}\right| \equiv 1$ then $\pi_{\mathfrak{p}_{i}}$ is isomorphic to

$\mathrm{n}-\operatorname{Ind}_{P(K)}^{G L_{n}(K)}\left(\pi_{1} \times \cdots \times \pi_{s} \times \pi_{1}^{\prime}|\operatorname{det}|^{a_{1}} \times \pi_{1}^{\prime}|\operatorname{det}|^{-a_{1}} \times \cdots \times \pi_{t}^{\prime}|\operatorname{det}|^{a_{t}} \times \pi_{t}^{\prime}|\operatorname{det}|^{-a_{t}}\right)$,

for some parabolic subgroup $P$ of $G L_{n}$. The $\pi_{1}, \ldots, \pi_{s}, \pi_{1}^{\prime}, \ldots \pi_{t}^{\prime}$ are square integrable representations of smaller linear groups with $\left|\psi_{\pi_{j}}\right| \equiv\left|\psi_{\pi_{j^{\prime}}^{\prime}}\right| \equiv 1$ for all $j, j^{\prime}$. Moreover, we must have $0<a_{j}<\frac{1}{2}$ for $j=1, \ldots, t$. If $s \neq 0$ then for any $\sigma \in W_{K}$ there is an eigenvalue $\alpha$ of $\mathcal{L}_{K, n}\left(\pi_{\mathfrak{p}_{i}}\right)(\sigma)$ with $\left|\iota_{l} \alpha\right|^{2} \in q^{\mathbb{Z}}$, but then this must happen for all eigenvalues of $\mathcal{L}_{K, n}\left(\pi_{\mathfrak{p}_{i}}\right)(\sigma)$. So then $t=0$ and $\pi_{\mathfrak{p}_{i}}$ is tempered. If $s=0$ then every eigenvalue $\alpha$ of a lift of Frobenius $\sigma \in W_{K}$ must satisfy

$$
\left|\iota_{l} \alpha\right|^{2} \in q^{\mathbb{Z} \pm 2 a_{j}}
$$

for some $j \in 1, \ldots, t$. Note that each $j$ corresponds to at least one such eigenvalue $\alpha$, so we must have $a_{j}=\frac{1}{4}$ for all $j=1, \ldots, t$. To summarize, $\pi_{\mathfrak{p}_{i}}$ is either tempered or it is of the form

$$
\mathrm{n}-\operatorname{Ind}_{P(K)}^{G L_{n}(K)}\left(\pi_{1}^{\prime}|\operatorname{det}|^{\frac{1}{4}} \times \pi_{1}^{\prime}|\operatorname{det}|^{-\frac{1}{4}} \times \cdots \times \pi_{t}^{\prime}|\operatorname{det}|^{\frac{1}{4}} \times \pi_{t}^{\prime}|\operatorname{det}|^{-\frac{1}{4}}\right) .
$$

We shall now focus on the second case, in order to get a contradiction. Since 
$\pi_{\mathfrak{p}_{i}}$ has an Iwahori fixed vector, each $\pi_{j}^{\prime}$ must be equal to $\operatorname{Sp}_{s_{j}}\left(\chi_{j}\right)$, where $\chi_{j}$ is an unramified character of $K^{\times}$. We can compute $\operatorname{Red}^{\left(h_{1}, h_{2}\right)}\left(\pi_{p, 0} \otimes \pi_{\mathfrak{p}_{1}} \otimes \pi_{\mathfrak{p}_{2}}\right)$ explicitly and compare it to the cohomology of a closed stratum $Y_{\mathrm{Iw}, S, T}$ via Lemma 5.3.2.

We can compute $\operatorname{Red}^{\left(h_{1}, h_{2}\right)}\left(\pi_{p, 0} \otimes \pi_{\mathfrak{p}_{1}} \otimes \pi_{\mathfrak{p}_{2}}\right)$ using an analogue of Lemma I.3.9 of [HT], which follows as well from Lemma 2.12 of [BZ]. Indeed,

$J_{i}\left(\mathrm{n}-\operatorname{Ind}_{P(K)}^{G L_{n}(K)}\left(\operatorname{Sp}_{s_{1}}\left(\chi_{1}\right) \cdot|\operatorname{det}|^{\frac{1}{4}} \times \operatorname{Sp}_{s_{1}}\left(\chi_{1}\right) \cdot|\operatorname{det}|^{-\frac{1}{4}} \times \cdots \times \operatorname{Sp}_{s_{t}}\left(\chi_{t}\right) \cdot|\operatorname{det}|^{-\frac{1}{4}}\right)\right)$

is equal to

$$
\begin{aligned}
& \sum\left[\mathrm{n}-\operatorname{Ind}_{P_{i}^{\prime}(K)}^{G L_{h_{i}(k)}}\left(\left(\operatorname{Sp}_{l_{1}}\left(\chi_{1} \otimes|\operatorname{det}|^{s_{1}-l_{1}+\frac{1}{4}}\right) \times \cdots \times \operatorname{Sp}_{k_{t}}\left(\chi_{t} \otimes|\operatorname{det}|^{s_{t}-k_{t}-\frac{1}{4}}\right)\right)\right]\right. \\
& {\left[\mathrm{n}-\operatorname{Ind}_{P_{i}^{\prime \prime}(K)}^{G L_{h_{i}(k)}}\left(\left(\operatorname{Sp}_{s_{1}-l_{1}}\left(\chi_{1} \otimes|\operatorname{det}|^{\frac{1}{4}}\right) \times \cdots \times \operatorname{Sp}_{s_{t}-k_{t}}\left(\chi_{t} \otimes|\operatorname{det}|^{-\frac{1}{4}}\right)\right)\right],\right.}
\end{aligned}
$$

where the sum is over all non-negative integers $l_{j}, k_{j} \leq s_{j}$ with $h_{i}=\sum_{j=1}^{t}\left(l_{j}+\right.$ $\left.k_{j}\right)$. Here $P_{i}^{\prime}$ and $P_{i}^{\prime \prime}$ are parabolic sungroups with Levi components $G L_{l_{1}} \times$ $\cdots \times G L_{k_{t}}$ and $G L_{s_{1}-l_{1}} \times \cdots \times G L_{s_{t}-k_{t}}$ respectively.

Let $V_{j_{1} j_{2}}^{k}=\operatorname{rec}\left(\chi_{j_{1}}^{-1} \chi_{j_{2}}^{-1}||^{1-n+\epsilon_{k}}\left(\psi_{u} \circ \mathbf{N}_{K / E_{u}}\right)^{-1}\right)$, where

$$
\epsilon_{k}= \begin{cases}-\frac{1}{2} \text { if } & k=1 \\ 0 \text { if } & k=2 \\ \frac{1}{2} \text { if } & k=3\end{cases}
$$

After we apply the functor

$\operatorname{Groth}\left(G L_{n-h_{1}}(K) \times G L_{h_{1}}(K) \times G L_{n-h_{2}}(K) \times G L_{h_{2}}(K) \times \mathbb{Q}_{p}^{\times}\right) \rightarrow \operatorname{Groth}\left(\operatorname{Frob}_{\mathbb{F}}^{\mathbb{Z}}\right)$, 
we get

$$
\operatorname{Red}^{\left(h_{1}, h_{2}\right)}\left(\pi_{p, 0} \otimes \pi_{\mathfrak{p}_{1}} \otimes \pi_{\mathfrak{p}_{2}}\right)=\sum_{j_{1}, j_{2}, k} \gamma_{j_{1} j_{2}}^{\left(h_{1}, h_{2}\right)}\left(\left[V_{j_{1} j_{2}}^{1}\right] \oplus 2\left[V_{j_{1} j_{2}}^{2}\right] \oplus\left[V_{j_{1} j_{2}}^{3}\right]\right),
$$

where

$$
\begin{aligned}
& \gamma_{j_{1}, j_{2}}^{\left(h_{1}, h_{2}\right)}=\prod_{i=1}^{2} \operatorname{dim}\left(\mathrm{n}-\operatorname{Ind}_{P_{i}^{\prime}(K)}^{G L_{h_{i}}(K)}\left(\operatorname{Sp}_{s_{j_{i}}+h_{i}-n}\left(\chi_{j_{i}}||^{n-h_{i} \pm \frac{1}{4}}\right) \otimes \operatorname{Sp}_{s_{j}}\left(\chi_{j_{i}}||^{\mp \frac{1}{4}}\right)\right.\right. \\
& \left.\left.\otimes \bigotimes_{j \neq j_{i}} \operatorname{Sp}_{s_{j}}\left(\chi_{j}||^{\frac{1}{4}}\right) \otimes \bigotimes_{j \neq j_{i}} \operatorname{Sp}_{s_{j}}\left(\chi_{j}||^{-\frac{1}{4}}\right)\right)\right)^{\mathrm{Iw}_{h_{i}, \mathrm{p}_{i}}} \\
& =\prod_{i=1}^{2} \frac{h_{i} !}{\left(s_{j_{i}}+h_{i}-n\right) ! s_{j_{i}} ! \prod_{j \neq j_{i}}\left(s_{j} !\right)^{2}}
\end{aligned}
$$

and where the sum is over the $j_{1}, j_{2}$ for which $s_{j_{i}} \geq n-h_{i}$ for $i=1,2$. Here $P_{i}^{\prime}$ for $i=1,2$ are parabolic subgroups of $G L_{h_{i}}(K)$.

Let $D\left(\Pi^{1}\right)=e_{0} C_{G}\left[\Pi^{1, \infty, p}\right] \operatorname{dim}\left[\left(\otimes_{i=3}^{r} \pi_{\mathfrak{p}_{i}}\right)^{U_{p}^{\mathfrak{p}_{1} \mathfrak{p}_{2}}}\right]$. Then

$$
\begin{gathered}
B C^{p}\left(H\left(Y_{\mathrm{Iw}(\vec{m}), S, T}, \mathcal{L}_{\xi}\right)\left[\Pi^{1, \mathfrak{S}}\right]\right)=D\left(\Pi^{1}\right) \\
\left(\sum_{h_{1}=0}^{n-\# S} \sum_{h_{2}=0}^{n-\# T}(-1)^{2 n-\# S-\# T-h_{1}-h_{2}}\left(\begin{array}{c}
n-\# S \\
h_{1}
\end{array}\right)\left(\begin{array}{c}
n-\# T \\
h_{2}
\end{array}\right)\right. \\
\left.\sum_{j_{1}, j_{2}, k} \gamma_{j_{1} j_{2}}^{\left(h_{1}, h_{2}\right)}\left(\left[V_{j_{1} j_{2}}^{1}\right] \oplus 2\left[V_{j_{1} j_{2}}^{2}\right] \oplus\left[V_{j_{1} j_{2}}^{3}\right]\right)\right)
\end{gathered}
$$

We can compute the coefficient of $\left[V_{j_{1} j_{2}}^{k}\right]$ in $B C^{p}\left(H\left(Y_{\mathrm{Iw}, S, T}, \mathcal{L}_{\xi}\right)\right)\left[\Pi^{1, \mathfrak{S}}\right]$ by summing first over $j_{1}, j_{2}$ and then over $h_{1}, h_{2}$ going from $n-s_{j_{1}}, n-s_{j_{2}}$ to $n-\# S$ and $n-\# T$ respectively. Note that the coefficient of $\left[V_{j_{1} j_{2}}^{2}\right]$ is exactly twice that 
of $\left[V_{j_{1} j_{2}}^{1}\right]$ and of $\left[V_{j_{1} j_{2}}^{3}\right]$. The sum we get for $\left[V_{j_{1} j_{2}}^{1}\right]$ is

$$
\begin{gathered}
D\left(\Pi^{1}\right) \frac{(n-\# S) !(n-\# T) !}{\left(s_{j_{1}}-\# S\right) !\left(s_{j_{2}}-\# T\right) ! s_{j_{1}} ! s_{j_{2}} ! \prod_{j \neq j_{1}}\left(s_{j} !\right)^{2} \prod_{j \neq j_{2}}\left(s_{j} !\right)^{2}} \\
\left(\sum_{h_{1}=n-s_{j_{1}}}^{n-\# S} \sum_{h_{2}=n-s_{j_{2}}}^{n-\# T}(-1)^{2 n-\# S-\# T-h_{1}-h_{2}}\left(\begin{array}{c}
s_{j_{1}}-\# S \\
h_{1}+s_{j_{1}}-n
\end{array}\right)\left(\begin{array}{c}
s_{j_{2}}-\# T \\
h_{2}+s_{j_{2}-n}
\end{array}\right)\right)
\end{gathered}
$$

The sum in parentheses can be decomposed as

$$
\begin{gathered}
\left(\sum_{h_{1}=n-s_{j_{1}}}^{n-\# S}(-1)^{n-\# S-h_{1}}\left(\begin{array}{c}
s_{j_{1}}-\# S \\
h_{1}+s_{j_{1}}-n
\end{array}\right)\right) . \\
\left(\sum_{h_{2}=n-s_{j_{2}}}^{n-\# T}(-1)^{n-\# T-h_{2}}\left(\begin{array}{c}
s_{j_{2}}-\# T \\
h_{2}+s_{j_{2}}-n
\end{array}\right)\right),
\end{gathered}
$$

which is equal to 0 unless both $s_{j_{1}}=\# S$ and $s_{j_{2}}=\# T$. So

$$
\begin{gathered}
B C^{p}\left(H\left(Y_{\operatorname{Iw}(\vec{m}), S, T}, \mathcal{L}_{\xi}\right)\left[\Pi^{1, \mathfrak{S}}\right]\right)=D\left(\Pi^{1}\right) \cdot \sum_{s_{j_{1}}=\# S, s_{j_{2}}=\# T} \frac{(n-\# S) !(n-\# T) ! s_{j_{1}} ! s_{j_{2}} !}{\prod_{j}\left(s_{j} !\right)^{4}} . \\
\left(\left[V_{j_{1} j_{2}}^{1}\right]+2\left[V_{j_{1} j_{2}}^{2}\right]+\left[V_{j_{1} j_{2}}^{3}\right]\right) .
\end{gathered}
$$

Since each $Y_{U, S, T}$ is proper and smooth, it follows from the Weil conjectures that $H^{j}\left(Y_{\operatorname{Iw}(\vec{m}), S, T}, \mathcal{L}_{\xi}\right)$ is strictly pure of weight $m_{\xi}-2 t_{\xi}+j$. This

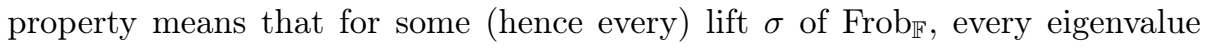

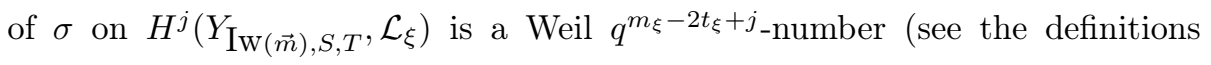
above Lemma 1.4 of $[\mathrm{TY}])$. However, the $\left[V_{j_{1} j_{2}}^{k}\right]$ are strictly pure of weight $m_{\xi}-2 t_{\xi}+2 n-2-\epsilon_{k}-(\# S-1)-(\# T-1)=m_{\xi}-2 t_{\xi}+2 n-\# S-\# T-2 \epsilon_{k}$. So

$$
B C^{p}\left(H^{j}\left(Y_{\operatorname{Iw}(\vec{m}), S, T}, \mathcal{L}_{\xi}\right)\left[\Pi^{1, \mathfrak{S}}\right]\right)=0
$$


unless $j=2 n-\# S-\# T \pm 1$ or $j=2 n-\# S-\# T$. However, if the Igusa cohomology is non-zero for some $j=2 n-\# S-\# T \pm 1$, then there exist $j_{1}, j_{2}$ with $s_{j_{1}}=\# S$ and $s_{j_{2}}=\# T$. Hence, the cohomology must also be non-zero for $j=2 n-\# S-\# T$. The coefficients of $\left[V_{j_{1} j_{2}}^{k}\right]$ all have the same sign, so they are either strictly positive or strictly negative only depending on $D\left(\Pi^{1}\right)$. However, $B C^{p}\left(H\left(Y_{\operatorname{Iw}(m), S, T}, \mathcal{L}_{\xi}\right)\left[\Pi^{1, \mathfrak{S}}\right]\right.$ is an alternating sum, so the weight $2 n-\# S-\# T \pm 1$ part of the cohomology should appear with a different sign from the weight $2 n-\# S-\# T$ part. This is a contradiction, so it must be the case that $\pi_{\mathfrak{p}_{1}} \simeq \pi_{\mathfrak{p}_{2}}$ is tempered.

Corollary 5.3.4. Let $n \in \mathbb{Z}_{\geq 2}$ be an integer and $L$ be any $C M$ field. Let $\Pi$ be a cuspidal automorphic representation of $G L_{n}\left(\mathbb{A}_{L}\right)$ satisfying

$$
\text { - } \Pi^{\vee} \simeq \Pi \circ c
$$

- $\Pi_{\infty}$ is cohomological for some irreducible algebraic representation $\Xi$.

Then $\Pi$ is tempered at every finite place $w$ of $L$.

Proof. By Lemma 1.4.3 of [TY], an irreducible smooth representation $\Pi$ of $G L_{n}(K)$ is tempered if and only if $\mathcal{L}_{K, n}(\Pi)$ is pure of some weight. By Lemma 1.4.1 of [TY], purity is preserved under a restriction to the Weil-Deligne representation of $W_{K^{\prime}}$ for a finite extension $K^{\prime} / K$ of fields.

Fix a place $v$ of $L$ above $p$ where $p \neq l$. We will find a CM field $F^{\prime}$ such that

- $F^{\prime}=E F_{1}$, where $E$ is an imaginary quadratic field in which $p$ splits and $F_{1}=\left(F^{\prime}\right)^{c=1}$ has $\left[F_{1}: \mathbb{Q}\right] \geq 2$,

- $F^{\prime}$ is soluble and Galois over $L$,

- $\Pi_{F^{\prime}}^{0}=B C_{F^{\prime} / L}(\Pi)$ is a cuspidal automorphic representation of $G L_{n}\left(\mathbb{A}_{F^{\prime}}\right)$, and

- there is a place $\mathfrak{p}$ of $F$ above $v$ such that $\Pi_{F^{\prime}, \mathfrak{p}}^{0}$ has an Iwahori fixed vector, 
and a $\mathrm{CM}$ field $F$ which is a quadratic extension of $F^{\prime}$ such that

- $\mathfrak{p}=\mathfrak{p}_{1} \mathfrak{p}_{2}$ splits in $F$,

- $\operatorname{Ram}_{F / \mathbb{Q}} \cup \operatorname{Ram}_{\mathbb{Q}}(\varpi) \cup \operatorname{Ram}_{\mathbb{Q}}(\Pi) \subset \operatorname{Spl}_{F / F^{\prime}, \mathbb{Q}}$, and

- $\Pi_{F}^{0}=B C_{F / F^{\prime}}\left(\Pi_{F^{\prime}}^{0}\right)$ is a cuspidal automorphic representation of $G L_{n}\left(\mathbb{A}_{F}\right)$.

To find $F^{\prime}$ and $F$ we proceed as follows, using the same argument as on the last page of [Sh3]. For a CM field $F$, we shall use the sets $\mathcal{E}(F)$ and $\mathcal{F}(F)$, which are defined in the proof of Theorem 7.5 of [Sh3].

First we find a CM field $F_{0}$ which is soluble and Galois over $L$ and a place $\mathfrak{p}_{0}$ above $v$ such that the last two conditions for $F^{\prime}, \mathfrak{p}$ are satisfied for $F_{0}, \mathfrak{p}_{0}$ instead. To see that the second to last condition for $F^{\prime}$ only eliminates finitely many choices for the $\mathrm{CM}$ field we can use the same argument as Clozel in Section 1 of [Cl2]. Indeed, if $B C_{F^{\prime} / L}(\Pi)$ is not cuspidal, then we would have $\Pi \otimes \epsilon \simeq \Pi$ for $\epsilon$ the Artin character of $L$ associated to $F^{\prime}$. But then the character $\epsilon$ would occur in the semisimplification of $R_{l} \otimes R_{l} \otimes \omega^{n-1}$, where $R_{l}$ is the Galois representation associated to $\Pi$ by Chenevier and Harris in $[\mathrm{CH}]$ and $\omega$ is the cyclotomic character. Thus, there are only finitely many choices for $\epsilon$ and so for $F^{\prime} / L$ which are excluded.

Next, we choose $E \in \mathcal{E}\left(F_{0}\right)$ such that $p$ splits in $E$. We take $F^{\prime}=E F_{0}$ and $\mathfrak{p}$ any place of $F^{\prime}$ above $\mathfrak{p}_{0}$. Let $F_{1}$ be the maximal totally real subfield of $F^{\prime}$ and let $w$ be the place of $F_{1}$ below $\mathfrak{p}$. Next, we pick $F^{\prime \prime} \in \mathcal{F}\left(F^{\prime}\right)$ different from $F^{\prime}$ and such that $w$ splits in $F^{\prime \prime}$. Take $F=F^{\prime \prime} F^{\prime}$.

We can find a character $\psi$ of $\mathbb{A}_{E}^{\times} / E^{\times}$such that $\Pi^{1}=\psi \otimes \Pi_{F}^{0}$ together with $F$ satisfy the assumptions in the beginning of the section. (For the specific conditions that $\psi$ must satisfy, see Lemma 7.0.11.) We also know that $\Pi_{F, \mathfrak{p}_{1}}^{0} \simeq$ $\Pi_{F, \mathfrak{p}_{2}}^{0}$ has an Iwahori fixed vector, thus we are in the situation of Theorem 5.3.3. 
Proposition 5.3.5. Assume again that the conditions in the beginning of this section are satisfied and that $\Pi_{\mathfrak{p}_{1}}^{0} \simeq \Pi_{\mathfrak{p}_{2}}^{0}$ has a nonzero Iwahori fixed vector. Then

$$
B C^{p}\left(H^{j}\left(Y_{\mathrm{Iw}(\vec{m}), S, T}, \mathcal{L}_{\xi}\right)\left[\Pi^{1, \mathfrak{S}}\right]\right)=0
$$

unless $j=2 n-\# S-\# T$.

Proof. We will go through the same computation as in the proof of Theorem 5.3.3 except we will use the fact that $\pi_{\mathfrak{p}_{1}} \simeq \pi_{\mathfrak{p}_{2}}$ is tempered, so it is of the form

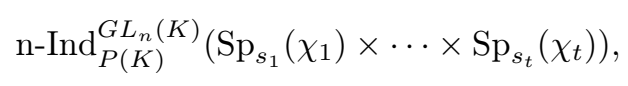

where the $\chi_{j}$ are unramified characters of $K^{\times}$.

We can compute $\operatorname{Red}^{\left(h_{1}, h_{2}\right)}\left(\pi_{p, 0} \otimes \pi_{\mathfrak{p}_{1}} \otimes \pi_{\mathfrak{p}_{2}}\right)$ as in the proof of Theorem 5.3 .3 .

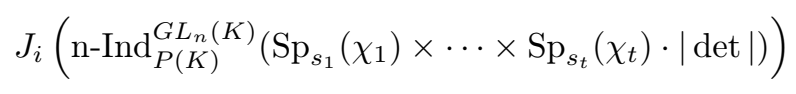

is equal to

$$
\begin{aligned}
& \sum\left[\mathrm{n}-\operatorname{Ind}_{P_{i}^{\prime}(K)}^{G L_{h_{i}(k)}}\left(\operatorname{Sp}_{k_{1}}\left(\chi_{1} \otimes|\operatorname{det}|^{s_{1}-k_{1}}\right) \times \cdots \times \operatorname{Sp}_{k_{t}}\left(\chi_{t} \otimes|\operatorname{det}|^{s_{t}-k_{t}}\right)\right)\right] \\
& {\left[\mathrm{n}-\operatorname{Ind}_{P_{i}^{\prime \prime}(K)}^{G L_{h_{i}(k)}}\left(\operatorname{Sp}_{s_{1}-k_{1}}\left(\chi_{1}\right) \times \cdots \times \operatorname{Sp}_{s_{t}-k_{t}}\left(\chi_{t}\right)\right)\right]}
\end{aligned}
$$

where the sum is over all non-negative integers $k_{j} \leq s_{j}$ with $h_{i}=\sum_{j=1}^{t} k_{j}$.

Let $V_{j_{1} j_{2}}=\operatorname{rec}\left(\chi_{j_{1}}^{-1} \chi_{j_{2}}^{-1}||^{1-n}\left(\psi_{u} \circ \mathbf{N}_{K / E_{u}}\right)^{-1}\right)$. After we apply the functor

$\operatorname{Groth}\left(G L_{n-h_{1}}(K) \times G L_{h_{1}}(K) \times G L_{n-h_{2}}(K) \times G L_{h_{2}}(K) \times \mathbb{Q}_{p}^{\times}\right) \rightarrow \operatorname{Groth}\left(\operatorname{Frob}_{\mathbb{F}}^{\mathbb{Z}}\right)$,

we get

$$
\operatorname{Red}^{\left(h_{1}, h_{2}\right)}\left(\pi_{p, 0} \otimes \pi_{\mathfrak{p}_{1}} \otimes \pi_{\mathfrak{p}_{2}}\right)=\sum_{j_{1}, j_{2}, k} \gamma_{j_{1} j_{2}}^{\left(h_{1}, h_{2}\right)}\left[V_{j_{1} j_{2}}\right]
$$


where

$$
\begin{gathered}
\gamma_{j_{1}, j_{2}}^{\left(h_{1}, h_{2}\right)}=\prod_{i=1}^{2} \operatorname{dim}\left(\mathrm{n}-\operatorname{Ind}_{P_{i}^{\prime}(K)}^{G L_{h_{i}}(K)}\left(\operatorname{Sp}_{s_{j_{i}}}\left(\chi_{j_{i}}||^{n-h_{i}}\right) \otimes \bigotimes_{j \neq j_{i}} \operatorname{Sp}_{s_{j}}\left(\chi_{j}\right)\right)\right)^{\operatorname{Iw}_{h_{i}, \mathrm{p}_{i}}} \\
=\prod_{i=1}^{2} \frac{h_{i} !}{\left(s_{j_{i}}+h_{i}-n\right) ! s_{j_{i}} ! \prod_{j \neq j_{i}}\left(s_{j} !\right)^{2}}
\end{gathered}
$$

and where the sum is over the $j_{1}, j_{2}$ for which $s_{j_{i}} \geq n-h_{i}$ for $i=1,2$. Here $P_{i}^{\prime}$ for $i=1,2$ are parabolic subgroups of $G L_{h_{i}}(K)$.

Let $D\left(\Pi^{1}\right)=e_{0} C_{G}\left[\Pi^{1, \infty, p}\right] \operatorname{dim}\left[\left(\otimes_{i=3}^{r} \pi_{\mathfrak{p}_{i}}\right)^{U_{p}^{\mathfrak{p}_{1} \mathfrak{p}_{2}}}\right]$. The same computation as in the proof of Theorem 5.3.3 gives us

$$
\begin{gathered}
B C^{p}\left(H\left(Y_{\operatorname{Iw}(\vec{m}), S, T}, \mathcal{L}_{\xi}\right)\left[\Pi^{1, \mathfrak{S}}\right]\right) \\
=D\left(\Pi^{1}\right) \cdot \sum_{s_{j_{1}}=\# S, s_{j_{2}}=\# T} \frac{(n-\# S) !(n-\# T) ! s_{j_{1}} ! s_{j_{2}} !}{\prod_{j}\left(s_{j} !\right)^{2}}\left[V_{j_{1} j_{2}}\right] .
\end{gathered}
$$

Since $\pi_{\mathfrak{p}_{1}} \simeq \pi_{\mathfrak{p}_{2}}$ is tempered, we know that $\left[V_{j_{1} j_{2}}\right]$ is strictly pure of weight $2 n-$ $\# S-\# T$. The Weil conjectures tell us then that $B C^{p}\left(H^{j}\left(Y_{\operatorname{Iw}(\vec{m}), S, T}, \mathcal{L}_{\xi}\right)\left[\Pi^{1, \mathfrak{S}}\right]\right)=$ 0 unless $j=2 n-\# S-\# T$. 


\section{Chapter 6}

\section{The cohomology of Igusa varieties}

The goal of this chapter is to explain how to prove Theorem 5.3.1. The proof will be a straightforward generalization of the proof of Theorem 6.1 of [Sh3] and so we will follow closely the argument and the notation of that paper.

We will summarize without proof the results in [Sh3] on transfer and on the twisted trace formula. We will emphasize the place $\infty$, since that is the only place of $\mathbb{Q}$ where our group $G$ differs from the group $G$ considered in [Sh3]. All of the results and notation are as in [Sh3], except in the proof of Lemmas 6.0.8 and 6.0.9, where we also use the notation of [Sh2].

We start by explaining the notation we will be using throughout this section, which is consistent with the notation of [Sh3]. Recall that we have fixed a unitary similitude group $G$ over $\mathbb{Q}$, which satisfies certain local conditions as in Lemma 2.1.1. In this section, will work with a quasi-split form of $G$, denoted by $G_{n}$ as well as with groups $G_{n_{1}, n_{2}}$ which are endoscopic groups for $G_{n}$. We will denote an element in the set $\left.\left\{G_{n}\right\} \cup\left\{G_{n_{1}, n_{2}} \mid n_{1}+n_{2}=n, n_{1} \geq n_{2}>0\right)\right\}$ as $G_{\vec{n}}$, where 
$\vec{n}$ is a multiset of positive integers (in our case, $\vec{n}$ will have length 1 or 2 ). In other words, $\vec{n}$ runs through the elements of the set $\{n\} \cup\left\{\left(n_{1}, n_{2}\right) \mid n_{1}+n_{2}=\right.$ $\left.n, n_{1} \geq n_{2}>0\right\}$.

If $r \in\{1,2\}$ and $\vec{n}=\left(n_{i}\right)_{i=1}^{r}$ with $n_{i} \in \mathbb{Z}_{>0}$ define

$$
G L_{\vec{n}}:=\prod_{i=1}^{r} G L_{n_{i}} .
$$

Let $i_{\vec{n}}: G L_{\vec{n}} \hookrightarrow G L_{N}\left(N=\sum_{i} n_{i}\right)$ be the natural map. Let

$$
\Phi_{\vec{n}}=i_{\vec{n}}\left(\Phi_{n_{1}}, \ldots,, \Phi_{n_{j}}\right)
$$

where $\Phi_{n}$ is the matrix in $G L_{n}$ with entries $\left(\Phi_{n}\right)_{i j}=(-1)^{i+1} \delta_{i, n+1-j}$.

Let $K$ be some local non-archimedean local field and $H$ a connected reductive group over $K$. We will denote by $\operatorname{Irr}(H(K))$ (resp. $\left.\operatorname{Irr}_{l}(H(K))\right)$ the set of isomorphism classes of irreducible admissible representations of $G(K)$ over $\mathbb{C}$ (resp. over $\overline{\mathbb{Q}}_{l}$ ). Let $C_{c}^{\infty}(H(K))$ be the space of smooth compactly supported $\mathbb{C}$-valued functions on $H(K)$. Let $P$ be a $K$-rational parabolic subgroup of $H$ with a Levi subgroup $M$. For $\pi_{M} \in \operatorname{Irr}(M(K))$ and $\pi \in \operatorname{Irr}(H(K))$ we can define the normalized Jacquet module $J_{P}^{H}(\pi)$ and the normalized parabolic induction $\mathrm{n}-\operatorname{Ind}_{P}^{H} \pi_{M}$. We can define a character $\delta_{P}: M(K) \rightarrow \mathbb{R}_{>0}^{\times}$by

$$
\delta_{P}(m)=\left.|\operatorname{det}(\operatorname{ad}(m))|_{\operatorname{Lie}(P) / \operatorname{Lie}(M)}\right|_{K} .
$$

We can view $\delta_{P}$ as a character valued in $\overline{\mathbb{Q}}_{l}^{\times}$via $\iota_{l}^{-1}$.

If

$$
J^{(h)}\left(\mathbb{Q}_{p}\right) \simeq D_{K, \frac{1}{n-h}}^{\times} \times G L_{h}(K),
$$

where $K / \mathbb{Q}_{p}$ is finite, then we define $\bar{\delta}_{P\left(J^{(h)}\right)}^{\frac{1}{2}}(g):=\delta_{P_{n-h, h}}^{\frac{1}{2}}\left(g^{*}\right)$, where $g^{*} \in$ $G L_{n-h}(K) \times G L_{h}(K)$ is any element whose conjugacy class matches that of $g$. 
If

$$
J^{\left(h_{1}, h_{2}\right)} \simeq G L_{1} \times \prod_{i=1}^{2}\left(D_{F_{\mathfrak{p}_{i}}, \frac{1}{n-h_{i}}}^{\times} \times R_{F_{\mathfrak{p}_{i}} / \mathbb{Q}_{p}} G L_{h_{i}}\right) \times \prod_{i>2} R_{F_{\mathfrak{p}_{i}} / \mathbb{Q}_{p}} G L_{n}
$$

we define $\bar{\delta}_{P\left(J^{\left(h_{1}, h_{2}\right)}\right.}^{\frac{1}{2}}: J^{\left(h_{1}, h_{2}\right)}\left(\mathbb{Q}_{p}\right) \rightarrow \overline{\mathbb{Q}}_{l}^{\times}$to be the product of the characters $\bar{\delta}_{P\left(J^{\left(h_{i}\right)}\right)}^{\frac{1}{2}}$ for $i=1,2$.

Let $\vec{n}=\left(n_{i}\right)_{i=1}^{r}$ for some $r \in\{1,2\}$ and $n_{i} \in \mathbb{Z}_{>0}$. Let $G_{\vec{n}}$ be the $\mathbb{Q}$-group defined by

$$
G_{\vec{n}}(R)=\left\{\left(\lambda, g_{i}\right) \in G L_{1}(R) \times G L_{\vec{n}}\left(F \otimes_{\mathbb{Q}} R\right) \mid g_{i} \cdot \Phi_{\vec{n}} \cdot{ }^{t} g_{i}^{c}=\lambda \Phi_{\vec{n}}\right\}
$$

for any $\mathbb{Q}$-algebra $R$. For any $\vec{n}$, the group $G_{\vec{n}}$ is quasi-split over $\mathbb{Q}$. In particular, our unitary group $G$ is an inner form of $G_{n}$. Since $G$ is quasi-split at all finite places, there exists an isomorphism

$$
G \times_{\mathbb{Q}} \mathbb{A}^{\infty} \simeq G_{n} \times_{\mathbb{Q}} \mathbb{A}^{\infty}
$$

we fix such an isomorphism.

Also define

$$
\mathbb{G}_{\vec{n}}=R_{E / \mathbb{Q}}\left(G_{\vec{n}} \times \mathbb{Q} E\right) .
$$

Let $\theta$ denote the action on $\mathbb{G}_{\vec{n}}$ induced by (id, $c$ ) on $G_{\vec{n}} \times_{\mathbb{Q}} E$. Let $\epsilon: \mathbb{Z} \rightarrow\{0,1\}$ be the unique map such that $\epsilon(n) \equiv n(\bmod 2)$. Let $\varpi: \mathbb{A}_{E}^{\times} / E^{\times} \rightarrow \mathbb{C}^{\times}$be any Hecke character such that $\left.\varpi\right|_{\mathbb{A}^{\times} / \mathbb{Q}^{\times}}$is the composite of Art $\mathbb{Q}_{\mathbb{Q}}$ and the natural surjective character $W_{\mathbb{Q}} \rightarrow \operatorname{Gal}(E / \mathbb{Q}) \stackrel{\sim}{\rightarrow}\{ \pm 1\}$. Using the Artin map $\operatorname{Art}_{E}$, we view $\varpi$ as a character $W_{E} \rightarrow \mathbb{C}^{\times}$as well.

Assume that $\operatorname{Ram}_{F / \mathbb{Q}} \cup \operatorname{Ram}_{\mathbb{Q}}(\varpi) \subset \operatorname{Spl}_{F / F_{2}, \mathbb{Q}}$.

Let $\mathcal{E}^{\text {ell }}\left(G_{n}\right)$ be a set of representatives of isomorphism classes of elliptic endoscopic triples for $G_{n}$ over $\mathbb{Q}$. Then $\mathcal{E}^{\text {ell }}\left(G_{n}\right)$ can be identified with the set 
of triples

$$
\left\{\left(G_{n}, s_{n}, \eta_{n}\right\} \cup\left\{G_{n_{1}, n_{2}}, s_{n_{1}, n_{2}}, \eta_{n_{1}, n_{2}} \mid n_{1}+n_{2}=0, n_{1} \geq n_{2}>0\right\},\right.
$$

where $\left(n_{1}, n_{2}\right)$ may be excluded in some cases. As we are only interested in the stable part of the cohomology of Igusa varieties, we will not be concerned with these exclusions so we will ignore them in this paper. Here $s_{n}=1 \in \hat{G}_{n}, s_{n_{1}, n_{2}}=$ $\left(1,\left(I_{n_{1}},-I_{n_{2}}\right)\right) \in \hat{G}_{n_{1}, n_{2}}, \eta_{n}: \hat{G}_{n} \rightarrow \hat{G}_{n}$ is the identity map whereas

$$
\eta_{n_{1}, n_{2}}:\left(\lambda,\left(g_{1}, g_{2}\right)\right) \mapsto\left(\lambda,\left(\begin{array}{cc}
g_{1} & 0 \\
0 & g_{2}
\end{array}\right)\right) .
$$

We can extend $\eta_{n_{1}, n_{2}}$ to a morphism of $L$-groups, which sends $z \in W_{E}$ to

$$
\left(\varpi(z)^{-N\left(n_{1}, n_{2}\right)},\left(\begin{array}{cc}
\varpi(z)^{\epsilon\left(n-n_{1}\right)} \cdot I_{n_{1}} & 0 \\
0 & \varpi(z)^{\epsilon\left(n-n_{2}\right)} \cdot I_{n_{2}}
\end{array}\right)\right) \rtimes z .
$$

Similarly we can also define a morphism of $L$-groups

$$
\tilde{\zeta}_{n_{1}, n_{2}}:{ }^{L} \mathbb{G}_{n_{1}, n_{2}} \rightarrow^{L} \mathbb{G}_{n},
$$

which extends the map

$$
\begin{gathered}
\zeta_{n_{1}, n_{2}}: \hat{\mathbb{G}}_{n_{1}, n_{2}} \rightarrow \hat{\mathbb{G}}_{n} \\
\left(\lambda_{+}, \lambda_{-},\left(g_{\sigma, 1}, g_{\sigma, 2}\right)\right) \mapsto\left(\lambda_{+}, \lambda_{-}\left(\begin{array}{cc}
g_{\sigma, 1} & 0 \\
0 & g_{\sigma, 2}
\end{array}\right)\right)
\end{gathered}
$$

(See section 3.2 of [Sh3] for the precise definition.) We have the following com- 
mutative diagram of $L$-morphisms.

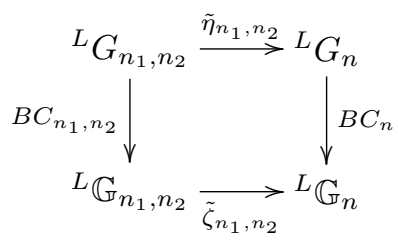

We will proceed to define local transfers for each of the arrows in the above commutative diagram so that these transfers are compatible.

Choose the normalization of the local transfer factor $\Delta_{v}(,)_{G_{\vec{n}}}^{G_{n}}$ defined in Section 3.4 of [Sh3]. It is possible to give a concrete description of the $\Delta_{v}(,)_{G_{\vec{n}}}^{G_{n}}$ transfer at finite places $v$ of $\mathbb{Q}$ between functions in $C_{c}^{\infty}\left(G_{n}\left(\mathbb{Q}_{v}\right)\right)$ and functions in $C_{c}^{\infty}\left(G_{n_{1}, n_{2}}\left(\mathbb{Q}_{v}\right)\right)$ as long as $v$ satisfies at least one of the conditions:

- $v \in \operatorname{Unr}_{F / \mathbb{Q}}$ and $v \notin \operatorname{Ram}_{\mathbb{Q}}(\varpi)$,

- $v \in \mathrm{Spl}_{E / \mathbb{Q}}$,

- $v \in \operatorname{Spl}_{F / F_{2}, \mathbb{Q}}$ and $v \notin \operatorname{Spl}_{E / \mathbb{Q}}$.

The transfer $\phi_{v}^{n_{1}, n_{2}}$ of $\phi_{v}^{n} \in C_{c}^{\infty}\left(G_{n}\left(\mathbb{Q}_{v}\right)\right)$ and $\phi_{v}^{n}$ will satisfy an identity involving orbital integrals. Since we are assuming that $\operatorname{Ram}_{F / \mathbb{Q}} \subseteq \operatorname{Spl}_{F / F_{2}, \mathbb{Q}}$, we can define the transfer at all places $v$ of $\mathbb{Q}$.

It is also possible to define a transfer of pseudo-coefficients at infinity. Consider $\left(G_{\vec{n}}, s_{\vec{n}}, \eta_{\vec{n}}\right) \in \mathcal{E}^{\text {ell }}\left(G_{n}\right)$, which is also an endoscopic triple for $G$. Fix real elliptic maximal tori $T \subset G$ and $T_{G_{\vec{n}}} \subset G_{\vec{n}}$ together with an $\mathbb{R}$-isomorphism $j: T_{G_{\vec{n}}} \stackrel{\sim}{\rightarrow} T$. Also fix a Borel subgroup $B$ of $G$ over $\mathbb{C}$ containing $T_{\mathbb{C}}$. Shelstad defined the transfer factor $\Delta_{j, B}$, see [She].

Let $\xi$ be an irreducible algebraic representation of $G_{\mathbb{C}}$. Define $\chi_{\xi}: A_{G, \infty} \rightarrow \mathbb{C}$ to be the restriction of $\xi$ to $A_{G, \infty}$ (the connected component of the identity in the $\mathbb{R}$-points of the maximal $\mathbb{Q}$-split torus in the center of $G$ ). Choose $K_{\infty} \subset G(\mathbb{R})$ 
to be a maximal compact subgroup (admissible in the sense of [Art]) and define

$$
q(G)=\frac{1}{2} \operatorname{dim}\left(G(\mathbb{R}) / K_{\infty} A_{G, \infty}\right)=2 n-2
$$

For each $\pi \in \Pi_{\text {disc }}\left(G(\mathbb{R}), \xi^{\vee}\right)$ there exists $\phi_{\pi} \in C_{c}^{\infty}\left(G(\mathbb{R}), \chi_{\xi}\right)$ a pseudocoefficient for $\pi$. Any discrete $L$-parameter $\varphi_{G_{\vec{n}}}$ such that $\tilde{\eta}_{\vec{n}} \varphi_{G_{\vec{n}}} \sim \varphi_{\xi}$ corresponds to an $L$-packet of the form $\Pi_{\text {disc }}\left(G_{\vec{n}}(\mathbb{R}), \xi\left(\varphi_{G_{\vec{n}}}\right)^{\vee}\right)$. Define

$$
\begin{gathered}
\phi_{G_{\vec{n}}, \xi\left(\varphi_{G_{\vec{n}}}\right)}:=\frac{1}{\left|\Pi\left(\varphi_{\vec{n}}\right)\right|} \sum_{\pi_{G_{\vec{n}}}} \phi_{\pi_{G_{\vec{n}}}} \text { and } \\
\phi_{\pi}^{G_{\vec{n}}}:=(-1)^{q(G)} \sum_{\tilde{\eta} \varphi_{G_{\vec{n}} \sim \varphi_{\xi}}}\left\langle a_{\omega_{*}\left(\varphi_{G_{\vec{n}}, \xi}\right) \omega_{\pi}}, s\right\rangle \operatorname{det}\left(\omega_{*}\left(\varphi_{G_{\vec{n}}, \xi}\right)\right) \cdot \phi_{G_{\vec{n}}, \xi\left(\varphi_{G_{\vec{n}}}\right)} .
\end{gathered}
$$

Then $\phi_{\pi}^{G_{\vec{n}}}$ is a $\Delta_{j, B^{-}}$-transfer of $\phi_{\pi}$.

We will now review the base change for the groups $G_{\vec{n}}$ and $\mathbb{G}_{\vec{n}}$. Define the group

$$
\mathbb{G}_{\vec{n}}^{+}:=\left(R_{E / \mathbb{Q}} G L_{1} \times R_{F / \mathbb{Q}} G L_{\vec{n}}\right) \rtimes\{1, \theta\},
$$

where $\theta(\lambda, g) \theta^{-1}=\left(\lambda^{c}, \lambda^{c} g^{\#}\right)$ and $g^{\#}=\Phi_{\vec{n}}{ }^{t} g^{c} \Phi_{\vec{n}}^{-1}$. If we denote by $\mathbb{G}_{\vec{n}}^{0}$ and $\mathbb{G}_{\vec{n}}^{0} \theta$ the cosets of $\{1\}$ and $\{\theta\}$ in $\mathbb{G}_{\vec{n}}^{+}$then $\mathbb{G}_{\vec{n}}^{+}=\mathbb{G}_{\vec{n}}^{0} \amalg \mathbb{G}_{\vec{n}}^{0} \theta$. There is a natural $\mathbb{Q}$-isomorphism $\mathbb{G}_{\vec{n}} \stackrel{\sim}{\rightarrow} \mathbb{G}_{\vec{n}}^{0}$ which extends to

$$
\mathbb{G}_{\vec{n}} \rtimes \operatorname{Gal}(E / \mathbb{Q}) \stackrel{\sim}{\rightarrow} \mathbb{G}_{\vec{n}}^{+}
$$

so that $c \in \operatorname{Gal}(E / \mathbb{Q})$ maps to $\theta$.

Let $v$ be a place of $\mathbb{Q}$. A representation $\Pi_{v} \in \operatorname{Irr}\left(\mathbb{G}_{\vec{n}}\left(\mathbb{Q}_{v}\right)\right)$ is called $\theta$-stable if $\Pi_{v} \simeq \Pi_{v} \circ \theta$ as representations of $\mathbb{G}_{\vec{n}}\left(\mathbb{Q}_{v}\right)$. If that is the case, then we can choose an operator $A_{\Pi_{v}}$ on the representation space of $\Pi_{v}$ which induces $\Pi_{v} \stackrel{\sim}{\rightarrow} \Pi_{v} \circ \theta$ and which satisfies $A_{\Pi_{v}}^{2}=$ id. Such an operator is called normalized and it is pinned down up to sign. We can similarly define the notion of $\theta$-stable for 
$\Pi^{\mathfrak{S}} \in \operatorname{Irr}\left(\mathbb{G}_{\vec{n}}\left(\mathbb{A}^{\mathfrak{S}}\right)\right)$ and a corresponding intertwining operator $A_{\Pi \mathfrak{S}}$ for any finite set $\mathfrak{S}$ of places of $\mathbb{Q}$. There is a correspondence between $\theta$-stable representations of $\mathbb{G}_{\vec{n}}\left(\mathbb{Q}_{v}\right)$ together with a normalized intertwining operator and representations of $\mathbb{G}_{\vec{n}}^{+}\left(\mathbb{Q}_{v}\right)$. We also mention that in order for a representation $\Pi \in \operatorname{Irr}\left(\mathbb{G}_{\vec{n}}(\mathbb{A})\right)$ to be $\theta$-stable it is necessary and sufficient that $\Pi=\psi \otimes \Pi^{1}$ satisfy

- $\left(\Pi^{1}\right)^{\vee} \simeq \Pi^{1} \circ c$, and

- $\prod_{i=1}^{r} \psi_{i}=\psi^{c} / \psi$ where $\psi_{\Pi^{1}}=\psi_{1} \otimes \cdots \otimes \psi_{r}$ is the central character of $\Pi^{1}$. Now we shall discuss BC-matching functions. It is possible to construct for each finite place $v$ of $\mathbb{Q}$ and $f_{v} \in C_{c}^{\infty}\left(\mathbb{G}_{\vec{n}}\left(\mathbb{Q}_{v}\right)\right)$ a function $\phi_{v} \in C_{c}^{\infty}\left(G_{\vec{n}}\left(\mathbb{Q}_{v}\right)\right)$, which is the BC-transfer of $f_{v}$. The transfer can be described concretely in the cases $v \in \operatorname{Unr}_{F / \mathbb{Q}}$ and $v \in \operatorname{Spl}_{F / F_{2}, \mathbb{Q}}$, except that in the case $v \in \operatorname{Unr}_{F / \mathbb{Q}}$ we have the condition that $f_{v}$ must be unramified. Moreover, we also have an explicit map

$$
B C_{\vec{n}}: \operatorname{Irr}^{(\mathrm{ur})}\left(G_{\vec{n}}\left(\mathbb{Q}_{v}\right)\right) \rightarrow \operatorname{Irr}^{(\mathrm{ur}) \theta-\mathrm{st}}\left(\mathbb{G}_{\vec{n}}\left(\mathbb{Q}_{v}\right)\right)
$$

where the representations must be unramified in the case $v \in \mathrm{Unr}_{F / \mathbb{Q}}$ and where there is no restriction in the case $v \in \operatorname{Spl}_{F / F_{2}, \mathbb{Q}}$. There are normalized operators $A_{\Pi_{v}}^{0}: \Pi_{v} \stackrel{\sim}{\rightarrow} \Pi_{v} \circ \theta$ such that if $\Pi_{v}=B C_{\vec{n}}\left(\pi_{v}\right)$ and $\phi_{v}$ and $f_{v}$ are BC-matching functions then

$$
\operatorname{tr}\left(\Pi_{v}\left(f_{v}\right) A_{\Pi_{v}}^{0}\right)=\operatorname{tr} \pi_{v}\left(\phi_{v}\right)
$$

Note that left side of the above equality computes the trace of $f_{v} \theta$, the function on $\mathbb{G}_{\vec{n}} \theta$ obtained from $f_{v}$ via translation by $\theta$.

The next step is to consider the base change at $\infty$. Let $\xi_{\vec{n}}$ be an irreducible algebraic representation of $G_{\vec{n}, \mathbb{C}}$. Consider the natural isomorphism

$$
\mathbb{G}_{\vec{n}}(\mathbb{C}) \simeq G_{\vec{n}}(\mathbb{C}) \times G_{\vec{n}}(\mathbb{C}) .
$$


We can define a representation $\Xi_{\vec{n}}$ of $\mathbb{G}_{\vec{n}}$ by $\Xi_{\vec{n}}:=\xi_{\vec{n}} \otimes \xi_{\vec{n}}$. It is possible to find an irreducible, $\theta$-stable, generic unitary representation $\Pi_{\Xi_{\vec{n}}} \in \operatorname{Irr}\left(\mathbb{G}_{\vec{n}}(\mathbb{R}), \chi_{\xi_{\vec{n}}}^{-1}\right)$ together with a normalized operator $A_{\Pi_{\Xi_{\vec{n}}}^{0}}$ and a function $f_{\mathbb{G}_{\vec{n}}, \Xi_{\vec{n}}} \in C_{c}^{\infty}\left(\mathbb{G}(\mathbb{R}), \chi_{\xi_{\vec{n}}}\right)$ such that

- $\Pi_{\Xi_{\vec{n}}}$ is the base change of the $L$-packet $\Pi_{\operatorname{disc}}\left(G_{\vec{n}}(\mathbb{R}), \xi_{\vec{n}}^{\vee}\right)$,

- $\operatorname{tr}\left(\Pi_{\Xi_{\vec{n}}}\left(f_{\mathbb{G}_{\vec{n}}, \Xi_{\vec{n}}}\right) \circ A_{\Pi_{\Xi_{\vec{n}}}}^{0}\right)=2$ and

- $f_{\mathbb{G}_{\vec{n}}, \Xi_{\vec{n}}}$ and $\phi_{G_{\vec{n}}, \xi_{\vec{n}}}$ are BC-matching functions (where $\phi_{G_{\vec{n}}, \xi_{\vec{n}}}$ is defined as a pseudocoefficient for the $L$-packet $\Pi_{\operatorname{disc}}\left(G_{\vec{n}}(\mathbb{R}), \xi_{\vec{n}}^{\vee}\right)$.

The transfer for $\tilde{\zeta}_{n_{1}, n_{2}}$ can be defined explicitly since the groups $\mathbb{G}_{\vec{n}}$ are essentially products of general linear groups. It can be checked that for all finite places $v$ of $\mathbb{Q}$ the transfers are compatible. For $v=\infty$ we have the compatibility relation on the representation-theoretic side follows directly from the commutative diagram of $L$-morphisms.

Now we shall describe the transfer factors $\Delta_{v}(,)_{G_{\vec{n}}}^{G}$. At $v \neq \infty$ we can choose

$$
\Delta_{v}(,)_{G_{\vec{n}}}^{G}=\Delta_{v}^{0}(,)_{G_{\vec{n}}}^{G_{n}}
$$

via the fixed isomorphism $G \times \mathbb{Q} \mathbb{A}^{\infty} \simeq G_{n} \times_{\mathbb{Q}} \mathbb{A}^{\infty}$. We choose the unique $\Delta_{\infty}(,)_{G_{\vec{n}}}^{G}$ such that the product formula

$$
\prod_{v} \Delta_{v}\left(\gamma_{G_{\vec{n}}}, \gamma\right)_{G_{\vec{n}}}^{G}=1
$$

holds for any $\gamma \in G(\mathbb{Q})$ semisimple and $\gamma_{G_{\vec{n}}} \in G_{\vec{n}}(\mathbb{A})$ a $\left(G, G_{\vec{n}}\right)$-regular semisimple element such that $\gamma$ and $\gamma_{G_{\vec{n}}}$ have matching stable conjugacy classes. Let $e_{\vec{n}}\left(\Delta_{\infty}\right) \in \mathbb{C}^{\times}$denote the constant for which

$$
\Delta_{\infty}\left(\gamma_{G_{\vec{n}}}, \gamma\right)_{G_{\vec{n}}}^{G}=e_{\vec{n}}\left(\Delta_{\infty}\right) \Delta_{j, B}\left(\gamma_{G_{\vec{n}}}, \gamma\right)
$$


holds. Note that for $\vec{n}=(n), e_{\vec{n}}\left(\Delta_{\infty}\right)=1$.

Let $\phi^{\infty, p} \cdot \phi_{p}^{\prime} \in C_{c}^{\infty}\left(G\left(\mathbb{A}^{\infty, p}\right) \times J^{\left(h_{1}, h_{2}\right)}\left(\mathbb{Q}_{p}\right)\right)$ be a complex-valued acceptable function. (For a definition of the notion of acceptable function, see Definition 6.2 of [Sh1]). For each $G_{\vec{n}} \in \mathcal{E}^{\text {ell }}(G)$ we define the function $\phi_{\mathrm{Ig}}^{\vec{n}}$ on $G_{\vec{n}}(\mathbb{A})$ (assuming that $\left.\phi^{\infty, p}=\prod_{v \neq p, \infty} \phi_{v}\right)$. For $v \neq p, \infty$, we take $\phi_{\mathrm{Ig}, v}^{\vec{n}} \in C_{c}^{\infty}\left(G_{\vec{n}}\left(\mathbb{Q}_{v}\right)\right)$ to be the $\Delta_{v}(,)_{G_{\vec{n}}}^{G}$-transfer of $\phi_{v}$. We take

$$
\phi_{\mathrm{Ig}, \infty}^{\vec{n}}:=e_{\vec{n}}\left(\Delta_{\infty}\right) \cdot(-1)^{q(G)}\left\langle\mu_{h}, s_{\vec{n}}\right\rangle \sum_{\varphi_{\vec{n}}} \operatorname{det}\left(\omega_{*}\left(\varphi_{G_{\vec{n}}}\right)\right) \cdot \phi_{G_{\vec{n}}, \xi\left(\varphi_{\vec{n}}\right)},
$$

where $\varphi_{\vec{n}}$ runs over $L$-parameters such that $\tilde{\eta}_{\vec{n}} \varphi_{\vec{n}} \sim \varphi_{\xi}$ and $\xi\left(\varphi_{\vec{n}}\right)$ is the algebraic representation of $G_{\vec{n}, \mathbb{C}}$ such that the $L$-packet associated to $\varphi_{\vec{n}}$ is $\Pi_{\text {disc }}\left(G_{\vec{n}}(\mathbb{R}), \xi\left(\varphi_{\vec{n}}\right)^{\vee}\right)$.

We also take

$$
\phi_{\mathrm{Ig}, p}^{\vec{n}} \in C_{c}^{\infty}\left(G_{\vec{n}}\left(\mathbb{Q}_{p}\right)\right)
$$

to be the function constructed from $\phi_{p}^{\prime}$ in section 6.3 of [Sh2]. We shall summarize the construction of $\phi_{\mathrm{Ig}, p}^{\vec{n}}$ in the case $\vec{n}=(n)$. By definition (see the formula above Lemma 6.5 of [Sh2])

$$
\phi_{\mathrm{Ig}, p}^{\vec{n}}=\sum_{\left(M_{G_{n}}, s_{G_{n}}, \eta_{G_{n}}\right)} c_{M_{G_{n}}} \cdot \tilde{\phi}_{p}^{M_{G_{n}}},
$$

where the sum is taken over $G$-endoscopic triples for $J^{\left(h_{1}, h_{2}\right)}$. The set $\mathcal{I}\left(M_{G_{n}}, G_{n}\right)$ (which can be identified with a set of cosets of $\operatorname{Out}\left(M_{G_{n}}, s_{G_{n}}, \eta_{G_{n}}\right)$ ) consists of only one element in our case, so we suppress the index $i \in \mathcal{I}\left(M_{G_{n}}, G_{n}\right)$ in $\tilde{\phi}_{p}^{M_{G_{n}}, i}$. Each $\tilde{\phi}_{p}^{M_{G_{n}}} \in C_{c}^{\infty}\left(G_{n}\left(\mathbb{Q}_{p}\right)\right)$ is constructed from a function $\phi_{p}^{M_{G_{n}}} \in$ $C_{c}^{\infty}\left(M_{G_{n}}\left(\mathbb{Q}_{p}\right)\right)$ which is a $\Delta_{p}(,)_{M_{G_{n}}}^{J^{\left(h_{1}, h_{2}\right)}}$-transfer of a normalized $\phi_{p}^{\prime}$.

The following proposition is Theorem 7.2 of [Sh2].

Proposition 6.0.6. If $\phi^{\infty, p} \cdot \phi_{p}^{\prime} \in C_{c}^{\infty}\left(G\left(\mathbb{A}^{\infty, p}\right) \times J^{\left(h_{1}, h_{2}\right)}\left(\mathbb{Q}_{p}\right)\right)$ is acceptable, 
then

$\operatorname{tr}\left(\phi^{\infty, p} \cdot \phi_{p}^{\prime} \mid \iota_{l} H_{c}\left(\operatorname{Ig}^{\left(h_{1}, h_{2}\right)}, \mathcal{L}_{\xi}\right)\right)=(-1)^{h_{1}+h_{2}}\left|\operatorname{ker}^{1}(\mathbb{Q}, G)\right| \sum_{G_{\vec{n}}} \iota\left(G, G_{\vec{n}}\right) S T_{e}^{G_{\vec{n}}}\left(\phi_{\mathrm{Ig}}^{\vec{n}}\right)$

where the sum runs over the set $\mathcal{E}^{\mathrm{ell}}(G)$ of elliptic endoscopic triples $\left(G_{\vec{n}}, s_{\vec{n}}, \eta_{\vec{n}}\right)$.

Remark 6.0.7. Theorem 7.2 of [Sh2] is proved under the "unramified hypothesis", however, the only place where this hypothesis is needed is in the proof of Lemma 11.1 of [Sh1]. Lemma 5.2.1 provides an alternative to the proof of Lemma 11.1 of [Sh1] in our situation, so the results of [Sh1] and [Sh2] carry over. For details, see the discussion in the beginning of Section 5.2 of [Sh3]. The sign $(-1)^{h_{1}+h_{2}}$ does not show up in the statement of the theorem in [Sh2], but we need to include it because our convention for the alternating sum of the cohomology differs from the usual one by $(-1)^{h_{1}+h_{2}}$.

The constants $\iota\left(G, G_{\vec{n}}\right)=\tau(G) \tau\left(G_{\vec{n}}\right)^{-1}\left|\operatorname{Out}\left(G_{\vec{n}}, s_{\vec{n}}, \eta_{\vec{n}}\right)\right|^{-1}$ can be computed explicitly. We mention that

$$
\left|\operatorname{Out}\left(G_{\vec{n}}, s_{\vec{n}}, \eta_{\vec{n}}\right)\right|= \begin{cases}2 & \text { if } \vec{n}=\left(\frac{n}{2}, \frac{n}{2}\right) \\ 1 & \text { otherwise. }\end{cases}
$$

We also have by Corollary 4.7 of [Sh3] the relation

$$
I_{\text {geom }}^{\mathbb{G}_{\vec{n}} \theta}(f \theta)=\tau\left(G_{\vec{n}}\right)^{-1} \cdot S T_{e}^{G_{\vec{n}}}(\phi)
$$

when $\phi$ and $f$ are BC-matching functions, i.e.

$$
\phi=\phi^{\mathfrak{S}} \cdot \phi_{\mathfrak{S}_{\text {fin }}} \cdot \phi_{G_{\vec{n}}, \xi} \text { and } f=f^{\mathfrak{S}} \cdot f_{\mathfrak{S}_{\text {fin }}} f_{G_{\vec{n}}, \Xi},
$$

with $\phi^{\mathfrak{S}}$ a BC-transfer of $f^{\mathfrak{S}}$ and $\phi_{\mathfrak{S}_{\text {fin }}}$ a BC-transfer of $f_{\mathfrak{S}_{\text {fin }}}$. Thus, assuming 
that for each $\vec{n}$ there exists $f^{\vec{n}}$ such that $\phi_{\mathrm{Ig}}^{\vec{n}}$ and $f^{\vec{n}}$ are BC-matching, we can write

$$
\operatorname{tr}\left(\phi^{\infty, p} \cdot \phi_{p}^{\prime} \mid \iota_{l} H_{c}\left(\operatorname{Ig}^{\left(h_{1}, h_{2}\right)}, \mathcal{L}_{\xi}\right)\right)=\left|\operatorname{ker}^{1}(\mathbb{Q}, G)\right| \cdot \tau(G) \sum_{\mathbb{G}_{\vec{n}}} \epsilon_{\vec{n}} I_{\text {geom }}^{\mathbb{G}_{\vec{n}} \theta}\left(f^{\vec{n}} \theta\right)
$$

where $\epsilon_{\vec{n}}=\frac{1}{2}$ if $\vec{n}=\left(\frac{n}{2}, \frac{n}{2}\right)$ or 1 otherwise.

Furthermore, the twisted trace formula by Arthur, is an equality between

$$
I_{\mathrm{spec}}^{\mathbb{G}_{\vec{n}} \theta}(f \theta)=I_{\text {geom }}^{\mathbb{G}_{\vec{n}} \theta}(f \theta)
$$

By combining Proposition 4.8 and Corollary 4.14 of [Sh3] we can compute $I_{\mathrm{spec}}^{\mathbb{G}_{\vec{n}} \theta}(f \theta)$ as

$$
\sum_{M} \frac{\left|W_{M}\right|}{\left|W_{\mathbb{G}_{\vec{n}}}\right|}\left|\operatorname{det}\left(\Phi_{\vec{n}}^{-1} \theta-1\right)_{\mathfrak{a}_{M}^{\mathbb{G}_{\vec{n}} \theta}}\right|^{-1} \sum_{\Pi_{M}} \operatorname{tr}\left(\mathrm{n}-\operatorname{Ind}_{Q}^{\mathbb{G}_{\vec{n}}}\left(\Pi_{M}\right)_{\xi}(f) \circ A^{\prime}{\mathrm{n}-\operatorname{Ind}_{Q}^{\mathbb{G}_{\vec{n}}}\left(\Pi_{M}\right)_{\xi}}^{\prime}\right.
$$

where $M$ runs over $\mathbb{Q}$-Levi subgroups of $\mathbb{G}_{\vec{n}}$ containing a fixed minimal Levi and $Q$ is a parabolic containing $M$ as a Levi. The rest of the notation is defined on pages 31 and 32 of [Sh3]. Note that $A_{\mathrm{n}-\operatorname{Ind}_{Q}^{\prime} \mathbb{G}_{(}\left(\Pi_{M}\right) \xi}$ is a normalized intertwining operator for $\mathrm{n}-\operatorname{Ind}_{Q}^{\mathbb{G}_{\vec{n}}}\left(\Pi_{M}\right)_{\xi}$.

We will be particularly interested in making the above formula explicit when $\vec{n}=(n)$. In that case, $I_{\mathrm{spec}}^{\mathbb{G} \vec{n} \theta}(f \theta)$ is a sum of

$$
\frac{1}{2} \sum_{\Pi^{\prime}} \operatorname{tr}\left(\Pi_{\xi}^{\prime}(f) A_{\Pi_{\xi}^{\prime}}^{\prime}\right)
$$

where $\Pi^{\prime}$ runs over $\theta$-stable subrepresentations of $R_{\mathbb{G}_{n}, \text { disc }}$, and

$$
\sum_{M \subsetneq \mathbb{G}_{n}} \frac{\left|W_{M}\right|}{\left|W_{\mathbb{G}_{n}}\right|}\left|\operatorname{det}\left(\Phi_{n}^{-1} \theta-1\right)_{\mathfrak{a}_{M}^{\mathbb{G}_{n} \theta}}\right|^{-1} \sum_{\Pi_{M}^{\prime}} \operatorname{tr}\left(\mathrm{n}-\operatorname{Ind}_{Q}^{\mathbb{G}_{n}}\left(\Pi_{M}^{\prime}\right)_{\xi}(f) \circ A_{\mathrm{n}-\operatorname{Ind}_{Q}^{\prime} \mathbb{G}_{n}\left(\Pi_{M}^{\prime}\right)_{\xi}}\right),
$$

where $\Pi_{M}^{\prime}$ runs over $\Phi_{n}^{-1} \theta$-stable subrepresentations of $R_{M, \text { disc }}$. 
Consider the finite set $\mathcal{E}_{p}^{\text {eff }}\left(J^{\left(h_{1}, h_{2}\right)}, G, G_{\vec{n}}\right)$ consisting of certain isomorphism classes of $G$-endoscopic triples $\left(M_{G_{\vec{n}}}, s_{\vec{n}}, \eta_{\vec{n}}\right)$ for $J^{\left(h_{1}, h_{2}\right)}$. This set is defined in section 6.2 of [Sh2]. Let $c_{M_{G_{\vec{n}}}} \in\{ \pm 1\}$ be the constant assigned to each triple in [Sh2]. If $b$ is the isocrystal corresponding to $\left(h_{1}, h_{2}\right)$, let $M^{\left(h_{1}, h_{2}\right)}\left(\mathbb{Q}_{p}\right)$ be the centralizer of $\nu_{G}(b)$. The isocrystal $b$ can be described as $\left(b_{p, 0}, b_{\mathfrak{p}_{1}}, \ldots, b_{\mathfrak{p}_{r}}\right)$ where $b_{\mathfrak{p}_{i}}$ has slopes 0 and $\frac{1}{n-h_{i}}$ for $i=1,2$ and slope 0 for $i>2$. Then $M^{\left(h_{1}, h_{2}\right)}$ is a $\mathbb{Q}_{p}$-rational Levi subgroup of $G$. We will define a group morphism

$$
\mathrm{n}-\operatorname{Red}_{\vec{n}}^{\left(h_{1}, h_{2}\right)}: \operatorname{Groth}\left(G_{\vec{n}}\left(\mathbb{Q}_{p}\right) \rightarrow \operatorname{Groth}\left(J^{\left(h_{1}, h_{2}\right)}\left(\mathbb{Q}_{p}\right)\right)\right.
$$

as the composition of the following maps

$$
\begin{gathered}
\operatorname{Groth}\left(G_{\vec{n}}\left(\mathbb{Q}_{p}\right)\right) \rightarrow \bigoplus_{\left(M_{G_{\vec{n}}}, s_{G_{\vec{n}}}, \eta_{\left.G_{\vec{n}}\right)}\right.} \operatorname{Groth}\left(M_{G_{\vec{n}}}\left(\mathbb{Q}_{p}\right)\right) \stackrel{\oplus \tilde{\eta}_{G_{\vec{n}}, *}}{\longrightarrow} \operatorname{Groth}\left(M^{\left(h_{1}, h_{2}\right)}\left(\mathbb{Q}_{p}\right)\right) \\
\stackrel{L J_{J\left(h_{1}, h_{2}\right)}^{M\left(h_{2}, h_{2}\right)}}{\longrightarrow} \operatorname{Groth}\left(J^{\left(h_{1}, h_{2}\right)}\left(\mathbb{Q}_{p}\right)\right) .
\end{gathered}
$$

The sum runs over $\left(M_{G_{\vec{n}}}, s_{\vec{n}}, \eta_{\vec{n}}\right) \in \mathcal{E}_{p}^{\text {eff }}\left(J^{\left(h_{1}, h_{2}\right)}, G, G_{\vec{n}}\right)$. The first map is the direct sum of maps $\operatorname{Groth}\left(G_{\vec{n}}\left(\mathbb{Q}_{p}\right)\right) \rightarrow \operatorname{Groth}\left(M_{G_{\vec{n}}}\left(\mathbb{Q}_{p}\right)\right)$ which are given by $\oplus_{i} c_{M_{G_{\vec{n}}}} \cdot J_{P\left(i M_{G_{\vec{n}}}\right)}^{G_{\vec{n}}}$ op , where $i \in \mathcal{I}\left(M_{G_{\vec{n}}}, G_{\vec{n}}\right)$ is a $\mathbb{Q}_{p}$-embedding $M_{G_{\vec{n}}} \hookrightarrow G_{\vec{n}}$ and $P\left(i M_{G_{\vec{n}}}\right)$ is a parabolic subgroup of $G_{\vec{n}}$ which contains $i\left(M_{G_{\vec{n}}}\right)$ as a Levi subgroup. The map $\tilde{\eta}_{G_{\vec{n}}, *}$ is functorial transfer with respect to the $L$-morphism $\tilde{\eta}_{G_{\vec{n}}}$. The third map, $L J_{J^{\left(h_{1}, h_{2}\right)}}^{M^{\left(h_{1}, h_{2}\right)}}$ is the Jacquet-Langlands map on Grothendieck groups. We also define

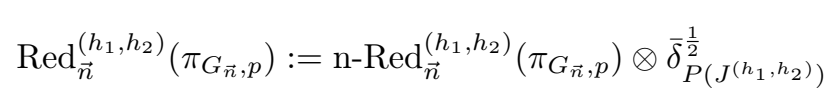

We can describe all the groups and maps above very explicitly in the case $\vec{n}=$ $(n)$. Indeed, $\mathcal{E}_{p}^{\mathrm{eff}}\left(J^{\left(h_{1}, h_{2}\right)}, G, G_{n}\right)$ has a unique isomorphism class represented 
by

$$
\left(M_{G_{n}}, s_{G_{n}}, \eta_{G_{n}}\right)=\left(M^{\left(h_{1}, h_{2}\right)}, 1, \mathrm{id}\right) .
$$

The set $\mathcal{I}\left(M_{G_{n}}, G_{n}\right)$ is also a singleton in this case, so we suppress $i$ everywhere. This means that we can also take $\tilde{\eta}_{G_{n}}=\mathrm{id}$ and $\tilde{\eta}_{G_{n}, *}=\mathrm{id}$ and by Remark 6.4 of [Sh2], we may also take $c_{M_{G_{n}}}=e_{p}\left(J^{\left(h_{1}, h_{2}\right)}\right)$, which is the Kottwitz sign of the $\mathbb{Q}_{p}$-group $J^{\left(h_{1}, h_{2}\right)}$. There are isomorphisms

$$
\begin{gathered}
G\left(\mathbb{Q}_{p}\right) \simeq \mathbb{Q}_{p}^{\times} \times G L_{n}\left(F_{\mathfrak{p}_{1}}\right) \times G L_{n}\left(F_{\mathfrak{p}_{2}}\right) \times \prod_{i>2} G L_{n}\left(F_{\mathfrak{p}_{i}}\right), \\
M^{\left(h_{1}, h_{2}\right)}\left(\mathbb{Q}_{p}\right) \simeq \mathbb{Q}_{p}^{\times} \times\left(G L_{n-h_{1}}\left(F_{\mathfrak{p}_{1}}\right) \times G L_{h_{1}}\left(F_{\mathfrak{p}_{1}}\right)\right) \times\left(G L_{n-h_{2}}\left(F_{\mathfrak{p}_{2}}\right) \times G L_{h_{2}}\left(F_{\mathfrak{p}_{2}}\right)\right) \\
\times \prod_{i>2} G L_{n}\left(F_{\mathfrak{p}_{i}}\right), \\
J^{\left(h_{1}, h_{2}\right)}\left(\mathbb{Q}_{p}\right) \simeq \mathbb{Q}_{p}^{\times} \times\left(D_{F_{\mathfrak{p}_{1}}, \frac{1}{n-h_{1}}}^{\times} \times G L_{h_{1}}\left(F_{\mathfrak{p}_{1}}\right)\right) \times\left(D_{F_{\mathfrak{p}_{2}}, \frac{1}{n-h_{2}}}^{\times} \times G L_{h_{2}}\left(F_{\mathfrak{p}_{2}}\right)\right) \times \prod_{i>2} G L_{n}\left(F_{\mathfrak{p}_{i}}\right) .
\end{gathered}
$$

Thus, $e_{p}\left(J^{\left(h_{1}, h_{2}\right)}\right)=(-1)^{2 n-2-h_{1}-h_{2}}$. If we write $\pi_{p}=\pi_{p, 0} \otimes\left(\otimes_{i} \pi_{\mathfrak{p}_{i}}\right)$, then we have

$\operatorname{Red}_{n}^{\left(h_{1}, h_{2}\right)}\left(\pi_{p}\right)=(-1)^{h_{1}+h_{2}} \pi_{p, 0} \otimes \operatorname{Red}^{n-h_{1}, h_{1}}\left(\pi_{\mathfrak{p}_{1}}\right) \otimes \operatorname{Red}^{n-h_{2}, h_{2}}\left(\pi_{\mathfrak{p}_{2}}\right) \otimes\left(\otimes_{i>2} \pi_{\mathfrak{p}_{i}}\right)$.

Lemma 6.0.8. For any $\pi_{p} \in \operatorname{Groth}\left(G_{n}\left(\mathbb{Q}_{p}\right)\right)$

$$
\operatorname{tr} \pi_{p}\left(\phi_{\mathrm{Ig}, p}^{n}\right)=\operatorname{tr}\left(\operatorname{Red}_{n}^{\left(h_{1}, h_{2}\right)}\left(\pi_{p}\right)\right)\left(\phi_{p}^{\prime}\right)
$$

Proof. Set $M=M_{G_{n}}$. We know that $\phi_{\mathrm{Ig}, p}^{n}=e_{p}\left(J^{\left(h_{1}, h_{2}\right)}\right) \cdot \tilde{\phi}_{p}^{M}$. By Lemma 3.9 of [Sh2],

$$
\operatorname{tr} \pi_{p}\left(\tilde{\phi}_{p}^{M}\right)=\operatorname{tr}\left(J_{P_{M}^{\text {op }}}^{G_{n}}\left(\pi_{p}\right)\right)\left(\phi_{p}^{M}\right) .
$$

Here $\phi_{p}^{M}$ is a $\Delta_{p}(,)_{M}^{J^{\left(h_{1}, h_{2}\right)}} \equiv e_{p}\left(J^{\left(h_{1}, h_{2}\right)}\right)$-transfer of $\phi_{p}^{0}=\phi_{p}^{\prime} \cdot \bar{\delta}_{P\left(J^{\left(h_{1}, h_{2}\right)}\right)}^{\frac{1}{2}}($ by 
remark 6.4 of [Sh2], we have an explicit description of the transfer factor). Let $\pi_{M, p}=J_{P_{M}^{o p}}^{G_{n}}\left(\pi_{p}\right)$.

Note that $M$ is a product of general linear groups and $J^{\left(h_{1}, h_{2}\right)}$ is an inner form of $M$. Lemma 2.18 and Remark 2.19 of [Sh2] ensure that

$\operatorname{tr} \pi_{M, p}\left(\phi_{p}^{M}\right)=\operatorname{tr}\left(L J_{M}^{J^{\left(h_{1}, h_{2}\right)}}\left(\pi_{M, p}\right)\left(\phi_{p}^{0}\right)\right)=\operatorname{tr}\left(L J_{M}^{J^{\left(h_{1}, h_{2}\right)}}\left(\pi_{M, p}\right) \otimes \bar{\delta}_{P\left(J^{\left(h_{1}, h_{2}\right)}\right)}^{\frac{1}{2}}\right)\left(\phi_{p}^{\prime}\right)$.

This concludes the proof.

Lemma 6.0.9. Let $\vec{n}=\left(n_{1}, n_{2}\right)$ with $n_{1} \geq n_{2}>0$. For any $\pi_{p} \in \operatorname{Groth}\left(G_{n_{1}, n_{2}}\left(\mathbb{Q}_{p}\right)\right)$,

$$
\operatorname{tr} \pi_{p}\left(\phi_{\mathrm{Ig}, p}^{\vec{n}}\right)=\operatorname{tr}\left(\operatorname{Red}_{\vec{n}}^{\left(h_{1}, h_{2}\right)}\left(\pi_{p}\right)\right)\left(\phi_{p}^{\prime}\right)
$$

Proof. The proof is based on making explicit the construction of $\phi_{\mathrm{Ig}, p}^{\vec{n}}$ from section 6 of [Sh2] together with the definition of the functor n- $\operatorname{Red}_{\vec{n}}^{\left(h_{1}, h_{2}\right)}$, which is a composition of the following maps:

$$
\begin{aligned}
& \operatorname{Groth}\left(G_{\vec{n}}\left(\mathbb{Q}_{p}\right)\right) \rightarrow \bigoplus_{\left(M_{G_{\vec{n}}}, s_{G_{\vec{n}}}, \eta_{G_{\vec{n}}}\right)} \operatorname{Groth}\left(M_{G_{\vec{n}}}\left(\mathbb{Q}_{p}\right)\right) \stackrel{\oplus \tilde{\eta}_{G_{\vec{n}}, *}}{\longrightarrow} \operatorname{Groth}\left(M^{\left(h_{1}, h_{2}\right)}\left(\mathbb{Q}_{p}\right)\right) \\
& \stackrel{L J_{J}^{M\left(h_{1}, h_{2}\right)}}{\longrightarrow} \operatorname{Groth}\left(J^{\left(h_{1}, h_{2}\right)}\left(\mathbb{Q}_{p}\right)\right) .
\end{aligned}
$$

Recall that

$$
\phi_{\mathrm{Ig}, p}^{\vec{n}}=\sum_{\left(M_{G_{\vec{n}}}, s_{G_{\vec{n}}}, \eta_{G_{\vec{n}}}\right)} \sum_{i} c_{M_{G_{\vec{n}}}} \cdot \tilde{\phi}_{p}^{M_{G_{\vec{n}}}, i}
$$

as functions on $G_{\vec{n}}\left(\mathbb{Q}_{p}\right)$, where the first sum is taken over $\mathcal{E}_{p}^{\text {eff }}\left(J^{\left(h_{1}, h_{2}\right)}, G, G_{\vec{n}}\right)$ and the second sum is taken over $\mathcal{I}\left(M_{G_{\vec{n}}}, G_{\vec{n}}\right)$. By Lemma 3.9 of [Sh2],

$$
\operatorname{tr} \pi_{p}\left(\tilde{\phi}_{p}^{M_{G_{\vec{n}}, i}}\right)=\operatorname{tr}\left(J_{P\left(i M_{G_{\vec{n}}}\right)^{\mathrm{op}}}^{G_{\vec{n}}}\left(\pi_{p}\right)\left(\phi_{p}^{M_{G_{\vec{n}}}}\right)\right.
$$


where $\phi_{p}^{M_{G_{\vec{n}}}} \in C_{c}^{\infty}\left(M_{G_{\vec{n}}}\left(\mathbb{Q}_{p}\right)\right)$ is a $\Delta_{p}(,)_{M_{G_{\vec{n}}}^{\left(h_{1}, h_{2}\right)}}$-transfer of $\phi_{p}^{0}=\phi_{p}^{\prime} \cdot \bar{\delta}_{P\left(J^{\left(h_{1}, h_{2}\right)}\right)}^{\frac{1}{2}}$. Equation 6.1 tells us that

$$
\operatorname{tr} \pi_{p}\left(\phi_{\mathrm{Ig}, p}^{\vec{n}}\right)=\sum_{\left(M_{G_{\vec{n}}}, s_{G_{\vec{n}}}, \eta_{G_{\vec{n}}}\right)} \operatorname{tr}\left(f_{M_{G_{\vec{n}}}}\left(\pi_{p}\right)\right)\left(\phi_{p}^{M_{G_{\vec{n}}}}\right),
$$

where $f_{M_{G_{\vec{n}}}}\left(\pi_{p}\right)=\oplus_{i} c_{M_{G_{\vec{n}}}} J_{P\left(i M_{G_{\vec{n}}}\right)}^{G_{\vec{n}}}\left(\pi_{p}\right)$. The first map in the definition of $\operatorname{Red}_{\vec{n}}^{\left(h_{1}, h_{2}\right)}$ is the direct sum of $f_{M_{G_{\vec{n}}}}$ over all $\left(M_{G_{\vec{n}}}, s_{\vec{n}}, \eta_{\vec{n}}\right)$.

The function $\phi_{p}^{M_{G_{\vec{n}}}}$ is a $\Delta_{p}(,)_{M_{G_{\vec{n}}}}^{M^{\left(h_{1}, h_{2}\right)}}$-transfer of the function $\phi_{p}^{*} \in$ $C_{c}^{\infty}\left(M^{\left(h_{1}, h_{2}\right)}\left(\mathbb{Q}_{p}\right)\right)$ which is itself a transfer of $\phi_{p}^{0}$ via $\Delta_{p}(,)_{M^{\left(h_{1}, h_{2}\right)}}^{J^{\left(h_{1}, h_{2}\right)}} \equiv e_{p}\left(J^{\left(h_{1}, h_{2}\right)}\right)$. (All transfer factors are normalized as in [Sh2].) We will focus on making the $\Delta_{p}(,)_{M_{G_{\vec{n}}}}^{M^{\left(h_{1}, h_{2}\right)}}$-transfer explicit first, for which we need to have a complete description of all endoscopic triples $\left(M_{G_{\vec{n}}}, s_{G_{\vec{n}}}, \eta_{G_{\vec{n}}}\right)$.

We have the following isomorphisms over $\mathbb{Q}_{p}$.

$$
\begin{gathered}
G \simeq G L_{1} \times \prod_{i \geq 1} R_{F_{\mathfrak{p}_{i}} / \mathbb{Q}_{p}} G L_{n} \\
G_{n_{1}, n_{2}} \simeq G L_{1} \times \prod_{i \geq 1} R_{F_{\mathfrak{p}_{i}} / \mathbb{Q}_{p}} G L_{n_{1}, n_{2}} \\
M^{\left(h_{1}, h_{2}\right)} \simeq G L_{1} \times \prod_{i=1}^{2} R_{F_{\mathfrak{p}_{i}} / \mathbb{Q}_{p}} G L_{n-h_{i}, h_{i}} \times \prod_{i>2} R_{F_{\mathfrak{p}_{i}} / \mathbb{Q}_{p}} G L_{n} \\
J^{\left(h_{1}, h_{2}\right)} \simeq G L_{1} \times \prod_{i=1}^{2}\left(D_{F_{\mathfrak{p}_{i}}, \frac{1}{n-h_{i}}}^{\times} \times G L_{h_{i}}\right) \times \prod_{i>2} R_{F_{\mathfrak{p}_{i}} / \mathbb{Q}_{p}} G L_{n} .
\end{gathered}
$$

Consider also the following four groups over $\mathbb{Q}_{p}$, which can be thought of as Levi subgroups of $G_{n_{1}, n_{2}}$ via the block diagonal embeddings.

$$
M_{G_{\vec{n}}, 1}:=G L_{1} \times \prod_{i=1}^{2} R_{F_{\mathfrak{p}_{\mathfrak{i}}} / \mathbb{Q}_{p}} G L_{n-h_{i}, h_{i}-n_{2}, n_{2}} \times \prod_{i>2} R_{F_{\mathfrak{p}_{i}} / \mathbb{Q}_{p}} G L_{n_{1}, n_{2}}
$$


CHAPTER 6. THE COHOMOLOGY OF IGUSA VARIETIES

$$
\begin{gathered}
M_{G_{\vec{n}}, 2}:=G L_{1} \times \prod_{i=1}^{2} R_{F_{\mathfrak{p}_{\mathfrak{i}}} / \mathbb{Q}_{p}} G L_{n-h_{i}, h_{i}-n_{1}, n_{1}} \times \prod_{i>2} R_{F_{\mathfrak{p}_{i}} / \mathbb{Q}_{p}} G L_{n_{1}, n_{2}} \\
M_{G_{\vec{n}}, 3}:=G L_{1} \times \prod_{i=1}^{2} R_{F_{\mathfrak{p}_{\mathfrak{i}}} / \mathbb{Q}_{p}} G L_{n-h_{i}, h_{i}-n_{i}, n_{i}} \times \prod_{i>2} R_{F_{\mathfrak{p}_{i}} / \mathbb{Q}_{p}} G L_{n_{1}, n_{2}} \\
M_{G_{\vec{n}}, 4}:=G L_{1} \times \prod_{i=1}^{2} R_{F_{\mathfrak{p}_{\mathfrak{i}}} / \mathbb{Q}_{p}} G L_{n-h_{i}, h_{i}-n_{3-i}, n_{3-i}} \times \prod_{i>2} R_{F_{\mathfrak{p}_{i}} / \mathbb{Q}_{p}} G L_{n_{1}, n_{2}}
\end{gathered}
$$

Note that we only define $M_{G_{\vec{n}}, j}$ when it makes sense, for example $M_{G_{\vec{n}}, 1}$ is defined only when $h_{i} \geq n_{2}$ for $i=1,2$. We define $\eta_{G_{\vec{n}}, j}: \widehat{M_{G_{\vec{n}}, j}} \rightarrow \widehat{M^{\left(h_{1}, h_{2}\right)}}$ to be the obvious block diagonal embedding. We also let

$$
s_{M_{G_{\vec{n}}, j}}=\left(1,( \pm 1, \pm 1, \pm 1)_{i=1,2},(1,1)_{i>2}\right),
$$

where the signs on the $F_{\mathfrak{p}_{i}}$-component are chosen such that $s_{M_{G_{\vec{n}}}, j}$ is positive on the $G L_{n_{1}}$-block of the $F_{\mathfrak{p}_{i}}$-component and negative on the $G L_{n_{2}}$-block of the $F_{\mathfrak{p}_{i}}$-component.

It is easy to check, as on page 42 of [Sh3], that $\mathcal{E}_{p}^{\text {eff }}\left(J^{\left(h_{1}, h_{2}\right)}, G, G_{\vec{n}}\right)$ consists of those triples $\left(M_{G_{\vec{n}}, j}, s_{G_{\vec{n}}, j}, \eta_{G_{\vec{n}}, j}\right)$ which make sense. For example, if $h_{i}<n_{2}$ for $i=1,2$ then $\mathcal{E}^{\text {eff }}\left(J^{\left(h_{1}, h_{2}\right)}, G, G_{\vec{n}}\right)$ is empty, but if $h_{i} \geq n_{1}$ for $i=1,2$ then $\mathcal{E}^{\mathrm{eff}}\left(J^{\left(h_{1}, h_{2}\right)}, G, G_{\vec{n}}\right)$ consists of four elements. The key point is to notice that for a triple $\left(M_{G_{\vec{n}}}, s_{G_{\vec{n}}}, \eta_{G_{\vec{n}}}\right)$ to lie in $\mathcal{E}^{\text {eff }}\left(J^{\left(h_{1}, h_{2}\right)}, G, G_{\vec{n}}\right)$ it is necessary for $s_{G_{\vec{n}}}$ to transfer to an element of the dual group $\widehat{M^{\left(h_{1}, h_{2}\right)}}=\widehat{J^{\left(h_{1}, h_{2}\right)}}$ which is either +1 or -1 in the $G L_{n-h_{i}}(\mathbb{C})$ block of the $F_{\mathfrak{p}_{i}}$-component.

We can extend $\eta_{G_{\vec{n}}, j}$ to an $L$-morphism $\tilde{\eta}_{G_{\vec{n}}, j}:{ }^{L} M_{G_{\vec{n}}, j} \rightarrow{ }^{L} M^{\left(h_{1}, h_{2}\right)}$ which is compatible with the $L$-morphism $\eta_{\vec{n}}:{ }^{L} G_{\vec{n}} \rightarrow{ }^{L} G$, when we map ${ }^{L} M_{G_{\vec{n}}}, j \stackrel{\tilde{l}_{j}}{\rightarrow}{ }^{L} G_{\vec{n}}$ and ${ }^{L} M^{\left(h_{1}, h_{2}\right)} \stackrel{\tilde{l}}{\rightarrow}{ }^{L} G$ via (a conjugate of) the obvious block diagonal embedding (where we always send the $G L_{n_{1}}$-block to the top left corner and the $G L_{n_{2}}$-block to the bottom right corner). The morphism $\tilde{\eta}_{G_{\vec{n}}, j}$ is defined as on page 42 of 
[Sh3], by sending $z \in W_{\mathbb{Q}_{p}}$ to one of the matrices

$$
\left(\begin{array}{cc}
\varpi(z)^{\epsilon\left(n-n_{1}\right)} I_{n_{1}} & 0 \\
0 & \varpi(z)^{\epsilon\left(n-n_{2}\right)} I_{n_{2}}
\end{array}\right) \text { or }\left(\begin{array}{cc}
\varpi(z)^{\epsilon\left(n-n_{2}\right)} I_{n_{2}} & 0 \\
0 & \varpi(z)^{\epsilon\left(n-n_{1}\right)} I_{n_{1}}
\end{array}\right) \text {, }
$$

on the $F_{\mathfrak{p}_{i}}$-component of $\widehat{M^{\left(h_{1}, h_{2}\right)}}$. (For $i=1,2$, we send $z$ to the first matrix on the $F_{\mathfrak{p}_{i}}$-component if the endoscopic group $M_{G_{\vec{n}}, j}$ at $\mathfrak{p}_{i}$ is $G L_{n-h_{i}, h_{i}-n_{2}, n_{2}}$ and to the second matrix if the component of $M_{G_{\vec{n}}, j}$ at $\mathfrak{p}_{i}$ is $G L_{n-h_{i}, h_{i}-n_{1}, n_{1}}$. For $i>2$, we send $z$ to the first matrix on the $F_{\mathfrak{p}_{i}}$-component.) This map $\tilde{\eta}_{G_{\vec{n}}, j}$ is the unique $L$-morphism which makes the diagram

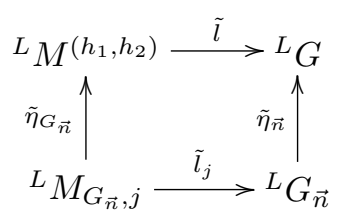

commutative. Thus, the function $\phi_{p}^{M_{G_{\vec{n}}, j}}$ is a transfer of $\phi_{p}^{*}$ with respect to the $L$ morphism $\tilde{\eta}_{G_{\vec{n}}, j}$, so we can define explicitly both $\phi_{p}^{M_{G_{\vec{n}}, j}}$ and the representationtheoretic map $\tilde{\eta}_{M_{G_{\vec{n}}, j} *}: \operatorname{Groth}\left(M_{G_{\vec{n}}, j}\left(\mathbb{Q}_{p}\right)\right) \rightarrow \operatorname{Groth}\left(M^{\left(h_{1}, h_{2}\right)}\left(\mathbb{Q}_{p}\right)\right)$. There exists a unitary character $\chi_{u, j}^{+}: M_{G_{\vec{n}}, j}\left(\mathbb{Q}_{p}\right) \rightarrow \mathbb{C}^{\times}$(defined similarly to the character on page 43 of [Sh3]) such that the Langlands-Shelstad transfer factor with respect to $\tilde{\eta}_{G_{\vec{n}}, j}$ differs from the transfer factor associated to the canonical $L$-morphism by the cocycle associated to $\chi_{u, j}^{+}$. (See section 9 of [Bor] for an explanation of the correspondence between cocycles in $H^{1}\left(W_{\mathbb{Q}_{p}}, Z\left(\widehat{M_{G_{\vec{n}}}, j}\right)\right)$ and characters $\left.M_{G_{\vec{n}}, j}\left(\mathbb{Q}_{p}\right) \rightarrow \mathbb{C}^{\times}.\right)$

We can in fact compute $\chi_{u, j}^{+}$on the different components of $M_{G_{\vec{n}}, j}\left(\mathbb{Q}_{p}\right)$, by keeping in mind that it is the character $M_{G_{\vec{n}}, j}\left(\mathbb{Q}_{p}\right) \rightarrow \mathbb{C}^{\times}$associated to the cocycle in $H^{1}\left(W_{\mathbb{Q}_{p}}, Z\left(\widehat{M_{G_{\vec{n}}}}, j\right)\right.$ which takes the conjugacy class of the standard 
Levi embedding $\widehat{M_{G_{\vec{n}}, j}} \rightarrow \widehat{M^{\left(h_{1}, h_{2}\right)}}$ to that of $\eta_{G_{\vec{n}}, j}$. Thus, we have

$$
\begin{gathered}
\chi_{u, j}^{+}(\lambda)=\varpi_{u}(\lambda)^{-N\left(n_{1}, n_{2}\right)} ; \\
\chi_{u, j}^{+}\left(g_{\mathfrak{p}_{i}, 1}, g_{\mathfrak{p}_{i}, 2}, g_{\mathfrak{p}_{i}, 3}\right)=\left\{\begin{array}{l}
\varpi_{u}\left(N_{F_{\mathfrak{p}_{i}} / E_{u}}\left(\operatorname{det}\left(\left(g_{\mathfrak{p}_{i}, 1} g_{\mathfrak{p}_{i, 2}}\right)^{\epsilon\left(n-n_{1}\right)} g_{\mathfrak{p}_{i}, 3}^{\epsilon\left(n-n_{2}\right)}\right)\right)\right) \\
\varpi_{u}\left(N_{F_{\mathfrak{p}_{i}} / E_{u}}\left(\operatorname{det}\left(\left(g_{\mathfrak{p}_{i}, 1} g_{\mathfrak{p}_{i, 2}}\right)^{\epsilon\left(n-n_{2}\right)} g_{\mathfrak{p}_{i}, 3}^{\epsilon\left(n-n_{1}\right)}\right)\right)\right)
\end{array}\right.
\end{gathered}
$$

when $i=1,2$ and depending on whether $M_{G_{\vec{n}}, j}$ has the group $G L_{n-h_{i}, h_{i}-n_{2}, n_{2}}$ or the group $G L_{n-h_{i}, h_{i}-n_{1}, n_{1}}$ as its $F_{\mathfrak{p}_{i}}$-component; and

$$
\chi_{u, j}^{+}\left(g_{\mathfrak{p}_{i}, 1}, g_{\mathfrak{p}_{i}, 2}\right)=\varpi_{u}\left(N_{\mathfrak{p}_{i} / E_{u}}\left(\operatorname{det}\left(g_{\mathfrak{p}_{i}, 1}^{\epsilon\left(n-n_{1}\right)} g_{\mathfrak{p}_{i}, 2}^{\epsilon\left(n-n_{2}\right)}\right)\right)\right) \text { when } i>2
$$

where $\left(\lambda,\left(g_{\mathfrak{p}_{i}, 1}, g_{\mathfrak{p}_{i}, 2}, g_{\mathfrak{p}_{i}, 3}\right)_{i=1,2},\left(g_{\mathfrak{p}_{i}, 1}, g_{\mathfrak{p}_{i}, 2}\right)_{i>2}\right)$ denotes an element of $M_{G_{\vec{n}}, j}\left(\mathbb{Q}_{p}\right)$. (The value of $\chi_{u, j}^{+}$is in fact the product of the three types of factors above.)

We let $Q_{j}$ be a parabolic subrgroup of $M^{\left(h_{1}, h_{2}\right)}$ containing $M_{G_{\vec{n}}, j}$ as a Levi and if we let $\left(\phi_{p}^{*}\right)^{Q_{j}}$ be the constant term of $\phi_{p}^{*}$ along $Q_{j}$ then we have

$$
\begin{gathered}
\phi_{p}^{M_{G_{\vec{n}}}, j}:=\left(\phi_{p}^{*}\right)^{Q_{j}} \cdot \chi_{u, j}^{+} \text {and } \\
\tilde{\eta}_{G_{\vec{n}}, j *}\left(\pi_{M_{G_{\vec{n}}}, j}\right):=\mathrm{n}-\operatorname{Ind}_{Q_{j}}^{M^{\left(h_{1}, h_{2}\right)}}\left(\pi_{M_{G_{\vec{n}}, j}} \otimes \chi_{u, j}^{+}\right)
\end{gathered}
$$

for any $\pi_{M_{G_{\vec{n}}, j}} \in \operatorname{Irr}_{l}\left(M_{G_{\vec{n}}, j}\left(\mathbb{Q}_{p}\right)\right)$. By Lemma 3.3 of [Sh3]

$$
\operatorname{tr}\left(f_{M_{G_{\vec{n}}, j}}\left(\pi_{p}\right)\right)\left(\phi_{p}^{M_{G_{\vec{n}}, j}}\right)=\operatorname{tr}\left(\tilde{\eta}_{G_{\vec{n}}, j *}\left(f_{M_{G_{\vec{n}}, j}}\left(\pi_{p}\right)\right)\right)\left(\phi_{p}^{*}\right)
$$

The group $J^{\left(h_{1}, h_{2}\right)}$ is an inner form of $M^{\left(h_{1}, h_{2}\right)}$, which is a product of general linear groups. By Lemma 2.18 and Remark 2.19 of [Sh2],

$$
\operatorname{tr}\left(\tilde{\eta}_{G_{\vec{n}}, *}\left(f_{M_{j}}\left(\pi_{p}\right)\right)\right)\left(\phi_{p}^{*}\right)=\operatorname{tr}\left(L J\left(\tilde{\eta}_{G_{\vec{n}}, *}\left(f_{M_{j}}\left(\pi_{p}\right)\right)\right)\right)\left(\phi_{p}^{0}\right)
$$




$$
=\operatorname{tr}\left(L J\left(\tilde{\eta}_{G_{\vec{n}}, *}\left(f_{M_{j}}\left(\pi_{p}\right)\right) \otimes \bar{\delta}_{P\left(J^{\left(h_{1}, h_{2}\right)}\right)}^{\frac{1}{2}}\right)\left(\phi_{p}^{\prime}\right)\right.
$$

where we've abbreviated $M_{G_{\vec{n}}, j}$ by $M_{j}$. Putting together (6.2),(6.3) and (6.4), we get the desired result.

Let $\Xi^{1}$ be the algebraic representation of $\left(\mathbb{G}_{n}\right)_{\mathbb{C}}$ obtained by base change from $\iota_{l} \xi$. Let $\Pi^{1} \simeq \psi \otimes \Pi^{0}$ be an automorphic representation of $\mathbb{G}_{n}(\mathbb{A}) \simeq$ $G L_{1}\left(\mathbb{A}_{E}\right) \times G L_{n}\left(\mathbb{A}_{F}\right)$. Assume that

- $\Pi^{1} \simeq \Pi^{1} \circ \theta$,

- $\Pi_{\infty}^{1}$ is generic and $\Xi^{1}$-cohomological,

- $\operatorname{Ram}_{\mathbb{Q}}(\Pi) \subset \operatorname{Spl}_{F / F_{2}, \mathbb{Q}}$,

- $\Pi^{1}$ is cuspidal.

In particular, $\Pi_{\infty}^{1} \simeq \Pi_{\Xi}$, which was defined above. Let $\mathfrak{S}_{\text {fin }}$ be a finite set of places of $\mathbb{Q}$ such that

$$
\operatorname{Ram}_{F / \mathbb{Q}} \cup \operatorname{Ram}_{\mathbb{Q}}(\varpi) \cup \operatorname{Ram}_{\mathbb{Q}}(\Pi) \cup\{p\} \subset \mathfrak{S}_{\text {fin }} \subset \operatorname{Spl}_{F / F_{2}, \mathbb{Q}}
$$

and let $\mathfrak{S}=\mathfrak{S}_{\text {fin }} \cup\{\infty\}$.

Theorem 6.0.10. Define $C_{G}=\left|\operatorname{ker}^{1}(\mathbb{Q}, G)\right| \cdot \tau(G)$. For each $0 \leq h_{1}, h_{2} \leq n$, the following equality holds in $\operatorname{Groth}\left(\mathbb{G}_{n}\left(\mathbb{A}_{\mathfrak{G}_{\text {fin }} \backslash\{p\}}\right) \times J^{\left(h_{1}, h_{2}\right)}\left(\mathbb{Q}_{p}\right)\right.$.

$$
\begin{gathered}
B C_{\mathfrak{S}_{\text {fin } \backslash\{p\}}\left(H_{c}\left(\operatorname{Ig}^{\left(h_{1}, h_{2}\right)}, \mathcal{L}_{\xi}\right)\right)\left\{\Pi^{1, \mathfrak{S}}\right\}} \\
=C_{G} \cdot e_{0} \cdot(-1)^{h_{1}+h_{2}} \cdot\left[\iota_{l}^{-1} \Pi_{\mathfrak{S}_{\text {fin }} \backslash\{p\}}\right]\left[\operatorname{Red}_{n}^{\left(h_{1}, h_{2}\right)}\left(\pi_{p}\right)\right],
\end{gathered}
$$

where $e_{0}= \pm 1$ is independent of $\left(h_{1}, h_{2}\right)$.

Proof. The proof goes through identically to the proof of the first part of Theorem 6.1 of [Sh3]. We nevertheless give the proof in detail. 
First, we explain the choice of test functions to be used in the trace formula. Let $\left(f^{n}\right)^{\mathfrak{S}} \in \mathcal{H}^{\text {ur }}\left(\mathbb{G}_{n}\left(\mathbb{A}^{\mathfrak{S}}\right)\right)$ and $f_{\mathfrak{S}_{\text {fin }}^{n} \backslash\{p\}} \in C_{c}^{\infty}\left(\mathbb{G}_{n}\left(\mathbb{A}_{\mathfrak{S}_{\text {fin }} \backslash\{p\}}\right)\right)$ be any functions. Let $\phi^{\mathfrak{S}}$ and $\phi_{\mathfrak{S}_{\text {fin }} \backslash\{p\}}$ be the BC-transfers of $\left(f^{n}\right)^{\mathfrak{S}}$ and $\left(f^{n}\right)_{\mathfrak{S}_{\text {fin }} \backslash\{p\}}$ from $\mathbb{G}_{n}$ to $G_{n}$. Let $\phi^{\infty, p}=\phi^{\mathfrak{S}} \phi_{\mathfrak{S}_{\text {fin }} \backslash\{p\}}$ and choose any $\phi_{p}^{\prime} \in C_{c}^{\infty}\left(J^{\left(h_{1}, h_{2}\right)}\left(\mathbb{Q}_{p}\right)\right)$ such that $\phi^{\infty, p} \phi_{p}^{\prime}$ is an acceptable function.

For each $G_{\vec{n}} \in \mathcal{E}^{\text {ell }}(G)$ we construct the function $\phi_{\mathrm{Ig}}^{\vec{n}} \in C_{c}^{\infty}\left(G_{\vec{n}}(\mathbb{A})\right)$ associated to $\phi^{\infty, p} \phi_{p}^{\prime}$ as above. Recall that $\left(\phi_{\mathrm{Ig}}^{\vec{n}}\right)^{\mathfrak{S}}$ and $\left(\phi_{\mathrm{Ig}}^{\vec{n}}\right)_{\mathfrak{S}_{\mathrm{fin}} \backslash\{p\}}$ are the $\Delta(,)_{G_{\vec{n}}}^{G_{n}}$ transfers of $\phi^{\mathfrak{S}}$ and $\phi_{\mathfrak{S}_{\text {fin }} \backslash\{p\}}$. Recall that we take

$$
\phi_{\mathrm{Ig}, \infty}^{\vec{n}}:=e_{\vec{n}}\left(\Delta_{\infty}\right) \cdot(-1)^{q(G)}\left\langle\mu_{h}, s_{\vec{n}}\right\rangle \sum_{\varphi_{\vec{n}}} \operatorname{det}\left(\omega_{*}\left(\varphi_{G_{\vec{n}}}\right)\right) \cdot \phi_{G_{\vec{n}}, \xi\left(\varphi_{\vec{n}}\right)},
$$

where $\varphi_{\vec{n}}$ runs over $L$-parameters such that $\tilde{\eta}_{\vec{n}} \varphi_{\vec{n}} \sim \varphi_{\xi}$ and $\xi\left(\varphi_{\vec{n}}\right)$ is the algebraic representation of $G_{\vec{n}, \mathbb{C}}$ such that the $L$-packet associated to $\varphi_{\vec{n}}$ is $\Pi_{\text {disc }}\left(G_{\vec{n}}(\mathbb{R}), \xi\left(\varphi_{\vec{n}}\right)^{\vee}\right)$. The construction of $\phi_{\mathrm{Ig}, p}^{\vec{n}}$ can be found in [Sh2].

We will need to define a function $f^{\vec{n}}$, which plays the part of a BC-matching function for $\phi_{\operatorname{Ig}}^{\vec{n}}$ for each $\vec{n}$. We already have defined $\left(f^{n}\right)^{\mathfrak{S}}$ and $f_{\mathfrak{S}_{\text {fin }} \backslash\{p\}}^{n}$. We take $\left(f^{n_{1}, n_{2}}\right)^{\mathfrak{S}}=\tilde{\zeta}^{*}\left(\left(f^{n}\right)^{\mathfrak{S}}\right)$ and $f_{\mathfrak{S}_{\text {fin }} \backslash\{p\}}^{n_{1}, n_{2}}=\tilde{\zeta}^{*}\left(f_{\mathfrak{S}_{\text {fin }} \backslash\{p\}}^{n_{1}}\right)$. We also define

$$
f_{\infty}^{\vec{n}}:=e_{\vec{n}}(\Delta) \cdot(-1)^{q(G)}\left\langle\mu_{h}, s_{\vec{n}}\right\rangle \sum_{\varphi_{\vec{n}}} \operatorname{det}\left(\omega_{*}\left(\varphi_{G_{\vec{n}}}\right)\right) \cdot f_{\mathbb{G}_{\vec{n}}, \Xi\left(\varphi_{n}\right)}
$$

where $\varphi_{\vec{n}}$ runs over $L$-parameters such that $\tilde{\eta}_{\vec{n}} \varphi_{\vec{n}} \sim \varphi_{\xi}$ and $\Xi\left(\varphi_{\vec{n}}\right)$ is the algebraic representation of $\mathbb{G}_{\vec{n}}$ arising from $\xi\left(\varphi_{\vec{n}}\right)$. It is straightforward to verify from their definitions that $f_{\infty}^{\vec{n}}$ and $\phi_{\mathrm{Ig}, \infty}^{\vec{n}}$ are BC-matching functions. Finally, we choose $f_{p}^{\vec{n}}$ so that its BC-transfer is $\phi_{\mathrm{Ig}, p}^{\vec{n}}$. (Since $p$ splits in $E$ it can be checked that the base change map defined in section 4.2 of [Sh3] is surjective at p.) We set

$$
f^{\vec{n}}:=\left(f^{\vec{n}}\right)^{\mathfrak{S}} \cdot f_{\mathfrak{S}_{\text {fin }}^{\vec{n}} \backslash\{p\}} \cdot f_{p}^{\vec{n}} \cdot f_{\infty}^{\vec{n}}
$$


The BC-transfer of $f^{\vec{n}}$ coincides with $\phi_{\mathrm{Ig}}^{\vec{n}}$ at places outside $\mathfrak{S}$ (by compatibility of transfers), at $p$ and at $\infty$. At places in $\mathfrak{S}_{\text {fin }} \backslash\{p\}$ we know at least that the BCtransfer of $f^{\vec{n}}$ has the same trace as $\phi_{\mathrm{Ig}}^{\vec{n}}$ against every admissible representation of $G_{\vec{n}}\left(\mathbb{A}_{\mathfrak{S}_{\text {fin }} \backslash\{p\}}\right)$.

By the discussion following Proposition 6.0.6, we can compute

$$
\operatorname{tr}\left(\phi^{\infty, p} \phi_{p}^{\prime} \mid \iota_{l} H_{c}\left(\operatorname{Ig}^{\left(h_{1}, h_{2}\right)}, \mathcal{L}_{\xi}\right)\right)
$$

via the spectral part of the twisted formula, to get

$$
\begin{gathered}
C_{G}(-1)^{h_{1}+h_{2}}\left(\frac{1}{2} \sum_{\Pi^{\prime}} \operatorname{tr}\left(\Pi_{\xi}^{\prime}\left(f^{n}\right) A_{\Pi_{\xi}^{\prime}}^{\prime}\right)+\sum_{\mathbb{G}_{n_{1}, n_{2}, n_{1} \neq n_{2}}} I_{\text {spec }}^{\mathbb{G}_{n_{1}, n_{2}} \theta}\left(f^{n_{1}, n_{2}}\right)\right. \\
+\frac{1}{2} I_{\mathrm{spec}}^{\mathbb{G}_{n} / 2, n / 2} \theta\left(f^{n / 2, n / 2}\right) \\
\left.+\sum_{M \subsetneq \mathbb{G}_{n}} \frac{\left|W_{M}\right|}{\left|W_{\mathbb{G}_{n}}\right|}\left|\operatorname{det}\left(\Phi^{-1} \theta-1\right)_{\mathfrak{a}_{M}}^{\mathbb{G}_{n} \theta}\right|^{-1} \sum_{\Pi_{M}^{\prime}} \operatorname{tr}\left(\mathrm{n}-\operatorname{Ind}_{Q}^{\mathbb{G}_{n}}\left(\Pi_{M}^{\prime}\right)_{\xi}\left(f^{n}\right) \circ A_{\mathrm{n}-\operatorname{Ind}_{Q}^{\prime}\left(\Pi_{M}^{\prime}\right)_{\xi}}^{\prime}\right)\right)
\end{gathered}
$$

where the first sum runs over $\theta$-stable subrepresentations $\Pi^{\prime}$ of $R_{\mathbb{G}_{n}, \text { disc }}$, the sums in the middle run over groups $\mathbb{G}_{n_{1}, n_{2}}$ coming from elliptic endoscopic groups $G_{n_{1}, n_{2}}$ for $G$ (with $n_{1} \geq n_{2}>0$ and some $\left(n_{1}, n_{2}\right)$ possibly excluded). The group $M$ runs over proper Levi subgroups of $\mathbb{G}_{n}$ containing a fixed minimal Levi and $\Pi_{M}^{\prime}$ runs over $\Phi_{n}^{-1} \theta$-stable subrepresentations $\Pi_{M}^{\prime}$ of $R_{M \text {,disc }}$.

We claim that the formula above holds for any $\phi^{\infty, p} \phi_{p}^{\prime}$, without the assumption that it is an acceptable function. To see this, note that Lemma 6.3 of [Sh1] guarantees that there exists some element $f r^{s} \in J^{\left(h_{1}, h_{2}\right)}\left(\mathbb{Q}_{p}\right)$ such that $\phi^{\infty, p}\left(\phi_{p}^{\prime}\right)^{(N)}(g)=\phi^{\infty, p}(g) \phi_{p}^{\prime}\left(g\left(f r^{s}\right)^{N}\right)$ is acceptable for any sufficiently large $N$. (The paper [Sh1] treats general Igusa varieties, and it is easy to check that our case is covered.) So the equality of (6.5) and (6.6) holds when $\phi_{p}^{\prime}$ is replaced by 
$\left(\phi_{p}^{\prime}\right)^{(N)}$. Both (6.5) and (6.6) are finite linear combinations of terms of the form $\operatorname{tr} \rho\left(\left(\phi_{p}^{\prime}\right)^{(N)}\right)$ where $\rho \in \operatorname{Irr}\left(J^{\left(h_{1}, h_{2}\right)}\left(\mathbb{Q}_{p}\right)\right)$. In order to see that this is true for (6.6), we need to translate it from computing the trace of $f^{\vec{n}}$ to computing the trace of $\phi_{\mathrm{Ig}}^{\vec{n}}$ to computing the trace of $\phi_{p}^{\prime}$, using Lemmas 6.0.8 and 6.0.9. Now the same argument as that for Lemma 6.4 of [Sh1] shows that (6.5) and (6.6) are equal for $\phi^{\infty, p}\left(\phi_{p}^{\prime}\right)^{(N)}$ for every integer $N$, in particular for $N=0$. Thus, we can work with arbitrary $\phi^{\infty, p} \phi_{p}^{\prime}$.

Choose a decomposition of the normalized intertwining operators

$$
A_{\Pi^{1}}^{\prime}=A_{\Pi^{1, \mathfrak{G}}}^{\prime} A_{\Pi_{\mathfrak{G}_{\mathrm{fin}}}^{1}}^{\prime} A_{\Pi_{\infty}^{1}}^{\prime}
$$

Set

$$
\frac{A_{\Pi^{1}}^{\prime}}{A_{\Pi^{1}}^{0}}:=\frac{A_{\Pi^{1, \mathfrak{S}}}^{\prime}}{A_{\Pi^{1, \mathfrak{S}}}^{0}} \cdot \frac{A_{\Pi_{\mathfrak{S}_{\text {fin }}}^{1}}^{\prime}}{A_{\Pi_{\mathfrak{S}_{\text {fin }}}^{1}}^{0}} \cdot \frac{A_{\Pi_{\infty}^{1}}^{\prime}}{A_{\Pi_{\infty}^{1}}^{0}} \in\{ \pm 1\},
$$

where the denominators on the right side are the normalized interwiners chosen above. In the sum (6.6), the third term evaluates the trace of $f^{n}$ against representations induced from proper Levi subgroups. The second term has a similar form: outside the set $\mathfrak{S}$ we have the identity $\left(f^{n_{1}, n_{2}}\right)^{\mathfrak{S}}=\tilde{\zeta}^{*}\left(\left(f^{n}\right)^{\mathfrak{S}}\right)$ and formula 4.17 of [Sh3] tells us that

$$
\operatorname{tr} \Pi_{M}^{\mathfrak{S}}\left(\tilde{\zeta}_{n_{1}, n_{2}}^{*}\left(f^{n}\right)^{\mathfrak{S}}\right)=\operatorname{tr}\left(\tilde{\zeta}_{n_{1}, n_{2} *}\left(\Pi_{M}^{\mathfrak{S}}\right)\right)\left(f^{n}\right)^{\mathfrak{S}}
$$

where $\tilde{\zeta}_{n_{1}, n_{2} *}$ is the transfer from $\mathbb{G}_{n_{1}, n_{1}}$ to $\mathbb{G}_{n}$ on the representation-theoretic side and consists of taking the parabolic induction of a twist of $\Pi_{M}^{\mathfrak{S}}$. The multiplicity one result of Jacquet and Shalika (see page 200 of [AC]) implies that the string of Satake parameters outside a finite set $\mathfrak{S}$ of a cuspidal automorphic representation of $G L_{n}\left(\mathbb{A}_{F}\right)$ unramified outside $\mathfrak{S}$ cannot coincide with the string of Satake parameters outside $\mathfrak{S}$ of an automorphic representation of $G L_{n}\left(\mathbb{A}_{F}\right)$ which is a subquotient of a representation induced from a proper Levi subgroup. 
Thus, if we are interested in the $\Pi^{1, \mathfrak{S}_{-}}$-part of $\operatorname{tr}\left(\phi^{\infty, p} \phi_{p}^{\prime} \mid \iota_{l} H_{c}\left(\operatorname{Ig}^{\left(h_{1}, h_{2}\right)}, \mathcal{L}_{\xi}\right)\right)$, then only the first term of (6.6) can contribute to it.

Thus, we are left to consider

$$
\begin{gathered}
C_{G}(-1)^{h_{1}+h_{2}}\left(\frac{1}{2} \frac{A_{\Pi^{1}}^{\prime}}{A_{\Pi^{1}}^{0}} \chi_{\Pi^{1, \mathfrak{S}}}\left(\left(f^{n}\right)^{\mathfrak{S}}\right) \operatorname{tr}\left(\Pi_{\mathfrak{S}}^{1}\left(f_{\mathfrak{S}}^{n}\right) A_{\Pi_{\mathfrak{S}}^{1}}^{0}\right)\right. \\
\left.+\sum_{\left(\Pi^{\prime}\right)^{\mathfrak{S} \neq \Pi^{1, \mathfrak{S}}}} \chi_{\left(\Pi^{\prime}\right) \mathfrak{S}}\left(\left(f^{n}\right)^{\mathfrak{S}}\right) \times\left(\begin{array}{c}
\text { expression in } \\
\text { terms of } f_{\mathfrak{S}}^{n}
\end{array}\right)\right)
\end{gathered}
$$

where $\left(\Pi^{\prime}\right)^{\mathfrak{S}}$ runs over a set of unramified representations of $\mathbb{G}_{n}\left(\mathbb{A}^{\mathfrak{S}}\right)$. On the other hand, we can also decompose $\operatorname{tr}\left(\phi^{\infty, p} \phi_{p}^{\prime} \mid \iota_{l} H_{c}\left(\operatorname{Ig}^{\left(h_{1}, h_{2}\right)}, \mathcal{L}_{\xi}\right)\right)$ into a $\Pi^{1, \mathfrak{S}_{-}}$ part and $\left(\pi^{\prime}\right)^{\mathfrak{S}}$-parts, where $B C\left(\left(\pi^{\prime}\right)^{\mathfrak{S}}\right) \neq \Pi^{1, \mathfrak{S}}$. We conclude as in [Sh3] that

$\operatorname{tr}\left(\phi_{\mathfrak{S}_{\text {fin }} \backslash\{p\}} \phi_{p}^{\prime} \mid \iota_{l} H_{c}\left(\operatorname{Ig}^{\left(h_{1}, h_{2}\right)}, \mathcal{L}_{\xi}\right)\left\{\Pi^{1, \mathfrak{S}}\right\}\right)=(-1)^{h_{1}+h_{2}} \frac{C_{G}}{2} \frac{A_{\Pi^{1}}^{\prime}}{A_{\Pi^{1}}^{0}} \cdot \operatorname{tr}\left(\Pi_{\mathfrak{S}}\left(f_{\mathfrak{S}}^{n}\right) A_{\Pi_{\mathfrak{S}}^{0}}\right)$.

Now $\Pi_{\infty}^{1} \simeq \Pi_{\Xi}$, so $\operatorname{tr}\left(\Pi_{\infty}^{1}\left(f_{\infty}^{n}\right) A_{\Pi_{\infty}}^{0}\right)=2(-1)^{q(G)}=2$. We also have

$$
\operatorname{tr}\left(\Pi_{p}^{1}\left(f_{p}^{n}\right) A_{\Pi_{p}}^{0}\right)=\operatorname{tr} \iota_{l} \pi_{p}\left(\phi_{\mathrm{Ig}, p}^{n}\right)=\operatorname{tr} \iota_{l} \operatorname{Red}_{n}^{\left(h_{1}, h_{2}\right)}\left(\pi_{p}\right)\left(\phi_{p}^{\prime}\right)
$$

by Lemma 6.0 .8 and

$$
\operatorname{tr}\left(\Pi_{\mathfrak{S}_{\mathrm{fin}} \backslash\{p\}}^{1}\left(f_{\mathfrak{S}_{\mathrm{fin}} \backslash\{p\}}^{n}\right) A_{\Pi_{\mathfrak{S}_{\mathrm{fin}} \backslash\{p\}}^{1}}^{0}\right)=\operatorname{tr} \iota_{l} \pi_{p}\left(\phi_{\mathfrak{S}_{\mathrm{fin}} \backslash\{p\}}\right) .
$$

Putting together (6.7), (6.8) and (6.9) and applying $B C_{\mathfrak{S}_{\text {fin } \backslash\{p\}}}$ we get the desired result with $e_{0}=A_{\Pi^{1}}^{\prime} / A_{\Pi^{1}}^{0}$ which is independent of $\left(h_{1}, h_{2}\right)$. 


\section{Chapter 7}

\section{Proof of the main theorem}

Let $E / \mathbb{Q}$ be an imaginary quadratic field in which $p$ splits. Let $F_{1} / \mathbb{Q}$ be a totally real field and let $w$ be a prime of $F_{1}$ above $p$. Set $F^{\prime}=E F_{1}$. Let $F_{2}$ be a totally real quadratic extension of $\mathbb{Q}$, in which $w=w_{1} w_{2}$ splits and set $F=E F_{2}$. Let $n \in \mathbb{Z}_{\geq 2}$. Also denote $F_{2}$ by $F^{+}$. Let $\Pi$ be a cuspidal automorphic representation of $G L_{n}\left(\mathbb{A}_{F^{\prime}}\right)$.

Consider the following assumptions on $\left(E, F^{\prime}, F, \Pi\right)$ :

- $\left[F_{1}: \mathbb{Q}\right] \geq 2$;

- $\operatorname{Ram}_{F / \mathbb{Q}} \cup \operatorname{Ram}_{\mathbb{Q}}(\varpi) \cup \operatorname{Ram}_{\mathbb{Q}}(\Pi) \subset \operatorname{Spl}_{F / F^{+}, \mathbb{Q}}$

- $(\Pi)^{\vee} \simeq \Pi \circ c ;$

- $\Pi_{\infty}$ is cohomological for an irreducible algebraic representation $\Xi$ of $G L_{n}\left(F^{\prime} \otimes_{\mathbb{Q}}\right.$ C).

- $B C_{F / F^{\prime}}(\Pi)$ is cuspidal

Set $\Pi^{0}=B C_{F / F^{\prime}}(\Pi)$ and $\Xi^{0}=B C_{F / F^{\prime}}(\Xi)$. The following lemma is the same as Lemma 7.2 of [Sh3]. 
Lemma 7.0.11. Let $\Pi^{0}$ and $\Xi^{0}$ as above. We can find a character $\psi: \mathbb{A}_{E}^{\times} / E^{\times} \rightarrow$ $\mathbb{C}^{\times}$and an algebraic representation $\xi_{\mathbb{C}}$ of $G$ over $\mathbb{C}$ satisfying the following conditions

- $\psi_{\Pi^{0}}=\psi^{c} / \psi$

- $\Xi^{0}$ is isomorphic to the restriction of $\Xi^{\prime}$ to $R_{F / \mathbb{Q}}\left(G L_{n}\right) \times_{\mathbb{Q}} \mathbb{C}$, where $\Xi^{\prime}$ is obtained from $\xi_{\mathbb{C}}$ by base change from $G$ to $\mathbb{G}_{n}$;

- $\left.\xi_{\mathbb{C}}\right|_{E_{\infty}^{\times}} ^{-1}=\psi_{\infty}^{x}$, and

- $\operatorname{Ram}_{\mathbb{Q}}(\psi) \subset \operatorname{Spl}_{F / F^{+}, \mathbb{Q}}$.

Moreover, if l splits in $E$ then

- $\psi_{\mathcal{O}_{E_{u}}^{\times}}=1$ where $u$ is the place above l induced by $\left.\iota_{l}^{-1} \tau\right|_{E}$.

Set $\Pi^{1}=\psi \otimes \Pi^{0}$. Then $\Pi^{1}$ is a cuspidal automorphic representation of $G L_{1}\left(\mathbb{A}_{E}\right) \times G L_{n}\left(\mathbb{A}_{F}\right)$. Let $\xi=\iota_{l} \xi_{\mathbb{C}}$, where $\xi_{\mathbb{C}}$ is as in Lemma 7.0.11.

Let $\mathcal{A}_{U}$ be the universal abelian variety over $X_{U}$. Since $\mathcal{A}_{U}$ is smooth over $X_{U}, \mathcal{A}_{U}^{m_{\xi}}$ satisfies the conditions in Section 4.3. In particular, $\mathcal{A}_{U}^{m_{\xi}}$ is locally etale over a product of strictly semistable schemes. For $S, T \subseteq\{1, \ldots, n\}$, let $\mathcal{A}_{U, S, T}^{m_{\xi}}=\mathcal{A}_{U}^{m_{\xi}} \times_{X_{U}} Y_{U, S, T}$.

Define the following admissible $G\left(\mathbb{A}^{\infty, p}\right)$-modules with a commuting continuous action of $\operatorname{Gal}\left(\bar{F}^{\prime} / F^{\prime}\right)$ :

$$
\begin{gathered}
H^{j}\left(X_{\mathrm{Iw}(m)}, \mathcal{L}_{\xi}\right)=\lim _{\overrightarrow{U^{p}}} H^{j}\left(X_{U} \times F^{\prime} \bar{F}^{\prime}, \mathcal{L}_{\xi}\right)=H^{j}\left(X, \mathcal{L}_{\xi}\right)^{\mathrm{Iw}(m)}, \\
H^{j}\left(\mathcal{A}_{\mathrm{Iw}(m)}^{m_{\xi}}, \overline{\mathbb{Q}}_{l}\right)=\lim _{\overrightarrow{U^{p}}} H^{j}\left(\mathcal{A}_{U}^{m_{\xi}} \times_{F^{\prime}} \bar{F}^{\prime}, \overline{\mathbb{Q}}_{l}\right) .
\end{gathered}
$$

Also define the admissible $G\left(\mathbb{A}^{\infty, p}\right) \times\left(\text { Frob }_{\mathbb{F}}\right)^{\mathbb{Z}}$-module

$$
H^{j}\left(\mathcal{A}_{\mathrm{Iw}(m), S, T}^{m_{\xi}}, \overline{\mathbb{Q}}_{l}\right)=\lim _{\overrightarrow{U^{p}}} H^{j}\left(\mathcal{A}_{U, S, T}^{m_{\xi}} \times_{\mathbb{F}} \overline{\mathbb{F}}, \overline{\mathbb{Q}}_{l}\right) .
$$


Note that $a_{\xi}$ is an idempotent on $H^{j}\left(\mathcal{A}_{\mathrm{Iw}(m), S, T}^{m_{\xi}}, \overline{\mathbb{Q}}_{l}\left(t_{\xi}\right)\right)$ and

$$
a_{\xi} H^{j+m_{\xi}}\left(\mathcal{A}_{\mathrm{Iw}(m), S, T}^{m_{\xi}}, \overline{\mathbb{Q}}_{l}\left(t_{\xi}\right)\right)=H^{j}\left(Y_{\mathrm{Iw}(m), S, T}, \mathcal{L}_{\xi}\right)
$$

Proposition 7.0.12. For each rational prime $l \neq p$ there is a $G\left(\mathbb{A}^{\infty, p}\right) \times$ $\left(\text { Frob }_{\mathbb{F}}\right)^{\mathbb{Z}}$-equivariant spectral sequence with a nilpotent operator $N$

$$
\begin{gathered}
B C^{p}\left(E_{1}^{i, m+m_{\xi}-i}(\operatorname{Iw}(m), \xi)\left[\Pi^{1, \mathfrak{S}}\right]\right) \Rightarrow \\
B C^{p}\left(W D\left(\left.H^{m}\left(X_{\operatorname{Iw}(m)}, \mathcal{L}_{\xi}\right)\right|_{\operatorname{Gal}(\bar{K} / K)}\left[\Pi^{1, \mathfrak{S}}\right]\right)^{F-s s}\right),
\end{gathered}
$$

where

$$
\begin{gathered}
B C^{p}\left(E_{1}^{i, m+m_{\xi}-i}(\operatorname{Iw}(m), \xi)\left[\Pi^{1, \mathfrak{S}}\right]\right)= \\
\bigoplus_{k-l=-i} B C^{p}\left(a_{\xi} H^{m+m_{\xi}}\left(\mathcal{A}_{\mathrm{Iw}(m)}^{m_{\xi}}, \operatorname{Gr}^{l} \operatorname{Gr}_{k} R \psi \overline{\mathbb{Q}}_{l}\left(t_{\xi}\right)\right)\left[\Pi^{1, \mathfrak{S}}\right]\right) .
\end{gathered}
$$

The action of $N$ sends $B C^{p}\left(a_{\xi} H^{m+m_{\xi}}\left(\mathcal{A}_{\mathrm{Iw}(m)}^{m_{\xi}}, \operatorname{Gr}^{l} \operatorname{Gr}_{k} R \psi \overline{\mathbb{Q}}_{l}\left(t_{\xi}\right)\right)\left[\Pi^{1, \mathfrak{S}}\right]\right)$ to

$$
B C^{p}\left(a_{\xi} H^{m+m_{\xi}}\left(\mathcal{A}_{\mathrm{Iw}(m)}^{m_{\xi}}, \operatorname{Gr}^{l+1} \operatorname{Gr}_{k-1} R \psi \overline{\mathbb{Q}}_{l}\left(t_{\xi}\right)\right)\left[\Pi^{1, \mathfrak{S}}\right]\right) .
$$

Furthermore, there is a direct sum decomposition

$B C^{p}\left(a_{\xi} H^{m+m_{\xi}}\left(\mathcal{A}_{\mathrm{Iw}(m)}^{m_{\xi}}, \operatorname{Gr}^{l} \operatorname{Gr}_{k} R \psi \overline{\mathbb{Q}}_{l}\left(t_{\xi}\right)\right)\left[\Pi^{1, \mathfrak{S}}\right]\right) \simeq \bigoplus_{j \geq 0} B C^{p}\left(M_{j, m+m_{\xi}-j}(k, l)\right)$,

where

$$
B C^{p}\left(M_{j, m+m_{\xi}-j}(k, l)\right)=\bigoplus_{s=1}^{k+l} \bigoplus_{\# S=j+s, \# T=j+k+l-s+1} H_{S, T}^{j+m_{\xi}, s}(k, l)
$$


and

$$
\begin{gathered}
H_{S, T}^{j+m_{\xi}, s}(k, l)=B C^{p}\left(a_{\xi} H^{m+m_{\xi}-2 j-k-l+1}\left(\mathcal{A}_{\mathrm{Iw}(m), S, T}^{m_{\xi}}, \overline{\mathbb{Q}}_{l}\left(t_{\xi}-j-k+1\right)\right)\left[\Pi^{1, \mathfrak{S}}\right]\right) \\
=B C^{p}\left(H^{m-2 j-k-l+1}\left(Y_{\mathrm{Iw}(m), S, T}, \overline{\mathbb{Q}}_{l}(-j-k+1)\right)\left[\Pi^{1, \mathfrak{S}}\right]\right) .
\end{gathered}
$$

Proof. Note that $\mathcal{A}_{U}^{m_{\xi}} / \mathcal{O}_{K}$ satisfies the hypotheses of Section 4. We have a spectral sequence of $G\left(\mathbb{A}^{\infty, p}\right) \times\left(\text { Frob }_{\mathbb{F}}\right)^{\mathbb{Z}}$-modules with a nilpotent operator $N$ :

$$
E_{1}^{i, m-i}(\operatorname{Iw}(m), \xi) \Rightarrow H^{m}\left(\mathcal{A}_{U}^{m_{\xi}} \times_{F^{\prime}} \bar{F}_{\mathfrak{p}}^{\prime}, \overline{\mathbb{Q}}_{l}(t)\right)
$$

where

$$
E_{1}^{i, m-i}(\operatorname{Iw}(m), \xi)=\bigoplus_{k-l=-i} H^{m}\left(\mathcal{A}_{U}^{m_{\xi}} \times_{\mathbb{F}} \overline{\mathbb{F}}, G r^{l} G r_{k} R \psi \overline{\mathbb{Q}}_{l}(t)\right)
$$

$N$ will send $H^{m}\left(\mathcal{A}_{U}^{m_{\xi}}, \operatorname{Gr}^{l} \operatorname{Gr}_{k} R \psi \overline{\mathbb{Q}}_{l}(t)\right)$ to $H^{m}\left(\mathcal{A}_{U}^{m_{\xi}}, \operatorname{Gr}^{l+1} \operatorname{Gr}_{k-1} R \psi \overline{\mathbb{Q}}_{l}(t)\right)$.

By Corollary 4.3.10, we also have a $G\left(\mathbb{A}^{\infty, p}\right) \times\left(\text { Frob }_{\mathbb{F}}\right)^{\mathbb{Z}}$-equivariant isomorphism

$$
H^{m}\left(\mathcal{A}_{U}^{m_{\xi}} \times_{\mathbb{F}} \overline{\mathbb{F}}, G r^{l} G r_{k} R \psi \overline{\mathbb{Q}}_{l}(t)\right) \simeq \bigoplus_{j \geq 0} M_{j, m-j}(k, l)
$$

where

$$
M_{j, m-j}(k, l)=\bigoplus_{s=1}^{k+l} \bigoplus_{\substack{\# S=j+s \\ \# T=j+k+l-s+1}} H_{S, T}^{j, s}(k, l)
$$

and

$$
H_{S, T}^{j, s}(k, l)=H^{m-2 j-k-l+1}\left(\mathcal{A}_{U, S, T}^{m_{\xi}} \times_{\mathbb{F}} \overline{\mathbb{F}}, \overline{\mathbb{Q}}_{l}(t-j-k+1)\right) .
$$

We take $t=t_{\xi}$, apply $a_{\xi}$, replace $j$ by $j+m_{\xi}$ and take the inverse limit over $U^{p}$. We get a spectral sequence of $G\left(\mathbb{A}^{\infty, p}\right) \times\left(\text { Frob }_{\mathbb{F}}\right)^{\mathbb{Z}}$-modules, converging to $H^{j}\left(X_{\mathrm{Iw}(m)}, \mathcal{L}_{\xi}\right)$. We identify $H^{j}\left(X_{\mathrm{Iw}(m)}, \mathcal{L}_{\xi}\right)$ with its associated Weil-Deligne representation and we semisimplify the action of Frobenius. After taking $\Pi^{1, \mathfrak{S}_{-}}$ 
isotypical components and applying $B C^{p}$ we get the desired spectral sequence.

Corollary 7.0.13. Keep the assumptions made in the beginning of this section.

The Weil-Deligne representation

$$
W D\left(B C^{p}\left(\left.H^{2 n-2}\left(X_{\operatorname{Iw}(m)}, \mathcal{L}_{\xi}\right)\right|_{\operatorname{Gal}(\bar{K} / K)}\left[\Pi^{1, \mathfrak{S}}\right]\right)\right)^{F-s s}
$$

is pure of weight $m_{\xi}-2 t_{\xi}+2 n-2$.

Proof. By Proposition 5.3.5,

$$
B C^{p}\left(H^{j}\left(Y_{\operatorname{Iw}(m), S, T}, \mathcal{L}_{\xi}\right)\left[\Pi^{1, \mathfrak{S}}\right]\right)=0
$$

unless $j=2 n-\# S-\# T$. Thus, the terms of the direct sum decomposition

$$
B C^{p}\left(M_{j, m+m_{\xi}-j}(k, l)\right)
$$

which are all of the form

$$
B C^{p}\left(H^{m-2 j-k-l+1}\left(Y_{\mathrm{Iw}(m), S, T}, \overline{\mathbb{Q}}_{l}(-j-k+1)\right)\left[\Pi^{1, \mathfrak{S}}\right]\right)
$$

with $\# S=j+s$ and $\# T=j+k+l-s+1$, vanish unless $m=2 n-2$. This means that the terms of the spectral sequence $B C^{p}\left(E_{1}^{i, m+m_{\xi}-i}(\operatorname{Iw}(m), \xi)\left[\Pi^{1, \mathfrak{S}}\right]\right)$ vanish unless $m=2 n-2$. If $m=2 n-2$ then each summand of

$$
B C^{p}\left(E_{1}^{i, 2 n-2+m_{\xi}-i}(\operatorname{Iw}(m), \xi)\left[\Pi^{1, \mathfrak{S}}\right]\right)
$$

has a filtration with graded pieces

$$
B C^{p}\left(H^{2 n-2-2 j-k-l+1}\left(Y_{\mathrm{Iw}(m), S, T}, \mathcal{L}_{\xi}(-j-k+1)\right)\left[\Pi^{1, \mathfrak{S}}\right]\right),
$$


where $k-l=-i$. These graded pieces are strictly pure of weight $m_{\xi}-2 t_{\xi}+$ $2 n-2+k-l-1$, which only depends on $i$. Thus, the whole of

$$
B C^{p}\left(E_{1}^{i, 2 n-2+m_{\xi}-i}(\operatorname{Iw}(m), \xi)\left[\Pi^{1, \mathfrak{S}}\right]\right)
$$

is strictly pure of weight $m_{\xi}-2 t_{\xi}+2 n-2-i-1$. The spectral sequence degenerates at $E_{1}$, since $E_{1}^{i, m-i}=0$ unless $m=2 n-2$ and also the abutment is pure of weight $m_{\xi}-2 t_{\xi}+2 n-2$. Thus,

$$
B C^{p}\left(W D\left(\left.H^{m}\left(X_{\operatorname{Iw}(m)}, \mathcal{L}_{\xi}\right)\right|_{\operatorname{Gal}_{(\bar{K} / K)}}\left[\Pi^{1, \mathfrak{S}}\right]\right)^{F-s s}\right)
$$

vanishes for $m \neq 2 n-2$ and is pure of weight $m_{\xi}-2 t_{\xi}+2 n-2$ for $m=2 n-2$.

Theorem 7.0.14. Let $n \in \mathbb{Z}_{\geq 2}$ be an integer and $L$ be any $C M$ field. Let $l$ be a prime and $\iota_{l}$ be an isomorphism $\iota_{l}: \overline{\mathbb{Q}}_{l} \rightarrow \mathbb{C}$. Let $\Pi$ be a cuspidal automorphic representation of $G L_{n}\left(\mathbb{A}_{L}\right)$ satisfying

- $\Pi^{\vee} \simeq \Pi \circ c$

- $\Pi$ is cohomological for some irreducible algebraic representation $\Xi$.

Let

$$
R_{l}(\Pi): \operatorname{Gal}(\bar{L} / L) \rightarrow G L_{n}\left(\overline{\mathbb{Q}}_{l}\right)
$$

be the Galois representation associated to $\Pi$ by [Sh3, CH]. Let $p \neq l$ and let $y$ be a place of $L$ above $p$. Then we have the following isomorphism of Weil-Deligne respresentations

$$
W D\left(\left.R_{l}(\Pi)\right|_{\operatorname{Gal}\left(\bar{L}_{y} / L_{y}\right)}\right)^{F-s s} \simeq \iota_{l}^{-1} \mathcal{L}_{n, L_{y}}\left(\Pi_{y}\right)
$$

Proof. This theorem has been proven by [Sh3] except in the case when $n$ is even and $\Xi$ is not slightly regular. In that exceptional case it is still known that 
we have an isomorphism of semisimplified $W_{L_{y}}$-representations by $[\mathrm{CH}]$, so it remains to check that the two monodromy operators $N$ match up. By Corollary 5.3.4, $\Pi_{y}$ is tempered. This is equivalent to $\iota_{l}^{-1} \mathcal{L}_{n, L_{y}}\left(\Pi_{y}\right)$ being pure of weight $2 n-2$. In order to get an isomorphism of Weil-Deligne representations, it suffices to prove that $W D\left(\left.R_{l}(\Pi)\right|_{\operatorname{Gal}\left(\bar{L}_{y} / L_{y}\right)}\right)^{F-s s}$ is pure.

We first will find a CM field $F^{\prime}$ such that

- $F^{\prime}=E F_{1}$, where $E$ is an imaginary quadratic field in which $p$ splits and $F_{1}=\left(F^{\prime}\right)^{c=1}$ has $\left[F_{1}: \mathbb{Q}\right] \geq 2$,

- $F^{\prime}$ is soluble and Galois over $L$,

- $\Pi_{F^{\prime}}^{0}=B C_{F^{\prime} / L}(\Pi)$ is a cuspidal automorphic representation of $G L_{n}\left(\mathbb{A}_{F^{\prime}}\right)$, and

- there is a place $\mathfrak{p}$ of $F$ above $y$ such that $\Pi_{F^{\prime}, \mathfrak{p}}^{0}$ has an Iwahori fixed vector, and a CM field $F$ which is a quadratic extension of $F^{\prime}$ such that

- $\mathfrak{p}=\mathfrak{p}_{1} \mathfrak{p}_{2}$ splits in $F$,

- $\operatorname{Ram}_{F / \mathbb{Q}} \cup \operatorname{Ram}_{\mathbb{Q}}(\varpi) \cup \operatorname{Ram}_{\mathbb{Q}}(\Pi) \subset \operatorname{Spl}_{F / F^{\prime}, \mathbb{Q}}$, and

- $\Pi_{F}^{0}=B C_{F / F^{\prime}}\left(\Pi_{F^{\prime}}^{0}\right)$ is a cuspidal automorphic representation of $G L_{n}\left(\mathbb{A}_{F}\right)$.

To find $F$ and $F^{\prime}$ we proceed as in the proof of Corollary 5.3.4. Set $\Pi_{F}^{1}=\Pi_{F}^{0} \otimes \psi$, where $\psi$ is chosen as in Lemma 7.0.11.

We claim that we have isomorphisms

$$
C_{G} \cdot\left(\left.R_{l}(\Pi)\right|_{G a l\left(\bar{F}^{\prime} / F^{\prime}\right)}\right)^{\otimes 2} \simeq C_{G} \cdot R_{l}\left(\Pi_{F^{\prime}}^{0}\right)^{\otimes 2} \simeq \tilde{R}_{l}^{2 n-2}\left(\Pi_{F}^{1}\right) \otimes R_{l}(\psi)^{-1},
$$

where $\tilde{R}_{l}^{k}\left(\Pi_{F}^{1}\right)$ was defined in Section 4. The first isomorphism is clear. The second isomorphism can be checked by Chebotarev locally at unramified places, 
using the local global compatibility for $R_{l}\left(\Pi_{F^{\prime}}^{0}\right)$ and the formula

$$
\tilde{R}_{l}\left(\Pi_{F}^{1}\right)=e_{0} C_{G} \cdot\left[\left.\left(\pi_{p, 0} \circ \operatorname{Art}_{\mathbb{Q}_{p}}^{-1}\right)\right|_{W_{F_{\mathfrak{p}}^{\prime}}} \otimes \iota_{l}^{-1} \mathcal{L}_{F_{\mathfrak{p}}^{\prime}, n}\left(\Pi_{F^{\prime}, \mathfrak{p}}^{0}\right)^{\otimes 2}\right]
$$

(It can be checked easily, either by computing the weight or by using the spectral sequences above that $\tilde{R}_{l}^{k}\left(\Pi_{F}^{1}\right) \neq 0$ if and only if $k=2 n-2$ and thus that $e_{0}=(-1)^{2 n-2}=1$.)

We also have

$$
B C^{p}\left(H^{2 n-2}\left(X_{\mathrm{Iw}(m)}, \mathcal{L}_{\xi}\right)\left[\Pi_{F}^{1, \mathfrak{S}}\right]\right) \simeq\left(\operatorname{dim} \pi_{p}^{\mathrm{Iw}(m)}\right) \cdot \iota_{l}^{-1} \Pi^{\infty, p} \otimes \tilde{R}^{2 n-2}\left(\Pi_{F}^{1}\right)
$$

as admissible representations of $G\left(\mathbb{A}^{\infty, p}\right) \times \operatorname{Gal}\left(\bar{F}^{\prime} / F^{\prime}\right)$. By Corollary 7.0.13, $W D\left(\left.\tilde{R}_{l}^{2 n-2}\left(\Pi_{F}^{1}\right)\right|_{G a l\left(\bar{F}_{\mathfrak{p}}^{\prime} / F_{\mathfrak{p}}^{\prime}\right)}\right)$ is pure of weight $m_{\xi}-2 t_{\xi}+2 n-2$. By Lemma 1.7 of $[\mathrm{TY}]$,

$$
W D\left(\left.R_{l}\left(\Pi_{F^{\prime}}^{0}\right)^{\otimes 2}\right|_{\operatorname{Gal}\left(\bar{F}_{\mathfrak{p}}^{\prime} / F_{\mathfrak{p}}^{\prime}\right)}\right)
$$

is also pure. It has weight $2 n-2$. The monodromy operator acts on $\left.R_{l}\left(\Pi_{F^{\prime}}^{0}\right)^{\otimes 2}\right|_{W_{F_{\mathfrak{p}}^{\prime}}}$ as $1 \otimes N+N \otimes 1$, where $N$ is the monodromy operator on $\left.R_{l}\left(\Pi_{F^{\prime}}^{0}\right)\right|_{W_{F_{\mathfrak{p}}^{\prime}}}$. We wish to show that $V:=W D\left(\left.R_{l}\left(\Pi_{F^{\prime}}^{0}\right)\right|_{W_{F_{\mathfrak{p}}^{\prime}}}\right)^{F-s s}$ is pure of weight $n-1$. Consider the direct sum decomposition $V=\oplus_{i \in \mathbb{Z}} V_{i}$, where $V_{i}$ is strictly pure of weight $n-1+i$. It suffices to prove that $N^{i}: V_{i} \rightarrow V_{-i}$ is injective for every $i>0$, since then we can compare dimensions to deduce that $N^{i}$ is an isomorphism. Let $x \in V_{i}$ and assume that $N^{i} x=0$. Since $x \in V_{i}$, the vector $x \otimes x$ belongs to the subspace of $W D\left(\left.R_{l}\left(\Pi_{F^{\prime}}^{0}\right)^{\otimes 2}\right|_{W_{F_{\mathfrak{p}}^{\prime}}}\right)^{F-s s}$ which is strictly pure of weight $2 n-2+2 i$. But then

$$
N^{2 i}(x \otimes x)=\sum_{k=0}^{2 i} N^{k} x \otimes N^{2 i-k} x=0,
$$

which contradicts the purity of $W D\left(\left.R_{l}\left(\Pi_{F^{\prime}}^{0}\right) \otimes 2\right|_{W_{F_{p}^{\prime}}}\right)^{(F-s s)}$. Thus, $W D\left(\left.R_{l}\left(\Pi_{F^{\prime}}^{0}\right)\right|_{G a l\left(\bar{F}_{\mathfrak{p}}^{\prime} / F_{\mathfrak{p}}^{\prime}\right)}\right)^{F-s s}$ 
has to be pure. By Lemma 1.4 of [TY], purity is preserved under finite extensions, so $W D\left(\left.R_{l}(\Pi)\right|_{\operatorname{Gal}\left(\bar{L}_{y} / L_{y}\right)}\right)^{F-s s}$ is also pure. 


\section{Bibliography}

[AC] Arthur, J. and Clozel, L.: Simple algebras, base change, and the advanced theory of the trace formula, Princeton University Press, no. 120, Ann. of Math. Studies, Princeton, New Jersey (1989).

[Art] Arthur, J.: The invariant trace formula II, Global theory, J. Amer. Math. Soc. 1 (1988), 501-554.

[Bad] Badulescu, A.I.: Jacquet-Langlands et unitarisabilite, J. Inst. Math. Jussieu 6 (2007), 349-379.

[BLGGT1] Barnet-Lamb, T., Gee, T., Geraghty, D. and Taylor, R: Local-global compatibility for $l=p$, I, preprint (2011).

[BLGGT2] Barnet-Lamb, T., Gee, T., Geraghty, D. and Taylor, R: Local-global compatibility for $l=p$, II, preprint (2011).

[Be] Beilinson, A.A.: On the derived category of perverse sheaves, in $K$-theory, arithmetic and geometry (Moscow, 1984-1986) Lecture Notes in Math., 1289, Springer Berlin, (1987), 27-41.

[BBD] Beilinson, A.A., Bernstein, J. and Deligne, P.: Faisceaux pervers, Asterisque 100, SMF (1982). 
[BZ] Bernstein, I.N and Zelevinsky, A.V.: Induced representations of reductive p-adic groups I, Ann. Sci. ENS (4) 10 (1997), 441-472.

[Bor] Borel, A.: Automorphic $L$-functions, pp. 27-61 in Borel and Casselman (eds.): Automorphic forms, representations and $L$-functions, Proc. of Symp. in Pure Math., vol. 33.2, Providence, RI, AMS, Amer. Math. Soc., 1979.

[Car] Caraiani, A.: Local-global compatibility and monodromy for $l=p$, preprint, arXiv:1202.4683.

[CH] Chenevier, G. and Harris, M.: Construction of automorphic Galois representations, II, http://people.math.jussieu.fr/ harris/ConstructionII.pdf.

[Cl1] Clozel, L.: Representations galoisiennes associes aux representations automorphes autoduales de $G L(n)$, Publ. Math. I.H.E.S. 73 (1991), 97-145.

[Cl2] Clozel, L.: Purity reigns supreme, http://fa.institut.math.jussieu.fr/node/45.

[De1] Deligne, P.: La conjecture de Weil I, Inst. Hautes Etudes Sci. Publ. Math. 43 (1974), 273-307.

[De2] Deligne, P.: La conjecture de Weil II, Inst. Hautes Etudes Sci. Publ. Math. 52 (1980), 137-252.

[D] Drinfeld, V. G.: Elliptic modules, Math. USSR Sbornik 23 (1974), no. 4, 561-592.

[EGA3] Grothendieck, A. and Dieudonne, J.: Elements de geometrie algebrique III. Etude cohomologique des faisceaux coherents, Seconde partie, Publ. Math. I.H.E.S. 17 (1963), 5-91.

[EGA4] Grothendieck, A and Dieudonne, J.: Elements de geometrie algebrique IV. Etude locale des schemas et des morphismes de schemas, Quatrieme partie, Publ. Math. I.H.E.S. 32 (1967), 5-361. 
[E] Ekedahl, T.: On the adic formalism, in The Grothendieck Festschrift Volume II, Progress in Math, Birkhauser 87, (1990), 197-218.

[HT] Harris, M. and Taylor, R.: The geometry and cohomology of some simple Shimura varieties, Princeton University Press, no. 151, Annals of Math. Studies, Princeton, New Jersey (2001).

[I1] Illusie, L.: An overview of the work of K. Fujiwara, K. Kato, and C. Nakayama on logarithmic etale cohomology, Cohomologies p-adiques et applications arithmetiques (II), Asterisque 279 (2002), 271-322.

[I2] Illusie, L.: Autour du theoreme de monodromie locale, Periodes p-adiques, Asterisque 223 (1994), 9-58.

[K] Kato, K.: Logarithmic structures of Fontaine-Illusie.

[KN] Kato, K. and Nakayama, C.: Log Betti cohomology, log etale cohomology, and $\log$ de Rham cohomology of $\log$ schemes over $\mathbb{C}$, Kodai. Math. J. 22 (1999), 161-186.

[KM] Katz, N and Mazur, B.: Arithmetic moduli of elliptic curves, Princeton University Press 1985.

[Ko1] Kottwitz, R.: Points on some Shimura varieties over finite fields, Jour. of the AMS 5 (1992), 373-444.

[Ko2] Kottwitz, R: On the $\lambda$-adic representations associated to some simple Shimura varieties, Invent. Math. 108 (1992), 653-665.

[Lan] Lan, K.-W.: Arithmetic compactifications of PEL-type Shimura varieties, Harvard thesis (2008).

[Man] Mantovan, E.: On the cohomology of certain PEL-type Shimura varieties, Duke Math. 129 (2005), 573-610. 
[Na] Nakayama, C.: Nearby cycles for log smooth families, Comp. Math. 112 (1998), 45-75.

[O] Ogus, A.: Relatively coherent log structures, Preprint.

[Ra] Ramanujan, S.: On certain arithmetical functions, Transactions of the Cambridge Philosophical Society XXII (9) (1916), 159-184. Reprinted in Ramanujan, S.: (2000), "Paper 18", Collected papers of Srinivasa Ramanujan, AMS Chelsea Publishing, Providence, RI, pp. 136-162.

[RZ] Rapoport, M. and Zink, Th.: Uber die lokale Zetafunktion von Shimuravarietaten. Monodromiefiltration und verschwindende Zyklen in ungleicher Charakteristik, Invent. Math. 68 (1982), 21-101.

[Re] Reich, R.: Notes on Beilinson's "How to glue perverse sheaves", arXiv:math.AG/1002.1686v1.

[Sa1] Saito, M.: Modules de Hodge polarisables, Publ. RIMS 24 (1988), 849995.

[Sa2] Saito, T.: Weight spectral sequences and independence of $l$, Journal of the Inst. Math. Jussieu (2003) 2(4), 583-634.

[Sar] Sarnak, P.: Notes on the generalized Ramanujan conjectures, in Arthur, J., Elwood, D. and Kottwitz, R.: Harmonic analysis, the trace formula, and Shimura varieties, Clay Math. Proc. 4 (2005), Providence, RI, Am. Math. Soc., 659-685.

[She] Shelstad, D.: L-indistinguishability for real groups, Math. Ann. 259 (1982), 385-430.

[Sh1] Shin, S. W.: Counting points on Igusa varieties, Duke Math. 146 (2009), 509-568. 
[Sh2] Shin, S.W.: Stable trace formula for Igusa varieties, J. Inst. Math. Jussieu 9 (4), (2010), 847-895.

[Sh3] Shin, S. W.: Galois representations arising from some compact Shimura varieties, preprint (2009), to appear in Ann. of Math.

[SGA4] Artin, M., Grothendieck, A. and Verdier, J.-L.: Theorie des topos et cohomologie etale des schemas, Lect. Notes Math. 305 (1972).

[SGA7] Grothendieck, A., Deligne, P. and Katz, N., with Raynaud, M. and Rim, D. S.: Groupes de monodromie en geometrie algebriques, Lect. Notes Math. 288, 340 (1972-73).

[Tad] Tadic, M.: Classification of unitary representations in irreducible representations of general linear group (non-archimeadean case), Ann. Sci. E.N.S. 19 (1986), 335-382.

[Tat] Tate, J.: Classes d'isogenie des varietes abeliennes sur un corps fini, Seminaire Bourbaki 352, Lect. Notes Math. 179 (1971).

[TY] Taylor, R. and Yoshida, T.: Compatibility of local and global Langlands correspondences, J. Amer. Math. Soc. 20 (2) (2007), 467-493.

[Y] Yoshida, T.: On non-abelian Lubin-Tate theory via vanishing cycles, to appear in Adv. Stud. in Pure Math. 